\title{
Representation Learning for Sentences and Documents
}

\section{Rui Zhao}

\section{School of Electrical \& Electronic Engineering}

A thesis submitted to the Nanyang Technological University in partial fulfillment of the requirements for the degree of Doctor of Philosophy 



\section{$\underline{\text { Statement of Originality }}$}

I hereby certify that the intellectual content of this thesis is the product of my original research work and has not been submitted for a higher degree to any other University or Institution. 



\section{Acknowledgements}

Most of all, I would like to thank Prof. Kezhi Mao for being an awesome supervisor, who offer me with the perfect balance of guidance and freedom. He made me know what a solid research work should be. Prof. Mao has generously and patiently spent much time reading, revising all of my draft papers and discussing research ideas with me, which I will never forget. Almost all methods or ideas presented in this thesis originated from those discussions with him. As an expert in machine learning, Prof. Mao let me see the nature behind lots of research problems. What is more, I learned from him not only how to conduct meaningful and high-quality research, but also the constancy to purpose in life. I believe the training I have received under his guidance will definitely benefit my future career.

I also want to thank my great advisors Prof. Ruqiang Yan in SEU and Prof. Robert X. Gao in UConn. Prof. Yan, thanks to you I tasted the beauty of research at beginning and decided to pursue my Ph.D degree. Prof. Gao, you helped me build the capability of paying attention to details. I truly admire your ability to see the nuances in everything.

I would also like to thank Dr. Wenyin Tang, Prof. Jinjiang Wang, Dr. Feng Yang, Dr. Wing Yee Sit and Dr. Xuefeng Yang. As my seniors, they have gave me a lot of valuable advices in research and life and help me analyze pros and cons of my decisions. Without their support, my graduate life may be more tough.

I want to thank these amazing members from the Internet of Things Laboratory: Dr. Zhi Zhou, Dr. Zuo Bai, Dr. Jinming Xu, Dr. Le Zhang, Dr. Zhenghua Chen, Dr. Hongmin Zhou, Dr. Kaizhou Gao, Dr. Ye Ren, Xiaoxuan Lu, Yong Zhang, Qingchang Zhu, Jie Ding, Dongzhe Wang, Xuhao Li and Qingxu Ding. The joyful time we spend together in the lab made my graduate life at NTU colorful.

Last but not least, I must also thank my dear parents. Mom and dad, you are certainly the best and I just wanted to tell you thank you and I love you so much!! 
I could not have done any of it without your love and support. I dedicate this thesis to you. 
NLP ....... Natural Language Processing

BoW ....... Bag-of-Words

RP ........ Random Projection

SRP $\ldots \ldots \ldots$ Semi-random Projection

SSRP $\ldots \ldots \ldots$ Stacked Semi-random Projection

LDA ........ Linear Discriminative Analysis

LDA $^{*} \ldots \ldots$ Latent Dirichlet Allocation

PLSA $\ldots . . .$. Probability Latent Semantic Analysis

CNN ........ Convolutional Neural Networks

LSTMs ...... Long-short Term Memory Networks

FBoW ...... Fuzzy Bag-of-Words

FBoWC ..... Fuzzy Bag-of-WordCluster

EBoW ....... Embeddings-enhanced Bag-of-Words

smSDA ...... Semantic-enhanced Marginalized Stacked Denoising Autoencoder

TopCNN .... Topic-Aware Convolutional Neural Network

TopLSTM .... Topic-Aware Long Short Term Memory Networks

PCA $\ldots . . . \ldots$. Principal Component Analysis

SVD ........ Singular Value Decomposition

LSA ......... Latent Semantic Analysis

NNLM ...... Neural Network Language Model

CBoW ...... Continuous Bag-of-Words

P.V. ........ Paragraph Vector

PV-DBOW .. Distributed Bag-of-Words Version of Paragraph Vector

PV-DM ..... Distributed Memory Version of Paragraph Vector 
RNN ....... Recurrent Neural Networks

GRU ....... Gated Recurrent Units

AdaSent ..... Self-adaptive Hierarchical Sentence Model

SDA $\ldots \ldots$..... Stacked Autoencoders

SVM ....... Support Vector Machine

mSDA $\ldots . .$. Marginalized Stacked Denoising Autoencoder

ReLU ....... Rectified Linear Unit

BTM ....... Biterm Topic Model

CV ......... Cross Validation

NBSVM .... Naive Bayes SVM

MNB ....... Multinomial Naive Bayes

RAE ....... Recursive Autoencoders

MV-RNN ... Matrix-Vector Recursive Neural Network

DSCNN ..... Dependency-sensitivity CNN

FLSC ....... Fuzzy Latent Semantic Clustering

FFC ........ Fuzzy Self-constructing Feature Clustering

WMD ....... Word Mover's Distance 


\section{Abstract}

With exponential growth of the Internet, more than one exabyte of data is created on the Internet each day. Among various kinds of data, text pose a large portion and become important in people's daily life. Text mining or natural language processing (NLP) aim to draw insights from these text data by computers, which are mainly based on machine learning. It is known that data representation determines the upper bound of the performance of machine learning algorithms. When it comes to natural language, how to define fixed-length representation for variable-length natural language units is the keystone for various tasks in text mining including text classification, document clustering and so on.

In the area of NLP, one widely adopted representation is Bag-of-Words model. However, it suffers a lot from its intrinsic extreme sparsity and is unable to capture the semantic information behind text data. Some previous research focus on feature extraction methods to derive dense and low-dimensional vectors for original BoW vectors. Recently, with the development of deep learning models, representation learning for different levels of text units has been redefined. Word embeddings attempt to encode semantic and syntactic information of words into a low-dimensional and dense vector space. For sentences and documents, multi-layer neural networks including recursive, recurrent and convolution neural networks take sequences of words as input, learn and perform compositions over word embeddings to derive distributed representation. My research in this thesis aims at learning representation for sentences and documents. The models introduced in this thesis are all developed under these two above frameworks: BoW-based models and neural compositional models. These models in this thesis are compared with various state-of-arts models among comprehensive experiments including sentiment analysis, topic categorization and so on. We also investigate the incorporation of domain knowledge into text representation learning to address one specific task: cyberbullying detection. Our proposed approaches are able to learn robust and discriminative representations of bullying messages. 
Chapter 1 is an introductory chapter that focuses on the motivation behind representation learning for text data. Chapter 2 covers the relevant techniques in this field including two major research directions: feature extraction over Bag-of-Words features and neural compositional models based on word embeddings. Chapter 3 investigates the efficiency problems in feature extraction for high-dimensional data. We propose a semi-random projection framework called SRP, which takes the merit of random feature sampling of random projection, but employs learning mechanism in the determination of the transformation matrix, with the goal of achieving a good balance between computational efficiency and classification accuracy in text categorization tasks. Chapter 4 focuses on neural compositional models for sentence embeddings. These standard works always train various forms of neural networks on top of word embeddings directly. However, the multi-sense natures of words are totally neglected by taking single prototype word embeddings as input. Hence, we explore the integration of knowledge learned from topic model into neural sentence models. With the help of Latent Dirichlet Allocation, the topic-specific sense at word level before composition and sentence-level after composition are concatenated with the general embeddings. Two different neural sentence models including Convolutional Neural Networks (CNN) and Long-short Term Memory Networks (LSTMs) are investigated to develop two corresponding methods including Topic-Aware CNN and Topic-Aware LSTMs. Comprehensive experiments over five sentence classification tasks including sentiment analysis and topic categorization are conducted. Chapter 5 relieves the BoW models assumptions that words are independent. We introduce fuzzy logic into the conventional BoW model and develop Fuzzy BoW (FBoW) and Fuzzy Bag-of-WordCluster (FBoWC) models. In $\mathrm{FBoW}$, fuzzy mapping from words in documents to basis terms is adopted. In FBoWC, fuzzy logic is adopted in not only mapping, but also selection of basis terms. To implement fuzzy logic, word embeddings are utilized to measure semantic similarities among words and construct fuzzy membership functions of basis terms in BoW space over words in the task-specific corpus. We verify the performance of our approaches through seven multi-label document categorization tasks. In Chapter 6 , we explore the application of text mining on cyberbullying detection. In this meaningful research area, the critical issue is robust and discriminative numerical representation learning of text messages. We propose two new representation learning methods to tackle this problem. One is named embeddings-enhanced bagof-words model (EBoW) and the other is named semantic-enhanced marginalized 
stacked denoising autoencoder (smSDA). Comprehensive experiments on two public cyberbullying corpora (Twitter and MySpace) are conducted, and the results show that our proposed approaches outperform other baseline text representation learning methods. Summarization of our work and future directions are given in Chapter 7 . 



\section{Contents}

Acknowledgements $\quad$ i

Acronym List

$\begin{array}{lll}\text { Abstract } & \text { V }\end{array}$

List of Figures xiii

1 Introduction 1

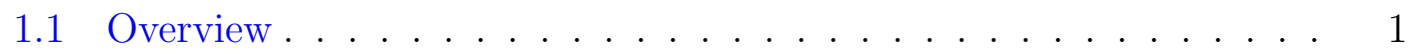

1.2 Contributions and Outline of this Thesis . . . . . . . . . 5

1.3 Author's Publications ................. 8

2 Literature Review of Text Representation Learning 11

2.1 BoW-based Models . . . . . . . . . . . . . . . . . . . 12

2.1.1 Principal Component Analysis . . . . . . . . . . . . . 13

2.1.2 Random Projection . . . . . . . . . . . . . . . . 14

2.1.3 Analysis of BoW-based Models . . . . . . . . . . . . . . 15

2.2 Word Embeddings . . . . . . . . . . . . . . . . . . . . . . . . . . . . . . 15

2.2.1 Word2Vec . . . . . . . . . . . . . . . . . . . . . . . . . . . . 16

2.2.2 Paragraph Vector . . . . . . . . . . . . . . . . . . 18

2.3 Semantic Compositional Models . . . . . . . . . . . . . . . . . 18

2.3.1 Shallow Compositional Models . . . . . . . . . . . . 20

2.3.2 Neural Compositional Model . . . . . . . . . . . . . . 20

2.3.3 Analysis of Neural Compositional Models . . . . . . . . . . . 28

3 Semi-Random Projection for Document Representation 31

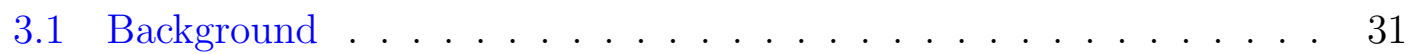

3.1.1 Motivations . . . . . . . . . . . . . . 31

3.2 Semi-Random Projection for Dimensionality Reduction . . . . . . . 33

3.2.1 Random Feature Subset Selection and Transformation Vector Learning . . . . . . . . . . . . . . . . . . . 35

3.2 .2 Algorithm Details . . . . . . . . . . . . . . . . . . . . . . . . . . . . . 36

3.3 Stacked Semi-Random Projection . . . . . . . . . . . . . 37 
3.4 Discussions . . . . . . . . . . . . . . . . . . . . . . 39

3.4.1 Random Settings . . . . . . . . . . . . . . . . . . . . . . . . . 39

3.4.2 Computational Complexity . . . . . . . . . . . . . . . 40

3.4.3 Sparsity of Transformation Matrix . . . . . . . . . . . . . . 40

3.4.4 Stacked Structures . . . . . . . . . . . . . . . . . . 41

3.4.5 Merits of Semi-Random Projection (SRP) . . . . . . . . . 41

3.5 Experiments . . . . . . . . . . . . . . . . . 42

3.5.1 Datasets . . . . . . . . . . . . . . . 42

3.5.2 Evaluation Metrics . . . . . . . . . . . . . . . . . . . . 43

3.5.3 Compared Approaches . . . . . . . . . . . . . . . 43

3.5.4 Performance Comparison on all Datasets . . . . . . . . . . . 46

3.5.5 Analysis of Number of Layers . . . . . . . . . . . . . . . . . . 48

3.6 Conclusion . . . . . . . . . . . . . . . . . . . . . . . . . . . . . . . 49

4 Topic-Aware Deep Compositional Models for Sentence Represen$\begin{array}{ll}\text { tation } & 51\end{array}$

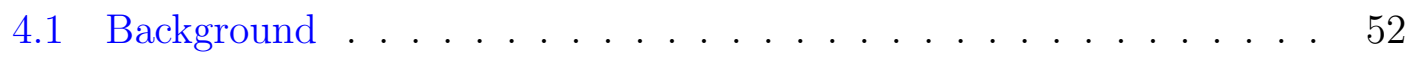

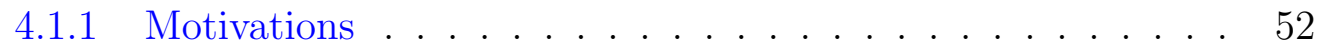

4.1 .2 Topic Models . . . . . . . . . . . . . . . . 53

4.1.3 Multi-sense Word Embeddings for Semantic Composition . . 54

4.2 Topic-Aware Deep Compositional Models . . . . . . . . . . . . . . . 55

4.2.1 Topic-Aware CNN . . . . . . . . . . . . . . . . . . 57

4.2 .2 Topic-Aware LSTM . . . . . . . . . . . . . . . . . 61

4.2.3 Embeddings Learning for Topics . . . . . . . . . . . . . . 61

4.2 .4 Complexity Analysis . . . . . . . . . . . . . . . 64

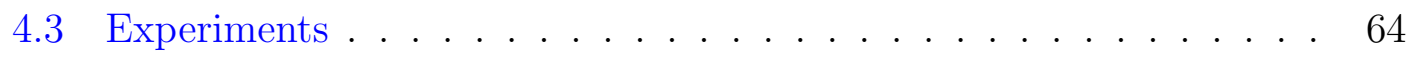

4.3.1 Experimental Settings . . . . . . . . . . . . 64

4.3.2 Specifications for TopCNN . . . . . . . . . . . . . 67

4.3.3 Experimental Results . . . . . . . . . . . . . 68

4.3.4 Sensitivity to the Number of Topics . . . . . . . . . . . . 72

4.3.5 Discussion on Topic Embeddings Learning . . . . . . . . . . 72

4.4 Conclusion . . . . . . . . . . . . . . . . . 75

5 Fuzzy Bag-of-Words Model for Document Representation $\quad 79$

5.1 Background ....................... 79

5.1 .1 Motivations .................... 80

5.1.2 Fuzzy System and Its Applications in Text Mining . . . . . . 81

5.2 Fuzzy Bag-of-Words ... . . . . . . . . . . . . . . . 82

5.2 .1 Word Embeddings . . . . . . . . . . . . . . . . . 83

5.2.2 Fuzzy Bag-of-Words Model . . . . . . . . . . . . . . . 84

5.2.3 Fuzzy Bag-of-WordClusters Model . . . . . . . . . . . . 86

5.2.4 Relationships with Previous Text Representation Methods . 88

5.3 Experiments . . . . . . . . . . . . . . . . . . . . . . 91

5.3.1 Descriptions of Datasets . . . . . . . . . . . . 91 
5.3.2 Experimental Setup . . . . . . . . . . . . . . . 92

5.3.3 Experimental Results on Document Categorization Tasks . . 94

5.3.4 Experimental Analysis of Mapping Bounds . . . . . . . . . . 96

5.4 Conclusion . . . . . . . . . . . . . . . . . . 97

6 Representation Learning incorporating domain knowledge for Cy$\begin{array}{ll}\text { berbullying Detection } & 101\end{array}$

6.1 Background . . . . . . . . . . . . . . . . . . . . . 101

6.1.1 Motivations . . . . . . . . . . . . . . . . . 101

6.1.2 Related Works . . . . . . . . . . . . . . . . 103

6.2 Embeddings-enhanced Bag-of-Words Model . . . . . . . . . . . . . 104

6.2.1 EBoW: Embeddings-enhanced Bag-of-Words Model . . . . . 104

6.2.2 Experiments . . . . . . . . . . . . . . 107

6.3 Semantic-Enhanced Marginalized Stacked Denoising Auto-encoder for Cyberbullying Detection . . . . . . . . . . . . . . . . . . 112

6.3.1 mSDA: Marginalized Stacked Denoising Auto-encoder . . . . 114

6.3.2 smSDA: Semantic-Enhanced Marginalized Stacked Denoising Auto-encoder . . . . . . . . . . . . 116

6.3.3 smSDA for Cyberbullying Detection . . . . . . . . . . . 121

6.3.4 Merits of smSDA . . . . . . . . . . . . . . 122

6.3.5 Experiments . . . . . . . . . . . . . . 123

6.4 Conclusions . . . . . . . . . . . . . . . . . . . . 133

7 Conclusions $\quad \mathbf{1 3 5}$

7.1 Summary . . . . . . . . . . . . . . . . . . . 135

7.2 Future Directions . . . . . . . . . . . . . . . . . . . . 137

$\begin{array}{ll}\text { Bibliography } & 141\end{array}$ 



\section{List of Figures}

1.1 A toy example on the BoW model's failures to capture semantics.

1.2 Illustration of Neural Compositional Models built on top of Word Embeddings. . . . . . . . . . . . . . . . . . 4

2.1 Comparison between conventional one-hot word vector and word embeddings. . . . . . . . . . . . . . . . . . . 17

2.2 The Framework for Neural Network Language Model. . . . . . . . . 17

2.3 The Frameworks for two word2vec models: CBoW and Skip-gram. . 18

2.4 Illustrations of Distributed Bag of Words version of Paragraph Vec-

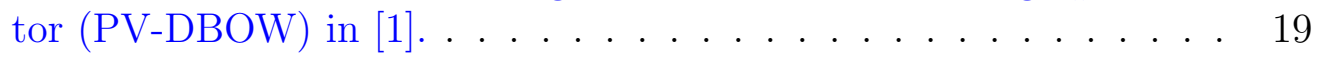

2.5 Illustrations of Distributed Memory version of Paragraph Vector $(\mathrm{PV}-\mathrm{DM})$ in $[1] \ldots \ldots \ldots \ldots$

2.6 Illustrations of CNN for sentence representation learning. . . . . . . 23

2.7 Illustrations of Recurrent Neural Networks for sentence representation learning. . . . . . . . . . . . . . . . . . 26

2.8 Illustrations of normal RNN, stacked RNN and bidirectional RNN. . 26

2.9 Illustrations of Recursive Neural Networks for sentence representation learning. . . . . . . . . . . . . . . . . . . 27

2.10 Illustrations of Self-adaptive Hierarchical Sentence Model for sentence representation learning in $[2] \ldots \ldots$. . . . . . . . . . . . . 29

3.1 Illustrations of the possible ineffectiveness of Random Projection . . 33

3.2 Unified graph explanation for Random Projection and Semi-Random Projection .......................... 37

3.3 Architecture of Stacked SRP with $l$ layers. . . . . . . . . . . . . . . 39

3.4 Dimensionality on the seven datasets (the first six ones belong to 20Newsgroup and the final one belongs to farm ads) at the output of different layers in SSRP . . . . . . . . . . . . . . . . 49

3.5 Classification accuracies (\%) on the seven datasets at the output of different layers in SSRP . . . . . . . . . . . . . . . 50

4.1 Illustration of Semantic Composition Models built on top of MultiSense Embedding. . . . . . . . . . . . . . . . . 56

4.2 The Framework of our TopCNN models for sentence representation learning. . . . . . . . . . . . . . . 56 
4.3 The Framework of our TopLSTMs for sentence representation learning. . . . . . . . . . . . . . . . . .

4.4 Two neural network architectures (Skip-gram based) for topic embeddings learning: (a) Topical Neural Model; (b) Joint Neural Model.

4.5 Performances gains achieved by our proposed TopCNN and TopLSTMs models compared to CNN and LSTM, respectively . . . . . . . . . 71

4.6 Performances of our proposed three models for different number of topics on MR dataset. The numbers of topics are ranged from 0 to 300 with a step size of $50 . \ldots \ldots$. . . . . . . . . . .

4.7 Performances of our proposed three models for different number of topics on Subj dataset. The numbers of topics are ranged from 0 to 300 with a step size of 50 .

4.8 Performances of our proposed three models for different number of topics on CR dataset. The numbers of topics are ranged from 0 to 300 with a step size of 50 .

4.9 Performances of our proposed three models for different number of topics on MPQA dataset. The numbers of topics are ranged from 0 to 300 with a step size of $50 \ldots \ldots \ldots \ldots$

4.10 Performances of our proposed three models for different number of topics on TREC dataset. The numbers of topics are ranged from 0 to 300 with a step size of $50 \ldots \ldots \ldots$. . . . . . . .

4.11 Effects of different topics embeddings learning schemes on the whole five datasets.

5.1 Illustration and Comparison between Bag-of-Words and our proposed Fuzzy Bag-of-Words models based on a toy example : (a) Bag-of-Words; (b) Fuzzy Bag-of-Words.

5.2 Illustrations of membership functions behind Bag-of-Words and our proposed Fuzzy Bag-of-Words models : (a) Bag-of-Words; (b) Fuzzy Bag-of-Words. . . . . . . . . . . . . . .

5.3 Illustrations of mean, maximum and minimum similarity measures between clusters and words. It is noted that a high similarity measure denotes a small distance shown in the Figure. . . . . . . . . .

5.4 Performance of BoW and $\mathrm{FBoWC}_{\text {mean }}$ for different dimensionality $l$ on 20 newsgroups. . . . . . . . . . . . . . . . . . . . . 97

5.5 Performance of $\mathrm{FBoW}, \mathrm{FBoWC}_{\text {mean }}$ and $\mathrm{FBoWC}_{\max }$ for different

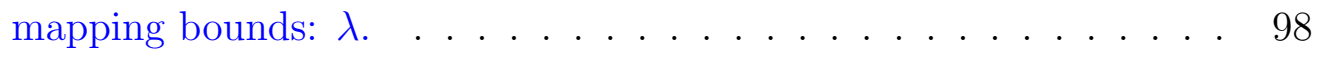

6.1 Illustration of final representation in our proposed EBoW model. . 107

6.2 Two dimensional visualization of our used word embeddings via PCA. Displayed terms include both bullying ones and normal ones. It shows that similar words are nearby vectors. . . . . . . . . . . 108

6.3 Some Examples from Twitter Datasets. Three of them are nonbullying traces. And the other three are bullying traces. . . . . . . 109 
6.4 Performance of our proposed EBoW model for different $h$. Different colors indicate different measures. . . . . . . . . . . . . . . . . 112

6.5 Illustration of Motivations behind smSDA. In Figure 1(a), the cross symbol denotes that its corresponding feature is corrupted, i.e., turned off. . . . . . . . . . . . . . . . . . . . . . . 120

6.6 Some Examples from MySpace Datasets. Two Conversions are Displayed and each one includes a normal post $(P)$ and a bullying post $\left(B \_P\right) \ldots \ldots \ldots \ldots \ldots \ldots \ldots$

6.7 Word Cloud Visualization of the List of Words with Negative Affective.127

6.8 Word Cloud Visualization of the Bullying Features in Twitter Datasets. 128

6.9 Word Cloud Visualization of the Bullying Features in MySpace Datasets. 129

6.10 Classification Accuracies and F1 Scores of All Compared Methods on Twitter Datasets. . . . . . . . . . . . . . . . . 131

6.11 Classification Accuracies and F1 Scores of All Compared Methods on MySpace Datasets. . . . . . . . . . . . . . . . . . . 132 



\section{Chapter 1}

\section{Introduction}

\subsection{Overview}

Big Data is a hot topic in the world. Sensors used to measure human activity, tweets or other social media posts, digital photos and videos, supply chain tools, cell phone global positioning system (GPS) signal and customer relationship management (CRM) information - various kinds of data are flooding into our world [3, 4]. Every day, 2.5 quintillion bytes of data are created. Via analyzing such enormous volumes of data, better decisions can be made. As an important portion of all the data, text can be regarded the most natural form of storing information. For example, huge amount of text data including blogs and posts generated by web pages and social medias demonstrates lots of information including people's behavior and thought. Therefore, it is meaningful to process text data and extract knowledge from them automatically and intelligently, which are known as text mining and natural language processing.

However, different from structure data that reside in a fixed field within a record or file, text data belong to unstructured data and is opposite to machine-language. Therefore, in text mining and natural language processing, the important and core step is transforming these unstructured text data into numerical vectors, which is defined as text representation learning. It is well known that effective machine learning algorithms should be supported by structured numerical representation of data and the good representation determines the upper-bound of the performance of machine learning algorithms [5]. And representation learning of text is 
the keystone for various text mining tasks including text classification, document clustering and so on [6-9].

One commonly adopted and effective approach for document representation is the Bag-of-Words (BoW) model [10-13]. BoW model assigns a vector to a document as $\boldsymbol{d}=\left(x_{1}, x_{2}, \ldots, x_{l}\right)$, where $x_{i}$ denotes the normalized number of occurrence of the $i$-th basis term and $l$ is the size of the collection of basis terms. BoW is a simple and effective method to map a document into a fixed-length vector. However, BoW model has several shortcomings:

* High Dimensionality: The dimensionality of learned BoW vectors is the size of vocabulary. And the vocabulary size is easily over 10,000 for a middle sized corpus. High dimensionality makes the distance metrics become less meaningful, and this in turn results in deteriorated performances for many distance-based data mining approaches. High dimensionality also poses problems for computations.

* Extreme Sparse: Word usage in each document only cover a small portion of the whole vocabulary. As a result, the elements of the learned BoW vector have mostly zero values.

* Abandon of Word Order: BoW model only counts the occurrence of words and does not consider the sequential nature of words. Due to the lack of word order information, it is hard to capture complex semantic meaning.

Therefore, the representations learned by BoW may not effectively capture semantics of text since semantical similar documents with different word usages are mapped to very different vectors under BoW. For example, as shown in Figure 1.1, there exists a strong semantic correlation between two sentences $d_{1}$ " $a$ dog is above the bench" and $d_{2}$ "the huskies is on the table". If the BoW employs the following basis terms: \{ "above", "on", "table", " $\operatorname{dog} "\}$, the two sentences $d_{1}$ and $d_{2}$ will be projected to $\boldsymbol{d}_{1}=(1,0,0,1)$ and $\boldsymbol{d}_{2}=(0,1,1,0)$ respectively, as shown in Figure 1.1. Thus, the cosine similarity measure between two learned vectors $d_{1}$ and $d_{2}$ is 0 , which is contrary to the true semantic similarity between the two sentences. To overcome the above issues especially sparsity and high dimensionality, various dimensionality reduction techniques have been applied on BoW features. Then, 


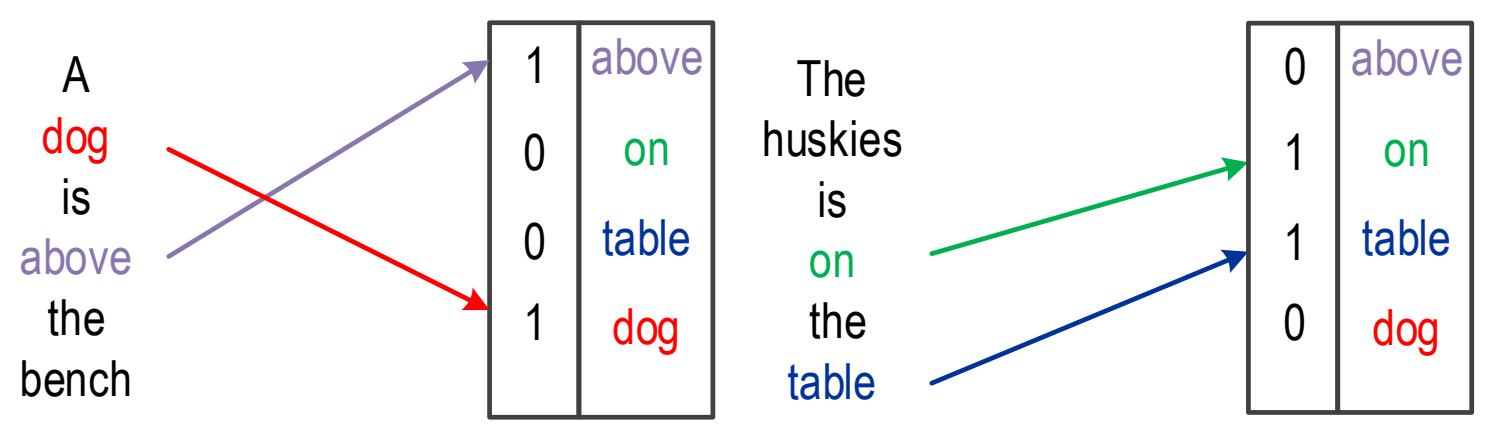

BoW Model

Figure 1.1: A toy example on the BoW model's failures to capture semantics.

these representations are fed into the following machine learning models. In my thesis, this kind of models for text representation is named BoW-based methods.

It is clear that BoW-based methods ignore word order information in sentences and documents. With the development of deep learning methods in the last few years, representation learning for different levels of text units has been redefined [1416]. In deep learning models for NLP, the compositional models are built on word embeddings as shown in Figure 1.2. In this framework, deep compositional models are built on top of word embeddings and regard sentences as sequences of words to capture its sequential information. Word embeddings, in contrast to one-hot word representation which is a extreme sparse 1 -of- $V$ vector ( $V$ is the vocabulary size), encode semantic and syntactic information of words into a low-dimensional and dense representation through neural language models trained on a large-scale text corpus without annotations [17-19]. Another important ingredient of deep learning models is the applied compositional models. Before deep learning models become popular, the commonly used models are shallow models based on fixed algebraic operations including addition, tensor production and so on [20-23]. Neural models mainly adopt multi-layer neural networks including recursive [24, 25], recurrent [26, 27] and convolutional [28-30] neural networks to learn and perform compositions over word embeddings. These models are called neural compositional models in the following. It seems that neural compositional models may be perfect text representation models that can derive dense and low-dimensional representations via considering word order information. However, there are several drawbacks behind these models. Firstly, due to the complexity of neural compositional models, a large annotated corpus is required. Secondly, these applied neural models are only capable of short sequence modeling. Therefore, neural compositional models may 


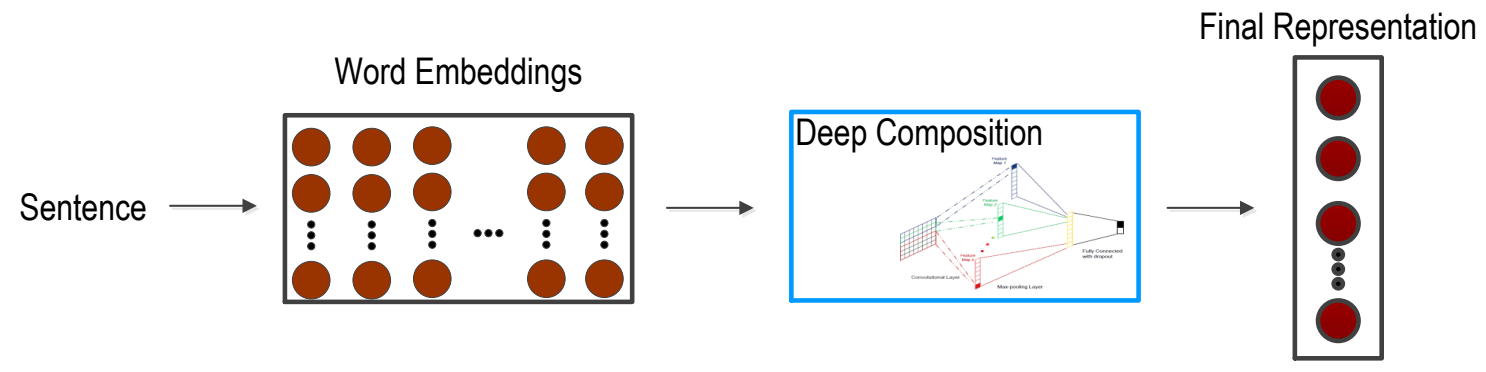

Figure 1.2: Illustration of Neural Compositional Models built on top of Word Embeddings.

not model documents or even long sentences effectively. At last, most of these neural compositional models neglect the multisense nature of words.

In this thesis, the proposed models are all developed in these two above fields including BoW-based methods and neural compositional models to address their intrinsic shortcomings. These proposed models are aimed at learning distributed (dense and low-dimensional) representations for documents or sentences. To verify the performances of learned representations, various text classification tasks including topic classifciation and sentiment analysis have been introduced. It should be note that our focus is not text classifcaiton, which are different from recent text classifciation models such as fast-text [31]. The main research objectives in this thesis are given as follows:

1 reduce the high dimensionality of BoW features efficiently and effectively;

2 evaluate the effects of multisense words on sentence/document representation learning and incorporate it into deep compositional models;

3 overcome the exact word-matching in BoW model by proposing fuzzy mapping based on word embeddings;

4 develop effective cyberbullying detection systems based on text classification models, in which domain knowledge is incorporated into text representation learning. 


\subsection{Contributions and Outline of this Thesis}

To draw insights from unstructured text data which is the goal of text mining and natural language processing, the important and core step is transforming these unstructured text data into numerical vectors. This problem is referred to as text representation learning. Representation learning of text is the keystone for various text mining tasks including text classification, document clustering and so on. In this thesis, we investigated various text representation learning models in the above two research directions: BoW-based methods and neural compositional models. And topic categorization, sentiment analysis and other general text classification tasks are introduced to evaluate the quality of learned representations. Besides that, one specific problem: cyberbullying detection is considered. We explored the incorporation of domain knowledge into text representation models to detect bullying messages effectively.

In Chapter 2, various models in these two major research directions for text representation learning including BoW-based methods and neural compositional models are reviewed, respectively. In Chapter 3, Chapter 4 and Chapter 5, three general text representation learning frameworks are presented, respectively. In Chapter 6, the applications of text representation learning models on one practical and meaningful area: cyberbullying detection are investigated. Finally, brief conclusion and deep discussion of our work are given with an introduction of potential future works in Chapter 7.

\section{Chapter 3: semi-random projection for text representation}

The model in this section belongs to BoW-based methods, which investigates the efficiency problems in feature extraction for high-dimensional BoW features. Random projection (RP) is a popular technique for dimensionality reduction because of its high computational efficiency. However, RP may not yield highly discriminative low-dimensional space to produce best pattern classification performance since the random transformation matrix of RP is independent of data. Therefore, a semirandom projection (SRP) framework has been proposed, which takes the merit of random feature sampling of RP, but employs learning mechanism in the determination of the transformation matrix. One advantage of SRP is that it achieves a good balance between computational complexity and classification accuracy. Another advantage of SRP is that multiple SRP modules can be stacked to form a 
deep learning architecture for compact and robust feature learning, which is named stacked semi-random projection (SSRP). Experiments on two real-world text corpus, i.e., 20 Newsgroups and Farms Ads., verify the effectiveness and efficiency of the proposed SRP. Although we focus on text representation learning in this chapter, the proposed SRP techniques and related analysis are not restricted to the domain of text representation problems but can be generalized to data representation in high-dimensional space. Therefore, this chapter is written in the context of high-dimensional data, which is based on our paper "Semi-random projection for dimensionality reduction and extreme learning machine in high-dimensional space" $[32]$.

Chapter 4: topic-aware deep compositional models for sentence representation The second exploration in my thesis focus on neural compositional models. In recent years, neural compositional models have emerged as a popular technique for representation learning of sentences in computational linguistic and natural language processing. These models normally train various forms of neural networks on top of pre-trained word embeddings using a task-specific corpus. However, most of these works neglect the multi-sense nature of words in the pre-trained word embeddings. In this chapter, we introduce topic models to enrich the word embeddings for multi-senses of words. The integration of the topic model with various semantic compositional processes leads to Topic-Aware Convolutional Neural Network (TopCNN) and Topic-Aware Long Short Term Memory Networks (TopLSTMs). Different from previous multi-sense word embeddings models that assign multiple independent and sense-specific embeddings to each word, our proposed models are lightweight and flexible frameworks that regard word sense as the composition of two parts: a general sense derived from a large corpus and a topic-specific sense derived from a task-specific corpus. With the help of topic models, we can integrate the topic-specific sense at word-level before composition and sentence-level after composition. Comprehensive experiments on five public sentence classification datasets are conducted, and the results show that our proposed Topic-Aware deep compositional models produce competitive or better performance than other text representation learning methods. This chapter is based on our paper "TopicAware Deep Compositional Models for Sentence Classification" [33].

Chapter 5: fuzzy bag-of-words for document representation

The proposed work in this chapter incorporated fuzzy mapping into the original 
BoW model. In a BoW-based vector representation of a document, each element denotes the normalized number of occurrence of a basis term in the document. To count the number of occurrence of a basis term, BoW conducts exact word matching, which can be regarded as a hard mapping from words to the basis term. BoW representation suffers from its intrinsic extreme sparsity, high dimensionality, and inability to capture high-level semantic meanings behind text data. To address the above issues, we propose a new document representation method named Fuzzy Bag-of-Words (FBoW) in this chapter. FBoW adopts a fuzzy mapping based on semantic correlation among words quantified by cosine similarity measures between word embeddings. Since word semantic matching instead of exact word string matching is used, the FBoW could encode more semantics into the numerical representation. In addition, we propose to use word clusters instead of individual words as basis terms and develop Fuzzy Bag-of-WordClusters (FBoWC) models. Three variants under the framework of FBoWC are proposed based on three different similarity measures between word clusters and words, which are named as $\mathrm{FBoWC}_{\text {mean }}, \mathrm{FBoWC}_{\max }$ and $\mathrm{FBoWC}_{\text {min }}$, respectively. Document representations learned by the proposed FBoW and FBoWC are dense and able to encode highlevel semantics. In addition, the relationship between this work and the two major research fields: BoW-based methods and neural compositional models are discussed. The task of document categorization is used to evaluate the performances of learned representations by the proposed FBoW and FBoWC methods. The results on seven real word document classification datasets in comparison with six document representation learning methods have shown that our methods FBoW and FBoWC achieve the highest classification accuracies. This chapter is based on our paper "Fuzzy Bag-of-words Models For Document Representation" [34].

Chapter 6: Representation Learning incorporating domain knowledge for cyberbullying detection

Different from previous introduced models, the work in this chapter focus on one specified application: cyberbullying detection. Machine learning techniques make automatic detection of bullying messages in social media possible, and this could contribute to a healthy and safe social media environment. In this meaningful research area, one critical issue is robust and discriminative numerical representation learning of text messages. In this chapter, we propose two novel representation learning methods to tackle this problem via incorporating domain knowledge. This 
first one is named Embedding-enhanced Bag-of-Words (EBoW). EBoW concatenates BoW features, latent semantic features and bullying features together, in which bullying features are derived based on domain knowledge and word vectors. The other one is semantic-enhanced marginalized denoising auto-encoder ( $\mathrm{smSDA}$ ), which is developed via semantic extension of the popular deep learning model stacked denoising autoencoder. The semantic extension consists of semantic dropout noise and sparsity constraints, where the semantic dropout noise is designed based on domain knowledge and the word embedding technique. This chapter is based on our papers "Cyberbullying detection based on semantic-enhanced marginalized denoising auto-encoder" and "Automatic detection of cyberbullying on social networks based on bullying features" [35, 36].

\subsection{Author's Publications}

In this section, relevant papers published in my Ph.D study are provided.

\section{Journal Articles}

- Rui Zhao, Kezhi Mao: Fuzzy Bag-of-Words Model for Document Representation. IEEE Transactions on Fuzzy System, 2017. (Accepted)

- Rui Zhao, Kezhi Mao: Topic-Aware Deep Compositional Models for Sentence Classification. IEEE/ACM Transactions on Audio, Speech, and Language Processing, 25 (2):248260, Feb 2017.

- Rui Zhao, Kezhi Mao: Cyberbullying Detection based on Semantic-Enhanced Marginalized Denosing Auto-Encoder. IEEE Transactions on Affective Computing, 2016. (Accepted).

- Rui Zhao, Kezhi Mao: Semi-Random Projection for Dimensionality Reduction and Extreme Learning Machine in High-dimensional Space. IEEE Computational Intelligence Magazine, 10.3 (2015): 30-41.

\section{Conference Proceedings}


- Rui Zhao, Anna Zhou and Kezhi Mao: Automatic Detection of Cyberbullying on Social Networks based on Bullying Features. International Conference on Distributed Computing and Networking (ICDCN 2016)

- Rui Zhao, Kezhi Mao: Supervised Adaptive-transfer PLSA for Cross-Domain Text Classification. Data Mining Workshops (ICDMW), 2014 IEEE 14th International Conference on. IEEE, 2014. 



\section{Chapter 2}

\section{Literature Review of Text Representation Learning}

In this chapter, we try to review and analyze classical and popular text representation learning methods in an systematic way. The potential concerns behind these methods will be highlighted, which motivate us to propose novel models to address them. We first introduce BoW model and feature extraction methods based on BoW features, which is called BoW-based models in section 2.1. In section 2.2, word embeddings are introduced, which make the application of deep learning on NLP possible. Our proposed models in Chapter 4, Chapter 5 and Chapter 6 all adopt word embeddings. In addition, paragraph vectors models are reviewed. In section 2.3, we then briefly review the framework of neural composition models based on word embeddings and introduce three basic models including convolutional neural network, recurrent neural network and recursive neural network and one recent model named self-adaptive hierarchical sentence model. Classical models (BoW-based models) and recent popular models (neural compositional models) all have their own advantages and disadvantages. Therefore, analysis of these previous models including BoW-based models and neural compositional models are provided in subsections 2.1.3 and 2.3.3, respectively. We are going to provide some insights into these models. And in turn, these insights motivate the development of our works. 


\subsection{BoW-based Models}

The bag-of-words model is the classical vector representation for text due to its efficiency and surprising effectiveness [37]. In this model, text data is represented as the bag of its words without considering word orders. Words would be represented by vectors with the dimension as the vocabulary size. Each word vector contains zeros in all dimensions except for the one in the position corresponding to the word. Sentences or documents would be represented by vectors which contain the term weighting. In the task of document classification, there are various term weighting schemes, in which the most popular one is the tf-idf weighting [38]. When the text data are represented as fixed-length vectors by the BoW models, these numerical representation can be fed as input into traditional machine learning algorithms, including support vector machine and logistic regression.

BoW can represent documents by fixed-length vectors indicating the occurrence of word in the documents without preserving word order. It is obvious that the disadvantages of this BoW model can be concluded as follows: its high dimensionality, sparsity and inability to solve synonym and polysemy. Therefore, dimensionality reduction has been proposed to overcome these shortcomings of this BoW. The key idea behind these dimensionality reduction techniques is the extraction of hidden/semantic features from the the original feature space.

Dimensionality reduction techniques seek to reduce the rank of vectors. Through dimensionality reduction, BoW vectors can be transformed into dense and lowdimensional ones, which can boost the efficiency of subsequent models making use of such representations. For text categorization, the standard approach is after the dimensions of BoW vectors for documents are reduced, a standard classifier such as SVM, logistic regression and nearest neighbor classifier are used to assign labels to test documents based on training corpus with labels.

In the following, we will review two popular techniques for dimensionality reduction in text mining principal component analysis (PCA) and random projection (RP), respectively.

Assuming that the dataset is denoted by a matrix $\mathbf{X} \in \mathbb{R}^{d \times N}$, where $d$ and $N$ are the dimension and sample number of data, and the dataset has an intrinsic dimensionality $r(r<d)$. Linear dimensionality reduction can be formulated as a 
mapping from the original space to a low dimensional space:

$$
\mathbf{H}=\mathbf{W}^{T} \mathbf{X}
$$

where ${ }^{T}$ denotes the matrix transpose, $\mathbf{H} \in \mathbb{R}^{r \times N}$ denotes the new data representation, and $\mathbf{W} \in \mathbb{R}^{d \times r}$ is the linear transformation matrix. The key issue in linear dimensionality reduction is to find the transformation matrix $\mathbf{W}$.

\subsubsection{Principal Component Analysis}

Principal component analysis (PCA) is a classical feature extraction and dimensionality reduction technique. As an unsupervised method, PCA attempts to find a latent space that maximizes preservation of variance of the original feature space so as to minimize information loss caused by dimensionality reduction [39]. PCA is widely used in applications that suffer from high dimensionality of data. The optimization problem for PCA can be summarized as following:

$$
\mathbf{W}^{*}=\underset{\mathbf{W} \in \mathbb{R}^{d \times r}}{\operatorname{argmax}} \operatorname{Tr}\left(\frac{\mathbf{W}^{T} \mathbf{L W}}{\mathbf{W}^{T} \mathbf{B W}}\right)
$$

where $\operatorname{Tr}$ denotes the trace of a matrix. Intuitively, $\mathbf{L}$ represents the quantity that we would like to enhance and $\mathbf{B}$ denotes the quantity that we would like to suppress. Different settings for $\mathbf{L}$ and $\mathbf{B}$ lead to different Dimensionality Reduction approaches. For PCA, these two matrices $\mathbf{L}$ and $\mathbf{B}$ are defined as follows:

$$
P C A:\left\{\begin{array}{l}
\mathbf{L}=\frac{1}{N} \sum_{i=1}^{N}\left(\mathbf{x}^{i}-\overline{\mathbf{x}}\right)\left(\mathbf{x}^{i}-\overline{\mathbf{x}}\right)^{T}, \\
\mathbf{B}=\mathbf{I}_{\mathbf{d}}
\end{array}\right.
$$

where $\overline{\mathbf{x}}$ is the mean vector of all samples, $\mathbf{I}_{\mathbf{d}}$ denotes a d-dimensional identity matrix with and $\mathbf{L}$ is actually the covariance matrix.

PCA is a popular technique for dimensionality reduction [39]. In PCA, singular value decomposition (SVD) is performed on the data matrix to derive a latent space spanned by dominant eigenvectors corresponding to large eigenvalues [40]. It has been shown that the retained variance of data projected on the latent space is maximal. Each new feature is a linear combination of all original features. PCA serves as a basic model for many algorithms including kernel PCA [41], probabilistic 
PCA [42] and supervised probabilistic PCA [43]. In text mining, PCA applied on the document-term matrix, also known as Latent Semantic Analysis, is often adopted to efficiently represent documents [44].

Although PCA has been applied in many applications, it has limitations. For example, PCA only seeks to map the data onto the directions with maximum variance, and the resulted low dimensional space may not have sufficient discriminative power, which in turn deteriorate the performance of pattern classification. For very high-dimensional data, saying the dimension is over 10000 in text mining applications, the computational cost is almost prohibitive, considering the cost for solving Eq. (2.2) is $\mathcal{O}\left(d^{3}\right)$. The higher the dimension of data, the heavier the computation burden for these dimensionality reduction approaches.

\subsubsection{Random Projection}

In [45] and [46], random projection has been proposed as a dimensionality reduction method to address the computational complexity issue. In RP, the transform matrix $\mathbf{W}$ is generated randomly. The experimental results in [45, 46] have shown that RP can achieve a significant speedup in computation time with little distortion of pairwise information of data. However, as pointed out in [45], if the distances or similarities are not useful in the original space, the performance of RP should be suspected. It was proven in [47] that for any pairwise points in the high dimensional space, the similarity measures may become meaningless. This indicates that for pattern classification tasks, the low-dimensional space generated by random projection may not derive a useful feature space. In other view, without data-based parameter tuning in transformation vectors, RP may not capture the task-related information underlying the original data.

As suggested in [46], one of the typical random assignments for the transformation matrix $\mathbf{W}$ is based on an i.i.d sampling described by:

$$
w_{i, j}=\sqrt{c} \begin{cases}1 & \text { with prob. } \frac{1}{2 c}, \\ 0 & \text { with prob. } 1-\frac{1}{c}, \\ -1 & \text { with prob. } \frac{1}{2 c} .\end{cases}
$$

where $c$ is set to $\sqrt{d}$. 


\subsubsection{Analysis of BoW-based Models}

In a BoW-based vector representation of a document, each element denotes the normalized number of occurrence of a basis term in the document. To count the number of occurrence of a basis term, BoW conducts exact word matching, which can be regarded as a hard mapping from words to the basis term. BoW representation suffers from its intrinsic extreme sparsity, high dimensionality, and inability to capture high-level semantic meanings behind text data. Feature extraction methods are able to alleviate these concerns. This solution leads to our proposed semi-random projection (SRP) framework, which is presented in Chapter 3. We also explore another solution named fuzzy BoW model that adopts a fuzzy mapping based on semantic correlation among words quantified by cosine similarity measures between word embeddings in Chapter 5 .

\subsection{Word Embeddings}

In [48], word embeddings were firstly proposed that words are represented by continuous and dense vectors. Different from the one-hot representation in BoW models without semantic meaning, each dimension in word embeddings represents latent meanings including semantic and syntactic ones, as shown in Figure 2.1. The core idea behind word embeddings is to assign such a dense and low-dimensional vector representation to each word that semantically similar words are close to each other in the vector space. The merit of word embeddings is that the semantic similarity between two words can be conveniently evaluated based on the cosine similarity measure between corresponding vector representations of the two words. Therefore, the distance metrics between word embedding can be introduced directly to measure the semantic similarity between the corresponding words. One recent study pointed out that neural word embeddings is trained to preserve the patterns inherent in the wordcontext co-occurrence matrix, which is similar to explicit word embeddings [49]. In addition, their work also stated that both sparse and dense representations are capable of revealing analogies on word-level..

Neural language models are usually applied to induce word embeddings, where neural networks structures are introduced to predict word occurrence based on 
context information. This kind of work was firstly proposed in [50] named neural network language model (NNLM), whose framework is shown in Figure 2.2. The aim of these models is to learn language model and word embeddings jointly. NNLM aims at inferring the probability distribution over sequences of words that is simplified as the conditional probability: $\operatorname{Pr}\left(w_{i} \mid w_{i-(n-1)}, w_{i-(n-2)}, \ldots, w_{i-1}\right)$. The optimization of the model can be defined to maximize the conditional probability for each word sequence $w_{i-(n-1)}, w_{i-(n-2)}, \ldots, w_{i-1}, w_{i}$ over the corpus. Since word embeddings are adopted as input layer of the proposed NNLM model, language model and word embeddings can be trained jointly. However, these models have always been criticised for its high computing complexity scaling to the size of the vocabulary, since the size of the vocabulary determines the output layer's size. To overcome this shortcoming, many approaches have been proposed [19, 51-53]. The most popular and famous model may be word2vec, which is a two-layer neural network language model $[19,53]$. Its details will be introduced in the following section.

\subsubsection{Word2Vec}

The word2vec framework contains two separate models including continuous bag of words $(\mathrm{CBoW})$ and skip-gram with two reverse training goals. As shown in Figure 2.3, CBoW tries to predict a word given the surrounding words, while Skipgram tries to predict a window of words given a single word. The maximization objectives of these two above word2vec structures are defined as follows:

$$
\begin{gathered}
L(s)_{\text {cbow }}=\frac{1}{M} \sum_{i=1}^{M} \log \left(\mathbf{U}\left[x_{i}\right] \mid \mathbf{U}\left[x_{\text {context }}\right]\right) \\
L(s)_{\text {skip }}=\frac{1}{M} \sum_{i=1}^{M} \sum_{-k \leq j \leq k} \log \left(\mathbf{U}\left[x_{i+j}\right] \mid \mathbf{U}\left[x_{i}\right]\right)
\end{gathered}
$$

where $\mathbf{U}$ denotes the word embeddings matrix and $\mathbf{U}\left[x_{i}\right]$ is the word embedding assigned to the word $x_{i}$. Due to its surprisingly efficient architecture and unsupervised training protocol, word2vec can be trained over a large-scale unannotated corpus efficiently. In my thesis, the pre-trained word2vec vectors published by 


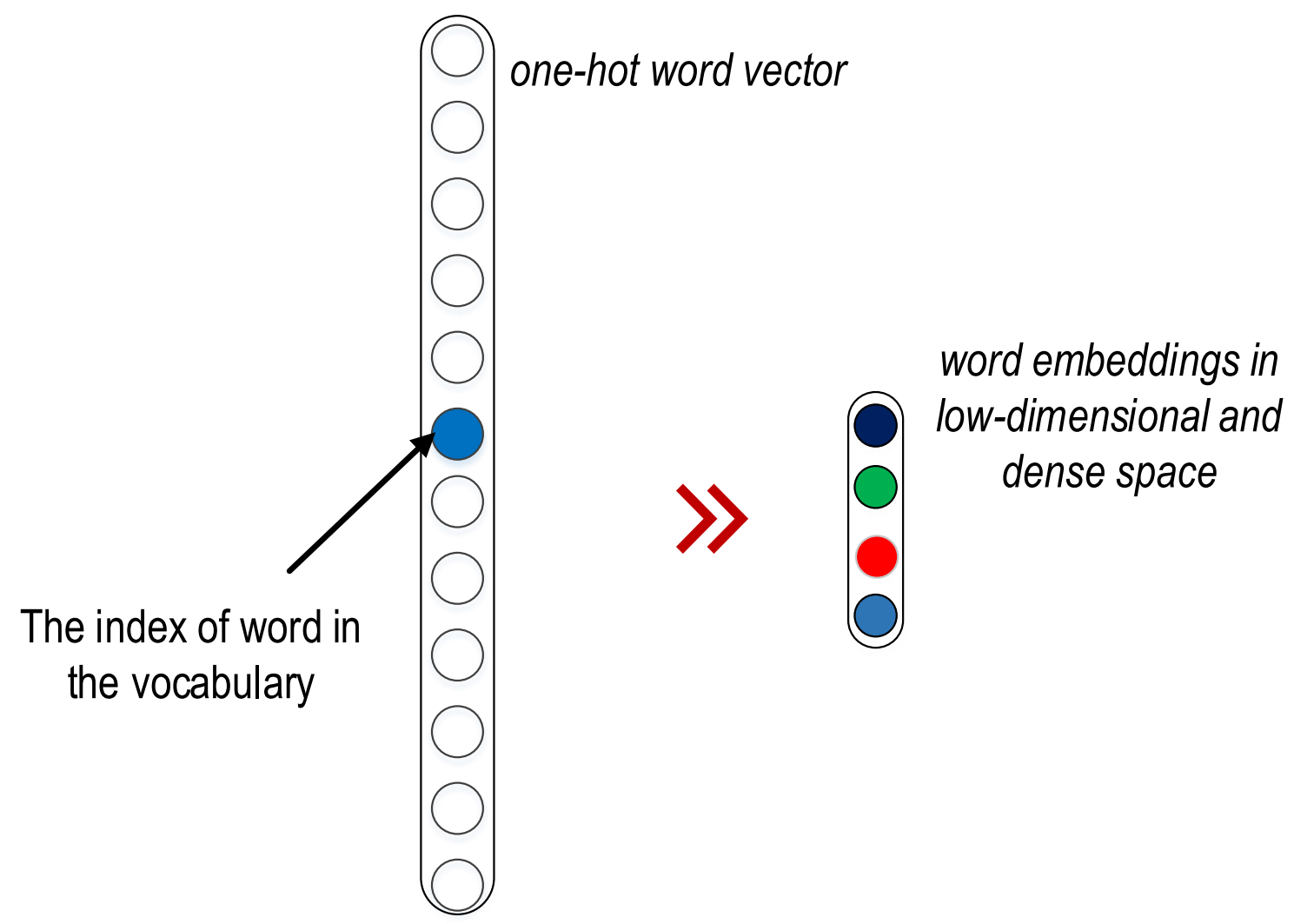

Figure 2.1: Comparison between conventional one-hot word vector and word embeddings.

\section{Output Layer \\ Hidden Layer \\ Input Layer}
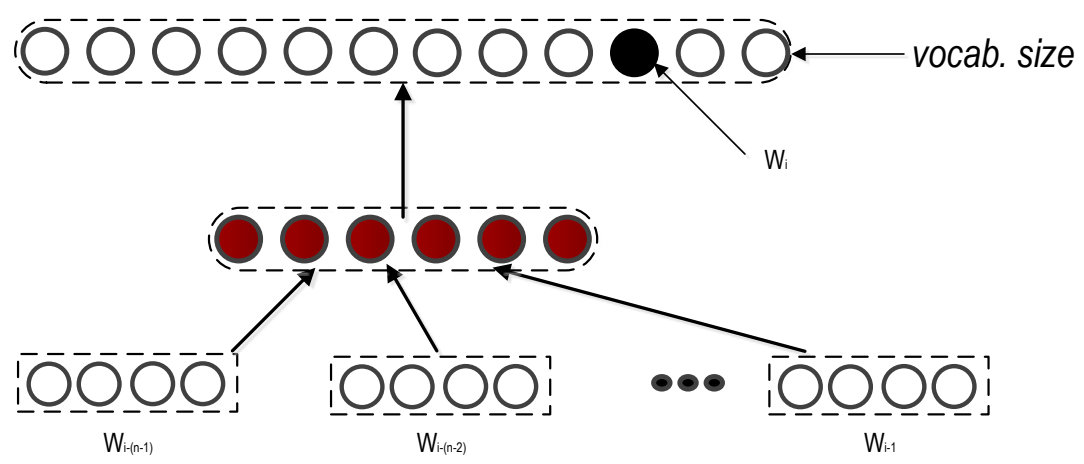

Figure 2.2: The Framework for Neural Network Language Model.

Google $^{1}$ are utilized. These word embeddings whose dimensionality is 300 were trained on a Google News corpus (over 100 billion words).

\footnotetext{
${ }^{1}$ https://code.google.com/archive/p/word2vec/
} 

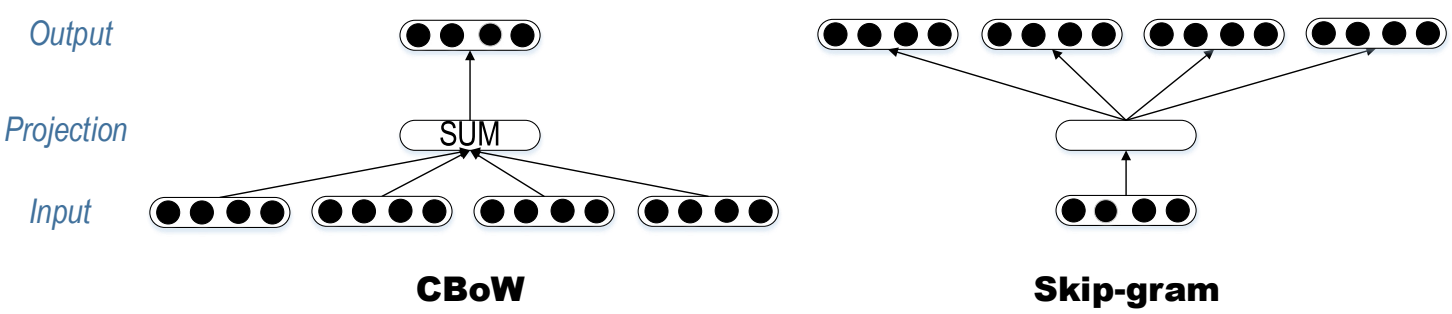

Skip-gram

Figure 2.3: The Frameworks for two word2vec models: CBoW and Skip-gram.

\subsubsection{Paragraph Vector}

Here, paragraph vector (P.V.) model that can learn distributed representation of sentences and documents is reviewed. P.V. can be regarded as a extended model of word2vec. In their original paper, two different variants of P.V. models have been proposed. One is named Distributed Bag of Words version of Paragraph Vector (PV-DBOW) as shown in Fig. 2.4, and the other is named Distributed Memory version of Paragraph Vector (PV-DM) as shown in Fig. 2.5. Compared to word2vec that word embeddings are used to make predictions about the occurrence of context and words, P.V. introduce paragraph vectors to contribute to the prediction tasks with word embeddings. PV-DBOW tries to predict words randomly sampled from the paragraph based on paragraph embeddings which corresponds to Skip-gram word2vec model, while PV-DM tries to predict the next word based on the concatenation of the paragraph vector and the prefix word vectors which corresponds to CBoW model. It is easily shown that P.V model is an unsupervised model that learns vector representations for sentences and documents.

\subsection{Semantic Compositional Models}

Semantic compositional models aim to represent larger natural language units such as phrases, sentences and documents in a vector space [54]. The compositional operation is conducted at the word-level, in which the meaning of words are also represented by vectors according to distributional information theory [55]. According to the nature of the employed composition, the compositional models can be categorized into shallow and neural models as described below. 
Classifier

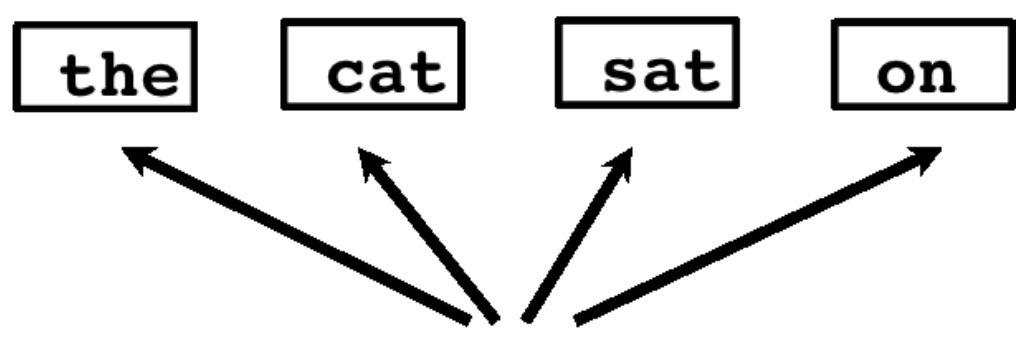

प0100

Paragraph Matrix

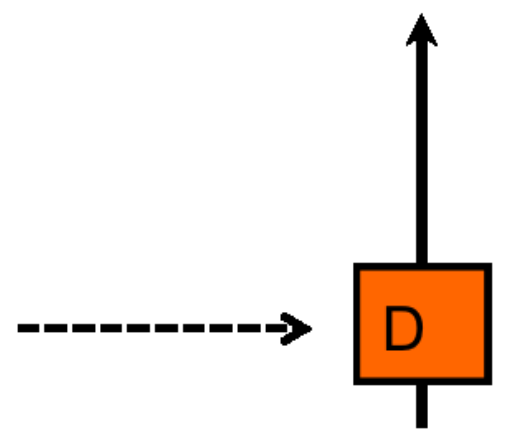

Paragraph

id

Figure 2.4: Illustrations of Distributed Bag of Words version of Paragraph Vector (PV-DBOW) in [1].

Classifier

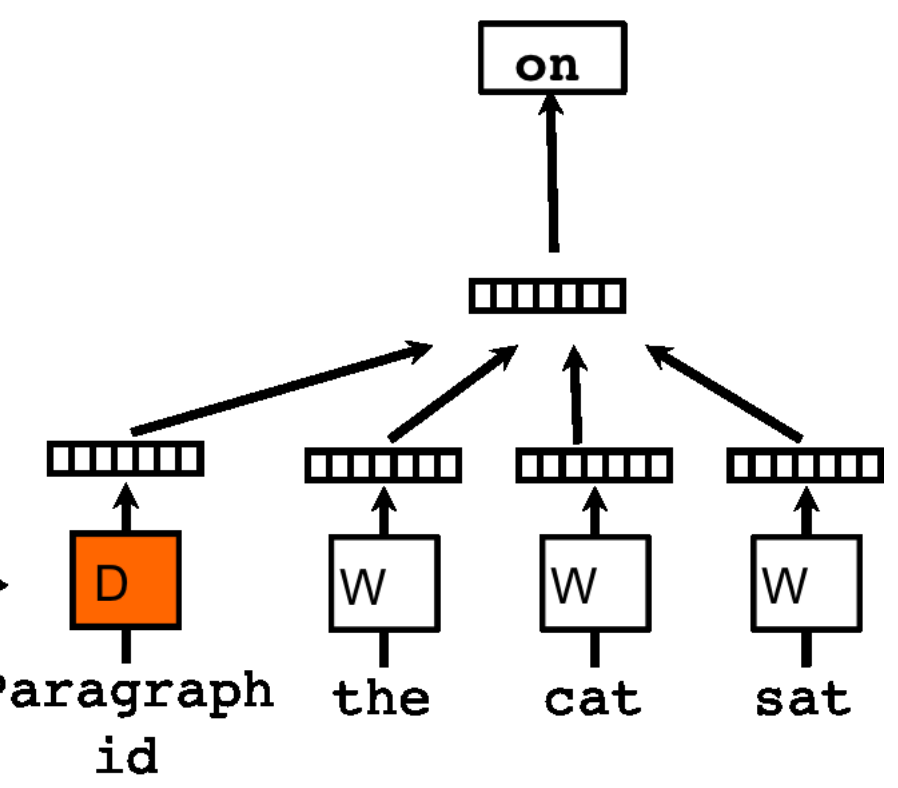

Average/Concatenate

Figure 2.5: Illustrations of Distributed Memory version of Paragraph Vector (PV-DM) in [1]. 


\subsubsection{Shallow Compositional Models}

The interaction between words is modeled by algebraic operations including vector addition, vector multiplication, and tensors operations [20-23]. In the above works, the algebraic operations are fixed. For example, average operation can be applied over all the word vectors in the sentence $h$ as follows:

$$
\overline{\mathbf{h}}=\frac{1}{l} \sum_{i=1}^{l} \mathbf{U}\left[x_{i}\right]
$$

In other works, the algebraic operations can be learned based on some supervised settings defined by syntactic relations or semantic types [56-58].

Studies have found that shallow compositional models have limitations. For example, the addition model ignores the ordering of words and hence may not capture the true semantic interactions among words.

\subsubsection{Neural Compositional Model}

With the development of deep learning, recent works have focused on neural networks of various forms [25, 28-30, 59] with a strong and robust representation learning capability. The essence of deep compositional models is the application of multi-layers neural network of various forms including recursive [24, 25], recurrent $[26,27]$ and convolutional [28-30] neural networks to learn and perform compositions over word embeddings. In more recent works, structurally more complicated neural network models including gated recursive neural network [2], directed acyclic graphs LSTM [60] and dependency-sensitivity CNN built on LSTM [61] are employed. These neural compositional models might get lost in the long-distance dependency existing in documents. Therefore, neural compositional models are extensively adopted in sentence modeling instead of document modeling. In sentence modeling, sentence is regarded as a sequence of words, in which each word is represented by the corresponding embeddings. Different neural networks employ different composition process. For example, recurrent neural networks perform composition along the word sequence one by one, while recursive neural networks perform composition along a pre-defined binary parsing tree, and convolutional 
neural networks (CNN) perform composition along sliding windows. When the composition process passes through the entire sequence, the embeddings of all words in the sentence are fused into a final and fixed-length vector to represent the sentence. Most of these neural compositional models are trained in a supervised manner, which requires a fully-connected layer as the top layer to predict targets.

Some adopted annotations are described as follows: a sentence is denoted as $s=\left(x_{1}, x_{2}, \ldots, x_{l}\right)$ with $l$ words and via looking-up the word embeddings matrix $\mathbf{U}$, each word embedding corresponding to $x_{i}$ can be obtained as $\mathbf{U}\left[x_{i}\right]$, whose dimensionality is $d$. In the following, three basic forms of neural networks are reviewed.

Convolutional Neural Networks: convolutional neural networks (CNNs) were firstly proposed by LeCun [62] for image processing, which is featured by two key properties: spatially shared weights and spatial pooling. CNN has shown their success in various computer vision applications [62-64] that input data are usually $2 \mathrm{D}$ data. CNN has also been introduced to address sequential data including Natural Language Processing and Speech Recognition [28, 65]. Generally, to address sequences, 1D convolutional layer in CNN firstly adopt multiple local filters over the whole input sequential data. Each feature map corresponding to each local filter can be generated by sliding the filter over the sequence. Then, the following pooling layer is applied to extract the most vital and fixed-length features from each feature map. And both convolution and pooling layers can be performed in a stacked way. It can be regarded that CNN model can fairly discover discriminative sub-sequence over the whole sequence. The structure of the CNN applied on a sentence is shown in Figure 2.6

The convolution operation is defined as a multiplication operation between a filter vector $\mathbf{s} \in \mathbb{R}^{m d \times 1}$ and an concatenation vector of windows of words $\mathbf{x}_{i: i+m-1}$ given by:

$$
\mathbf{x}_{i: i+m-1}=\mathbf{U}\left[x_{i}\right] \oplus \mathbf{U}\left[x_{i+1}\right] \oplus \cdots \oplus \mathbf{U}\left[x_{i-m+1}\right]
$$

where $\mathbf{x}_{i: i+m-1}$ represents a window of $m$ words starting from the word indexed as $i$. A bias term $b$ is added to the output of the convolution operation, and thus the final operation is given as:

$$
c_{i}=f\left(\mathbf{s}^{T} \mathbf{x}_{i: i+m-1}+b\right)
$$


TABLE 2.1: Equations for Nonlinear Activation Functions.

\begin{tabular}{c|c}
\hline Functions & Equations \\
\hline Sigmoid & $f(x)=\frac{1}{1+e^{-x}}$ \\
Hyperbolic Tangent & $f(x)=\frac{e^{2 x}-1}{e^{2 x}+1}$ \\
ReLu & $f(x)=\max (0, x)$ \\
\hline
\end{tabular}

where $*^{T}$ denotes the transpose of a matrix $*$ and $f$ is a non-linear activation function such as Sigmoid, Hyperbolic Tangent, or ReLu. Their equations are given in Table 2.1.

The convolution operation over the whole sequence of words is applied by sliding the window from the beginning to the ending of the sentence. Since each sentence of length $l$ has multi-windows of words $\left\{\mathbf{x}_{1: m}, \mathbf{x}_{2: m+1}, \ldots, \mathbf{x}_{l-m+1: l}\right\}$, a feature map can be obtained and the dimension of the feature map is determined by the length of the sentence $l$ and the window size $m$ :

$$
\mathbf{c}_{j}=\left[c_{1}, c_{2}, \ldots, c_{l-m+1}\right]
$$

where the index $j$ denotes the $j$-th filter.

By conducting the max-pooling operation in the feature map obtained in the convolutional layer, the single feature corresponding to the filter is obtained as:

$$
h_{j}=\max \left(\mathbf{c}_{j}\right)
$$

where $h_{j}$ corresponds to the $j$-th dimension of the output of the pooling layer. Generally, multiple filters are applied with different weights and different window sizes to derive a feature vector. The motivation behind the max-pooling operation is to filter out meaningless combinations of words. Another effect of max-pooling is that it produces a fixed-length feature vector regardless the sentence lengths.

Considering various semantic and syntactic patterns in natural language, we design multiple filters with different window sizes and randomly initialized weights to match and capture these patterns. After convolution and max-pooling operations, the produced scalar is the output of a filter applied on the entire sentence. Assuming there are $k$ filters, the output of the pooling layer is the concatenation of all outputs of the filters: 


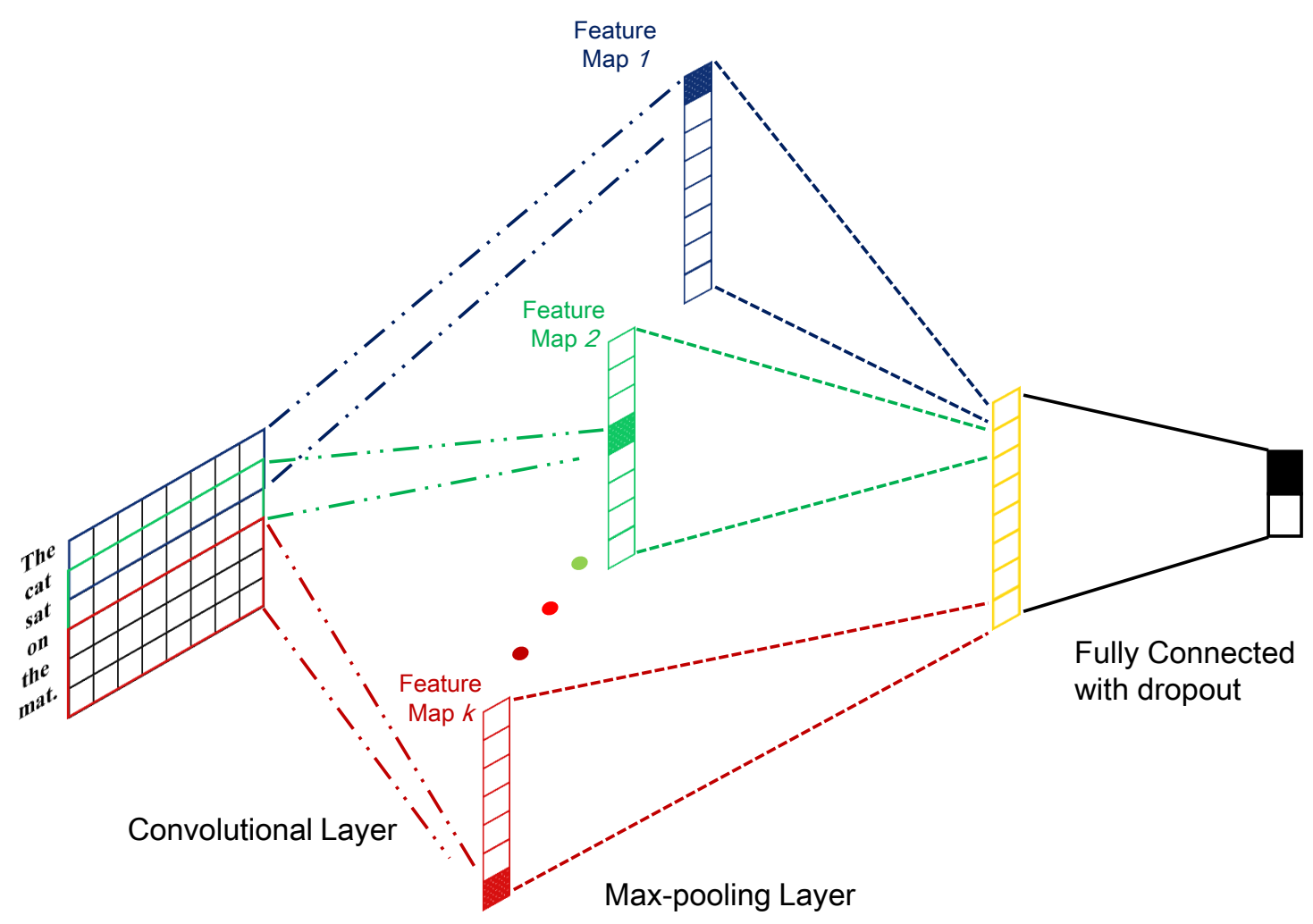

FIGURE 2.6: Illustrations of CNN for sentence representation learning.

$$
\mathbf{h}=\left[h_{1}, h_{2}, \ldots, h_{k}\right]
$$

$\mathbf{h}$ can be used as the final representation of the input sentence, if the different filters are enough to discover all meaningful structure patterns of the sentence.

Recurrent Neural Networks: As stated in [66], recurrent neural networks (RNN) are the deepest of all neural networks, which can generate and address memories of arbitrary-length sequences of input patterns. RNN is able to build connections between units from a directed cycle. Different from basic neural network: multi-layer perceptron that can only map from input data to target vectors, RNN is able to map from the entire history of previous inputs to target vectors in principal and allows a memory of previous inputs to be kept in the network's internal state. RNNs can be trained via backpropagation through time for supervised tasks with sequential input data and target outputs [67-69].

RNN can address the sentence as a sequence that word $x_{t}$ occurs at time step $\mathrm{t}$, as shown in Figure 2.7. The transition function defined in each time step $t$ takes the $t$-word vector an the previous hidden output $\mathbf{h}_{t-1}$ and update the current hidden 
output as follows:

$$
\mathbf{h}_{t}=f\left(\mathbf{W U}\left[x_{t}\right]+\mathbf{H h}_{t-1}+\mathbf{b}\right)
$$

where $\mathbf{W}$ and $\mathbf{H}$ denote transformation matrices and $\mathbf{b}$ is the bias vector. And $f$ denote the nonlinear and differentiable activation functions. After processing the whole sentence, the hidden output at the last time step i.e. $\mathbf{h}_{l}$ can be regarded as the representation of the input sentence.

Long-short Term Memory Networks: Due to the vanishing gradient problem during backpropagation for model training, conventional RNN may not capture long-term dependencies. Therefore, LSTMs were firstly presented to prevent backpropagated errors from vanishing or exploding [70-72]. Gates were introduced in LSTMs to avoid the long-term dependency problem. In addition, each recurrent unit is able to adaptively capture dependencies of different time scales via the gates functions. The core idea behind LSTMs lies that at each time step, a few gates are used to control the passing of information along the sequences that can capture long-range dependencies more accurately. In our paper, we adopt one popular LSTM framework proposed in [71]. In LSTM, at each time step $t$, hidden state $\mathbf{h}^{t}$ is updated by word embedding $\mathbf{U}\left[x_{t}\right]$ of the current word $x_{t}$, hidden state at previous time step $\mathbf{h}_{t-1}$, input gate $\mathbf{i}_{t}$, forget gate $\mathbf{f}_{t}$, output gate $\mathbf{o}_{t}$ and a memory cell $\mathbf{c}_{t}$. The following updating equations are given as follows:

$$
\begin{aligned}
\mathbf{i}_{t} & =\sigma\left(\mathbf{W}^{i} \mathbf{U}\left[x_{t}\right]+\mathbf{V}^{i} \mathbf{h}_{t-1}+\mathbf{b}^{i}\right), \\
\mathbf{f}_{t} & =\sigma\left(\mathbf{W}^{f} \mathbf{U}\left[x_{t}\right]+\mathbf{V}^{f} \mathbf{h}_{t-1}+\mathbf{b}^{f}\right), \\
\mathbf{o}_{t} & =\sigma\left(\mathbf{W}^{o} \mathbf{U}\left[x_{t}\right]+\mathbf{V}^{o} \mathbf{h}_{t-1}+\mathbf{b}^{o}\right), \\
\mathbf{c}_{t} & =\mathbf{f}_{t} \odot \mathbf{c}_{t-1}+\mathbf{i}_{t} \odot \tanh \left(\mathbf{W}^{c} \mathbf{U}\left[x_{t}\right]+\mathbf{V}^{c} \mathbf{h}_{t-1}+\mathbf{b}^{c}\right), \\
\mathbf{h}_{t} & =\mathbf{o}_{t} \odot \tanh \left(\mathbf{c}^{t}\right) .
\end{aligned}
$$

where model parameters including all $\mathbf{W} \in \mathbb{R}^{d \times k}, \mathbf{V} \in \mathbb{R}^{d \times d}$ and $\mathbf{b} \in \mathbb{R}^{d}$ are shared by all time steps and learned during model training, $\sigma$ is the sigmoid activation function, $\odot$ denotes the element-wise product, $k$ is a hyper-parameter that representing the dimensionality of hidden vectors. Here, the Eq. (2.14) defines the hidden transition functions.

Gated Recurrent Units: Recently, Gated Recurrent Units (GRUs) as a simpler variant of LSTMs were proposed [27, 73]. GRU has two gates including a reset 
gate $r$ that adjusts the incorporation of new input with the previous memory and an update gate $z$ that controls the preservation of the previous memory. The transformation equations behind GRU are given as follows:

$$
\begin{aligned}
\mathbf{z}_{t} & =\sigma\left(\mathbf{W}^{z} \mathbf{U}\left[x_{t}\right]+\mathbf{V}^{z} \mathbf{h}_{t-1}+\mathbf{b}^{z}\right), \\
\mathbf{r}_{t} & =\sigma\left(\mathbf{W}^{r} \mathbf{U}\left[x_{t}\right]+\mathbf{V}^{r} \mathbf{h}_{t-1}+\mathbf{b}^{r}\right), \\
\tilde{\mathbf{h}}_{t} & =\tanh \left(\mathbf{W}^{c} \mathbf{U}\left[x_{t}\right]+\mathbf{V}^{c}\left(\mathbf{r}_{t} \odot \mathbf{h}_{t-1}\right)\right), \\
\mathbf{h}_{t} & =\left(1-\mathbf{z}_{t}\right) \odot \mathbf{h}_{t-1}+\mathbf{z}_{t} \odot \tilde{\mathbf{h}}_{t} .
\end{aligned}
$$

Besides these proposed advanced transition functions such as LSTMs and GRUs, multi-layer and bi-directional recurrent structure can increase the model capacity and flexibility. As shown in Figure 2.8.(b), multi-layer structure can enable the hidden output of one recurrent layer to be propagated through time and used as the input data to the next recurrent layer. And the bidirectional recurrent structure is able to process the sequence data in two directions including forward and backward ways with two separate hidden layers, which is illustrated in Figure 2.8.(c). The following equations define the corresponding hidden layer function and the $\rightarrow$ and $\leftarrow$ denote forward and backward process, respectively.

$$
\begin{aligned}
\overrightarrow{\mathbf{h}}_{t} & =\mathbb{H}\left(\overrightarrow{\mathbf{W}} \mathbf{U}\left[x_{t}\right]+\overrightarrow{\mathbf{H}} \overrightarrow{\mathbf{h}}_{t-1}+\overrightarrow{\mathbf{b}}\right) \\
\overleftarrow{\mathbf{h}}_{t} & =\mathbb{H}\left(\overleftarrow{\mathbf{W}} \mathbf{U}\left[x_{t}\right]+\overleftarrow{\mathbf{H}} \overleftarrow{\mathbf{h}}_{t-1}+\overleftarrow{\mathbf{b}}\right)
\end{aligned}
$$

Normally, the complete bi-directional RNN hidden element representation $\mathbf{h}^{t}$ is the concatenated vector of the outputs of forward and backward processes as follows:

$$
\mathbf{h}_{t}=\overrightarrow{\mathbf{h}}_{t} \oplus \overleftarrow{\mathbf{h}}_{t}
$$

Recursive Neural Networks: Different from recurrent neural networks that take composition along the sequence of words, recursive neural networks perform the composition function along a pre-defined binary parsing tree. The structure of recursive neural networks is shown in Figure 2.9. As shown in Figure 2.9, a parsing tree is an ordered, rooted tree that represents the syntactic structure of a sentence according to some predefined grammar. The update function is given as

$$
h=f\left(\mathbf{W}_{L} \mathbf{U}\left[x_{l}\right]+\mathbf{W}_{R} \mathbf{U}\left[x_{r}\right]+b\right)
$$




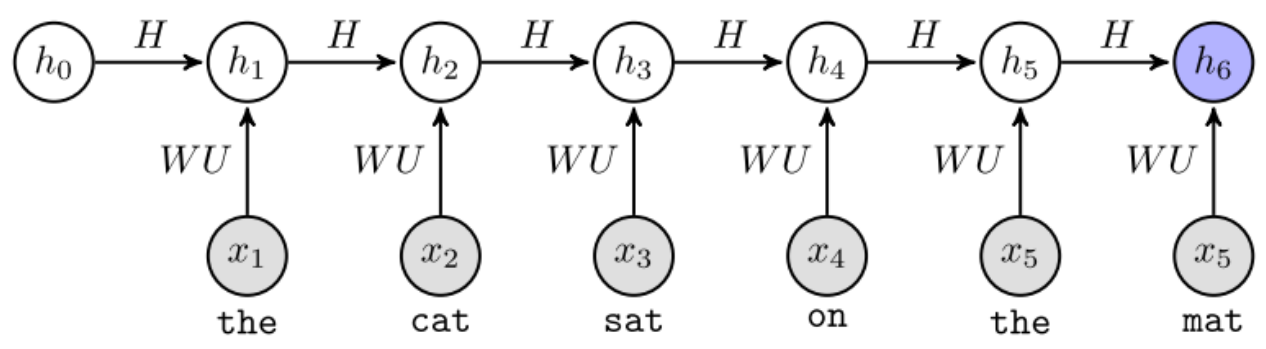

FiguRE 2.7: Illustrations of Recurrent Neural Networks for sentence representation learning.

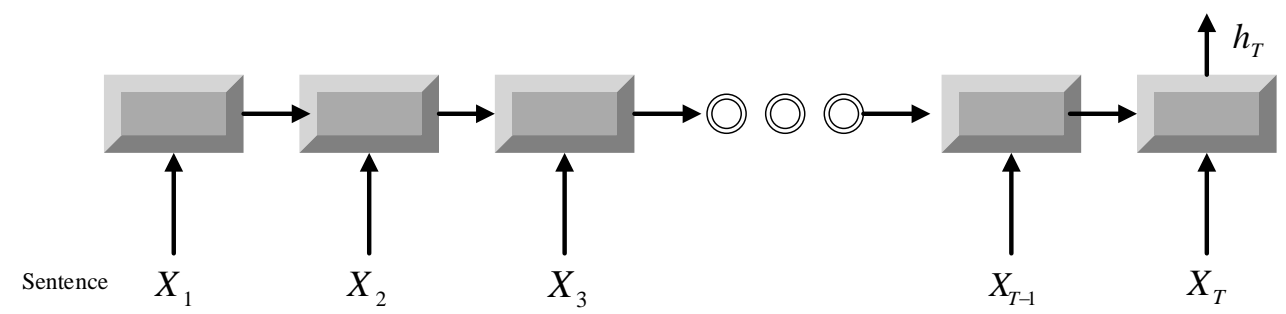

(a) Basic RNN

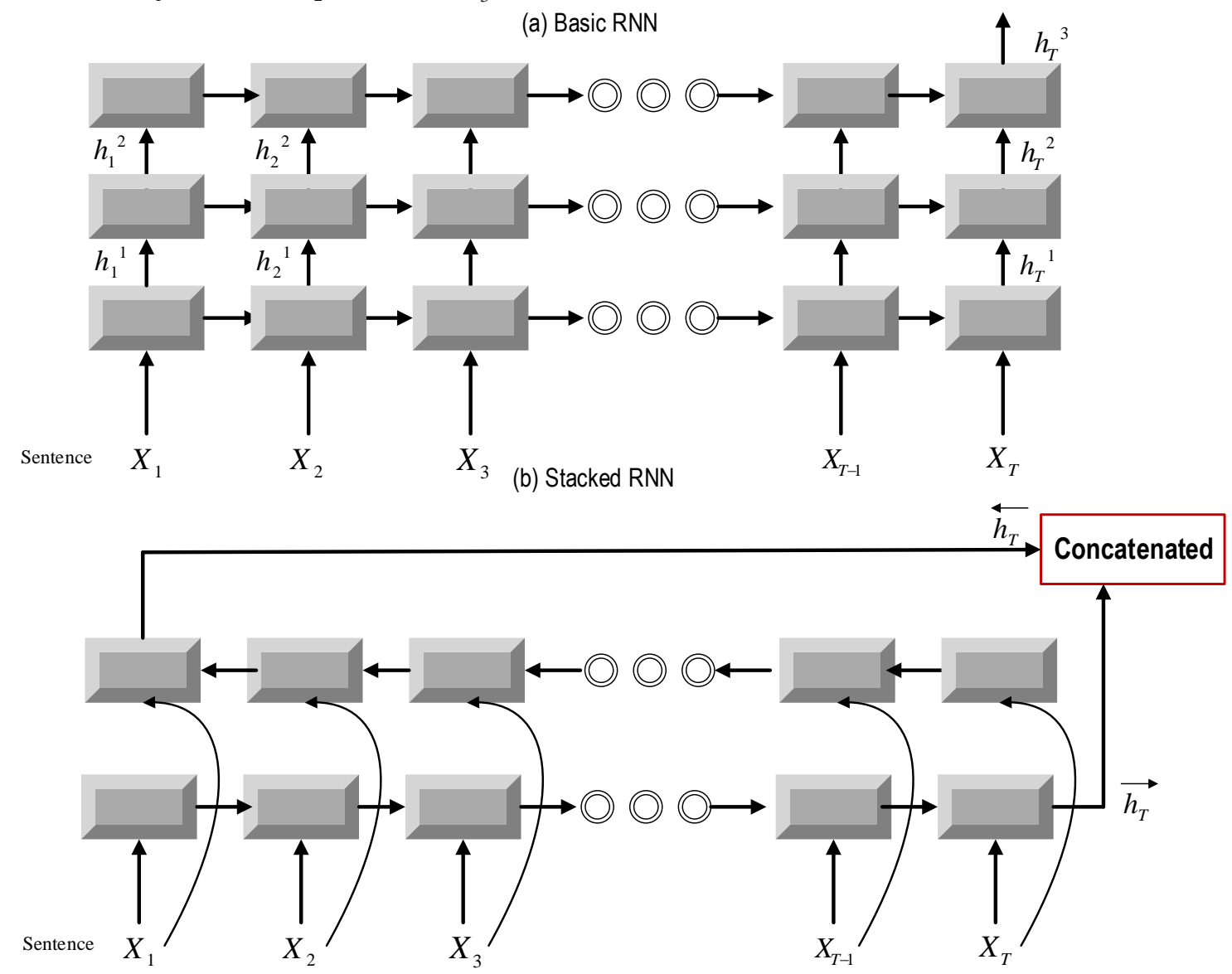

(c) Bidirectional RNN

FIGURE 2.8: Illustrations of normal RNN, stacked RNN and bidirectional RNN. 


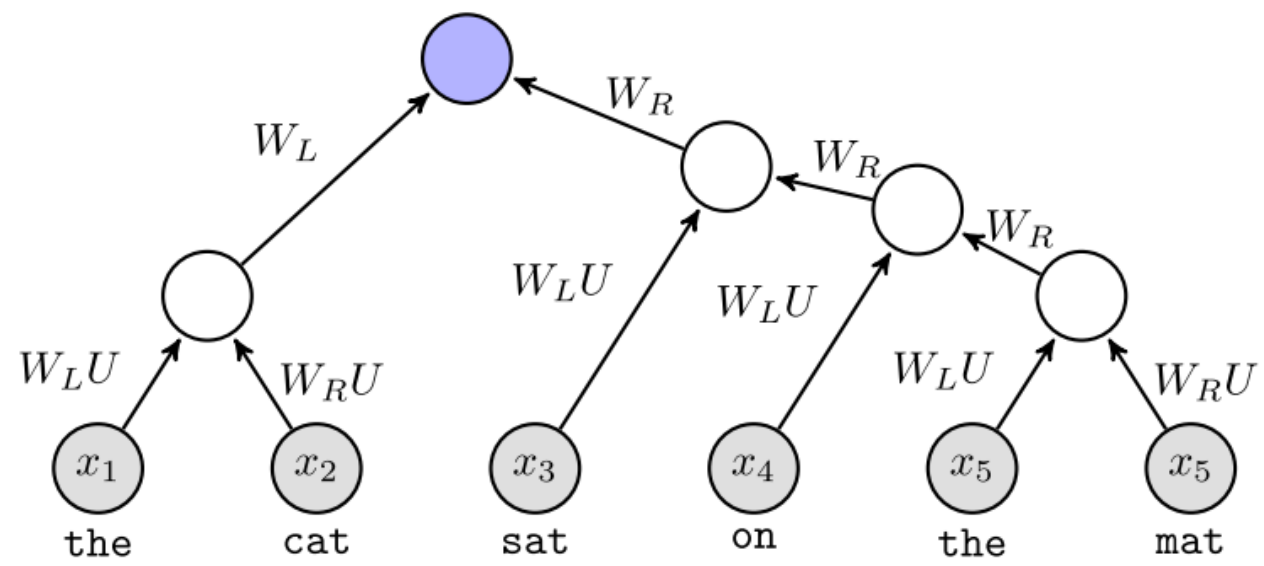

Figure 2.9: Illustrations of Recursive Neural Networks for sentence representation learning.

where $x_{l}$ and $x_{r}$ forms two leaf nodes in the tree. Since our following works are not based on this recursive structure, this framework is only briefly explained here. Previous work [74] can be referred for further reading of the application of recursive neural network on natural language processing.

Self-Adaptive Hierarchical Sentence Model: Here, a recent work named selfadaptive hierarchical sentence model (AdaSent) is reviewed. In [2], a structure of recursive gated local composition of adjacent segments has been designed to form a hierarchy of multi-scale representation for sentence. As shown in Fig. 2.10, the strucutre of AdaSent is a directed acylic graph that has $T$ levels for a input sentence of length $T$. The recursive local composition function adopted in the $t$-th level of AdaSent is defined as:

$$
\begin{gathered}
h_{j}^{t}=w_{l} h_{j}^{t-1}+w_{r} h_{j+1}^{t-1}+w_{c} \bar{h}_{j}^{t} \\
\bar{h}_{j}^{t}=f\left(\mathbf{W}_{L} h_{j}^{t-1}+\mathbf{W}_{R} h_{j+1}^{t-1}+b_{W}\right)
\end{gathered}
$$

where the range of $j$ is from 1 to $T-t+1 . \mathbf{W}_{L} \in \mathbb{R}^{D \times D}$ and $\mathbf{W}_{R} \in \mathbb{R}^{D \times D}$ are both the hidden transformation matrices, and $b_{W} \in \mathbb{R}^{D}$ is a bias vector. The intuition idea behind Eq. (2.20) lies that part of semantic meaning of a phrase of length $t$ should be derived by combing the semantics of its left $t-1$ prefix and its right $t-1$ suffix. Then, in Eq. (2.19), a gated function is introduced that $w_{l}, w_{r}$ and $w_{c}$ are 
three non-negative gating coefficients that $w_{l}+w_{r}+w_{c}=1$. Therefore, the final semantic meaning is a weighted combination of three parts including the semantic meanings of its $t-1$ prefix, $t-1$ suffix and the composition of these above two parts as defined in Eq. (2.20). Here, the softmax function is adopted to implement the gating mechanism as follows:

$$
\begin{gathered}
\left(\begin{array}{c}
w_{l} \\
w_{r} \\
w_{c}
\end{array}\right)=\operatorname{softmax}\left(G_{L} h_{j}^{t-1}+G_{R} h_{j+1}^{t-1}+b_{G}\right) \\
\operatorname{softmax}(\mathbf{v})=\frac{1}{\sum_{i=1}^{l} \exp \left(v_{i}\right)}\left(\begin{array}{c}
v_{1} \\
\vdots \\
v_{l}
\end{array}\right)
\end{gathered}
$$

where $G_{L}, G_{R} \in \mathbb{R}^{3 \times D}$ and $b_{G} \in \mathbb{R}^{3}$ are shared in the whole structure. At last, a pooling operation can be applied on each level to obtain one representation of all consecutive phrases in each level. And the outputs of each level can be regarded as representation for the input sentence. In their paper, a gating network has been designed that takes all these outputs of these levels as input and makes predictions.

\subsubsection{Analysis of Neural Compositional Models}

In recent years, neural compositional models have emerged as popular techniques for representation learning of sentence. These models normally train various forms of neural networks on top of pre-trained word embeddings using a task-specific corpus. However, most of these works neglect the multisense nature of words in the pre-trained word embeddings. Here, mutlisense means that a word may have multiple senses. Since conventional word embeddings only assign single vector to each word, multi-sense embeddings should represent each word by multiple sense-specific embeddings $[75,76]$. Therefore, in Chapter 4, we present our topic-aware neural composition models that introduce topic models to enrich the word embeddings capturing multi-senses of words.

In addition, most of these models are trained in a supervised manner, with a fullyconnected layer at the top of the neural network. And compared to conventional text representation learning models, these neural compositional models usually 


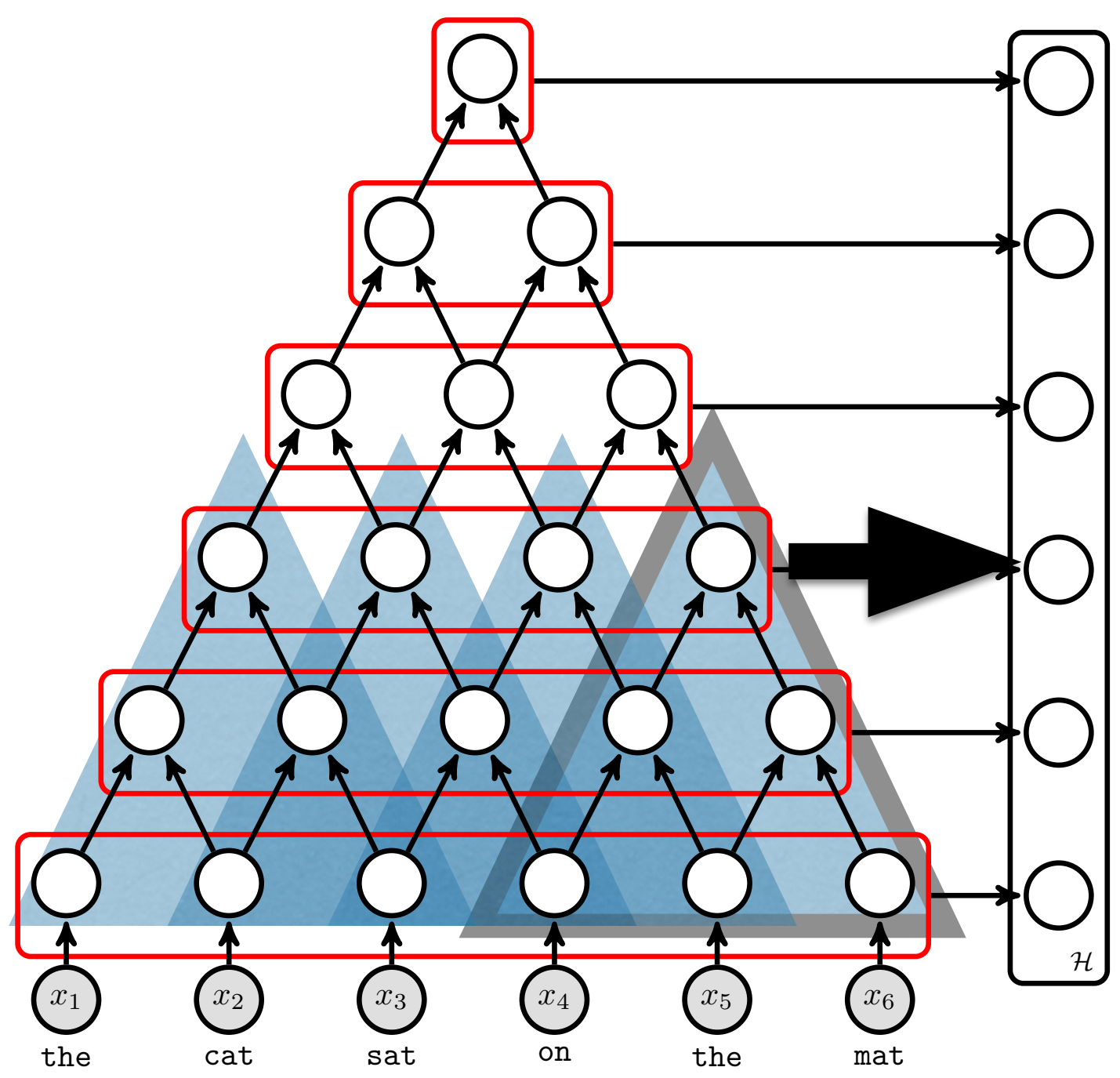

FiguRE 2.10: Illustrations of Self-adaptive Hierarchical Sentence Model for sentence representation learning in [2].

have a huge number of model parameters. Therefore, they have several limitations including heavy computation, demands of sufficient and high-quality annotated training corpus, and tricky hyperparameter settings. In addition, they might get lost in the long-distance dependency existing in documents. These neural compositional models are extensively adopted in sentence modeling instead of document modeling. Therefore, our works in Chapter 3 and Chapter 5 can serve as powerful alternative methods to these neural composition models in document modeling. 



\section{Chapter 3}

\section{Semi-Random Projection for Document Representation}

In this chapter, we will investigate feature extraction methods on BoW features, i.e BoW-based models. A new dimensionality reduction framework, named semirandom projection (SRP) is presented here. Several text classification tasks have been conducted to verify the effectiveness and efficiency of our proposed SRP algorithm.

The rest of the chapter is structured as follows. In Section 3.1, we formally define our problem and briefly present our motivation. Our proposed dimensionality reduction method is presented in Section 3.2. Then, we describe the stacked SRP in Section 3.3. A brief summary of our proposed approach is given in Section 3.4. In Section 3.5, we present and discuss the experimental results. Finally, concluding remarks are provided in Section 3.6.

\subsection{Background}

\subsubsection{Motivations}

As stated in section 2.1.2, Random Projection (RP) is a popular technique of dimensionality reduction for high-dimensional data because of its high computational efficiency. Compared to other linear dimensionality reduction methods such 
as PCA and LDA, RP is much less expensive in computational cost. However, RP may not produce discriminative low-dimensional feature space for pattern classification tasks since the random transformation matrix of RP is totally data independent. The latent space of RP is randomly generated without considering the structure of the original data. Therefore, RP may not capture the discriminative information underlying the original data, which is preferred in data mining tasks including visualization, clustering, classification and so on. For RP, one of the typical random assignments for the transformation matrix $\mathbf{W}$ is based on an i.i.d sampling described by:

$$
w_{i, j}=\sqrt{c} \begin{cases}1 & \text { with prob. } \frac{1}{2 c} \\ 0 & \text { with prob. } 1-\frac{1}{c} \\ -1 & \text { with prob. } \frac{1}{2 c} .\end{cases}
$$

where $c$ is set to $\sqrt{d}$. To demonstrate the limitation of RP, Figure 3.1 shows a simple 4-D example on a 2-D subspace, where the positive and negative samples are denoted by red and blue circles, respectively. According to Eq. (3.1), only two features out of the original four features will contribute to the one dimension in the reduced-dimensional space. The projection vector found by RP will be $[1-1]$, since the assignment probabilities of 1 and -1 are equal as $\frac{1}{4}$ according to Eq. (3.1). Then, the corresponding projected direction is shown in Figure 3.1 by the dashed line. It is obvious that the data in the projected space is inseparable. However, another projected direction denoted by the solid line can preserve and even emphasize the discriminative information. The simple example illustrates the limitation of RP, and this motivates the development of the "Semi-random Projection".

Here we propose a new dimensionality reduction framework, named Semi-Random Projection (SRP), with the purpose of finding a latent space with large discriminative power while having feasible computational load. Assuming the original data dimension and the reduced dimension are $d$ and $L$ respectively, we first select $d_{s}$ features in a random way. Here, $d_{s} \ll d$. We then project the data in the space spanned by the $d_{s}$ features onto a single dimension using a transformation vector with the size of $d_{s} \times 1$. The above process is repeated $r$ times to obtain a $r$-dimensional latent space. Different from RP whose transformation vectors are generated randomly, transformation vectors in our method are learned from 


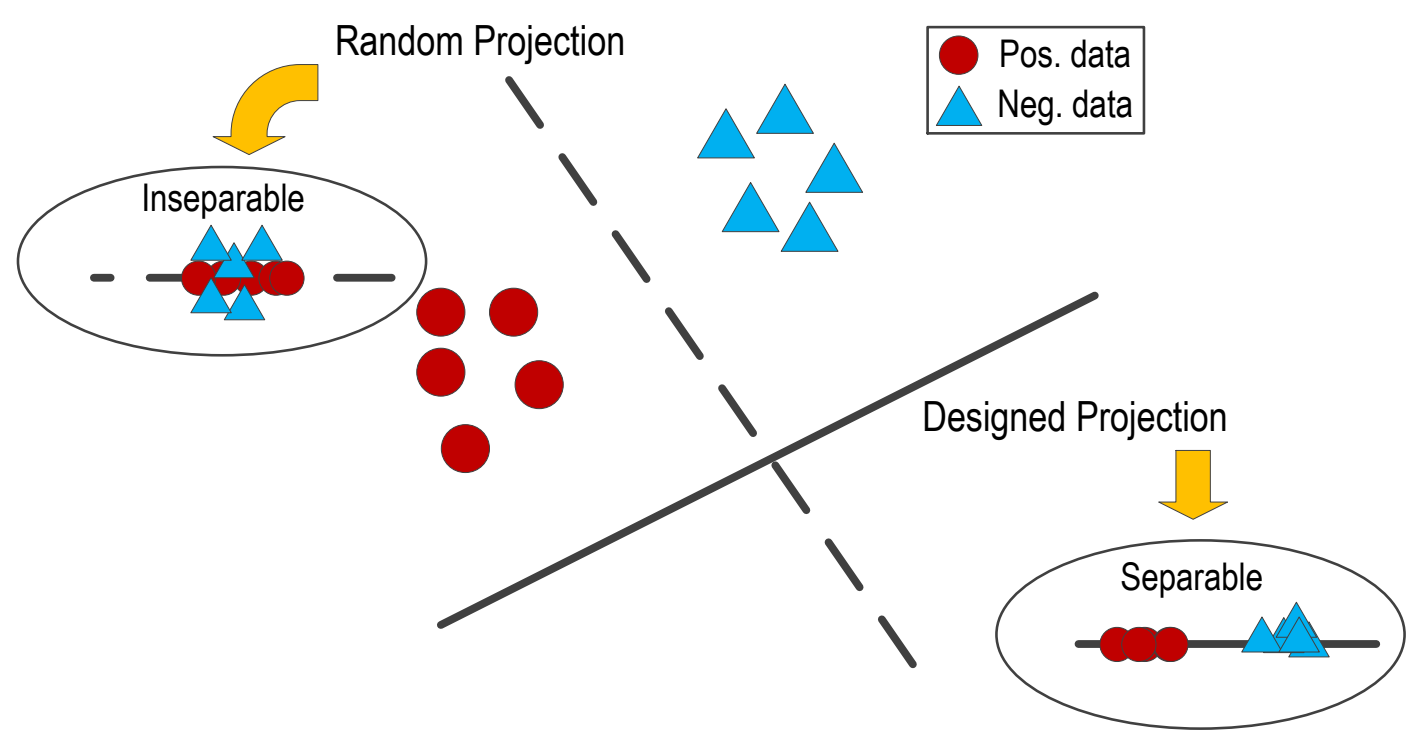

FiguRE 3.1: Illustrations of the possible ineffectiveness of Random Projection

data. The transformation vector in each iteration is computed on a $d_{s}$-dimensional sub-space, and hence demands much less computations than in the original ddimensional space. The learning scheme is proposed as: LDA is used to preserve the discriminative information of the original data.

Recently, "deep" architectures have shown to be very powerful in representation learning [77, 78]. We propose to cascade multi-layers of SRP modules to form a "deep" structure to learn new representation for data. In this stacked SRP (SSRP), the features learned by the $(l-1) t h \mathrm{SRP}$ are feeded into the $l$ th SRP as the input. In addition, the nonlinearity is applied on the output of each SRP layer. Compared to other popular deep architectures such as Stacked Denoising Autoencoder (SDA), the computation in our module SRP can be conducted in closed-form, which is able to achieve a very efficient way to implement such deep architectures [79].

\subsection{Semi-Random Projection for Dimensionality Reduction}

Motivated by the analysis in the above section, we have proposed a novel dimensionality reduction framework: semi-random projection (SRP). We want to learn a 
latent space where the beneficial task-related information can be preserved, meanwhile the running speed is comparable to that of RP. As illustrated in Eq. (3.1), approximately $\sqrt{d}$ elements in each row of the transformation matrix will be assigned 1 or -1 . To reduce the randomness of RP, these $\sqrt{d}$ elements will not be assigned 1 or -1 , but will be learned based on the data. The SRP consists of two phases: one is the random selections of sub-feature space and the other is the transformation vector learning. The term 'semi-random' originates from the fact that the first phase is random, while the second one is data-dependent. Since the transformation vector learning is based on linear discriminant analysis (LDA), a brief introduction to LDA is given firstly.

LDA is a supervised dimensionality reduction method that finds the projection by maximizing the ratio of interclass difference to intraclass scatter. Here, we will adopt the unified graph embedding framework to describe LDA [80]. The optimization problem for LDA can be summarized as following:

$$
\mathbf{W}^{*}=\underset{\mathbf{W} \in \mathbb{R}^{d \times r}}{\operatorname{argmax}} \operatorname{Tr}\left(\frac{\mathbf{W}^{T} \mathbf{L W}}{\mathbf{W}^{T} \mathbf{B W}}\right)
$$

where $\operatorname{Tr}$ denotes the trace of a matrix. Intuitively, $\mathbf{L}$ represents the quantity that we would like to enhance and $\mathbf{B}$ denotes the quantity that we would like to suppress. Different settings for $\mathbf{L}$ and $\mathbf{B}$ lead to different Dimensionality Reduction approaches. For LDA, $\mathbf{L}$ and $\mathbf{B}$ are given as follows:

$$
L D A:\left\{\begin{array}{l}
\mathbf{L}=\sum_{c=1}^{N_{c}} n_{c}\left(\overline{\mathbf{x}}^{c}-\overline{\mathbf{x}}\right)\left(\overline{\mathbf{x}}^{c}-\overline{\mathbf{x}}\right)^{T} \\
\mathbf{B}=\sum_{i=1}^{N}\left(\mathbf{x}^{i}-\overline{\mathbf{x}}^{c_{i}}\right)\left(\mathbf{x}^{i}-\overline{\mathbf{x}}^{c_{i}}\right)^{T}
\end{array}\right.
$$

where $c_{i}$ denotes the class label of the i-th sample, $\overline{\mathbf{s}}^{c}$ represents the mean vector of the c-th class, $N_{c}$ and $n_{c}$ denote the number of classes and the number of samples in the c-th class, respectively. Although the discriminative embedding space can be found by LDA, the reduced dimension is restricted to be less than the number of classes. In addition, the common drawback shared by PCA and LDA for very highdimensional data, saying the dimension is over 10000 in text mining applications, is the prohibitive computational cost, considering the cost for solving Eq. (3.2) is $\mathcal{O}\left(d^{3}\right)$. The higher the dimension of data, the heavier the computation burden for these dimensionality reduction approaches. Therefore, the incorporation of LDA into our proposed SRP framework can alleviate these potential issues. 


\subsubsection{Random Feature Subset Selection and Transforma- tion Vector Learning}

In SRP, we keep the property of RP that only a portion of weights in the transformation matrix are non-zero. And the feature subset sampling is conducted randomly.

When the random feature subset generations have been done, we need to learn the weights for these chosen attributes in transformation vectors. Assuming the number of chosen features is $d_{s}\left(d_{s}\right.$ is an integer close to $\left.\sqrt{d}\right)$, we only use these selected $d_{s}$ features to represent the data so that the original data matrix $\mathbf{X} \in \mathbb{R}^{d \times N}$ is reduced to a sub-matrix $\widehat{\mathbf{X}_{\mathbf{i}}}$ with a smaller size of $d_{s} \times N$ in the i-th iteration. The transformation vector $\mathbf{w}_{i} \in \mathbb{R}^{d_{s} \times 1}$ maps the corresponding data $\widehat{\mathbf{X}_{\mathbf{i}}}$ into the one-dimensional space as follows:

$$
\mathbf{h}_{i}=\mathbf{w}_{i}^{T} \widehat{\mathbf{X}_{\mathbf{i}}}
$$

where $\mathbf{h}_{i} \in \mathbb{R}^{1 \times N}$ is a vector, in which each element is the projection of a sample on the new dimension, and $\mathbf{w}_{i}$ is the transformation vector as the i-th column in the transformation matrix $\mathbf{W}$. We adopt LDA to derive the transformation vector that the latent space can be learned. By following the graph embedding framework as described in Section 2, the transformation vector can be learned according to Eq. (3.2), in which matrices $\mathbf{L}$ and $\mathbf{B}$ can be calculated as:

$$
T V L:\left\{\begin{array}{l}
\mathbf{L}=\sum_{c=1}^{N_{c}} n_{c}\left(\widehat{\overline{\mathbf{x}}^{c}}-\widehat{\overline{\mathbf{x}}}\right)\left(\widehat{\overline{\mathbf{x}}^{c}}-\widehat{\overline{\mathbf{x}}}\right)^{T} \\
\mathbf{B}=\sum_{i=1}^{N}\left(\widehat{\mathbf{x}^{i}}-\widehat{\mathbf{x}^{c_{i}}}\right)\left(\widehat{\mathbf{x}^{i}}-\widehat{\widehat{\mathbf{x}}^{c_{i}}}\right)^{T}+\eta \mathbf{I}_{\mathbf{d}_{\mathbf{s}}}
\end{array}\right.
$$

Where $\mathbf{I}_{\mathbf{d}_{\mathbf{s}}}$ denotes an identity matrix with a size of $d_{s} \times d_{s}$ and $\widehat{\wedge}$ means the corresponding value calculated on the randomly selected feature subset from the original high dimension space. It is shown that in SRP, LDA is conducted in the random generated low-dimensional subspace of the data, the computational cost of the learning scheme is much less than the one in the original space. Here, the second term in $B$ of Eq. (3.5) represents a regularization term.

The optimization problem in Eq. (3.2) can be reformulated as the following generalized eigenvalue problem:

$$
L \varphi=\lambda B \varphi
$$


where $\varphi$ and $\lambda$ are the eigen vector and its corresponding eigen value. The optimal transformation vector can be calculated based on the eigen vector $\varphi_{1}$ with the largest eigen value $\lambda_{1}$ as follows:

$$
\mathbf{w}_{i}=\sqrt{\lambda_{1}} \varphi_{1}
$$

where the term $\sqrt{\lambda_{1}}$ is used to normalize the linear mapping vector. Thus, the larger the eigenvalue $\lambda_{1}$, the larger the weight for the linear mapping vector $\mathbf{w}_{i}$.

\subsubsection{Algorithm Details}

An unified framework behind RP and SRP is shown in Figure 3.2. The difference between RP and SRP is that learning is used in SRP for transformation matrix from feature subsets to latent space, while random assignment is used in RP.

In SRP, a feature subset with $d_{s}$ features is firstly generated from the original $d$ features in a random way. According to Eq. (3.1), the hyperparameter $d_{s}$ can be set as: $\lfloor\sqrt{d}\rfloor$. Training and testing data are both mapped into the subspace. Then, matrices $\mathbf{B}$ and $\mathbf{L}$ in Eq. (3.5) are computed and the eigenvalue problem in Eq. (3.6) is solved. The largest eigenvalue and its corresponding eigenvector are taken to compute the transformation vector $\mathbf{w}_{i}$ from Eq. (3.7). Based on the obtained transformation vector, the data is projected onto a new dimension of the latent space, as shown in Figure 3.2. It is obvious that $\mathbf{w}_{i}$ is based on the feature subset rather than all features. To achieve an unifying representation of the transformation vector for all features, we extend the length of the transformation vector from the number of selected features $d_{s}$ to the original data dimension $d$. The elements of the new transformation vector $W_{i}$ are kept the same as the corresponding values in $\mathbf{w}_{i}$, while the other elements are set to be zero. The above procedures are repeated $r$ times, and a set of transformation vectors $\left\{W_{i}\right\}$ can be obtained. The transformation matrix $\mathbf{W} \in \mathbb{R}^{d \times r}$ can be constructed as follows:

$$
\mathbf{W}=\left(W_{1}\left|W_{2}\right| \ldots \mid W_{r}\right)
$$

The SRP framework can be named as discriminative SRP. Algorithm 1 summarizes the complete procedure for this proposed approach 


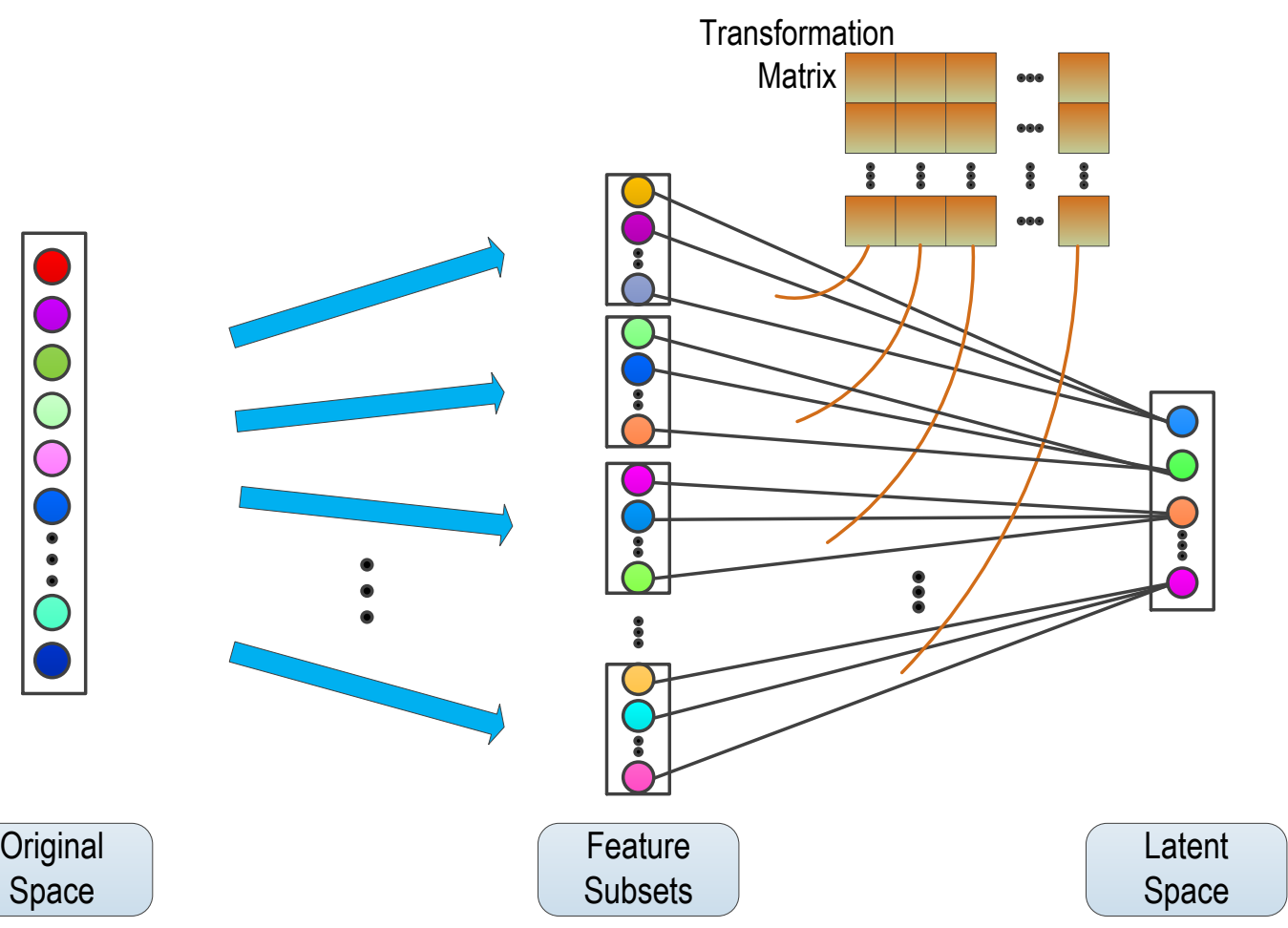

FiguRE 3.2: Unified graph explanation for Random Projection and SemiRandom Projection

\subsection{Stacked Semi-Random Projection}

Deep architectures such as stacked autoencoders (SDA) have been successfully used to learn robust features $[79,81]$. In SDA, each layer as an autoencoder is trained to learn a non-linear transformation of its input, and the output of the previous layers are used as the input of the subsequent layer. Inspired by this greedy layer-wise procedure adopted in SDA, we stack SRP by feeding the output of the $(k-1)^{\text {th }}$ SRP as the input into the $k^{\text {th }}$ SRP. In addition, the sigmoid function is applied to the output of each SRP layer to introduce nonlinearity to the learned representation. The output of the $k^{\text {th }}$ SRP layer is denoted by $\mathbf{H}^{k}$ with the dimensionality of $r^{k}$, and the input of the first layer is set to the original full data $\mathbf{H}^{0}=\mathbf{X}$ with the dimensionality of $r^{0}=d$ (the original dimensionality). In the $k^{\text {th }} \mathrm{SRP}$ layer, the transformation matrix $\mathbf{W}^{k}$ is learned by applying regularized LDA on a series of subspaces generated randomly from the previous layer's output $\mathbf{H}^{k-1}$, and the output can be calculated as: 
Algorithm 1 Semi-Random Projection

Input: data matrix of training samples $\mathbf{X} \in \mathbb{R}^{d \times N}$ and its corresponding label sets $\mathbf{y}$; number of subfeatures for each random selection $d_{s}$; reduced dimension $r$; and regularization weights $\eta$

Output: dimensionality reduced training samples: $\mathbf{H}$ and transformation matrix: W

1: for $i=1$ to $r$ do

2: $\quad$ Select $d_{s}$ features randomly from the original high features and map training data and testing data on these features to obtain $\widehat{\mathbf{X}}_{i}$

3: $\quad$ Construct matrices $\mathbf{L}$ and $\mathbf{B}$ based on $\widehat{\mathbf{X}}_{i}$ and $\mathbf{y}$ from Eq. (3.5)

4: Apply the Eigen-solver on Eq. (3.6) and derive the optimal transformation vector $\mathbf{m}_{i}$ based on Eq. (3.7)

5: $\quad$ Extend the length of vector $\mathbf{w}_{i}$ from $d_{s}$ to $d$ via setting other elements corresponding to these unselected features to be zero. The extended vector is denoted as $M_{i} \in \mathbb{R}^{d \times 1}$

6: end for

7: Construct the transformation matrix $\mathbf{W}$ according to Eq. (3.8)

8: Calculate the transformed data matrix $\mathbf{H}$ according to Eq. (3.4)

9: return $\mathbf{W}$ and $\mathbf{H}$

$$
\mathbf{H}^{k}=\operatorname{sigmoid}\left(\left[\mathbf{W}^{k}\right]^{T} \mathbf{H}^{k-1}\right)
$$

Different from SDA where each layer is trained to reconstruct the output of the previous layer, each SRP layer is trained to learn a projection with maximum discriminative power. Throughout our work, the relationship between the dimensionality of the output and the input of the $k^{\text {th }} \mathrm{SRP}$ is determined as:

$$
r^{k}=m\left\lfloor\sqrt{r^{k-1}}\right\rfloor
$$

where $m$ is a user-specified integer. In $k^{\text {th }}$ SRP layer, the number of randomly sampled features is set to $\left\lfloor\sqrt{r^{k-1}}\right\rfloor$, which means each feature of the input can be sampled approximately $m$ times at average. Therefore, the information underlying input features to each SRP layer will not be lost although random feature sampling is conducted. It is clear that the dimensionality of data is reduced layer by layer. Due to the rounding down operation $\rfloor\rfloor$ in Eq. (3.10), the dimensionality of data will be converged to a fixed number finally regardless of the original dimensionality, which can ensure the stability of the latent representation learned by this stacked structure. The final dimension is only conditioned on the number $m$. The number of layers can be large since the computation of SRP is cheap due to the low input 


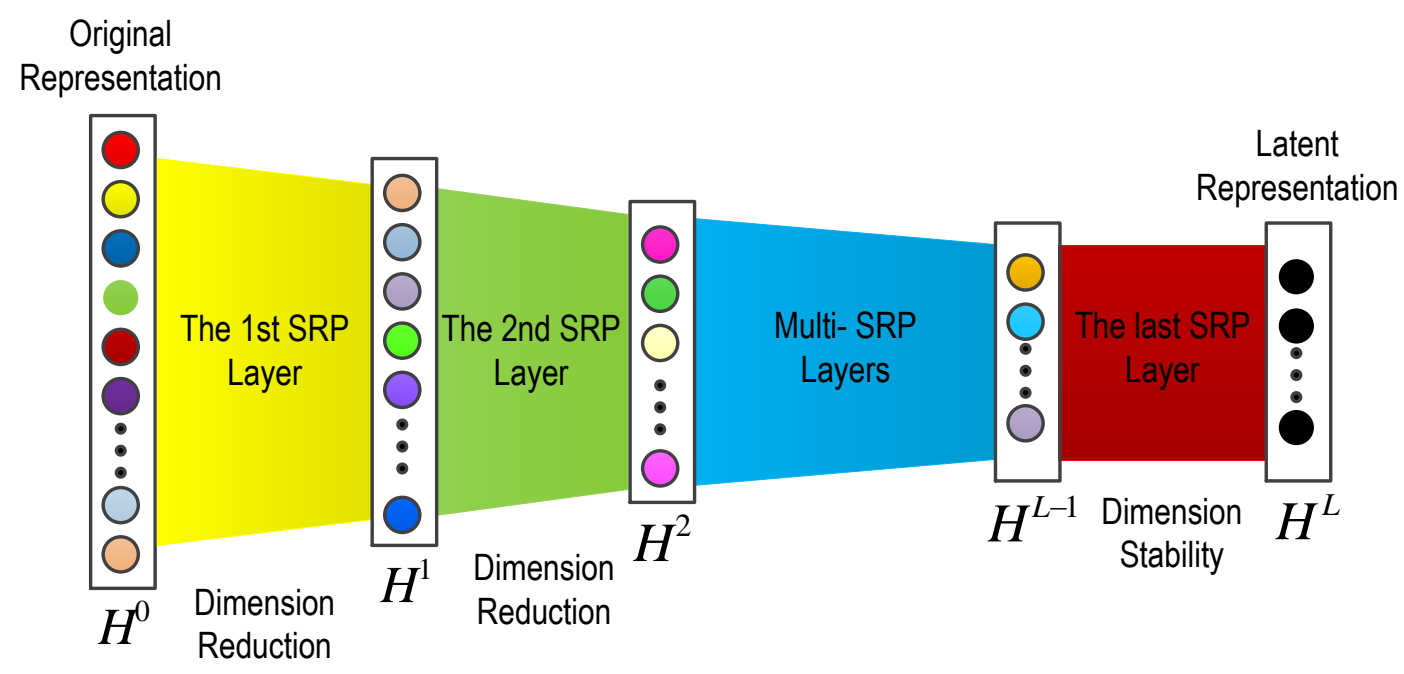

FIGURE 3.3: Architecture of Stacked SRP with $l$ layers.

dimensionality. When the stacked SRP with $L$ layers is trained, the latent representation of data is given by the output of the last layer $\operatorname{sigmoid}\left(\left[\mathbf{W}^{L}\right]^{T} \mathbf{H}^{L-1}\right)$. We refer this stacked extension of SRP as SSRP. There are three hyper-parameters in SSRP: the dimension relationship parameter $m$, the regularization parameter $\eta$ in each SRP layer and the number of SRP layers $L$. The overall structure of our proposed stacked SRP is shown in Fig 3.3.

\subsection{Discussions}

\subsubsection{Random Settings}

Compared to algorithms with parameter learning or tuning, the randomized algorithm has a very fast learning speed. Inspired by this intuition, algorithms with some degree of randomness, such as Random Projection (RP) and Extreme Learning Machine (ELM), have been proposed [45, 46, 82, 83]. However, the random components in these approaches generate parameters without considering the characteristics or useful structures underlying the data. Although a satisfying running speed can be achieved, the random settings may result in big variance in performance. To address this issue, our proposed approach tries to reduce the degree of randomness in RP and balance the computational efficiency. The experiments described in section 3.5 has proven that our proposed SRP can work with a moderate 
and acceptable speed, which is slower than RP but much faster than conventional learning approaches including PCA and LDA.

\subsubsection{Computational Complexity}

As described in the unified framework for linear dimensionality reduction, the main computational burden lies in the eigenvalue solving process in Eq. (3.6). It is known that the computational complexity of eigenvalue decomposition of a matrix is $\mathcal{O}\left(n^{3}\right)$, where $n$ is the dimension of the matrix [84]. For Principal Component Analysis (PCA) and Linear Discriminant Analysis (LDA), the dimension of the matrix in the computation is the feature number $d$, and thus the computational complexity for PCA and LDA is $\mathcal{O}\left(d^{3}\right)$ assuming $N \ll d$. PCA and LDA might be computational prohibitive for high-dimensional data in some applications, such as in text mining, where the dimensionality is easily over 10,000. In contrast, all of the computations in SRP are conducted on a low-dimensional space after the random subsampling of features. If the reduced dimension is $r$ in SRP, the complexity is approximately $\mathcal{O}\left(r d^{3 / 2}\right)$ when the number of sub-sampled features is set to $\sqrt{d}$ as proposed in RP algorithms. $\mathcal{O}\left(r d^{3 / 2}\right)$ is much less than $\mathcal{O}\left(d^{3}\right)$, when $d$ is large. As described in Section 3.5, $d$ is as high as 54,887 .

\subsubsection{Sparsity of Transformation Matrix}

In linear dimensionality reduction, the key problem is the learning of the transformation matrix $\mathbf{W} \in \mathbb{R}^{d \times r}$. Elements in the $i^{\text {th }}$ column of $\mathbf{W}$ represent the weights of original features to the $i^{t h}$ latent dimension. In traditional dimensionality reduction approaches, the transformation matrix is normally a dense one and most elements are nonzero. However, this kind of dense projection matrix lacks an interpretable and intuitive explanation for its corresponding dimensionality reduction in many data mining tasks. For example, in the latent semantic indexing, i.e. the application of PCA to text analysis, each latent feature, i.e., topic, is represented by all original features: words in the entire vocabulary, which is not consistent with the true situation that each semantic topic only consists of a few words. Therefore, several sparse projection matrix learning methods have been proposed [85, 86]. These approaches impose L1-norm constraint to PCA, which can be converted to 
the Lasso problem. There are two drawbacks for these sparse approaches: (1) Since these approaches are all based on PCA, the label information of the training domain is not utilized. As a result, the discriminative information may not be retained in the new latent space. (2) The computation burden to solve the Lasso problem is huge [87]. Different from these sparse approaches proposed in [85, 86], our proposed method generates the sparse transformation matrix through random feature subsampling. Therefore, our approach is an efficient implementation of sparsity without the need of solving the Lasso problem. Although the sparsity in our approach is introduced randomly, the performance of our approach is robust and satisfying, which has been verified in the experiments.

\subsubsection{Stacked Structures}

It has been shown that the nonlinearity and the multi-layers of autoencoders are the key factors to the robust performance of SDA [79, 88]. Our proposed stacked SRP shares these two characteristics with SDA. However, our technique stands in stark contrast to SDA. In SDA, the nonlinear function also needs to be learned, which makes the training procedures non-convex. Thus, the training of SDA can only be conducted iteratively. In our stacked SRP (SSRP), we apply the nonlinear function directly on the output of the $k^{\text {th }}$ SRP layer after the transformation matrix $\mathbf{W}^{k}$ is learned, which ensures the closed-form solution and the final nonlinear transformation at the same time. We will verify and demonstrate the effectiveness of the nonlinearity and the stacking structures adopted in our SSRP in the experiments in section 3.5 .

\subsubsection{Merits of Semi-Random Projection (SRP)}

Some important merits of the proposed approach are summarized as follows:

1. The transformation matrix can be learned based on different learning schemes. The latent space found by SRP can preserve the discriminate information. It is noted that SRP, as an effective and efficient dimensionality reduction framework, can provide a good latent space to facilitate machine learning tasks. 
2. Due to the random feature subsampling, our proposed approach is computationally efficient even for very high-dimensional data.

3. The transformation matrix is sparse. The sparsity can facilitate description and understanding of the relationships between the original space and the latent space. In addition, the computational cost of the projection operation based on the transformation matrix and the storage for the transformation matrix can be reduced significantly.

4. Deep architectures can be constructed by adopting SRP as the basic block and stacking them layer by layer for latent feature learning. Since the nonlinearity is applied after the transformation matrix is learned, the stacked SRP has a closed-form solution.

\subsection{Experiments}

In this section, we compare our proposed SRP and its stacked extension SSRP with some commonly used dimensionality reduction approaches for the task of text classification, where the dimension of text data is very high. To compare our work fairly with other dimensionality reduction methods, the binary classification problem is considered here, in which we train a linear Support Vector Machine ${ }^{1}$ (SVM) on the labeled training data and test it on the unlabeled testing data.

\subsubsection{Datasets}

To evaluate the performance of these algorithms, we conduct experiments on six datasets generated from the standard text corpora 20Newsgroups ${ }^{2}$, which comprises approximately 18,000 newsgroup posts on 20 sub-categories. We performed preprocessing on the original text data including stemming and stopwords removal. In addition, the words whose document frequency is less than 2 were also ignored. After the processing, a vocabulary consisting of the top 12000 words ordered by term frequency from the whole corpus was constructed. According to vector space model, each document $\mathbf{x}$ is represented as a vector: $\mathbf{x}=\left(x_{1}, x_{2}, \ldots, x_{12000}\right)$, where

\footnotetext{
${ }^{1}$ http://www.csie.ntu.edu.tw/ cjlin/libsvm/

${ }^{2}$ http://people.csail.mit.edu/jrennie/20Newsgroups
} 
$x_{i}$ is the tf-idf value of the word $w_{i}$ in the vocabulary [89]. For each dataset, the classification task is defined as the top category classification. We selected two topcategories and splitted them into positive data and negative data. The training dataset and testing dataset contain the almost same numbers of documents. The information about the categories contained in these six datasets are given in Table 3.1. In these six datasets, each document is represented as a 12000-dimensional vector. To show how serious the sparsity problem is, we introduce the average number of features with nonzero weights to measure the sparseness of the data. As shown in Table 3.2, the data is indeed very sparse and therefore provides a good and practical testing platform to verify the performance of our proposed approach in dealing with sparse high-dimensional data.

In addition, we also introduce the farm ads data set published in [90], which consists of text ads covering various farm animal related topics crawled from twelve websites. And these ads are labeled by the corresponding content owners based on whether or not they are appropriate. For simplicity, the processed dataset is utilized directly, in which each ad is represented as a vector with the dimensionality of 54887. We randomly selected 2071 instances as the training samples and the rest 2072 ads as the testing samples. The detailed information about this farm ads data set is also shown in Table 3.2.

\subsubsection{Evaluation Metrics}

Our approach is aimed at handling high-dimensional data, therefore the evaluation criteria include two parts: one is classification error, i.e., the fraction of missclassified instances, and the other is running time. The simulation environments for all algorithms performed in our experiment are described as follows: MATLAB R2012a software environment running in with Intel Core ${ }^{\mathrm{TM}}$ i5-2400 Processor, 3.10 GHZ with 8-GB RAM. The algorithms' running time is calculated by the MATLAB function: "cputime".

\subsubsection{Compared Approaches}

As stated above, Liner SVM is adopted as the standard classifier. Since the dataset here is very sparse, the sparsity problem of the projected data in SRP space may 
TABLE 3.1: Six datasets generated from 20Newsgroups

\begin{tabular}{|c|c|c|}
\hline Dataset & Positive Examples & Negative Examples \\
\hline comp vs rec & $\begin{array}{c}\text { comp.graphics } \\
\text { comp.sys.ibm.pc.hardware } \\
\text { comp.os.ms-windows.misc } \\
\text { comp.sys.mac.hardware }\end{array}$ & $\begin{array}{l}\text { rec.autos } \\
\text { rec.sport.hockey } \\
\text { rec.motocycles } \\
\text { rec.sprot.baseball }\end{array}$ \\
\hline comp vs sci & $\begin{array}{c}\text { comp.os.ms-windows.misc } \\
\text { comp.sys.ibm.pc.hardware } \\
\text { comp.graphics } \\
\text { comp.sys.mac.hardware }\end{array}$ & $\begin{array}{c}\text { sci.crypt } \\
\text { sci.med } \\
\text { sci.electronics } \\
\text { sci.space } \\
\end{array}$ \\
\hline comp vs talk & $\begin{array}{c}\text { comp.os.ms-windows.misc } \\
\text { comp.sys.ibm.pc.hardware } \\
\text { comp.graphics } \\
\text { comp.sys.mac.hardware }\end{array}$ & $\begin{array}{c}\text { talk.politics.mideas } \\
\text { talk.politics.misc } \\
\text { talk.politics.guns } \\
\text { talk.religion.misc }\end{array}$ \\
\hline rec vs sci & $\begin{array}{l}\text { rec.autos } \\
\text { rec.sport.baseball } \\
\text { rec.motorcycles } \\
\text { rec.sport.hockey }\end{array}$ & $\begin{array}{c}\text { sci.crypt } \\
\text { sci.med } \\
\text { sci.electronics } \\
\text { sci.space } \\
\end{array}$ \\
\hline rec vs talk & $\begin{array}{l}\text { rec.autos } \\
\text { rec.sport.baseball } \\
\text { rec.motorcycles } \\
\text { rec.sport.hockey }\end{array}$ & $\begin{array}{c}\text { talk.politics.mideast } \\
\text { talk.politics.misc } \\
\text { talk.politics.guns } \\
\text { talk.religion.misc }\end{array}$ \\
\hline sci vs talk & $\begin{array}{c}\text { sci.crypt } \\
\text { sci.med } \\
\text { sci.electronics } \\
\text { sci.space }\end{array}$ & $\begin{array}{l}\text { talk.politics.misc } \\
\text { talk.religion.mis } \\
\text { talk.politics.guns } \\
\text { talk.politics.mideast }\end{array}$ \\
\hline
\end{tabular}

TABle 3.2: Statistical Properties of the seven datasets. The first six datasets are from 20Newsgroups and the final one is collected from Farm Ads.

\begin{tabular}{|c|c|c|c|c|c|c|}
\hline \multirow{2}{*}{ Dataset } & \multirow{2}{*}{ Fea. No. } & \multirow{2}{*}{ Doc. No. } & \multicolumn{2}{|c|}{ Training Datasets } & \multicolumn{2}{c|}{ Testing Datasets } \\
\cline { 4 - 7 } & & & Doc. No. & Sparsity & Doc. No. & Sparsity \\
\hline comp vs rec & 12000 & 7882 & 3940 & 41 & 3942 & 41 \\
comp vs sci & 12000 & 7855 & 3927 & 47 & 3928 & 49 \\
comp vs talk & 12000 & 7156 & 3578 & 56 & 3578 & 55 \\
rec vs sci & 12000 & 7931 & 3965 & 48 & 3966 & 46 \\
rec vs talk & 12000 & 7232 & 3615 & 54 & 3617 & 54 \\
sci vs talk & 12000 & 7205 & 3602 & 62 & 3603 & 60 \\
\hline farm ads. & 54877 & 4143 & 2071 & 193 & 2072 & 201 \\
\hline
\end{tabular}


still exist. Therefore, PCA is applied on the projected data in SRP to obtain a non-sparse data. Since PCA is conducted on a low-dimensional space, the extra computation induced is negligible. The following methods are compared:

* Raw: Original BoW feature is used.

* PCA: Data is processed by PCA. We project data onto a low-dimensional space with PCA. Then, linear SVM is applied.

* Sparse $\mathrm{PCA}^{3}$ : Sparse PCA is applied to the datasets. After projected by the sparse transformation matrix, data is classified by the linear SVM classifier.

* RP: Random Projection is adopted to map data into a low-dimensional space. Then, we use Linear SVM to classify the projected data.

* RP followed by PCA: PCA is performed on the latent space found by RP to reduce the sparsity of the data, and then, Linear SVM is applied to the low dimensional space derived by RP plus PCA. This setting is designed corresponding to our proposed SRP followed by PCA so that the effectiveness of SRP can be tested fairly.

* mSDA : marginalized stacked denoising autoencoder [88] realizes an efficient training of Stacked denoising autoencoder. The robust representation learned by mSDA is fed into Linear SVM for classification.

* SRP followed by PCA: SRP is used to learn a discriminative latent space and PCA is used subsequently to reduce the sparsity of data. At last, Linear SVM is performed to classify data with the new representation.

* SSRP: Stacked SRP is trained. Then, the output of the last layer forms the latent representation. The Linear SVM is applied on the transformed data.

Here, some user-specified parameters in these approaches are defined as follows: In SRP, the number of features selected during each random feature sampling $d_{s}$ is set to $\lfloor\sqrt{d}\rfloor$, where $d$ denotes the original dimension. SRP is adopted here to reduce the dimension of data to $10\lfloor\sqrt{d}\rfloor$ firstly. Then, PCA is applied to reduce the dimension of data to 100 further. The regularization parameters $\eta$ in SRP

${ }^{3}$ The code for sparse PCA has been kindly provided at
http://www2.imm.dtu.dk/projects/spasm/


and each layer of SSRP are determined with 5-fold cross-validation on the training data. Here, the regularization parameters in different layers of SSRP are unified as a single value. The dimension relationship parameter in SSRP $m$ is also unified to 10. Another hyper-parameter in SSRP: the number of layers $L$ is set to 20 . For other dimensionality reduction methods, the dimension of the latent space $r$ is set to 100, considering the dimension reduced by SSRP will be converged into 100 when $m=10$ in Eq. (3.10). And for fair comparison, the nonzero entries in each column of projection matrix in Sparse PCA is set to the number of subsampled features in our proposed approaches. For mSDA ${ }^{4}$, we adopt the high-dimensional extension of this algorithm, which is more efficient for high-dimensional data. The hyperparameters for mSDA are chosen as follows: the noise intensity is set to 0.5 and the number of features in each partition for high-dimensional settings is 3,000 . In SDA, the final representation is a concatenation of the original input and the output of each layer. Since the original dimension is very high, we set the number of layer to 1 to avoid an extreme high-dimensional vector and hence very high training cost for the subsequent classifier learning process.

\subsubsection{Performance Comparison on all Datasets}

The experimental results on these seven datasets are shown in Table 3.3 and Table 3.4. Since random assignments are adopted in RP, RP followed by PCA and our proposed approaches including SRP and SSRP, ten trials are conducted for these four methods on each dataset. The average classification accuracy and its corresponding standard variance are taken. In addition, the average running time is also given. As can be seen, our proposed approaches including SRP and SSRP achieve better classification performance in all of these experiments than other random approaches. The performance of SRP is only a bit inferior to that of PCA. Except for rec vs talk dataset, SSRP outperforms all other methods and has much lower computational cost than PCA. As shown in Table 3.3, SRP and SSRP significantly speed up the feature learning of PCA by one order of magnitude.

As expected, RP has the best performance on running speed but the worst one on classification accuracy. On the other hand, among all these four random algorithms including RP, RP followed by PCA and our proposed approach with two different

\footnotetext{
${ }^{4}$ The code for mSDA has been kindly provided at http://www.cse.wustl.edu/ mchen
} 
weight learning schemes, our approaches have smaller variance in the classification accuracy in almost all experiments due to the reduced degree of randomness compared to other random algorithms, at the cost of longer running time. This result also suggests that sparse PCA requires the longest running time among all compared approaches due to the solving of the Lasso problem. Sparse PCA performs not well in the classification task since sparse PCA is an unsupervised feature extraction method that may lose the discriminative information underlying training data.

It is shown that our proposed SSRP outperforms one state-of-the-art deep learning method: mSDA. The average classification accuracy of SSRP on the the total 7 datasets achieves a significant improvements of $1.6 \%$ compared to mSDA. Stacked Denoising Autoencoders (SDA) focus on robust representation learning, which have been applied successfully in many scenarios [79, 88]. However, its performance is limited by high computational cost and lack of scalability to high-dimensional features. Although mSDA alleviates the two problems, the training time of mSDA is still much longer than that of SSRP in our experiments. It should be noted that the feature concatenation operation in mSDA imposes a huge burden on the subsequent classification process.

It is also noted that SSRP performs better than SRP. This may be explained that the stacking of multiple layers can improve the performance. Such a "deep" learning structure can result in a robust and general feature learning, and thus, the linear SVM trained on the feature learned by SSRP can outperform other methods. It is noted that in each layer of SSRP, the random subsampling of features is conducted and training procedures are convex, which results in a very efficient implementation of deep architectures. In the following section, we investigate the influence of the number of layers on the data representation learned by SSRP experimentally.

To perform error analysis, paired $t$-test was conducted to compare population means of two approaches. Based on average performance, SSRP as our proposed method and PCA and mSDA as the most competitive ones were selected for paired $t$-test. We have seven datasets and seven corresponding results. The difference of mean accuracy and standard deviation of 7 results was computed as $\bar{d}$ and $\sigma_{d}$, respectively. Then, the $t$-statistic was calculated as:

$$
t=\frac{\bar{d}}{\sigma_{d} / \sqrt{n}}
$$


TABLE 3.3: Performance comparison for different datasets on running time (s).

\begin{tabular}{|c|c|c|c|c|c|c|c|c|}
\hline Dataset & Raw & PCA & Sparse PCA & RP & RP+PCA & mSDA & SRP+PCA & SSRP \\
\hline comp vs rec & 189 & 453 & 5012 & 9 & 24 & 75 & 31 & 27 \\
comp vs sci & 208 & 442 & 5031 & 10 & 27 & 95 & 32 & 27 \\
comp vs talk & 150 & 398 & 4588 & 8 & 20 & 177 & 28 & 24 \\
rec vs sci & 221 & 458 & 4942 & 12 & 26 & 183 & 32 & 27 \\
rec vs talk & 176 & 395 & 4226 & 9 & 22 & 70 & 28 & 24 \\
sci vs talk & 182 & 395 & 4226 & 8 & 23 & 142 & 29 & 26 \\
Farm Ads. & 233 & 594 & 3884 & 40 & 137 & 3884 & 97 & 78 \\
\hline
\end{tabular}

TABle 3.4: Performance comparison for different datasets on classification accuracy (\%). Bold face indicates best performance.

\begin{tabular}{|c|c|c|c|c|c|c|c|c|}
\hline Dataset & Raw & PCA & Sparse PCA & RP & RP+PCA & mSDA & SRP+PCA & SSRP \\
\hline comp vs rec & 92.7 & 93.2 & 88.3 & $72.1 \pm 2.1$ & $85.7 \pm 1.3$ & 94.0 & $91.6 \pm 0.06$ & $\mathbf{9 4 . 4} \pm 0.13$ \\
comp vs sci & 86.1 & 86.8 & 81.8 & $67.6 \pm 3.0$ & $80.5 \pm 1.7$ & 88.9 & $84.5 \pm 0.08$ & $\mathbf{8 9 . 1} \pm 0.11$ \\
comp vs talk & 94.5 & 94.0 & 89.7 & $74.5 \pm 2.5$ & $88.6 \pm 1.2$ & $\mathbf{9 4 . 9}$ & $92.5 \pm 0.11$ & $\mathbf{9 4 . 9} \pm 0.09$ \\
rec vs sci & 89.2 & 88.9 & 82.2. & $64.5 \pm 2.3$ & $79.3 \pm 0.9$ & 87.0 & $88.9 \pm 0.09$ & $\mathbf{9 0 . 4} \pm 0.13$ \\
rec vs talk & 86.0 & $\mathbf{9 0 . 7}$ & 84.6 & $66.7 \pm 3.8$ & $82.1 \pm 1.9$ & 89.7 & $89.4 \pm 0.08$ & $90.6 \pm 0.10$ \\
sci vs talk & 76.9 & 86.7 & 82.5 & $66.0 \pm 3.1$ & $80.0 \pm 2.3$ & 87.2 & $81.2 \pm 0.12$ & $\mathbf{8 8 . 7} \pm 0.15$ \\
Farm Ads. & 84.8 & 86.0 & 86.3 & $75.4 \pm 2.7$ & $82.7 \pm 1.1$ & 84.3 & $86.7 \pm 0.10$ & $\mathbf{8 8 . 8} \pm 0.19$ \\
\hline average & 87.2 & 89.4 & 85.0 & 69.5 & 82.7 & 89.4 & 87.8 & $\mathbf{9 1 . 0}$ \\
\hline
\end{tabular}

where $n=7$. Under the null hypothesis that the performance difference of the methods pair is zero, the $t$-statistic follows a $t$-distribution with $n-1=6$ degrees of freedom. From the table of the $t$-distribution, the confidence levels that SSRP outerperforms mSDA and SSRP outerperforms PCA are 97.2\% and 99.7\%, respectively.

\subsubsection{Analysis of Number of Layers}

Multi-layers structures have been successful in learning new representation. Therefore, it is meaningful to study the influence of the number of layers of SSRP on classification performance. Here, we set the number of layers as 20. The dimensionality is reduced layer by layer and converged to 100, according to our proposed protocol as shown in Eq. (3.10). Fig. 3.4 shows the trend of changing dimensionality in SSRP. It is clear that the dimension of the output of SSRP can be converged to 100 regardless of the original dimension. To measure the quality of the feature learned by different layers of SSRP, we apply the linear SVM on the corresponding representation for these seven datasets. Fig. 3.5 demonstrates the classification accuracies on these seven datasets as a function of the number of layers in SSRP. 


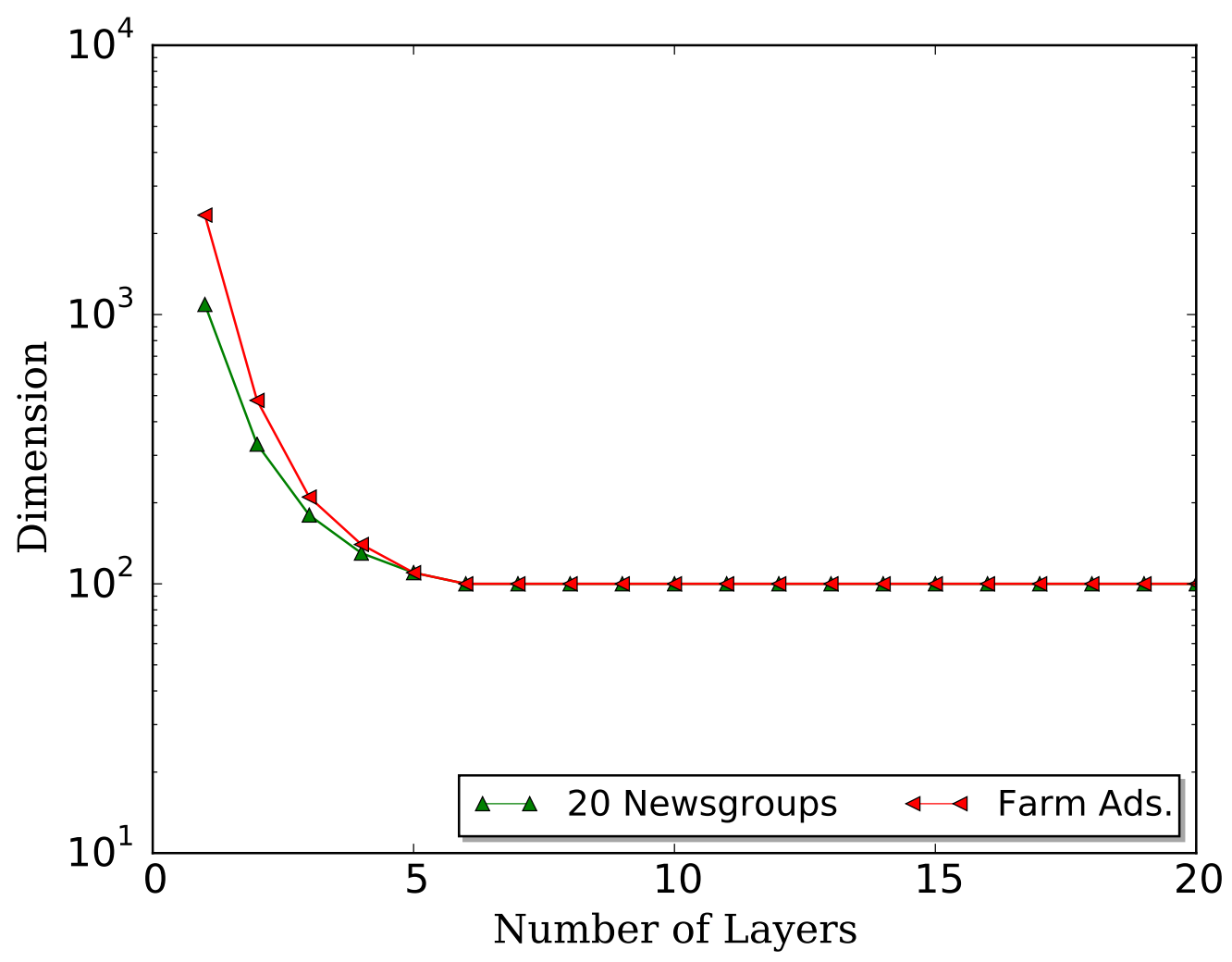

Figure 3.4: Dimensionality on the seven datasets (the first six ones belong to 20Newsgroup and the final one belongs to farm ads) at the output of different layers in SSRP

We can observe that the classification performance improves with the increasing number of layers. The performance improvement is trivial when the number of layers reaches to a certain value. Further, the performance does not deteriorate even when more layers are used. This shows the robustness of SSRP.

\subsection{Conclusion}

In this chapter, we have proposed a novel dimensionality reduction framework based on RP, called Semi-Random Projection. The random subsampling of features makes our approach computationally efficient even for data with a very high dimensionality, while learning scheme is integrated into this SRP framework to improve the discriminative power of the latent space. This SRP framework provides a general platform to derive efficient dimensionality reduction approaches for highdimensional data, in which different learning schemes can be applied. In addition, 


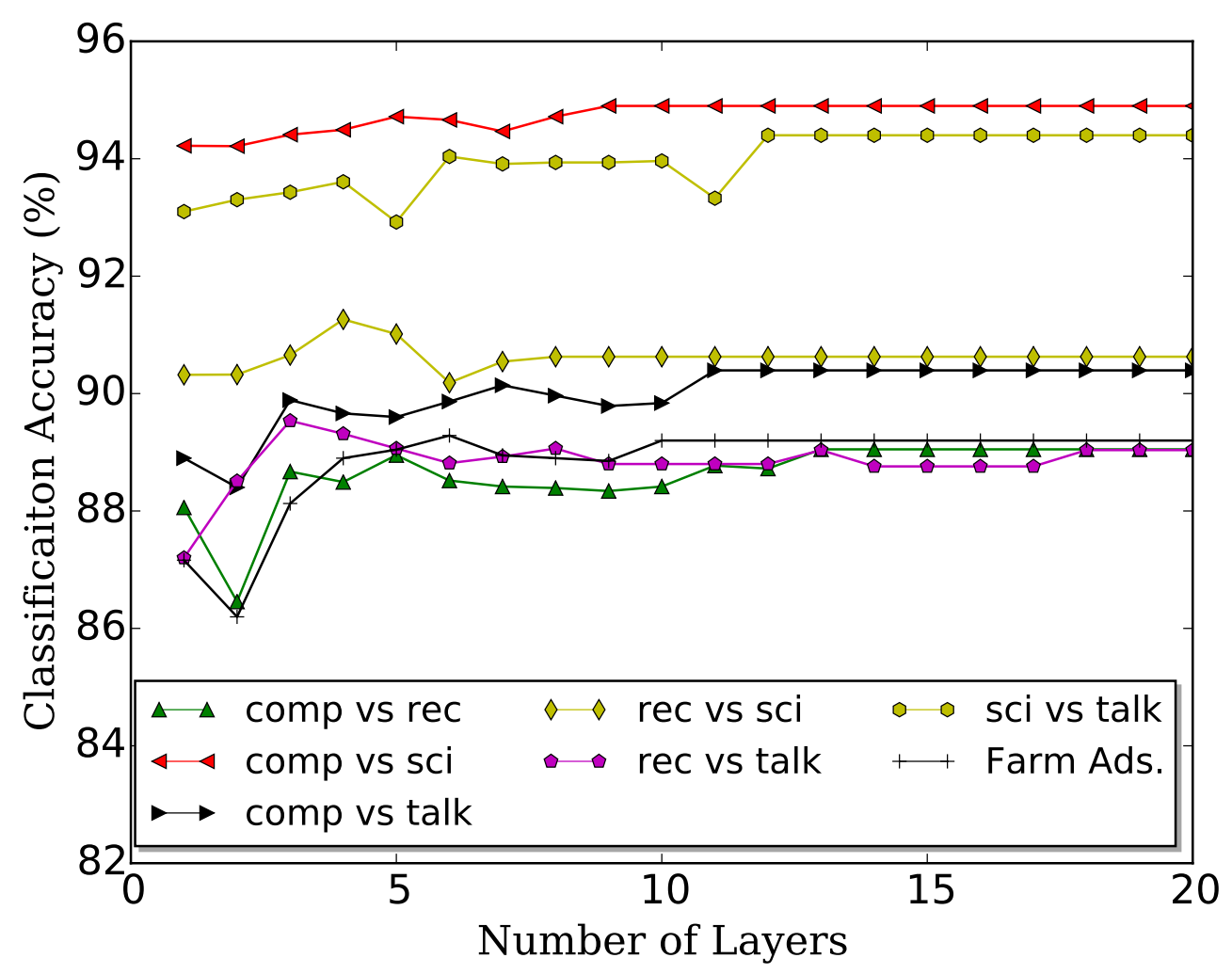

Figure 3.5: Classification accuracies (\%) on the seven datasets at the output of different layers in SSRP

we have proposed to stack SRP modules to implement a deep structure. Compared to other methods relying on deep architectures, our method can be implemented efficiently and the features learning by SSRP are effective.

The next chapter concentrates on another kind of text representation learning methodology: neural compositional models. 


\section{Chapter 4}

\section{Topic-Aware Deep Compositional Models for Sentence Representation}

This chapter is motivated by the analysis of neural compositional models in section 2.3.2 that looks into the usage of the single prototype word embeddings. The adoption of single-sense word embeddings hinders accurate semantic modeling no matter what kinds of neural networks are used unless multi-sense word embeddings are adopted. However, multi-sense word embeddings have their own problems which will be discussed next in section 4.1.3. To alleviate these limitations, we integrate topic models into two state-of-arts deep compositional models: CNN and LSTMs and propose Topic-Aware CNN (TopCNN) and Topic-Aware LSTM (TopLSTM). The topic information inferred from topic models is used to enrich the word-embeddings before composition and the learned sentence-embeddings after composition in the framework of TopCNN and TopLSTMs. Via this structure, the multi-sense of words can be captured in modeling senteces' meaning and an effective representation of sentences can be learned, which have been verified by various text classificaiton tasks.

This chapter is organized as follows. After reviewing our motivations and related works in Section 4.1, we introduce our proposed sentence representation learning models: Topic-Aware Convolutional Neural Network (TopCNN) and Topic-Aware Long Short Term Memory Networks (TopLSTMs) in Section 4.2. In Section 4.3, 
experimental results on several sentence classification tasks are presented and analyzed. Finally, concluding remarks are provided in Section 4.4.

\subsection{Background}

In this section, motivations behind our work and related works on topic models and multi-sense word embeddings will be discussed, respectively.

\subsubsection{Motivations}

Whatever neural compositional model is used, the composition process is performed on the embeddings of each word in the sentence. In most scenarios, these word embeddings are pre-trained word vectors, which can be regarded as general representations since these embeddings are learned from a large corpus (over billions words). When applied to a specific application, these word embeddings can be fine-tuned to improve the performance. Despite the remarkable performance of the compositional models on various sentence modeling tasks, these models ignore the multi-sense nature of words. The majority of deep compositional models take single prototype word embeddings as input, in which each word is associated with a single embedding. However, a word may have multiple senses (for example, bank has two totally different meanings: sloping land and financial institution), and the appropriate sense of a word should be determined by the context of its occurrence. To address these problems, some works have proposed multi-sense embeddings-based models that represent each word by multiple sense-specific embeddings [75, 76, 91-93], and a few pioneering work have attempted to integrate word sense disambiguation into various compositional models for semantic capture [58, 94-97]. In these multi-sense word embeddings based compositional semantic models, two operations are performed before the semantic composition: one is to learn multi-sense embeddings and another is to disambiguate the word sense. These two parts are complex and challenging, and are usually conducted separately.

In this chapter, to address the aforementioned problems, we propose a lightweight and flexible framework for disambiguation in compositional models by introducing topic models. This framework aims to combine the two steps together. Here, 
we focus on two specific forms of composition, i.e., Convolutional Neural Network (CNN)[98], and Long Short Term Memory Network (LSTM)[99]. In recent years, some CNN-based and LSTMs-based sentence modeling works have been reported $[2,28,29,100,101]$. However, these models do not consider the multi-sense issue. Relying on the widely used topic model: Latent Dirichlet allocation (LDA*) [102], the word-topic distribution $p(w \mid z)$ and document-topic distribution $p(z \mid d)$ can be inferred from the co-occurrence of words within the task-specific corpus. A topic $z_{i}$ can thus be allocated to each word $w_{j}$ in a specific document $d_{h}$ according to the probability $p\left(z_{i} \mid w_{j}, d_{h}\right) \propto p\left(w_{j} \mid z_{i}\right) p\left(z_{i} \mid d_{h}\right)$. Since the length of a sentence is limited, the entire sentence can be regarded as an appropriate context for word sense disambiguation so that the probability $p\left(z_{i} \mid w_{j}, d_{h}\right)$ can be used to induce different sense for the same word under different context. Here, the context is defined as the sentence itself. The probability information along with topics embeddings encoded by fixed-length vectors can be integrated at two different levels of CNN and LSTMs, including word-level and sentence-level. Here, details of Topic-Aware CNN (TopCNN) will be elaborated and Topic-Aware LSTMs (TopLSTMs) will be briefly explained.

\subsubsection{Topic Models}

Topic modeling approaches, such as Latent Dirichlet Allocation (LDA ${ }^{*}$ ) and Probability Latent Semantic Analysis (PLSA), are derived from Latent Semantic Analysis, with the goal of reducing high dimensions of the document-term occurrence matrix into low dimensions denoted as latent topics $[7,44,102,103]$. The basic idea behind these methods is that the word choice in a document is influenced by the topic of the document probabilistically. The generation process of each word in a document is considered as a combination of three steps: a distribution over topics for the document is first selected randomly, a topic is then chosen from the above distribution, and finally a word is picked from the corresponding distribution over the vocabulary.

In the present work, $\mathrm{LDA}^{*}$ is employed to obtain word-topic and topic-document probabilities to enrich word embeddings for the subsequent use in the deep compositional model. Different from PLSA, LDA* employs Dirichlet distribution as the prior probability for topic modeling. 


\subsubsection{Multi-sense Word Embeddings for Semantic Compo- sition}

In most word embeddings models, one word is represented by a unique vector without considering the polysemy and homonymy in natural language [17, 19]. The learned embeddings may be the average sense of its multiple senses. To alleviate the problem, some recent works have proposed multi-sense embeddings [75, 76, 9193, 104]. Pre-clustering of the context of each word is often used to determine its specific sense [75, 76]. In [93], topical word embeddings are proposed, in which word embeddings are learned based on words occurrence and their topic information learned from LDA* Some other works rely on external resources, such as Wikipedia and WordNet for word disambiguation [91, 104]. In [92], a threshold value based on a non-parametric model is used to determine the number of senses of each word adaptively based on context pre-clustering. Most of the above works focus on the semantic modeling at word-levels without considering the semantic composition procedure. It should be noted that the topical word embeddings in [93] also employs LDA* for word disambiguation. Different from the above topical word embeddings work that mainly focuses on word-level representation, our work here aims to develop a semantic composition model for sentence representation that considers multi-sense nature of words.

In a recent introductory and exploration work of multi-sense word embeddings based semantic composition [94], the pipelined framework shown in Fig. 4.1 incorporates word disambiguation into semantic composition via the following three steps: 1) multi-sense embeddings learning: each word is represented by multiple sense-specific embeddings; 2) sense induction: for a target language unit (sentence), the sense of each word and the corresponding embeddings are determined; and 3) representation learning based on sense-specific embeddings: compositional models are applied on the sense-specific embeddings. Shallow models including additive, multiplication and tensor operations combined with prior disambiguation are proposed in $[58,96]$, while deep compositional models based on neural networks are adopted in $[94,95,97]$.

Our proposed topic-aware deep compositional framework is different from the above mentioned works in the following aspects. Firstly, our framework is a lightweight and flexible without requiring multi-sense embeddings learning and sense induction 
as separated steps to the final compositional module. Via topic awareness, the embeddings of words used in our model consists of two parts: the general sense learned from a large corpus and the topic-specific sense learned from the task-specific corpus. Therefore, our framework does not require additional multi-sense embeddings models. Secondly, in our proposed framework, the applied LDA* model utilizes the whole co-occurrence statistics of the corpus for word disambiguation instead of the neighbourhood contexts required by previous works $[93,105]$. In these previous works, the hyper-selection of lengths about context is a very tricky issue. Thirdly, our framework is more extensible than these previous works, and various deep compositional models with different neural networks can be developed under this framework. In our work, two state-of-arts compositional models including CNN and LSTMs are adopted as examples to derive two topic-aware compositional models, namely TopCNN and TopLSTMs, respectively.

In the literature, there are some works that incorporate multiple versions of word embeddings into CNN model for sentence classification, where one version of word embeddings can be regarded as one channel [106, 107]. Our proposed TopCNN models utilize word embeddings and topic embeddings, which can also be regarded as two sets of word embeddings. However, the previous works still can not address the multi-sense issues, since word embeddings in each channel are shared by all occurrences of one certain word in corpus. By contrast, topic embeddings in our work are determined by occurrences of words in different context, i.e., the probability information of words. This means that the topic embeddings of word is derived by its context, and is able to derive multi-sense word embeddings. In addition, the topic embeddings in our work can be incorporated into word and sentence levels, while the previous works can only integrate multi-prototype word embeddings at word level.

\subsection{Topic-Aware Deep Compositional Models}

Assuming that word embeddings are pre-trained from a large corpus denoted as a

matrix $\mathbf{W} \in \mathbb{R}^{V \times d}$, where $V$ is the vocabulary size and $d$ is the dimension of word embeddings. $\mathrm{LDA}^{*}$ is applied to the task-specific corpus to derive the probability distributions of words and sentences over latent topics, i.e., $p\left(w_{i} \mid z_{i}\right)$ and $p\left(z_{i} \mid s\right)$, where $z_{i}$ belongs to the pre-defined topic set $Z=\left\{z_{1}, z_{2}, \ldots, z_{T}\right\}$. Here, we adopted 


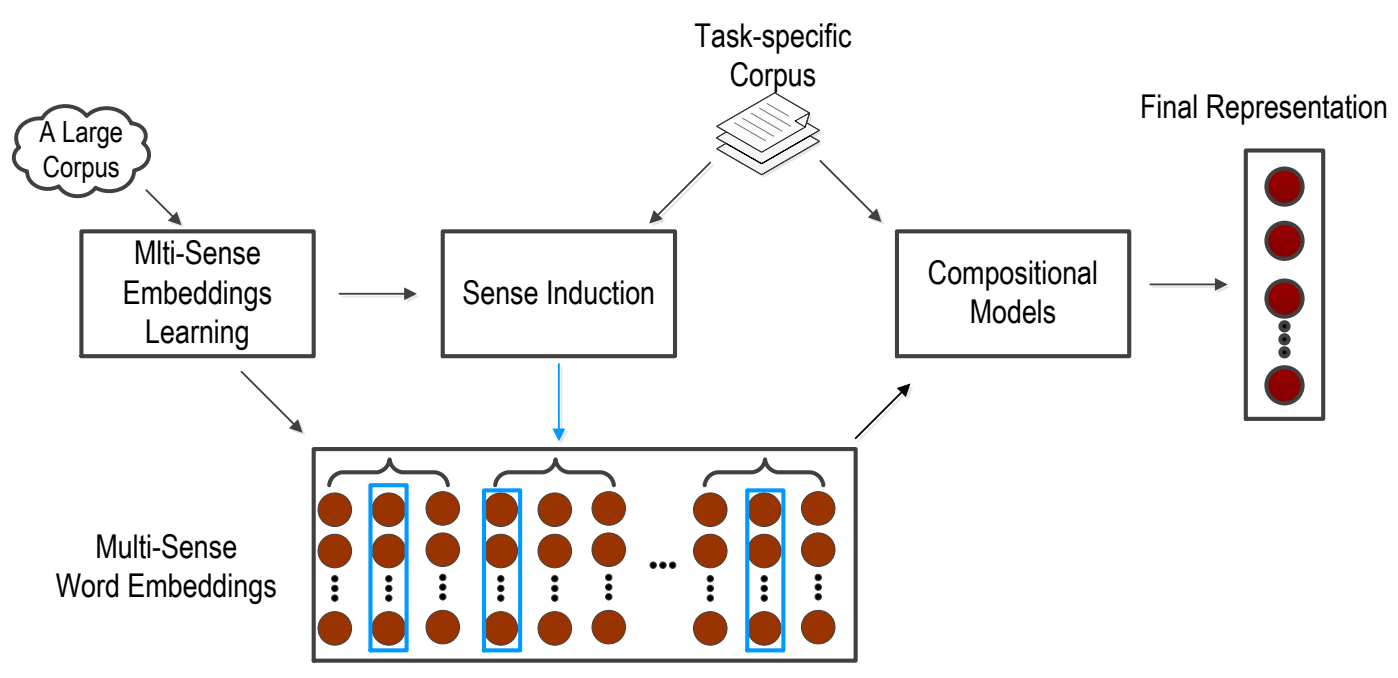

Figure 4.1: Illustration of Semantic Composition Models built on top of MultiSense Embedding.

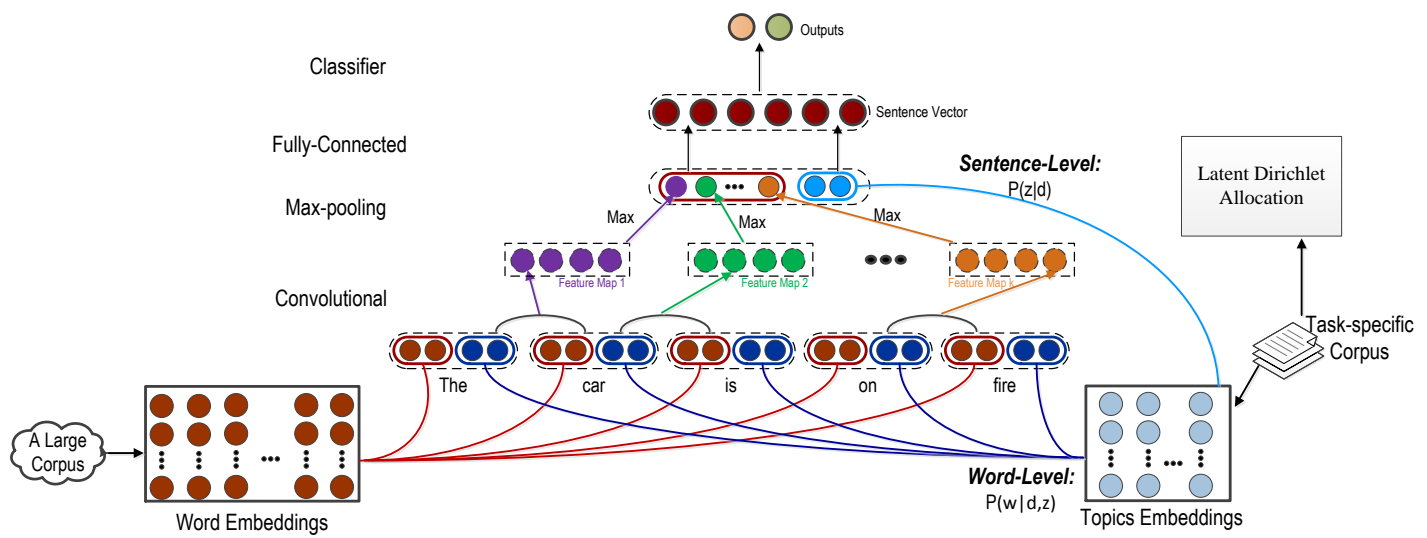

FiguRE 4.2: The Framework of our TopCNN models for sentence representation learning.

a variant of LDA*: Biterm Topic Model [108], which is effective for short text modeling. Embeddings for topics, denoted by matrix $\mathbf{W}_{t} \in \mathbb{R}^{T \times d_{t}}$, will also be learned. For word embeddings, there are various public resources including pretrained word embeddings and tools for implementation. For topic embeddings, we will present four simple but effective schemes to learn representations for each topic after introducing the whole structures of our TopCNN and TopLSTMs. At last, the additional computational burden caused by LDA* will be discussed. 


\subsubsection{Topic-Aware CNN}

The structure of the proposed Topic-Aware Convolutional Neural Network (TopCNN) is shown in Figure 4.2. It illustrates the whole process to learn the sentence vector from the input sentence. Word Embeddings are learned in a large corpus, which is regarded as the general sense of words. Then, with the favor of LDA* trained in the task-specific corpus, the probability information can be utilized at word-level and sentence-level, respectively. At the word-level, the topic-specific embeddings of words are concatenated behind the general word embeddings. At the sentence-level, the topic-specific embeddings of words are concatenated behind the output vector of max-pooling layer. After multiple fully-connected layers, the learned sentence representations are finally fed into the classifier. For a sentence $s=\left(w_{1}, w_{2}, \ldots, w_{l}\right)$ with $l$ words, the TopCNN aims to learn its embeddings as follows:

Word-Level : For word $w_{i}$ in sentence $s$, its probability over the latent topics $p\left(z_{i} \mid w_{j}, s\right) \propto p\left(w_{j} \mid z_{i}\right) p\left(z_{i} \mid s\right)$ is calculated. After normalization, the topical distribution of word $w_{i}$ is denoted by vector $\mathbf{p}_{w_{i}}$ :

$$
\begin{array}{r}
\mathbf{p}_{w_{i}}=\left[p\left(z_{1} \mid w_{i}, s\right), p\left(z_{2} \mid w_{i}, s\right), \ldots, p\left(z_{T} \mid w_{i}, s\right)\right] \\
\text { s.t } p\left(z_{i} \mid w_{i}, s\right) \geq 0 \text { and }\left|\mathbf{p}_{w_{i}}\right|=1
\end{array}
$$

where the $i$-th element in $\mathbf{p}_{w_{i}}$ denotes the probability that word $w_{i}$ is assigned to the $i$-th topic. This topical distribution $\mathbf{p}_{w_{i}}$ is a useful resource for word disambiguation, which represents each word occurred in a specific context by the corresponding topic distribution. The topical information is derived by applying LDA* to the word-document occurrence statistics instead of the word and its window-based context. To utilize the topical information of each word in the compositional model, the topic-specific embeddings for each word $w_{i}$ based on the topical information $\mathbf{p}_{w_{i}}$ and the embeddings for topics $\mathbf{W}_{t}$ is derived as follows:

$$
\mathbf{t}_{w_{i}}=\sum_{i=1}^{T} p\left(z_{i} \mid w_{i}, s\right) * \mathbf{W}_{t}[i]=\mathbf{p}_{w_{i}} \times \mathbf{W}_{t}
$$

The general word embeddings pre-trained over a large corpus is concatenated with the topic-specific embeddings to form the representation of the word as $\mathbf{x}_{\mathbf{i}} \in \mathbb{R}^{d+d_{t}}$ :

$$
\mathbf{x}_{i}=\mathbf{W}\left[w_{i}\right] \oplus \mathbf{t}_{w_{i}}=\left[\mathbf{W}\left[w_{i}\right] ; \mathbf{t}_{w_{i}}\right]
$$


where $\oplus$ denotes concatenation operation. As shown in Eq. 4.3, the final representation of a word consists of two parts: one is the general word embeddings and another is the topic-specific embeddings. General word embeddings provide the general sense of the word, while the topic-specific embeddings are able to disambiguate the senses of the words.

Convolution: the convolution operation is defined as a multiplication operation between a filter vector $\mathbf{u} \in \mathbb{R}^{m\left(d+d_{t}\right)}$ and an concatenation vector representation $\mathbf{x}_{i: i+m-1}$ given by:

$$
\mathbf{x}_{i: i+m-1}=\mathbf{x}_{i} \oplus \mathbf{x}_{i+1} \oplus \cdots \oplus \mathbf{x}_{i+m-1}
$$

where $\mathbf{x}_{i: i+m-1}$ represents a window of $m$ words starting from the word indexed as $i$. A bias term $b$ is added to the output of the convolution operation, and thus the final operation is given as:

$$
c_{i}=g\left(\mathbf{u}^{T} \mathbf{x}_{i: i+m-1}+b\right)
$$

where $*^{T}$ denotes the transpose of a matrix $*$ and $g$ is a non-linear activation function such as Sigmoid, Hyperbolic Tangent, or ReLu. In our work, ReLU is adopted due to its reported good performance [109], but other activation functions are equally applicable.

Each vector $\mathbf{u}$ can be regarded as a filter. The scalar value $c_{i}$ can be regarded as the learned feature in the $i$-th window of words under filter $\mathbf{u}$. The width of the filter, i.e., window size $m$, can be varied. The setting of window size will be discussed in the experiment section 4.3.2.

Max-pooling: The convolution operation over the whole sequence of words is applied by sliding the window from the beginning to the ending of the sentence. Since each sentence of length $l$ has multi-windows of words $\left\{\mathbf{x}_{1: m}, \mathbf{x}_{2: m+1}, \ldots, \mathbf{x}_{l-m+1: l}\right\}$, a feature map can be obtained and the dimension of the feature map is determined by the length of the sentence $l$ and the window size $m$ :

$$
\mathbf{c}_{j}=\left[c_{1}, c_{2}, \ldots, c_{l-m+1}\right]
$$

where the index $j$ denotes the $j$-th filter. By conducting the max-pooling operation in the feature map obtained in the convolutional layer, the single feature corresponding to the filter is obtained as: 


$$
h_{j}=\max \left(\mathbf{c}_{j}\right)
$$

where $h_{j}$ corresponds to the $j$-th dimension of the output of the pooling layer. Generally, multiple filters are applied with different weights and different window sizes to derive a feature vector. The motivation behind the max-pooling operation is to filter out meaningless combinations of words. Another effect of max-pooling is that it produces a fixed-length feature vector regardless the sentence lengths.

Sentence-Level: Considering various semantic and syntactic patterns in natural language, we design multiple filters with different window sizes and randomly initialized weights to match and capture these patterns. After convolution and max-pooling operations, the produced scalar is the output of a filter applied on the entire sentence. Assuming there are $k$ filters, the output of the pooling layer is the concatenation of all outputs of the filters:

$$
\mathbf{h}=\left[h_{1}, h_{2}, \ldots, h_{k}\right]
$$

$\mathbf{h}$ can be used as the final representation of the input sentence, if the different filters are enough to discover all meaningful structure patterns of the sentence. However, $\mathbf{h}$ may not convey the complete semantic meaning of the entire sentence, since each element in $\mathbf{h}$ is only the activation of a local window of words. Although the window size of applied filters can be set to the length of the sentence, the high computational cost and the sparsity problem are the inherent challenges. To address the problem, we propose to enrich the representation based on topical information similar to the integration of topic model at word-level. After applying LDA $^{*}$, the probabilities of the sentence over each topic is given by:

$$
\begin{aligned}
\mathbf{p}_{s}= & {\left[p\left(z_{1} \mid s\right), p\left(z_{2} \mid s\right), \ldots, p\left(z_{T} \mid s\right)\right] } \\
& \text { s.t } p\left(z_{i} \mid s\right) \geq 0 \text { and }\left|\mathbf{p}_{s}\right|=1
\end{aligned}
$$

The sentence's probabilities are then transformed into an embedding based on embeddings for topics, which is given by:

$$
\mathbf{t}_{s}=\sum_{i=1}^{T} p\left(z_{i} \mid s\right) * \mathbf{W}_{t}[i]=\mathbf{p}_{s} \times \mathbf{W}_{t}
$$


where $\mathbf{t}_{s} \in \mathbb{R}^{d_{t}}$ is the topic-specific embedding for the sentence. Different from $\mathbf{h}$, which is composed of the activations of the local windows of words over the sentence, $\mathbf{t}_{s}$ is the activation of the global context of the sentence. Therefore, the two embeddings can be concatenated to represent the sentence as:

$$
\mathbf{s}=\mathbf{h} \oplus \mathbf{t}_{s}=\left[\mathbf{h} ; \mathbf{t}_{s}\right]
$$

Fully-Connected Layers and Classifier: The learned representation of the sentence is passed to multiple hidden layers to seek a higher-level sentence representation. Several fully-connected dense layers are stacked together, in which the output of one layer is used as the input of the next layer. The computation in each layer is given by:

$$
\mathbf{o}_{i}=g\left(\mathbf{B}_{i} \mathbf{q}_{i}+\mathbf{b}_{i}\right)
$$

where $\mathbf{o}_{i}$ and $\mathbf{q}_{i}$ denote the output and input of the $i$-th fully-connected layer, respectively. $\mathbf{B}_{i}$ and $\mathbf{b}_{i}$ represent the transformation matrix and the bias term in the $i$-th fully-connected layer, respectively. The function $g$ is also set to the rectified linear unit (ReLU). It is clear that the input of the first fully-connected layer is the initial representation of sentence, i.e., $\mathbf{q}_{0}=\mathbf{s}$. The output of the last layer: $\mathbf{o}_{r-1}$ is regarded as the final representation of the input sentence, assuming $r$ fully-connected dense layers are successively stacked.

The final learned representation of the input sentence is fed into the classification layer. Here, we adopt a fully connected softmax layer to predict the probability distributions of the sentence over the whole label set.

Training and Regularization: Given the predicted outputs and true labels, the cross-entropy errors over training corpus can be calculated and back-propagated to update model parameters. The model parameters include filter weights, parameters of fully-connected hidden layers and softmax classification layer. In addition, word embeddings $\mathbf{W}$ and topic embeddings $\mathbf{W}_{t}$ are also fine-tuned. To avoid overfitting, regularization technique is applied. Here, we follow some settings of the CNN structure proposed in [28]. Firstly, dropout noise with masking probability $p$ is applied on the feature vector fed into the fully-connected softmax layer [110]. Then, a $l 2$-norm constraint over the filter weights $\mathbf{u}$ and fully-connected hidden layers' 


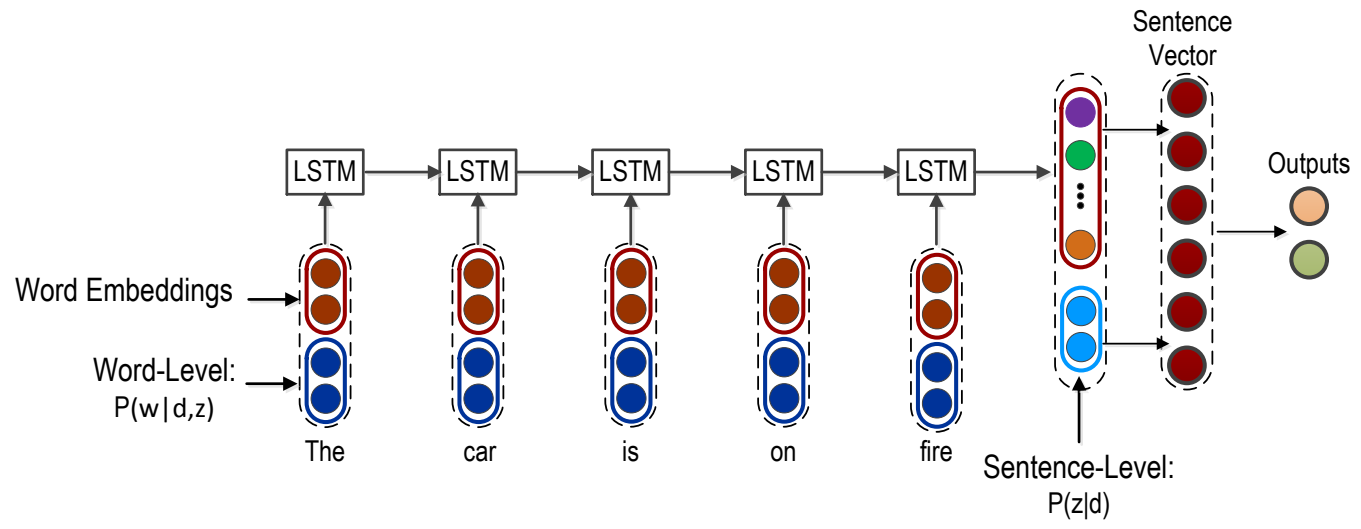

Figure 4.3: The Framework of our TopLSTMs for sentence representation learning.

transformation matrix $\mathbf{B}_{i}$ is imposed during training. The whole network is trained by stochastic gradient descent with Adadelta update rule that has been shown as an effective and efficient backpropagation algorithm [111]. The training corpus are shuffled randomly to generate a stream of mini-batches for model training.

\subsubsection{Topic-Aware LSTM}

The structure of our proposed Topic-Aware Long Short Term Memory Networks (TopLSTMs) is shown in Figure 4.3. Details of LSTM can be found in section 2.3.2. Similar to TopCNN, topic embeddings of words are incorporated into the corresponding word embeddings, and the concatenation of these general and topicspecific word embeddings is fed into LSTM units. As shown in Eq. (2.14), $\mathbf{U}\left[x_{t}\right]$ is set to be the concatenation of general word embeddings and topic-speicific embeddings. The topic embeddings of sentence can also be concatenated with the outputs of the proceeding step. The learned representation for the sentence is finally fed into multiple hidden layers and the final classification layer.

\subsubsection{Embeddings Learning for Topics}

In the above sections, the probability information derived by $\mathrm{LDA}^{*}$ in the form of embeddings for topics $\mathbf{W}_{t}$ is incorporated into the convolutional semantic composition process. In the following, we present four models of topic embeddings learning including random, additive, topical neural models and joint neural models. 
Random Model: These topic embeddings are first randomly initialized, and are then fine-tuned during the training stage.

Additive Model: The motivation behind the additive model is very intuitive that the topic can be represented by a list of top words ranked by probabilities belonging to this topic. Therefore, the meaning of the topic can be approximated by the meanings of these top words $\mathbf{W}_{t}$. We average the embeddings of these top-ranked words to obtain the embeddings of the topic:

$$
\mathbf{W}_{t}[i]=\frac{1}{h} \sum_{j=0}^{h-1} \mathbf{W}\left[w_{j}\right]
$$

where $\mathbf{W}_{t}$ denote the embeddings for the $t$-th topic, $w_{j}$ is one of the top $h$ words whose probabilities belonging to topic are the largest, and $\mathbf{W}$ is the pre-trained word embeddings matrix. It is clear that under the additive model, the embeddings for topics and words have the same dimensionality.

Topical Neural Model: The neural model is inspired by the efficient word embedding framework word2vec, including Continuous Bag-of-Word (CBoW) model and Skip-Gram [19]. The training objective of $\mathrm{CBoW}$ is to predict the target word by combining the embeddings of context words, while the objective of Skip-Gram is to predict the context word by the target and central word in the context. In topic embedding learning, the word occurred in documents can be replaced by the corresponding topics. Based on LDA*, we firstly assign the topic to each word $w_{i}$ based on Eq. 4.1, from which the topic having the highest probability is picked up. The assigned topic is then regarded as a pseudo word to replace the actual word. Thus, the idea of word embedding learning can naturally be used to learn topic embeddings. Similar to word2vec, the maximization objectives of our neural model-based topic embedding learning are defined as follows:

$$
\begin{gathered}
L(s)_{\text {cbow }}^{T N M}=\frac{1}{M} \sum_{i=1}^{M} \log \left(\mathbf{z}_{i} \mid \mathbf{z}_{\text {context }}\right) \\
L(s)_{\text {skip }}^{T N M}=\frac{1}{M} \sum_{i=1}^{M} \sum_{-k \leq j \leq k} \log \left(\mathbf{z}_{i+j} \mid \mathbf{z}_{i}\right)
\end{gathered}
$$


where $\mathbf{z}_{i}$ denotes the embedding for the topic assigned to the $i$-th word in the sentence $s$. In Figure 4.4(a), the architecture of topical neural model is shown.

Joint Neural Model: One concern behind the above topical neural model lies in the possible large divergence between the learned topic embeddings and the word embeddings. Since topical neural model regards each topic as a pseudo word, the topic embeddings are learned without word information. To address this issue, a joint neural model is proposed by taking each word-topic pair instead of topic as a pseudo word. Similar to Eqs. 4.14 and 4.15, the target functions of joint neural model are defined as:

$$
\begin{gathered}
L(s)_{\text {bow }}^{J N M}=\frac{1}{M} \sum_{i=1}^{M} \log \left(\left\{\mathbf{w}_{i}, \mathbf{z}_{i}\right\} \mid\left[\mathbf{w}_{\text {context }}, \mathbf{z}_{\text {context }}\right]\right) \\
L(s)_{\text {skip }}^{J N M}=\frac{1}{M} \sum_{i=1}^{M} \sum_{-k \leq j \leq k} \log \left(\left\{\mathbf{w}_{i+j}, \mathbf{z}_{i+j}\right\} \mid\left\{\mathbf{w}_{i}, \mathbf{z}_{i}\right\}\right)
\end{gathered}
$$

For each word topic pair $\left\{\mathbf{w}_{i}, \mathbf{z}_{i}\right\}$, the word embeddings and topic embeddings are concatenated together. Compared to the topical neural model, the joint neural model may encounter sparsity problem. However, in this model, the word embeddings are pre-trained and fixed during the training, while the topic embeddings are fine-tuned in the local corpus. The advantages of this settings are two-folders: one is the alleviation of the sparsity issue during model training and another is the reduction of the domain difference between learned word embeddings and topic embeddings. It should be noted that the proposed Joint Neural Model is similar to the TWE-3 model proposed in [93]. However, our model uses word2vec embeddings trained on a corpus with billions of words, while TWE-3 learns word and topics embeddings from task-specific corpus. The joint neural model is illustrated in Figure 4.4(b).

Among the four topic embeddings learning methods including random, additive, topical neural and joint neural models, the first two methods belong to lazy methods without any learning schemes, while the last two are based on the word2vec model. The difference between the topical neural model and the joint neural model is that the joint neural model considers word and topic information jointly in the training process, while the topical neural model relies on topic information only. A detailed experimental analysis over the four methods is given in section 4.3.5. 


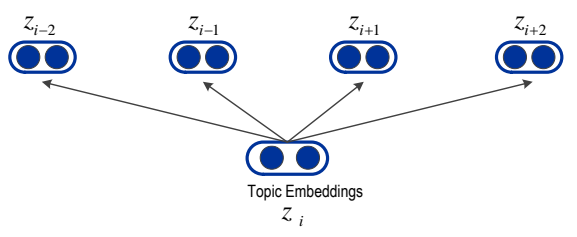

(a)

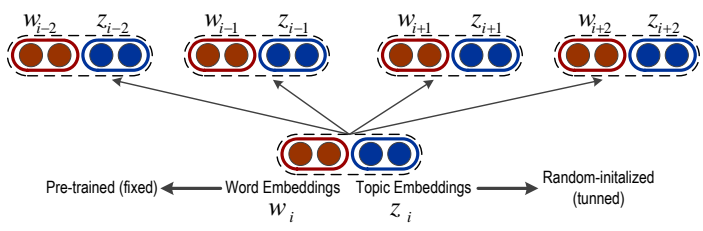

(b)

FiguRE 4.4: Two neural network architectures (Skip-gram based) for topic embeddings learning: (a) Topical Neural Model; (b) Joint Neural Model.

\subsubsection{Complexity Analysis}

The proposed topic-aware deep compositional framework is a pipelined system built upon LDA $^{*}$ and deep compositional models. Compared to the basic deep composition models CNN or LSTMs, the complexity is increased due to the use of LDA* In this study, we use a LDA* variant named biterm topic model (BTM) to model topics in short texts [108]. The computational complexity of the Gibbs sampling implementation of BTM, i.e., the additional computational burden of TopCNN, is $\mathcal{O}\left(K D \frac{\bar{l}(\bar{l}-1)}{2}\right)$, where $K, D$ and $\bar{l}$ denote the topic number, the document number and the average length of document, respectively. Since deep compositional models including CNN and LSTMs usually have huge numbers of parameters to learn, the increased computational complexity due to topic models is negligible.

\subsection{Experiments}

In this section, we evaluate our proposed TopCNN and TopLSTMs on sentence classification tasks. Five benchmark real datasets are used. Several state-of-arts methods including traditional classification methods and sentence models are compared and the sensitivity of our models to the number of topics in LDA* is analyzed. Finally, the topic embedding learning methods and their effects on the performance of our proposed topic-aware deep compositional models are discussed.

\subsubsection{Experimental Settings}

Datasets: Five benchmark datasets are used in the experiment. The datasets and their tasks are described below: 
- MR: Movie Review dataset [112] ${ }^{1}$. Each sample is a movie review sentence. The binary sentiment polarity is defined as positive or negative. The task is to classify each review by its binary sentiment polarity.

- Subj: Subjectivity dataset $[113]^{2}$. Each sample is a sentence. The task is to classify each snippet into subjective or objective categories.

- CR: Customer Reviews dataset $[114]^{3}$. The corpus consists of customer reviews of several products from Amazon. The task is to classify each customer review by its binary sentiment polarity: positive or negative.

- MPQA: Opinion on a phrase level polarity detection subtask of the MPQA dataset $[115]^{4}$. The task is to judge each phrase as positive or negative.

- TREC: Question classification dataset containing 6 different types of classification in $[116]^{5}$. The task is to classify the question content by their question type.

Statistics of these datasets are given in Table 4.1. Since the first four datasets do not have explicit training/testing split, 10-folder cross validation (CV) is applied on them. TREC dataset provides the training/testing split, in which training data have 5452 instances and testing data have 500 instances.

Compared Approaches: Several state-of-arts text classification and sentence modeling methods are compared here. The details of the compared approaches are given below:

* NBSVM and MNB: Naive Bayes SVM and Multinomial Naive Bayes with unigram and bigram features [117].

* G-Dropout and F-Dropout: Gaussian Dropout and Fast Dropout [118]

* RAE and MV-RNN: RAE is Recursive Autoencoders on word embeddings trained from Wikipedia [24]. The composition path is defined by a binary parse tree. MV-RNN is Matrix-Vector Recursive Neural Network with a parse tree [59].

\footnotetext{
${ }^{1}$ https://www.cs.cornell.edu/people/pabo/movie-review-data/

${ }^{2}$ https://www.cs.cornell.edu/people/pabo/movie-review-data/

${ }^{3}$ http://www.cs.uic.edu/iub/FBS/sentiment-analysis.html

${ }^{4}$ http://mpqa.cs.pitt.edu/

${ }^{5}$ http://cogcomp.cs.illinois.edu/Data/QA/QC/
} 
* AdaSent: AdaSent is a self-adaptive hierarchical sentence model [2]. Adasent adopts a hierarchy structure, where consecutive levels are connected through gated recursive composition of adjacent segments, and feeds the hierarchy as a multi-scale representation through a gating network to a particular learning task.

* CNN, $\mathbf{C N N}_{600}$, DCNN and DSCNN: CNN is Convolutional Neural Network on pre-trained word embeddings from word2vec, Google News [28]. Word embeddings, whose dimensionality is 300, are also fine-tuned to learn task-specific embeddings and parallel CNN with the static word embeddings. $\mathrm{CNN}_{600}$ is the same as CNN except that the dimensionality of adopted word embeddings is 600. DCNN is Dynamic Convlolutioanl Neural Network that adopts the dynamic $k$-max pooling instead of max-pooling [29]. DSCNN is dependency-sensitivity CNN, in which CNN is adopted on top of LSTM in a multi-channel [61].

* P.V.: P.V. is Paragraph Vector that learns distributed representations of words and short-text jointly [1]. We feed the learned paragraph vectors into linear SVM for classification and the implementation of P.V. is based on gensim $^{6}$.

* RNN, LSTM and GRUs all belong to recurrent models [68, 72, 99]. Here, we use the library keras to implement these methods. The hidden layer sizes are all set to be same as the embedding size. The same word embeddings adopted by CNN are used for these three recurrent models.

We implemented CNN, $\mathrm{CNN}_{600}$, RNN, P.V, LSTM and GRUs models based on their public sources or packages. For word embeddings in these models, the public word vectors trained on part of Google News dataset by word2vec were adopted here $^{7}$. The dimension of word embeddings is 300. The embeddings of the outof-vocabulary words were randomly initialized. During model training, these pretrained word embeddings are further fine-tuned for a fair comparison. For the rest of the models, their published results in their original papers are cited directly.

\footnotetext{
${ }^{6}$ https://radimrehurek.com/gensim/models/doc2vec.html

${ }^{7}$ https://code.google.com/p/word2vec/
} 
TABLE 4.1: Statistics for the five used datasets in our chapter. $N$ and $v$ count the number of instances and the vocabulary size, respectively. $\bar{l}$ denotes the average length of each dataset. We use 10-fold cross-validation for these datasets without explicit splitting including MR, Subj, CR and MPQA. And for each dataset, we also provide the number of classes denoted as $|\mathbb{C}|$.

\begin{tabular}{clllll}
\hline Dataset & $N$ & $v$ & $\bar{l}$ & $|\mathbb{C}|$ & train/test \\
\hline MR & 10662 & 18765 & 20 & 2 & $\mathrm{CV}$ \\
Subj & 10000 & 21323 & 23 & 2 & $\mathrm{CV}$ \\
$\mathrm{CR}$ & 3775 & 5340 & 19 & 2 & $\mathrm{CV}$ \\
MPQA & 10606 & 6246 & 3 & 2 & $\mathrm{CV}$ \\
TREC & 5952 & 9592 & 14 & 6 & $5452 / 500$ \\
\hline
\end{tabular}

\subsubsection{Specifications for TopCNN}

Firstly, four variants of our proposed TopCNN are considered.

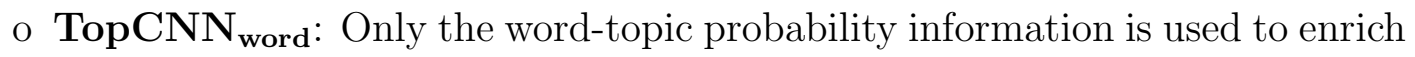
the word embeddings.

o TopCNN $\mathbf{N e n}_{\text {sen }}$ Only the sentence-topic probability information is used to enrich the representation output of the pooling layer.

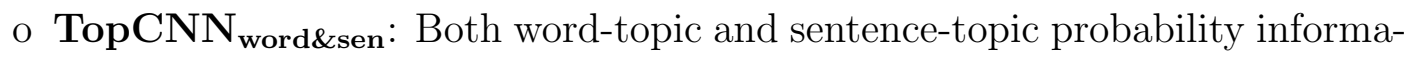
tion are used.

o TopCNN $\mathbf{C n s}_{\text {ens }}$ An ensemble model of the above three variants of TopCNN models by averaging the class probability scores generated by the above three models together.

We used the same pre-trained Google word embeddings in our models and the compared models. For topic model, we used Biterm Topic Model ${ }^{8}$ with a topic number of 100 , i.e., $T=100$. For embeddings of topics, we used the joint neural model, in which the window size is 2 , and the topic embeddings have the same dimension as word embeddings, i.e., $d_{t}=d=300$. For the convolutional layer, a broad range of filter windows sizes $(2,3,4,5,6,7)$ were used, in which each window size corresponds to 200 feature maps. Therefore, we totally have $k=1200$ filters. For the fully-connected hidden layers, the number of layers are set to 2, and the

\footnotetext{
${ }^{8}$ The code for BTM has been kindly provided at https://github.com/xiaohuiyan/BTM
} 
output dimensions of these two layers are set to 1200 and 300, respectively. The other parameters including L2-norm constraint over model parameters, the dropout rate and the training batch size were all set using the validation datasets, which are held-out portions of the training data. Finally, the whole training data were used to train the models with these determined hyperparameters.

Similarly, four variants of our proposed TopLSTMs are considered.

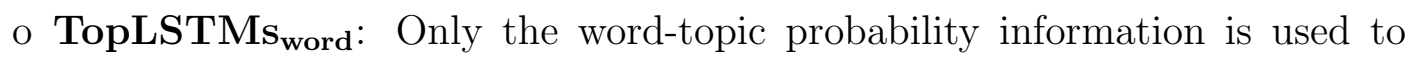
enrich the word embeddings.

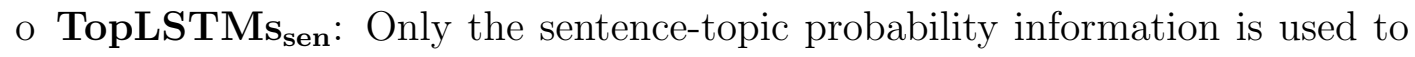
enrich the representation output of the pooling layer.

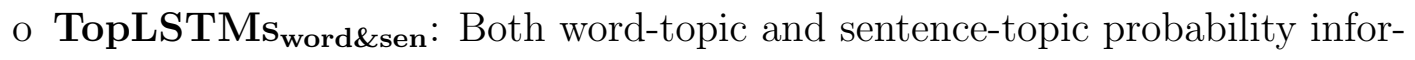
mation are used.

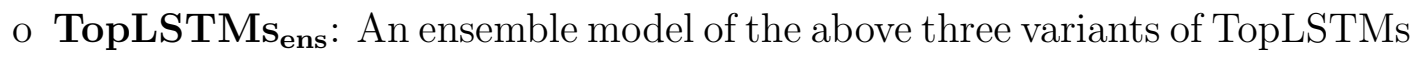
models by averaging the class probability scores generated by the three variants together.

\subsubsection{Experimental Results}

The results are shown in Table 4.2. We compare our proposed methods with several state-of-arts methods including text classification models, CNN-based and RNNbased compositional models. The first observation is that our ensemble model

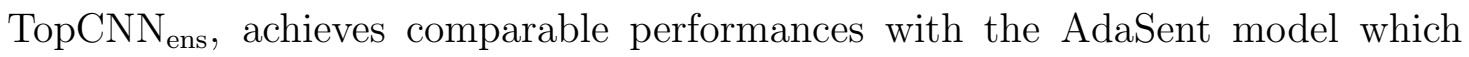
produces up-to-date benchmark results on the five datasets ${ }^{9}$. It can be observed that TopCNN $\mathrm{N}_{\text {ens }}$ outperforms AdaSent in two datasets including $C R$ and TREC. Compared with traditional text classification models including NBSVM, MNB, GDropout and F-Dropout, our proposed TopCNN and TopLSTMs models are able to offer better performance without requiring feature engineering on all datasets. This means that the deep compositional model can indeed boost the representation learning for sentences. As shown in Table 4.2, P.V. model is not able to achieve

\footnotetext{
${ }^{9}$ DTCNN gives the best performance as 95.6. However, it relies on the quality of the parsing operation. As reported in [100], the adopted parser is trained on the annotated TREC dataset so that a high-quality of parsing structure can be guaranteed in this corpus.
} 
satisfying performance over five datasets. This is probably because P.V. is not good at modeling embeddings for short documents due to limited context information.

In experiments, we also compared our methods with other deep compositional models including CNN-based and RNN-based compositional models. Our proposed TopCNN models, especially TopCNN word\&sen $_{\text {and TopCNN }}$ ens, consistently outperform these models on five datasets. Compared to the original CNN model, the achieved performance improvements verify the effectiveness of topical embeddings. In addition, $\mathrm{CNN}_{600}$ performs worse than $\mathrm{CNN}$ on these five datasets, which means the performance improvement of our proposed TopCNN is not resulted from the increased dimensionality of word embeddings. Although the TopLSTMs models do not consistently outperform CNN models, they outperform their basic model: LSTM model. The performance gains achieved by TopCNN ${ }_{\text {ens }}$ and TopLSTMs $\mathrm{ens}_{\mathrm{s}}$ over the basic CNN and LSTM models on the five datasets are shown in Figure 4.5. The significant performance gains of our models show that the integration of LDA* into the deep compositional process can lead to a performance boost. The composition process in our proposed models combine not only general sense of words, but also topic-specific sense of words. In addition, at the sentence level, the output of local receptive fields can be combined with the activation of the whole scope of sentences provided by topic-document probability information. The ensemble of three different levels of topic awareness further improves the model performance.

As shown in Table 4.2, the ensemble version of Topic-aware model performs better than other variants. Ensemble methods always outperform the constituent learning algorithms. Experimental results also show that among the rest three variants of TopCNN and TopLSTMs models, the wordEsen-level model perform best and the sentence-level model perform worst in all datasets. The reason behind the deteriorated performance of the sentence-level model may be that a direct combination of the embeddings learned by basic compositional models and the topical embeddings for sentences is inappropriate due to the discrepancy between the two semantic spaces. In other two variants: the word-level and word\&sen-level models, the convolutional operation is conducted on the combination of general-sense and topic-specific embeddings. The filter weights are learned during training so that the possible semantic space discrepancy may be reduced or even eliminated at the output of the filter. Compared to the word-level model, our wordEsenlevel model further integrates the sentence-topic probability to enrich the output 
TABle 4.2: Classification Accuracies (\%) for Compared Methods on the Whole Five Adopted Datasets. Bold Face Indicates Highest Accuracies

\begin{tabular}{|c|c|c|c|c|c|}
\hline Methods & MR & Subj & $\mathrm{CR}$ & MPQA & TREC \\
\hline NBSVM & 79.4 & 93.2 & 81.8 & 86.3 & - \\
\hline MNB & 79.0 & 93.6 & 80.0 & 86.3 & - \\
\hline G-Dropout & 79.0 & 93.4 & 82.1 & 86.1 & - \\
\hline F-Dropout & 79.1 & 93.6 & 81.9 & 86.3 & - \\
\hline $\mathrm{CNN}$ & 81.3 & 93.5 & 83.9 & 89.4 & 93.0 \\
\hline $\mathrm{CNN}_{600}$ & 79.3 & 92.0 & 81.6 & 87.5 & 91.9 \\
\hline DCNN & - & - & - & - & 93.0 \\
\hline DSCNN & 82.2 & 93.2 & - & - & 95.6 \\
\hline P.V. & 75.9 & 92.2 & 77.9 & 75.4 & 91.5 \\
\hline RAE & 77.7 & - & - & 86.4 & - \\
\hline MV-RNN & 79.9 & - & - & - & - \\
\hline $\mathrm{RNN}$ & 77.2 & 90.9 & 71.8 & 88.6 & 83.8 \\
\hline LSTM & 79.5 & 93.3 & 80.4 & 88.8 & 89.4 \\
\hline GRUs & 80.5 & 93.5 & 82.1 & 89.0 & 91.8 \\
\hline AdaSent & 83.1 & 95.5 & 86.3 & 93.3 & 91.8 \\
\hline$\overline{\text { TopCNN }}_{\text {word }}$ & 81.7 & 93.4 & 84.9 & 89.9 & 92.5 \\
\hline TopCNN $_{\text {sen }}$ & 81.3 & 93.4 & 84.8 & 90.3 & 92.0 \\
\hline TopCNN $_{\text {word\&sen }}$ & 82.3 & 94.3 & 85.6 & 91.1 & 93.6 \\
\hline $\mathrm{TopCNN}_{\mathrm{ens}}$ & 83.0 & 95.0 & 86.4 & 91.8 & 94.1 \\
\hline TopLSTMs $_{\text {word }}$ & 81.2 & 94.1 & 82.6 & 89.6 & 91.5 \\
\hline TopLSTMs $_{\text {sen }}$ & 80.6 & 93.7 & 81.6 & 89.1 & 90.5 \\
\hline TopLSTMs $_{\text {word\&sen }}$ & 80.8 & 94.0 & 82.3 & 89.5 & 91.4 \\
\hline TopLSTMs ens & 81.9 & 94.5 & 82.9 & 90.8 & 91.9 \\
\hline
\end{tabular}

of max-pooling layer or the final time step and feed them into multiple fullyconnected layers before the final classification. The further utilization of topic sen $_{\text {. }}$ probability may explain the better performance of the wordEsen-level model than the word-level model .

Model variances of several comparable models are further investigated, which can reduce the possible bias caused by the randomness of parameters initialization. We conducted testing of these models including P.V., RNN, LSTM, GRUs, CNN and our proposed TopCNN and TopLSTMs models ten times and reported their mean accuracies and corresponding standard deviation in Table 4.3. The model variances of AdaSent is from [2]. As shown in Table 4.3, our proposed TopCNN $\mathrm{Nord}_{\text {wsen }}$ and TopCNN $\mathrm{ens}_{\text {s }}$ achieve the best performance among these methods. 
TABle 4.3: Model Variances for Compared Methods based on Our Own Implementations on the Whole Five Adopted Datasets.

\begin{tabular}{c|c|c|c|c|c}
\hline Methods & MR & Subj & CR & MPQA & TREC \\
\hline P.V. & $73.1 \pm 0.6$ & $90.5 \pm 0.9$ & $76.1 \pm 0.4$ & $72.2 \pm 0.8$ & $89.9 \pm 0.9$ \\
RNN & $77.2 \pm 0.3$ & $89.9 \pm 0.5$ & $71.9 \pm 0.5$ & $88.2 \pm 0.1$ & $83.6 \pm 0.7$ \\
LSTM & $78.9 \pm 0.7$ & $93.1 \pm 0.6$ & $80.1 \pm 0.4$ & $88.4 \pm 0.1$ & $89.1 \pm 0.5$ \\
GRUs & $80.6 \pm 0.6$ & $93.3 \pm 0.7$ & $82.4 \pm 0.2$ & $89.1 \pm 0.5$ & $92.3 \pm 0.4$ \\
CNN & $81.4 \pm 0.3$ & $93.3 \pm 0.2$ & $83.5 \pm 0.3$ & $89.3 \pm 0.1$ & $92.5 \pm 0.5$ \\
AdaSent & $79.8 \pm 1.2$ & $92.2 \pm 1.2$ & $83.6 \pm 1.6$ & $90.4 \pm 0.7$ & $91.1 \pm 1.0$ \\
\hline TopCNN & $81.3 \pm 0.3$ & $93.5 \pm 0.2$ & $84.9 \pm 0.2$ & $89.9 \pm 0.2$ & $92.4 \pm 0.6$ \\
TopCNN $_{\text {sen }}$ & $81.1 \pm 0.4$ & $93.1 \pm 0.3$ & $84.5 \pm 0.3$ & $90.1 \pm 0.2$ & $92.1 \pm 0.2$ \\
TopCNN $_{\text {word\&sen }}$ & $82.4 \pm 0.1$ & $94.1 \pm 0.2$ & $85.3 \pm 0.2$ & $90.9 \pm 0.1$ & $93.5 \pm 0.3$ \\
TopCNN $_{\text {nes }}$ & $82.8 \pm 0.1$ & $94.7 \pm 0.1$ & $86.4 \pm 0.1$ & $91.5 \pm 0.1$ & $93.7 \pm 0.2$ \\
TopLSTMs $_{\text {word }}$ & $81.1 \pm 0.5$ & $93.8 \pm 0.6$ & $82.7 \pm 0.3$ & $89.9 \pm 0.2$ & $91.1 \pm 0.5$ \\
TopLSTMs $_{\text {sen }}$ & $80.4 \pm 1.1$ & $93.5 \pm 0.9$ & $81.7 \pm 0.5$ & $89.2 \pm 0.3$ & $90.3 \pm 0.8$ \\
TopLSTMs $_{\text {word\&sen }}$ & $80.6 \pm 0.7$ & $94.0 \pm 0.5$ & $82.2 \pm 0.3$ & $89.7 \pm 0.3$ & $91.5 \pm 0.4$ \\
TopLSTMs $_{\text {ens }}$ & $82.1 \pm 0.4$ & $94.4 \pm 0.5$ & $82.7 \pm 0.3$ & $91.1 \pm 0.2$ & $92.0 \pm 0.3$ \\
\hline
\end{tabular}

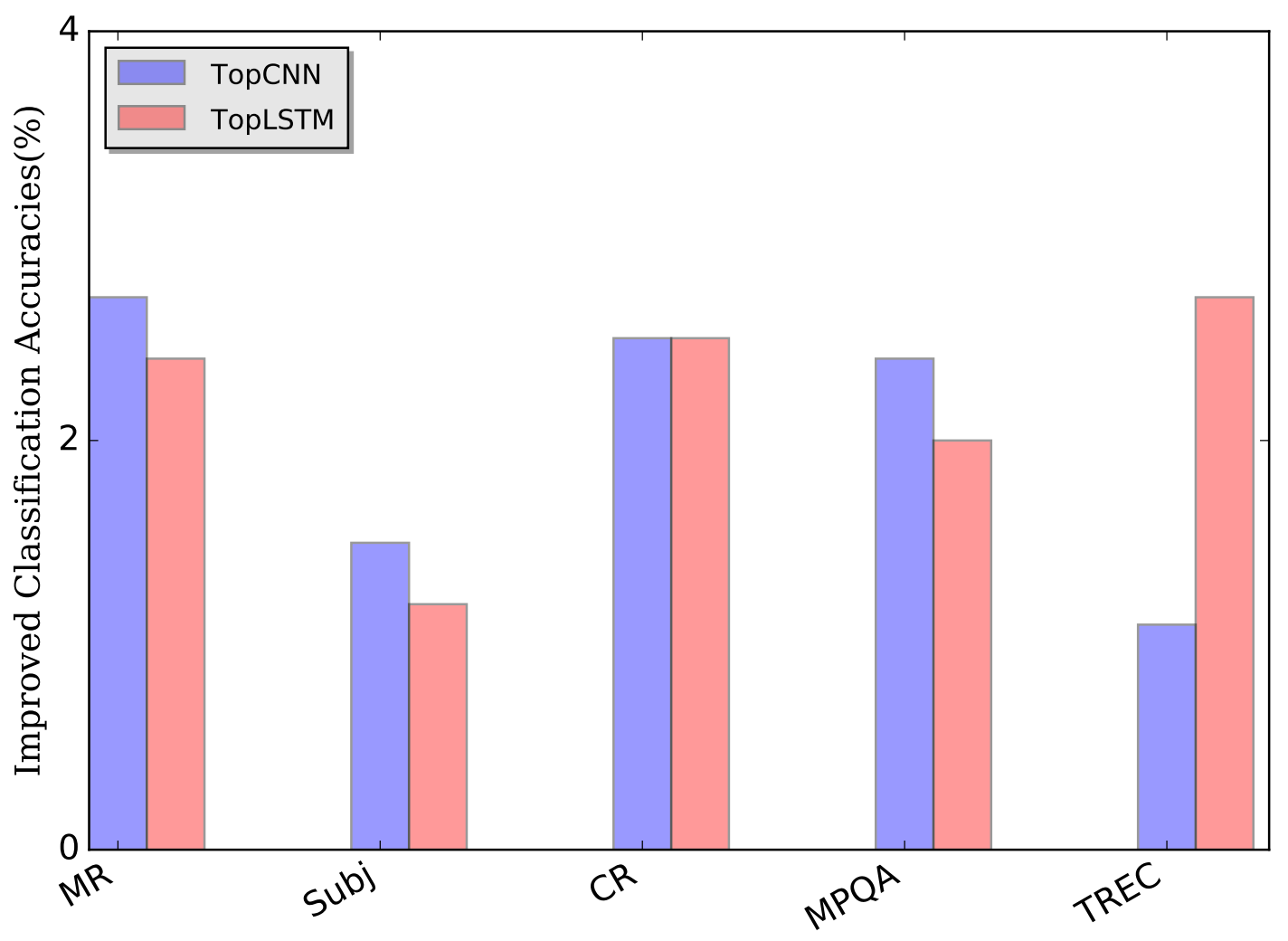

Figure 4.5: Performances gains achieved by our proposed TopCNN and TopLSTMs models compared to CNN and LSTM, respectively 


\subsubsection{Sensitivity to the Number of Topics}

The number of topics in $\mathrm{LDA}^{*}$ is a hyperparameter, which is vital to the performance of topic modeling. If the topic number is too small, the model would be too simple to capture the true topical distribution. On the other hand, a very large topic number may lead to the over-fitting problem due to the model's high complexity [119]. Since our proposed model integrates the topical probability into the semantic composition process, we next investigate the sensitivity of our models in response to the change of topic numbers based on the quality of learned representation on sentences.

Here, we only compared the performance of our proposed three TopCNN models including TopCNN $\mathrm{Nord}_{\text {wo }}$, TopCNN $\mathrm{Nen}_{\text {sen }}$ and TopCNN $\mathrm{Nord}_{\text {wsen }}$ under different numbers of topics over the five datasets. The topic number was set to 50, 100, 150, 200, 250, 300, respectively. We also compared the original CNN model without using topic models, which corresponds to zero topic number. Under different topic number, we run LDA* and our proposed joint neural model to learn topic embeddings. Therefore, for each dataset and each model, we had 7 different topic number settings and the other model parameters were fixed as the above reported setting and the other experimental settings are kept unchanged as reported above. From the results shown in Figures 4.6, 4.7, 4.8, 4.9 and 4.10 we have the following observations:

Firstly, TopCNN models with a moderate topic number achieve the best performance. This means that the integration of $\mathrm{LDA}^{*}$ into the semantic composition process is helpful for representation learning of sentence, and our proposed TopCNN models outperform the original CNN model under an appropriate topic number. It is noted that under large topic numbers, our proposed TopCNN models perform worse than the original CNN model. This may be because LDA* may not be able to infer topics behind short text under a large topic number. In addition, the increased dimensionality of word embeddings hinders the performance of CNN models as reflected in the performance comparison between CNN and $\mathrm{CNN}_{600}$. Here, an empirical study about number of topics has been conducted. In our future study, we plan to adopt some data-dependent measures to determine this hyper-parameter as shown in [120].

\subsubsection{Discussion on Topic Embeddings Learning}

The experimental results have shown that the joint neural model presented in Section 4.2.3 can lead to a good topic embeddings learning for our proposed models. In the joint neural model, the occurrence of the pair of word and topic is utilized via word2vec 


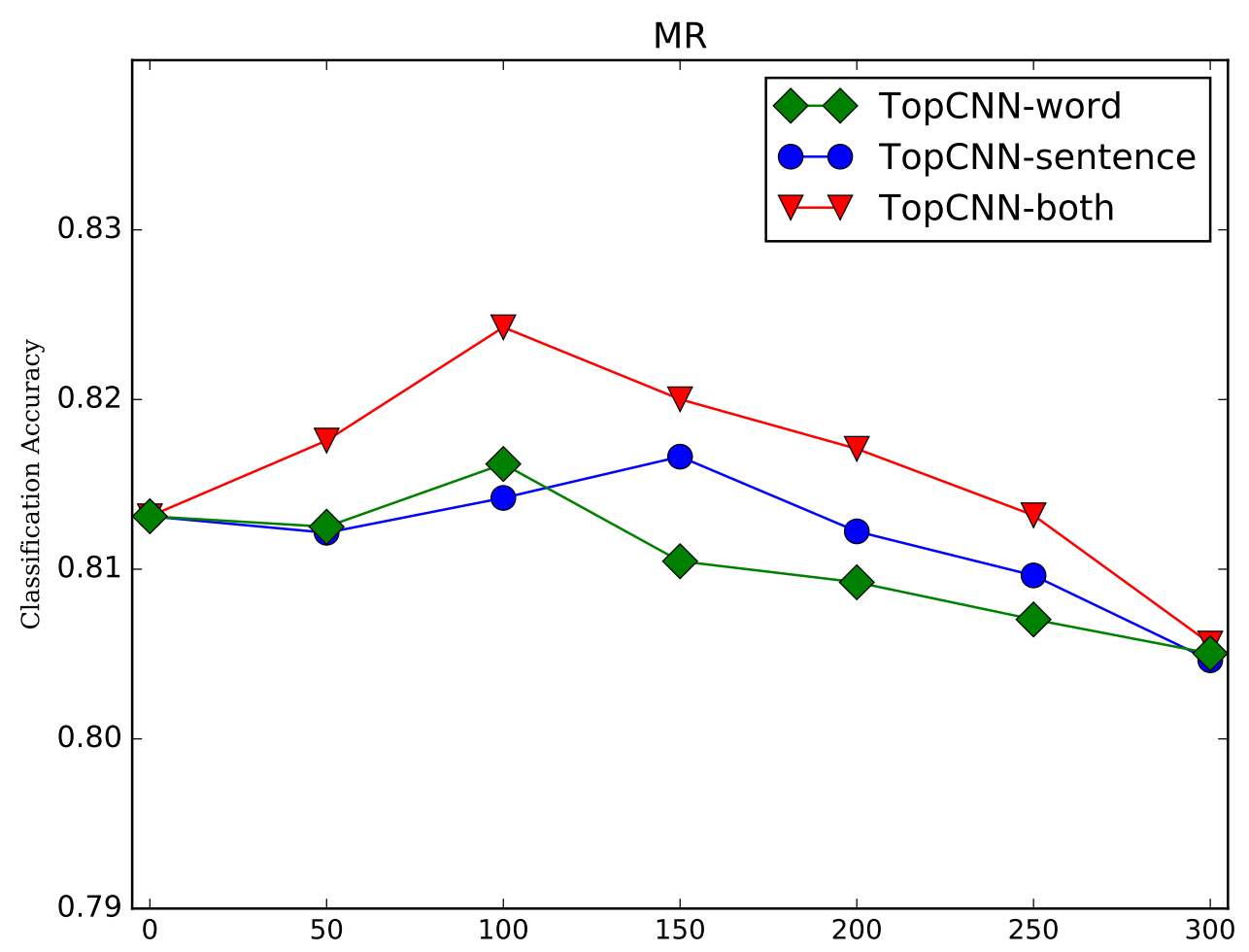

FiguRE 4.6: Performances of our proposed three models for different number of topics on MR dataset. The numbers of topics are ranged from 0 to 300 with a step size of 50 .

framework to learn topic embeddings. Therefore, semantic space of topics embeddings can be close to that of word embeddings. In Section 4.2.3, four topic embeddings learning schemes are proposed. Here, the four schemes incorporated in the proposed TopCNN are investigated experimentally.

For the random model, the topic embeddings were generated randomly following a uniform distribution in the range of $[-0.25,0.25]$. For the additive model, we average the embeddings of top 10 words for each topic as described in Eq. (4.13). For the topical neural model, based on the target function Eq .(4.15), the topic embeddings with a dimensionality of 300 were trained on the five datasets. Finally, the performance of our TopCNN model with four kinds of topics embeddings was compared. Here, we also randomly selected one folder for the first four datasets and for each settings, we run the same model ten times and recorded its corresponding mean value and standard deviation.

The results are shown in Figure 4.11. Firstly, the random topic embeddings perform the worst, while the performance difference is not significant. This may be due to the 


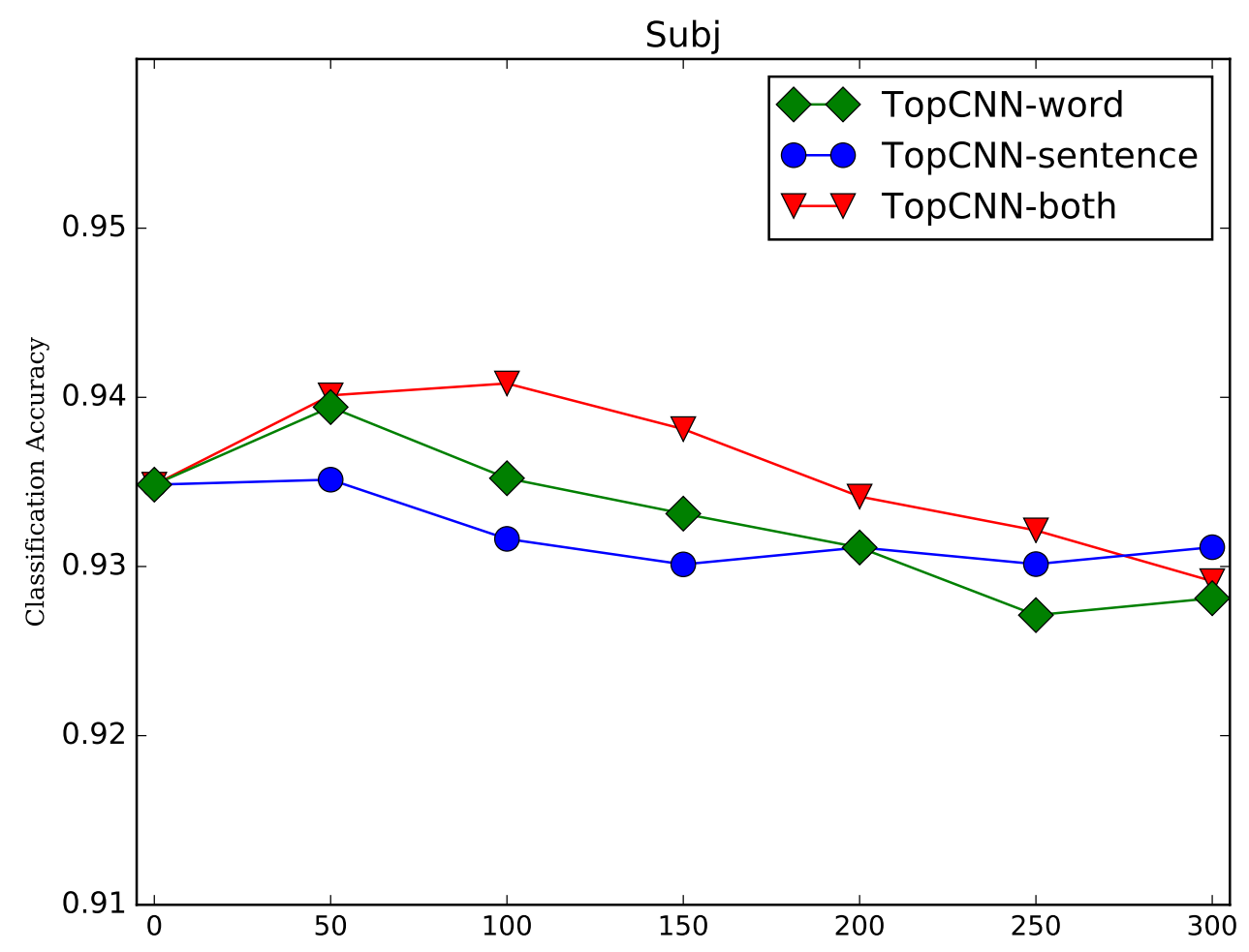

FiguRE 4.7: Performances of our proposed three models for different number of topics on Subj dataset. The numbers of topics are ranged from 0 to 300 with a step size of 50 .

adopted fine-tuning on topic embeddings. Figure 4.11 also shows that the topic embeddings learned from neural models do not consistently outperform over additive model on all datasets, despite that non-linear model has been proven to be successful in distributed representation learning. This may be due to the discrepancy of semantic spaces between topics and words. Word embeddings and topics embeddings are pre-trained independently. However, the additive model aims to derive the topic embeddings based on pre-trained word embeddings, and thus the semantic space discrepancy between words and topics may be eliminated. In addition, the adopted fine-tunning process may also be the reasons of comparable performance between additive embeddings and neural embeddings. Among the four kinds of topic embeddings, the contributions of joint neural model are the most significant with average performance gains of $1.78 \%$ and $1.25 \%$ compared to additive and topical models over these five datasets. This is because in the joint neural model, the topic and the associated word are observed together so that the embeddings for topics are learned with the word embeddings. As a result, the joint neural model yields the best performance. 


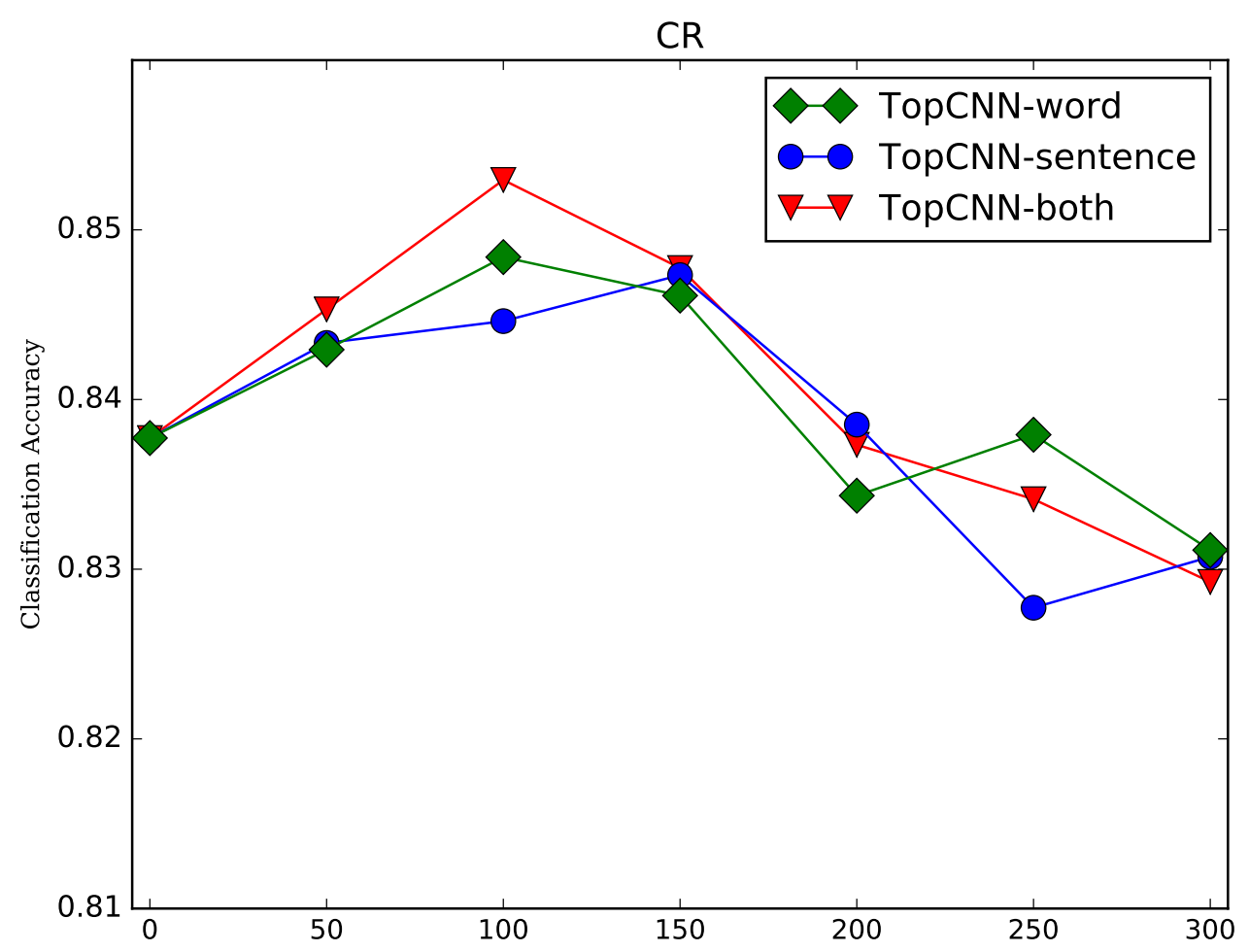

FiguRE 4.8: Performances of our proposed three models for different number of topics on CR dataset. The numbers of topics are ranged from 0 to 300 with a step size of 50 .

\subsection{Conclusion}

In this chapter, we have introduced a topic-aware deep compositional framework and developed two models named TopCNN and TopLSTMs for sentence representation. By incorporating probability distribution derived by LDA*, TopCNN and TopLSTMs are able to explore the topic-specific sense of words and sentences and further utilize such topic-specific sense jointly with general sense of word embeddings in the semantic compositional process. In addition, several effective architectures for topic embedding learning have been proposed and incorporated into our proposed framework. The experimental results have shown that TopCNN $\mathrm{N}_{\text {ens }}$ achieves competitive performances compared to the state-of-art models over five sentence classification tasks. The improved performances achieved by TopCNN and TopLSTMs over their original models have also proven the effectiveness of our framework.

Word embeddings can represent words in a low-dimensional and dense vector space, which makes the application of neural compositional model possible. In addition, word 


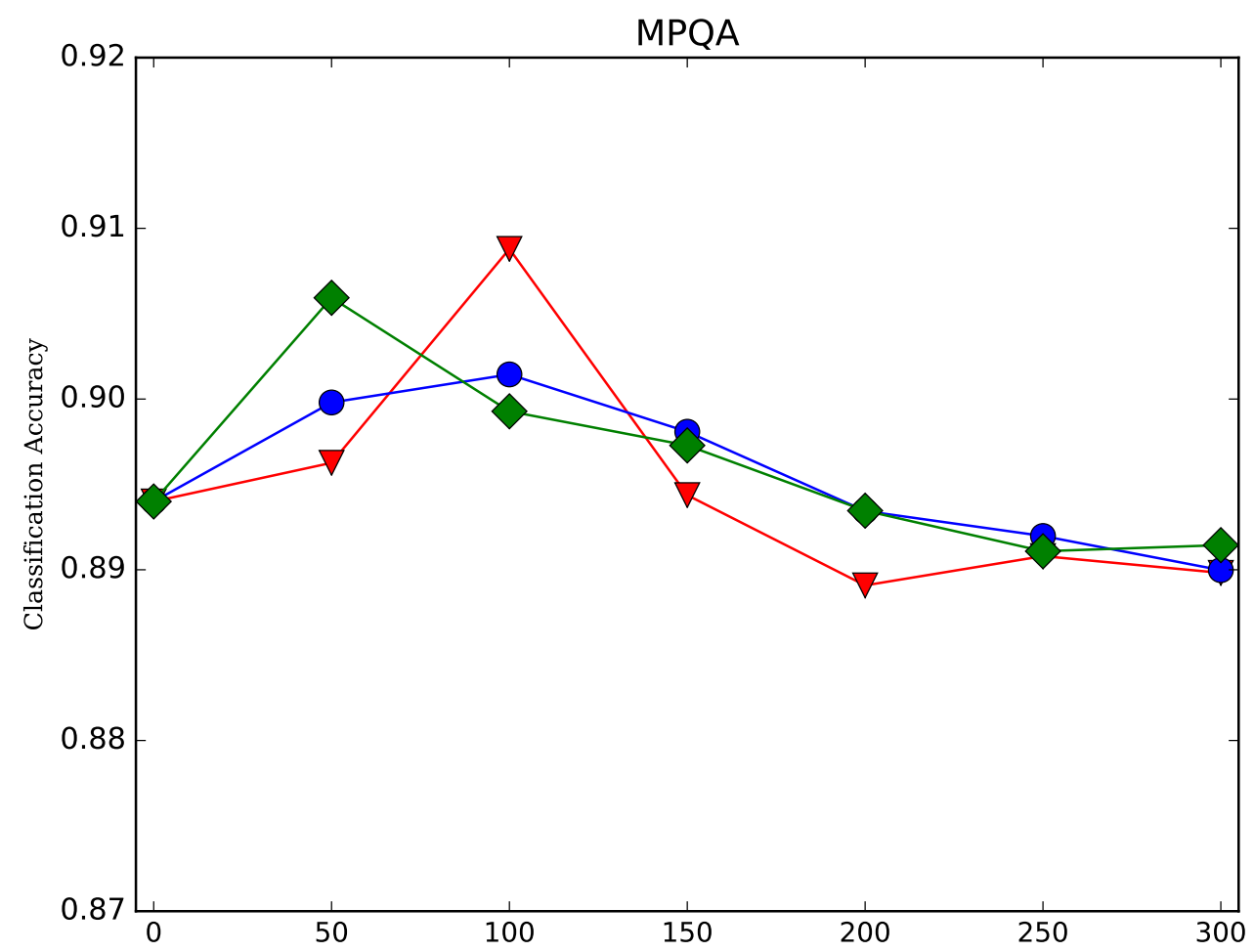

Figure 4.9: Performances of our proposed three models for different number of topics on MPQA dataset. The numbers of topics are ranged from 0 to 300 with a step size of 50 .

embeddings can also be used in the conventional text representation models. The next chapter will illustrate that the incorporation of word embeddings into BoW models can relieve the hard mapping assumption, i.e., exact word matching. 


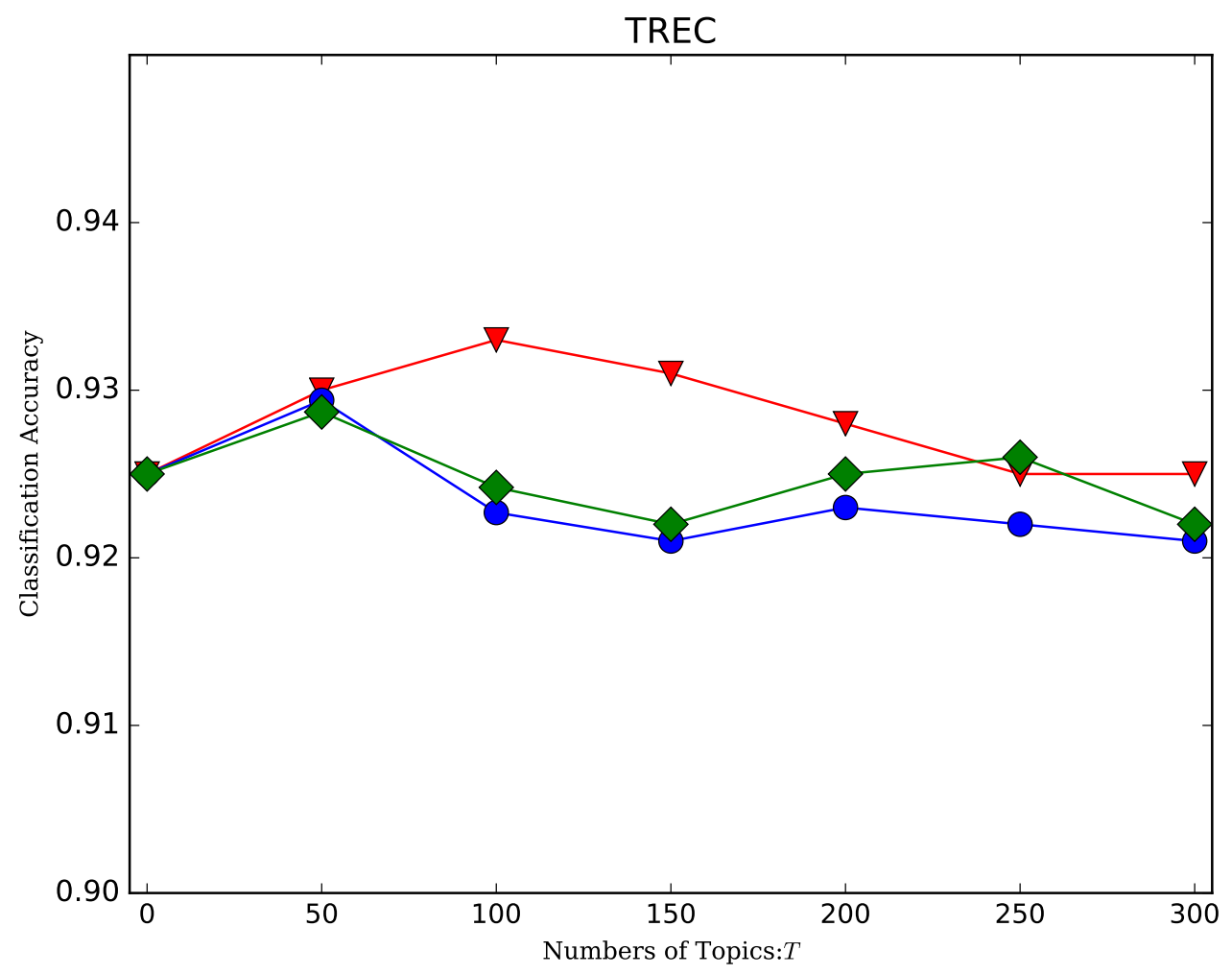

Figure 4.10: Performances of our proposed three models for different number of topics on TREC dataset. The numbers of topics are ranged from 0 to 300 with a step size of 50 . 


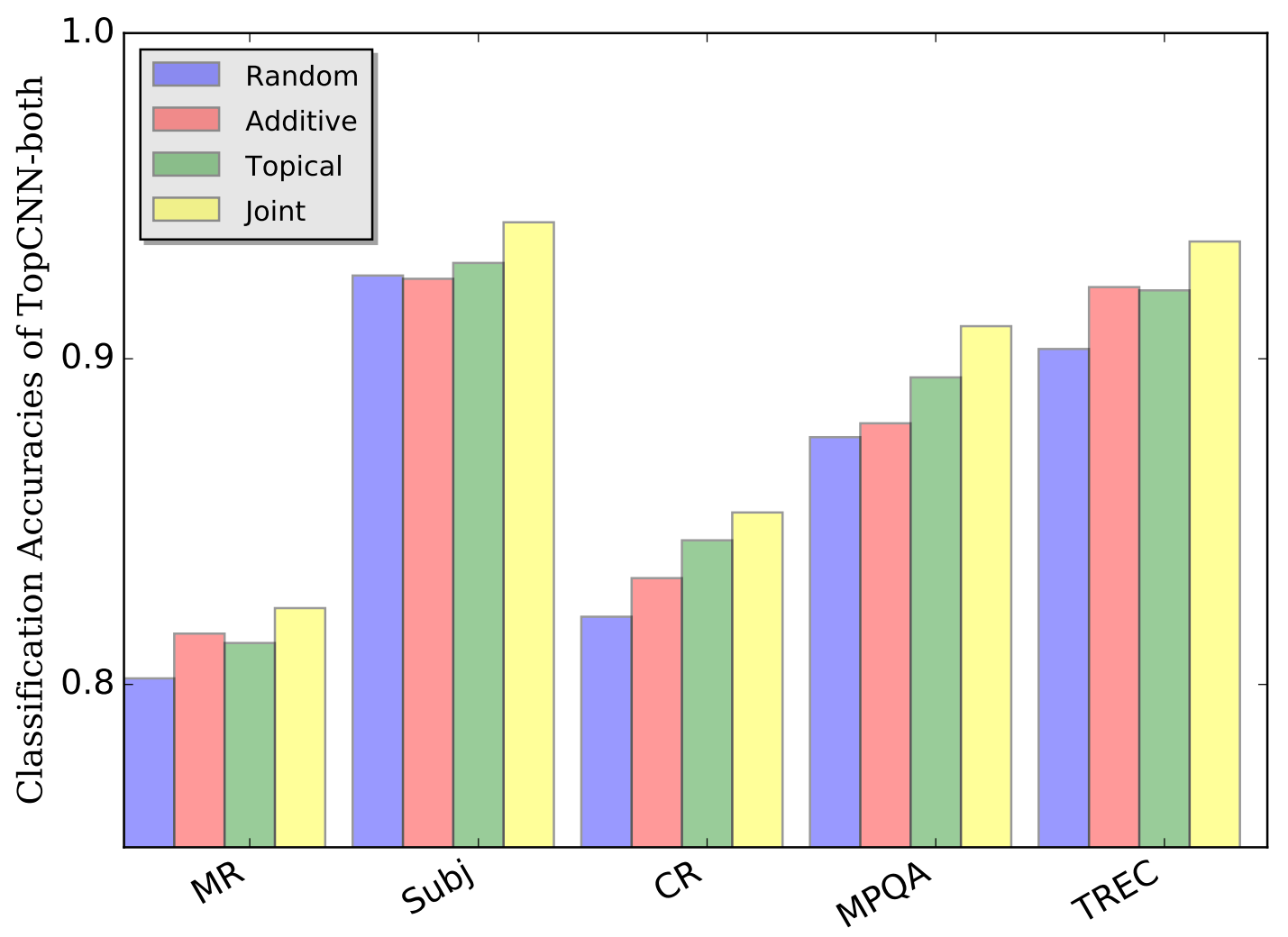

FiguRE 4.11: Effects of different topics embeddings learning schemes on the whole five datasets. 


\section{Chapter 5}

\section{Fuzzy Bag-of-Words Model for Document Representation}

The previous chapter introduced topic-aware deep compositional models for sentence modeling via enriching word embeddings. This chapter investigates the incorporation of word embeddings into the conventional BoW framework and discovers that our proposed models are not only connected to BoW-based models, but also very related to CNN-based neural compositional models. Here, we propose fuzzy BoW models to learn more dense and robust document representations encoding more semantics. Document classification tasks have been introduced to confirm our hypothesis.

This chapter is organized as follows. In Section 5.1, motivations behind our models and some previous work related to fuzzy systems are reviewed. Our proposed fuzzy BoW models for document representation are presented in Section 5.2. In Section 5.3, experimental results on document classification tasks are illustrated. Finally, concluding remarks are given in Section 5.4.

\subsection{Background}

In this section, motivations behind our proposed FBoW models are presented firstly. Then, the basic idea behind fuzzy systems and its applications to text mining are reviewed. 


\subsubsection{Motivations}

To overcome the limitations of the original BoW model as discussed above, we propose to replace the original hard mapping by a fuzzy mapping, and develop the Fuzzy BoW (FBoW) model. In contrast to BoW that employs exact word matching to basis terms, FBoW introduces vagueness in the matching between words and the basis terms. Fuzzy mapping enables a word semantically similar to a basis term to be activated in the BoW model. The membership function of a basis term in the FBoW model assigns membership values to words according to their semantic similarity to the basis term. The intuition behind such a membership function lies in that the membership values should be proportional to the semantic similarity between the word in documents and the basis terms. In our proposed model, word embeddings technique is introduced to help evaluate semantic similarity. Trained on a large corpus, word embeddings encode word meanings into vectors and thus the semantic similarity between two words can be conveniently evaluated using the cosine similarity between the corresponding word embeddings [53]. The cosine similarity measure can be interpreted as the degree of one word semantically matching another word. To illustrate the comparative advantages of our proposed fuzzy BoW over the original BoW, fuzzy BoW is applied to the same toy example, as shown in Figure 5.1.(b). Due to the adopted fuzzy mapping, bench in sentence $d_{1}$ and huskies in sentence $d_{2}$ can be mapped to the basis terms table and $\operatorname{dog}$ respectively, and their values are proportional to the semantic similarity. The fuzzy BoW produces the following vectors for the two toy sentences: $\boldsymbol{d}_{1}=(1,0.7,0.8,1)$ and $\boldsymbol{d}_{2}=(0.7,1,1,0.8)$. Obviously, the FBoW model produces two similar vectors for two semantically similar sentences. Based on FBoW, a fuzzy Bag-of-WordClusters (FBoWC) model is proposed. Different from fuzzy BoW (FBoW) model whose basis terms are single words, FBoWC uses clusters of words as the basis terms, where each cluster consists of semantically similar words. The fuzzy membership function is based on the similarity between words and word clusters. Three different similarity measures including mean, maximum and minimum between words and clusters are investigated, and this leads to three variants named $\mathrm{FBoWC}_{\text {mean }}, \mathrm{FBoWC}_{\max }$ and $\mathrm{FBoWC}_{\min }$, respectively. The main contributions of our work can be summarized as follows:

* Our proposed fuzzy Bag-of-Words (FBoW) model is able to reduce sparsity, improve robustness and encode more semantic information than the original BoW model. Instead of binary mapping in BoW, FBoW adopts fuzzy mapping, in which connections between words in documents and basis terms in BoW space are fuzzy numbers. Fuzzy membership value can be determined according to word semantic similarity, which can be easily calculated based on word embeddings in our model. 

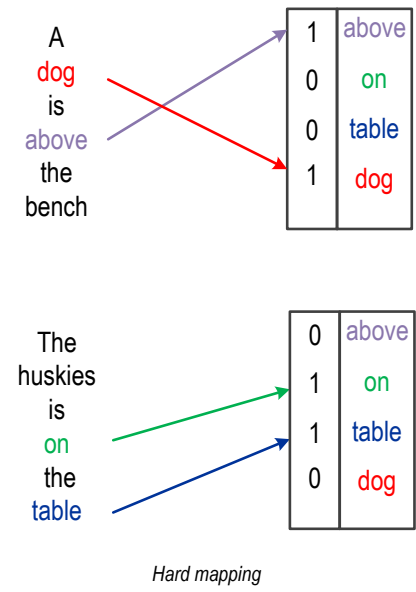

(a)

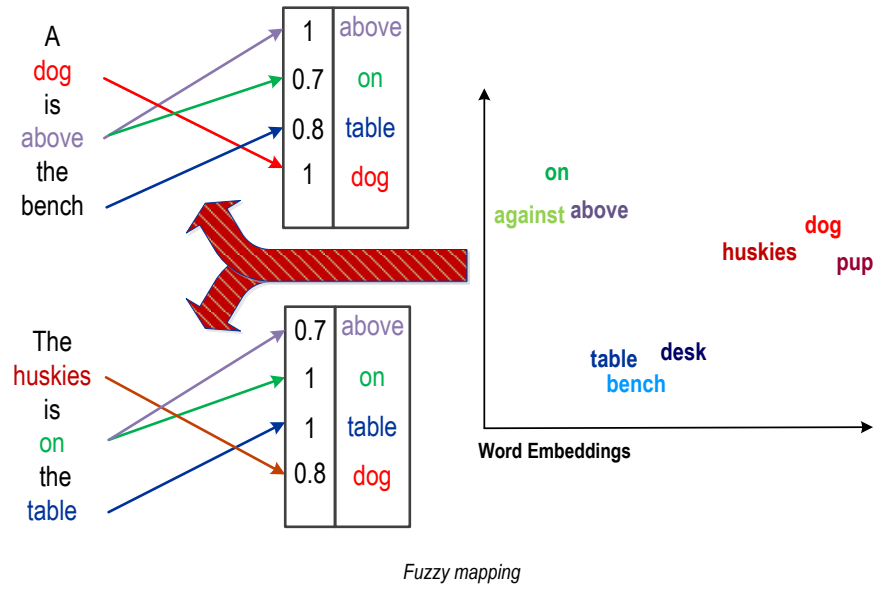

(b)

Figure 5.1: Illustration and Comparison between Bag-of-Words and our proposed Fuzzy Bag-of-Words models based on a toy example : (a) Bag-of-Words;

(b) Fuzzy Bag-of-Words.

By introducing fuzzy mapping, our proposed FBoW can be regarded as a more general formulation of BoW.

* The proposed fuzzy Bag-of-WordClusters model (FBoWC) produces representation with lower dimensions than FBoW. In FBoWC, words are clustered based on semantic similarity between words, and the word clusters are used as basis terms. Similar to FBoW model, a fuzzy membership function is constructed based on the similarity measures between words in corpus and clusters.

* Comprehensive experiments on several real-life document categorization datasets have verified the performance of our proposed models. Quantitative analysis is provided to prove that the representation learned by the FBoW and FBoWC models can capture more semantic information.

\subsubsection{Fuzzy System and Its Applications in Text Mining}

The core idea behind the fuzzy system is the employment of membership functions [121]. Fuzzy system is composed of three successive modules including fuzzification, inference, and defuzzification, which can be regarded as knowledge-based nonlinear system. Fuzzy system has been extensively applied in many fields, including automatic control, clustering, image processing, optimization and so on [122-126]. It should be noted that [123] also proposed a model named Fuzzy BoW model for computer vision tasks, which shares 
the same name with our work. The FBoW in [123] attempted to address feature vector ambiguity problem for image representation, while our FBoW solves semantic and expression diversity problem of natural language for document representation. In addition, our models are different from theirs in fuzzy membership constructions, motivations and design of codebook or basis terms under the context of documents. Since our work focuses on text data, some previous papers incorporating fuzzy logic into text mining problems are reviewed here [127-131]. Tjhi et.al [127] proposed a dual fuzzy-possibilistic coclustering for document clustering, in which document clusters and fuzzy-possibilistic word memberships functions are generated jointly. Chiang et.al [128] presented a hierarchical web document clustering method named fuzzy latent semantic clustering (FLSC). FLSC introduced co-occurring named entity associations to build a fuzzy linguistic topological space. Lee et.al [130] presented a fuzzy based method named ML-FRC to address multi-class text categorization problems. In ML-FRC, fuzzy transformation is adopted to obtain low-dimensional fuzzy relevance vectors and the fuzzy clusterings are linearly mapped to labels. Based on the linear mapping, a set of thresholds are computed on training corpus and used on testing corpus to classify unseen documents. Martin et.al [131] proposed fuzzy grammar fragments to extract structured grammar components from unstructured text. These above approaches all focused on classification and clustering methods for text data. However, our approaches address the representation learning in text mining. Jiang et.al [129] proposed a fuzzy self-constructing feature clustering algorithm (FFC) to learn text representation methods. The core idea that a cluster of words is regarded as a dimension for document vector behind FFC is partially similar to our proposed FBoWC. However, FFC is a supervised method that requires labels to generate word patterns and its applications are limited to multi-class document categorization. Our proposed FBoW models are able to derive document representation in an unsupervised way. Furthermore, the document vectors learned by our FBoW can be applied to various text mining applications including information retrieval, text categorization and text clustering etc.

\subsection{Fuzzy Bag-of-Words}

In this section, our proposed fuzzy Bag-of-Words models are presented. Since fuzzy membership function is constructed based on word embeddings, we begin with a brief review of word embeddings. 
TABLE 5.1: Top5 similar terms to words: book and student. They are retrieved based on word embeddings. Their corresponding cosine-similarity scores are also shown.

\begin{tabular}{c|c|c}
\hline Inquiry & Similar Words & Cosine Similarity Scores \\
\hline \multirow{5}{*}{ book } & tome & 0.748 \\
& books & 0.738 \\
& memoir & 0.730 \\
& paperback_edition & 0.686 \\
& autobiography & 0.674 \\
\hline \multirow{5}{*}{ student } & students & 0.729 \\
& teacher & 0.631 \\
& faculty & 0.608 \\
& school & 0.605 \\
& undergraduate & 0.602 \\
\hline
\end{tabular}

\subsubsection{Word Embeddings}

In the previous section 2.2, a detailed review of word embeddings has been given. The core idea behind word embeddings is to assign such a dense and low-dimensional vector representation to each word that semantically similar words are close to each other in the vector space. The merit of word embeddings is that the semantic similarity between two words can be conveniently evaluated based on the cosine similarity measure between corresponding vector representations of the two words. Here, we adopted the popular word embeddings word2vec $[17,19,53]$. Usually, the cosine similarity measure between word embeddings is used to measure the semantic similarity between two words:

$$
\cos \left(w_{i}, w_{j}\right)=\frac{\mathbf{w}_{\mathbf{i}} \cdot \mathbf{w}_{\mathbf{j}}}{\left\|\mathbf{w}_{\mathbf{i}}\right\|\left\|\mathbf{w}_{\mathbf{j}}\right\|}
$$

where $\mathbf{w}_{\mathbf{i}}$ and $\mathbf{w}_{\mathbf{j}}$ denote word embeddings of two words $w_{i}$ and $w_{j}$, respectively. The cosine similarity measure is positive when the words are close to each other, and is negative when the words have reverse meaning. The measure is zero under a pair of two completely random words. To give a illustration, the top 5 similar words to two example words book and student and their cosine similarity scores are given in Table 5.1. In our proposed FBoW models, cosine similarity measure based on word embeddings are utilized to construct fuzzy membership functions to map the words in documents to basis terms. 


\subsubsection{Fuzzy Bag-of-Words Model}

Firstly, some adopted notations in our proposed methods are introduced. Let $D=$ $\left\{w_{1}, \ldots, w_{v}\right\}$ be the vocabulary covering all the words existing in the text corpus, and $v$ is the vocabulary size. $\mathbf{W} \in \mathbb{R}^{v \times d}$ denotes a well-trained word embeddings matrix, where its $i$-th row $\mathbf{w}_{i} \in \mathbb{R}^{d}$ represents the $d$-dimensional word embeddings for word $w_{i}$. Each document in text corpus is represented by a BoW vector whose elements denote the number of occurrence of basis terms in the document. In a large corpus, only the top $l$ high-frequency words are usually selected as basis terms in BoW model to reduce the sparsity and dimensionality in BoW representations, and the BoW basis terms $T=\left\{t_{1}, \ldots, t_{l}\right\}$ is therefore a subset of the corpus vocabulary, i.e., $T \subset D$.

For traditional BoW representations, documents are mapped into vectors by exact matching of the words in the documents to the basis terms. Exact word matching is equivalent to performing a hard or crisp mapping. If a word $w$ matches a basis term $t_{i}$, the output of the crisp mapping function is 1 , and is zero otherwise. Exact word matching is equivalent to employing the following membership function:

$$
A_{t_{i}}(w)= \begin{cases}1 & \text { if } w \text { is } t_{i} \\ 0 & \text { otherwise }\end{cases}
$$

Figure 5.2. (a) also illustrates this hard mapping. BoW has two immediate impacts on document representations. First, the vector is very sparse. Second, the vector does not encode much semantic information. As illustrated in Figure 5.1, two semantic similar sentences with different word usages are mapped to two uncorrelated BoW vectors, which is counter intuitive.

Fuzzy Membership Function: To address the problem caused by exact word matching in BoW, we propose to use semantic matching, which matches two words based on semantic similarity. To implement semantic matching, we employ fuzzy membership function as shown in Figure 5.2.(b). The corresponding equation is given as follows:

$$
A_{t_{i}}(w)= \begin{cases}\cos \left(\mathbf{W}\left[t_{i}\right], \mathbf{W}[w]\right) & \text { if } \cos \left(\mathbf{W}\left[t_{i}\right], \mathbf{W}[w]\right)>0, \\ 0 & \text { otherwise, }\end{cases}
$$

where $\mathbf{W}[w]$ denotes word embeddings for word $w$. Here, we adopt word2vec as our word embeddings, and the fuzzy membership degree that measures the similarity between attribute (words in documents) and set (basis terms in BoW space) is approximated by their cosine similarity score. The membership function Eq. (5.3) is intuitive since only 
words that are semantically similar to basis terms (positive correlated) should be counted. In contrast to hard membership function that only counts words identical to basis terms, fuzzy membership function allows words semantically similar to the basis terms to be counted. As shown above, the fuzzy membership function of one basis term is determined by the cosine similarity between its corresponding word embeddings and other word embeddings. A word which is similar to basis terms should be mapped to a large degree of membership. In the following, we will present how the fuzzy membership function can be incorporated into the BoW model to provide a semantic document representation.

Representation Learning: Here, the fuzzy membership function is adopted to count the number of occurrence of basis terms in a document. For a document, the FBoW model representation is denoted by $\mathbf{z}=\left[z_{1}, z_{2}, \ldots, z_{l}\right]$, where the $i$-th element $z_{i}$ is the sum of the membership degrees that all words semantically match the $i$-th basis term, i.e.:

$$
z_{i}=c_{i} \sum_{w_{j} \in \mathbf{w}} A_{t_{i}}\left(w_{j}\right) x_{j}
$$

where $\mathbf{w}$ denotes a set of all words in the document, $t_{i}$ is the $i$-th basis term and $x_{j}$ denotes the number of occurrence of $w_{j}$. It should be noted that $c_{i}$ is a controlling parameter defined by different weighting schemes in BoW model. For example, $c_{i}=1$ if the counting scheme is adopted, while $c_{i}$ is the inverse-document frequency if the TF-IDF is adopted. For simplicity, we adopt the counting scheme as our weighting scheme and $c_{i}$ is fixed to 1. As shown in Eqs. (5.2) and (5.4), BoW model can be regarded as a special case of our proposed fuzzy model. In BoW, $x_{i}$ is determined by the term frequency only, which is equivalent to the utilization of hard membership function.

In the following, a matrix formulation of the above fuzzy BoW model is presented. The numerical vector representation of a document under fuzzy BoW model is given by:

$$
\mathbf{z}=\mathbf{x H}
$$

where

$$
\mathbf{x}=\left[x_{1}, x_{2}, \ldots, x_{v}\right]
$$

$x_{i}$ denotes the number of occurrence of word $w_{i}$ in the document;

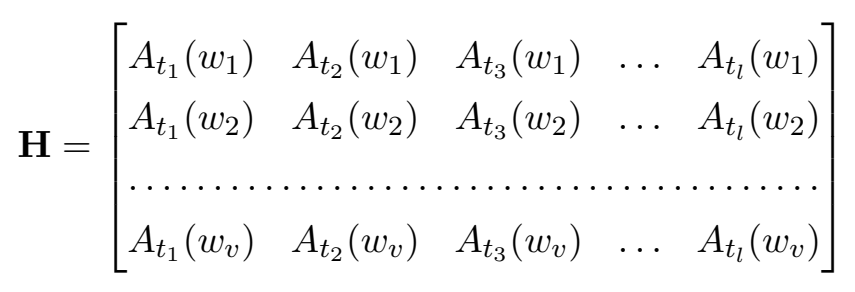




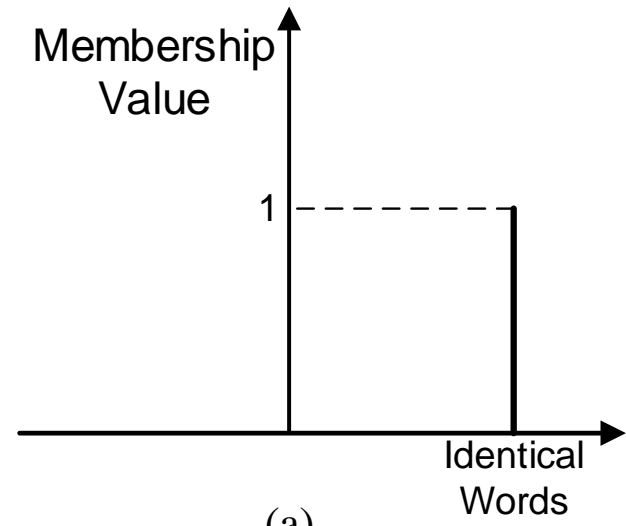

(a)

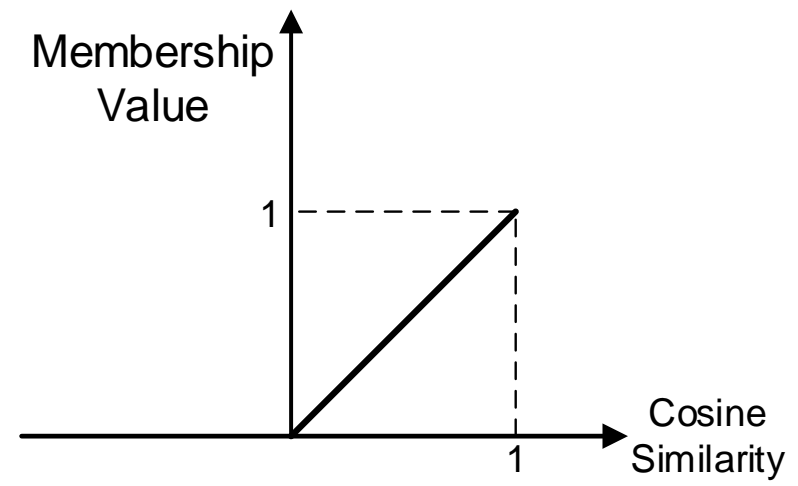

(b)

FiguRE 5.2: Illustrations of membership functions behind Bag-of-Words and our proposed Fuzzy Bag-of-Words models : (a) Bag-of-Words; (b) Fuzzy Bagof-Words.

where $A_{t_{i}}\left(w_{j}\right)$ is given by Eq. (5.3). Via Eq. (5.5), the final representation for document can be obtained. Each element of $\mathbf{x}$ is the weighted sum of word occurrence in the documents, where the weights are the membership values of the words in documents belonging to the corresponding basis terms in BoW model.

\subsubsection{Fuzzy Bag-of-WordClusters Model}

It is well acknowledged that BoW model has three limitations, including sparsity, high dimensionality, and lack of capability to encode high-level semantics. The fuzzy BoW model developed in Section 5.2.2 addressed the issues of sparsity and semantics, but the high dimensionality problem remains. Actually, the high dimensionality also means redundancy. This is the reason why BoW is often combined with LSA to reduce dimensionality. Certainly, FBoW can also be combined with LSA to reduce the dimensionality and redundancy of FBoW representation. In this study, we propose a plausible method to solve the high dimensionality and redundancy problem of FBoW model. The basic idea of the new method is to use word clusters instead of individual words as basis terms. A word cluster is a group of semantic similar words. Since basis terms are clustered, the dimension and redundancy can be significantly reduced. In FBoWC, even the entire vocabulary can be used as basis terms without increasing dimensionality of learned representations. We proposed three measures to evaluate the similarity between words and clusters and developed three variants of $\mathrm{FBoWC}$ models named as $\mathrm{FBoWC}_{\text {mean }}$, $\mathrm{FBoWC}_{\max }$ and $\mathrm{FBoWC}_{\min }$ by utilization of mean, max and min operation to obtain 
similarity between words and clusters, respectively. The three different similarity measures are illustrated in Figure 5.3, where max, min and mean scores are used to evaluate the similarity between a certain word and word cluster 1,2 and 3, respectively. The details of the three models are presented next.

Word Cluster Construction: Since word embeddings can capture the semantics of words, K-means clustering algorithm is applied to group these embeddings, i.e., discover word clusters. The number of clusters, which can be set by users, is equal to the dimensionality of the learned document representation.

Fuzzy Membership Function for $\mathrm{FBoWC}_{\text {mean }}$, $\mathrm{FBoWC}_{\max }$ and $\mathrm{FBoWC}_{\text {min }}$ : To derive fuzzy membership functions as given in Eq. (5.3), similarity measures between clusters and words should be defined. Here, three measures have been proposed, which lead to three models including $\mathrm{FBoWC}_{\text {mean }}, \mathrm{FBoWC}_{\max }$ and $\mathrm{FBoWC}_{\text {min }}$. It is assumed the $i$-th word cluster $\mathbf{t}_{i}$ contains a collection of word $\mathbf{t}_{i}=\left\{w_{i 1}, \ldots, w_{i k_{i}}\right\}$, where $k_{i}$ denotes the number of words in the $i$-th word cluster. To evaluate the similarity between cluster $\mathbf{t}_{i}$ and word $w$, we firstly compute the cosine-similarity between embeddings of words in cluster $\mathbf{t}_{i}$ and word $w$. Then, $\mathrm{FBoWC}_{\text {mean }}, \mathrm{FBoWC}_{\max }$ and $\mathrm{FBoWC}_{\min }$ take mean, maximum and minimum value, respectively:

$$
A_{\mathbf{t}_{i}}(w)= \begin{cases}\operatorname{mean}\left(\mathbf{q}_{\mathbf{t}_{i}}\right) & \text { for } \mathrm{FBoWC}_{\text {mean }}, \\ \max \left(\mathbf{q}_{\mathbf{t}_{i}}\right) & \text { for } \mathrm{FBoWC}_{\max } \\ \min \left(\mathbf{q}_{\mathbf{t}_{i}}\right) & \text { for } \mathrm{FBoWC}_{\min }\end{cases}
$$

where

$$
\begin{aligned}
\mathbf{q}_{\mathbf{t}_{i}}= & {\left[\max \left(\cos \left(\mathbf{W}\left[w_{i 1}\right], \mathbf{W}[w]\right), 0\right), \ldots\right.} \\
& \left.\max \left(\cos \left(\mathbf{W}\left[w_{i k_{i}}\right], \mathbf{W}[w]\right), 0\right)\right]
\end{aligned}
$$

$\mathbf{q}_{\mathbf{t}_{i}}$ is a vector whose elements are the similarity measures between words in cluster $\mathbf{t}_{i}$ and word $w$. In the max operation in $\mathrm{FBoWC}_{\max }$, the most similar word in word cluster $\mathbf{t}_{i}$ to word $w$ is identified, and the similarity measure with the most similar word is taken. This is to make sure that the similarity measure takes the value of 1 when the basis word is mapped to itself. Max operation has the effect of reducing intra-class spared in the dimension where the words in a word cluster are very relevant to the category of the document. Contrary to max operation, the min operation identifies the least similar word in a word cluster and takes the similarity measure with the least similar word in the word cluster as the output. Min operation also has the effect of reducing intra-class spread in the dimension where the words in the word cluster are NOT relevant to the category of the document. Both max and min operations have the effect of reducing intra-class spread, which is beneficial to pattern classification. The mean operation is 
equivalent to taking the similarity value with the centroid of a cluster, i.e. the word of a codebook in BoW for image representation [123].

The procedure of our proposed $\mathrm{FBoW}$ and $\mathrm{FBoWC}$ for document representation learning is given in Algorithm 2.

Algorithm 2 Fuzzy Bag-of-Words Frameworks

Input: a text corpus with $n$ documents; the vocabulary $\mathbf{D}$ and its corresponding word embeddings matrix $\mathbf{W} \in \mathbb{R}^{v \times d}$, where $v$ is the vocabulary size and $d$ is the dimensionality of word embeddings. Required dimensionality for document vectors: $l$

Output: learned document vectors for the corpus: $\mathbf{Z} \in \mathbb{R}^{n \times l}$

1: Based on the corpus vocabulary $\mathbf{D}$, obtain data matrix $\mathbf{X} \in \mathbb{R}^{n \times v}$ that each row $\mathbf{x} \in \mathbb{R}^{v}$ is the $i$-th document vector whose $j$-th element is the number of occurrence of word $w_{j}$ in the corresponding document, as shown in Eq. (5.6);

2: if $\mathrm{FBoW}$ is performed then

3: Based on term frequencies over the corpus, select the top- $l$ words with highest frequency as our models' BoW space $\mathbf{T}$ and the corresponding word embeddings are obtained as $\mathbf{W}_{T} \in \mathbb{R}^{L \times d}$;

4: $\quad$ Construct transformation matrix $\mathbf{H}$ based on $\mathbf{W}$ and $\mathbf{W}_{T}$ using Eqs. (5.3) and (5.7);

5: else if $\mathrm{FBoWC}$ is performed then

6: Apply K-means algorithm to cluster words based on word embeddings matrix $\mathbf{W}$ by setting the number of clusters to $l$. Then, the embeddings of words in each clusters are obtained and the cosine similarity between these clusters' words and word in documents are computed as shown in Eq. (5.9);

7: $\quad$ Construct transformation matrix $\mathbf{H}$ based on $\mathbf{W}$ and $\mathbf{q}_{\mathbf{t}_{i}}$ using Eqs. (5.8) and (5.7);

8: end if

9: Calculate learned data matrix $\mathbf{Z}$ according to Eq. (5.5), which can be used to represent the corpus.

10: return $\mathrm{Z}$

\subsubsection{Relationships with Previous Text Representation Meth- ods}

Word embeddings are introduced to capture the semantic relationships among words, and the derived semantic similarity and fuzzy mapping are then incorporated into the original BoW model. As a result, the learned document representations are more dense and able to capture more semantic information. In this subsection, we analyze the connections between our proposed FBoW frameworks including FBoW and FBoWC with two typical 


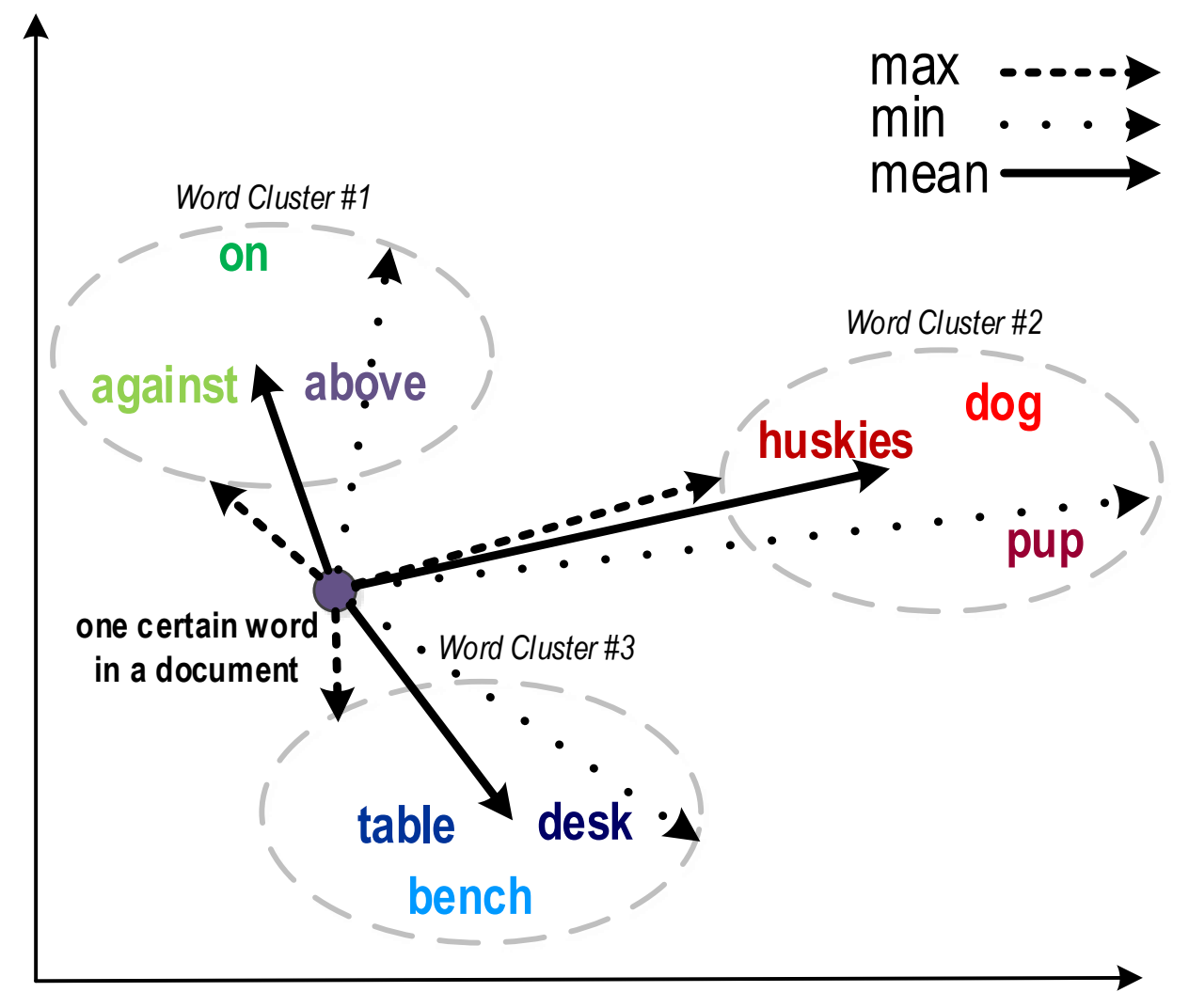

\section{Word Embeddings}

FigURE 5.3: Illustrations of mean, maximum and minimum similarity measures between clusters and words. It is noted that a high similarity measure denotes a small distance shown in the Figure.

text representation learning models including dimensionality reduction methods and a deep composition model: convolutional neural network (CNN).

Relationships with Dimensionality Reduction: Dimensionality reduction techniques seek to reduce the rank of vectors. Through dimensional reduction, sparse and high-dimension BoW vectors can be transformed into dense and low-dimensional ones, which in turn boosts the performance of subsequent tasks such as classification, information retrieval, etc. Some models including latent semantic analysis (LSA) and random projection (RP) are applied extensively in many text mining applications [32, 132]. LSA and RP are linear dimensionality reduction methods, and the key issue is to find the mapping matrix. For LSA, the mapping matrix is learned via maximizing the preservation of variance of the original feature space. Since the input information for LSA can be 
regarded as occurrence statistics between documents and words, LSA may fail to model the true semantic information and the resulting dimensions may not have interpretable meaning in natural language [133]. For RP, the mapping matrix is generated randomly. Some experimental results have shown that RP can achieve a significant speedup in computation time will little distortion of pairwise information of data. However, without data-based parameter tuning, RP may not capture the semantic information underlying the natural language.

In FBoWC representations, each dimension corresponds to word clusters which are subsets of the entire vocabulary. By contrast, each dimension in LSA and RP is a linear combination of all words in the vocabulary. As the mapping matrix of FBoWC in Eq. (5.7) directly measures the semantic similarity between words and basis terms based on word embeddings, it can capture high quality semantic information. In addition, word embeddings are pre-trained and publicly available, the computational cost is not a potential problem for FBoWC.

Relationships with Convolutional Neural Network: With the development of deep learning, recent works on text representation learning have been focused on deep compositional models [25, 28, 59]. The essence of deep compositional models is the application of multi-layers neural network of various forms including recursive [24], recurrent [26] and convolution [28] neural networks to learn and perform compositions over word embeddings. Among these models, convolutional neural network-based compositional models have been proven to be very efficient for sentence or short text modeling. Similar to CNN, the projection process adopted in our FBoW models can be regarded as a convolutional operation whose filter vector is the word embeddings of basis terms and window size is fixed to one gram. After sliding the filter through the whole sentence, the summation pooling method is adopted to get the activation of the filter. The output of each filter corresponding to each basis term are concatenated together to form the final document representation.

CNNs are suitable for sentence or short text modeling, but not suitable for document modeling due to the limits of window size. Our FBoW and FBoWC are effective for document or short text modeling. In addition, our approach belongs to unsupervised methods with limited computational cost, while CNN requires a large high-quality labeled training corpus. Furthermore, the filter parameters in CNN are tuned via back-propagation and the yielded representation lacks the semantic interpretability, but in our proposed FBoW and FBoWC, each dimension corresponds to a word or a cluster of words and is therefore interpretable. 


\section{$5.3 \quad$ Experiments}

In this section, we use document categorization tasks to evaluate the performance of our proposed Fuzzy Bag-of-words models.

\subsubsection{Descriptions of Datasets}

The task of document categorization is to assign a class label or category to a document. Seven real-life datasets are used in the experiments.

20Newsgroups is a collection of nearly 20,000 newsgroup documents, which is organized into 20 different classes. Here, we adopted the version of 20 Newsgroups (20NG) sorted by the removal of duplicates and some headers ${ }^{1}$. The whole corpus has 18846 documents, and the vocabulary size is 32716 , excluding the removed words whose document frequencies are less than five. Actually, the removal of low frequency words were performed for all the seven datasets used in the experiments. We followed predefined training and testing splitting. The statistics of $20 \mathrm{NG}$ are given in Table 5.2.

Reuters_14 and Reuters_8 were both generated from a classical corpus Reuters-21578 containing newswire articles and Reuters annotations ${ }^{2}$. The whole collection has 21,578 documents, which are categorized into 90 classes. Since some categories have only a few documents, we created two datasets containing 14 and 8 most frequent classes, respectively. The predefined training and testing splitting was adopted. The statistics of these two datasets Reuters_14 and Reuters_8 can be found in Table 5.2.

Amazon_6 is a collection of Amazon reviews for products of six categories. This dataset was originally published for sentiment analysis [134], but we used it for categorization. These six categories are cameras, laptops, mobile phone, tablets, TVs and video surveillance, in which the largest sample number is 6736 under cameras and the smallest sample number is 881 under tablets. To make the dataset more balanced, we randomly selected 1500 samples from categories with more than 1500 reviews. The corpus used in our experiments has 8083 reviews with a vocab size of 10790. The details are shown in Table 5.2 .

Amazon_4 is a multi-domain sentiment dataset, which contains product reviews crawled from Amazon for 4 product types: Kitchen, Books, DVDs and Electronics [135]. Here,

\footnotetext{
${ }^{1}$ The dataset has been kindly provided at http://qwone.com/ jason/20Newsgroups/

${ }^{2}$ The dataset has been kindly provided at http://csmining.org/index.php/ r52-and-r8-of-reuters-21578.html
} 
TABLE 5.2: Statistical Properties of Seven document categorization datasets.

\begin{tabular}{c|c|c|c|c|c}
\hline \multirow{2}{*}{ Datasets } & \multicolumn{5}{|c}{ Statistics } \\
\cline { 2 - 6 } & Vocab Size & Sample No. & Class No. & Average Length & Training/Testing \\
\hline 20NG & 32716 & 18846 & 20 & 349 & $11134 / 7532$ \\
\hline Reuters_14 & 9413 & 8286 & 14 & 105 & $5967 / 2319$ \\
\hline Reuters_8 & 8812 & 7674 & 8 & 102 & $5485 / 2189$ \\
\hline Amazon_6 & 10790 & 8083 & 6 & 395 & $4041 / 4042$ \\
\hline Amazon_4 & 9925 & 8000 & 4 & 40146 & $4000 / 4000$ \\
\hline BBC & 12473 & 2225 & 5 & 412 & $1112 / 1113$ \\
\hline BBCSport & 5345 & 737 & 5 & 370 & $368 / 369$ \\
\hline
\end{tabular}

we used it for classification of product types instead of sentiment analysis. The statistics of this dataset are given in Table 5.2.

$B B C$ is a collection of 2225 documents from the BBC news website [136]. The documents are crawled from 2004-2005 in five topical areas including business, entertainment, politics, sport and tech. The vocab size is 8865 .

BBCSport is a collection of 737 documents crawled from the BBC Sport website in five topical areas including athletics, cricket, football, rugby, tennis [136]. The vocab size is 3669.

Since training and testing subsets have not been given explicitly in their original sources in Amazon_6, Amazon_4, BBC and BBCSport, we split the corpus into training and testing domains equally. The train/test splitting was repeated 15 times. Therefore, for each corpus, 15 training/testing sub-datasets were created. The mean accuracy and standard deviation of each compared approach on 15 sub-datasets of each corpus were reported here.

To preprocess the above corpus, a English stop-word list built in the library sklearn ${ }^{3}$ was used for stop-word removal, and all characters were converted to lowercase because stop-word and case sensitivity may not be informative to document categorization tasks. In addition, stemming and lemmatization were not conducted, since words embeddings assign vectors to all forms of words instead of only their base forms.

\subsubsection{Experimental Setup}

Our proposed FBoW and FBoWC models are compared with the following document representation methods:

\footnotetext{
${ }^{3}$ http://scikit-learn.org/stable/modules/generated/sklearn.feature_extraction. text. CountVectorizer.html
} 
* BoW: the raw BoW representations are directly fed into the classifier. Here, the top high-frequency words, which is a subset of the vocabulary, are used as basis terms.

* BoW full: Different from BoW model, BoW full use the entire vocabulary as basis terms.

* LSA: Latent Semantic Analysis [44].

* LDA: Latent Dirichlet Allocation [102]. Our implementation of LDA is based on Gensim $^{4}$.

* AE: Average Embeddings take the average of word embeddings of all words contained in the document to represent the document.

* WMD: Word Mover's Distance [137]. The code is kindly provided 5 .

* FBoW and FBoWC (FBoWC ${ }_{\text {mean }}, \mathrm{FBoWC}_{\max }$ and $\left.\mathrm{FBoWC}_{\min }\right)$ : our proposed fuzzy bag-of-words models that utilize top high-frequency words and words clusters of the entire vocabulary as basis terms, respectively.

The input matrix for dimensionality reduction methods including LSA and LDA were both performed on $\mathrm{BoW}_{\text {full }}$ representation, which uses the entire vocabulary as the basis terms. For a fair comparison, FBoWC also used the clusters of entire vocabulary as basis terms. In BoW model, the top 2000 high-frequency words were used as basis terms and the resulting document representations have a dimensionality of 2000. For FBoW, the same top 2000 high-frequency words were used as basis terms. For LSA and LDA, the number of latent topics are both set to 2000. In LDA, we set hyperparameter $\alpha$ for document topic multinomial and hyperparameter $\eta$ for word topic multinomial to 1 and 0.01, respectively. For FBoWC, the number of clusters was set to 2000. Thus, the derived representations of LSA, LDA, FBoW and FBoWC have the same dimensionality of 2000 .

For AE, WMD, FBoW and FBoWC models, the same word embeddings were used. We utilized the pre-trained word2vec vectors published by Google ${ }^{6}$. These word embeddings were trained on a Google News corpus (over 100 billion words) and have a dimensionality of 300. For all the seven document categorization tasks, we further fine-tuned the pretrained word embeddings over the specific dataset. Since AE averages embeddings of all words, the dimension of document vector learned by $\mathrm{AE}$ is the same as the dimension of

\footnotetext{
${ }^{4}$ https://radimrehurek.com/gensim/index.html

${ }^{5}$ https://github.com/mkusner/wmd

${ }^{6}$ https://code.google.com/archive/p/word2vec/
} 
word embeddings, which is 300 . The other settings of WMD method were the same as that reported in its original paper [137].

Linear SVM [138] was applied to the document representations learned by the above mentioned approaches. In linear SVM, we searched the best regularization parameter C from $\{0.001,0.01,0.1,1$,

$10,100\}$. Since WMD can only derive document distance instead of document representations, document classification based on WMD used the $k \mathrm{NN}$ decision rule [139]. The searching range of the neighborhood size $k$ is $\{1,3, \ldots, 19\}$.

\subsubsection{Experimental Results on Document Categorization Tasks}

In this section, we compare the experiment results of our proposed FBoW and FBoWC models and several other benchmark document representation learning approaches on seven document classification datasets. The classification accuracies of each method on the seven datasets are shown in Table 5.3. For datasets Amazon_6, Amazon_4, BBC and BBCSport, the reported mean accuracies and standard deviations of all compared methods are over 15 random training/testing splittings.

First, it is observed that $\mathrm{BoW}_{\text {full }}$ model performs slightly worse than $\mathrm{BoW}$ model in five out of seven datasets. The difference between these two models lies in that $\mathrm{BoW}_{\text {full }}$ use all words in the vocabulary, while BoW only use top 2000 high-frequency words as basis terms. This means that the high dimensionality and the sparsity of $\mathrm{BoW}_{\text {full }}$ hinder its performance. Our approaches including FBoW and FBoWC all gain a significant performance improvement compared to original BoW models. This is because of the introduction of fuzzy mapping. Conventional BoW models assume all words are independent and adopt hard mapping, and this makes the learned document representations sparse and lack of high-level semantic information. By contrast, our proposed approaches adopt a fuzzy membership function and word embeddings to account for semantic correlation between words in a document and basis terms to learn dense and semantic document representations.

LSA, LDA and our proposed FBoWC models all are dimensionality reduction methods. For a fair comparison, the dimension of latent space in these methods is fixed to 2000. It can be seen that our proposed FBoWC models outperform LSA and LDA on all seven datasets. This is because our proposed FBoWC models utilize semantic matching and fuzzy membership function. 
The other two methods AE and WMD both employ word embeddings as our methods do. The results have shown AE and WMD are unable to achieve comparative performance. For AE, document representations are obtained via averaging the embeddings of all words occurred in the document. However, the averaging operation is too simple to model the complex semantic composition process from words to document. For WMD, the distance between words based on word embeddings is utilized to learn distance between documents instead of document representation, which limits its application. In document categorization applications, only distance-based classifiers such as $k \mathrm{NN}$ model can be used, while our proposed FBoW and FBoWC produce document representations that can be combined with any classifier such as SVM, which usually outperforms $k \mathrm{NN}$.

The performance of FBoW and FBoWC is also compared. FBoW adopts top 2000 high-frequency words as basis terms, while FBoWC use 2000 word clusters of the entire vocabulary as basis terms. As shown in Table 5.3, FBoWC models usually outperform FBoW. The better performance of FBoWC may be owing to two reasons. First, FBoWC representation is more informative than FBoW representation, though they have the same dimensionality, i.e., 2000 in the experiment. In FBoW, the 2000 dimensions correspond to the top 2000 high-frequency words, while the 2000 dimensions in FBoWC correspond to the entire vocabulary grouped into 2000 clusters. Second, FBoWC representations contain less redundancy. In FBoW, even two basis terms are semantically similar, they will create their own dimensions in the vector representation, while in FBoWC, semantically similar words are grouped to one cluster and derive only one dimension in the vector representation. Thus, FBoWC contains less redundancy than FBoW representation, which in turn produces better performance than FBoW. Among the three variants of FBoWC models, the performance of $\mathrm{FBoWC}_{\text {mean }}$ and $\mathrm{FBoWC}_{\max }$ over different datasets is more stable and robust than $\mathrm{FBoWC}_{\text {min }}$.

To further verify the significance of the better performance achieved by our methods, paired $t$-test was conducted to compare population means of two approaches, which is also illustrated in Chapter 3. Under these four adopted corpus including Amazon_4, Amazon_6, BBC and BBCSport, the best performing method proposed by us and the most competitive one from other 6 methods were selected for paired $t$-test. For each dataset, 15 random training/testing splittings were performed and the 15 training/testing splits were kept identical for all compared methods. The difference of mean accuracy and standard deviation of 15 results was computed as $\bar{d}$ and $\sigma_{d}$, respectively. Then, the $t$ statistic is calculated based on Eq. (3.11) with $n=15$. Under the null hypothesis that the performance difference of the methods pair is zero, the $t$-statistic follows a $t$-distribution with $n-1=14$ degrees of freedom. From the table of the $t$-distribution, p-values of the four selected method pairs are all less than 0.001, which means the best performing 
TABle 5.3: Classification Accuracies (\%) for Compared Methods on Seven Document Categorization Datasets. Bold Face Indicates Best Performance.

\begin{tabular}{|c|c|c|c|c|c|c|c|}
\hline Datasets & $20 N G$ & Reuters_8 & Reuters_14 & Amazon_4 & Amazon_6 & BBCSport & $B B C$ \\
\hline BoW & 68.0 & 96.6 & 96.0 & $91.3 \pm 0.3$ & $90.8 \pm 0.2$ & $98.1 \pm 0.7$ & $97.1 \pm 0.4$ \\
\hline $\mathrm{BoW}_{\text {full }}$ & 70.1 & 95.3 & 94.2 & $90.1 \pm 0.2$ & $90.9 \pm 0.2$ & $97.5 \pm 0.5$ & $96.5 \pm 0.4$ \\
\hline LDA & 73.2 & 96.2 & 92.8 & $91.1 \pm 0.3$ & $90.6 \pm 0.3$ & $97.1 \pm 0.6$ & $97.3 \pm 0.3$ \\
\hline LSA & 74.2 & 96.5 & 96.1 & $92.0 \pm 0.2$ & $91.0 \pm 0.3$ & $98.3 \pm 0.8$ & $97.3 \pm 0.4$ \\
\hline $\mathrm{AE}$ & 72.5 & 96.5 & 96.3 & $92.2 \pm 0.3$ & $91.1 \pm 0.3$ & $98.2 \pm 0.7$ & $97.2 \pm 0.3$ \\
\hline WMD & 74.1 & 94.5 & 96.1 & $92.1 \pm 0.3$ & $91.6 \pm 0.2$ & $97.6 \pm 0.5$ & $96.1 \pm 0.3$ \\
\hline FBoW & 77.7 & 97.1 & 96.7 & $93.5 \pm 0.2$ & $92.1 \pm 0.2$ & $98.5 \pm 0.8$ & $97.8 \pm 0.4$ \\
\hline $\mathrm{FBoWC}_{\text {mean }}$ & 78.9 & 97.2 & 97.1 & $93.5 \pm 0.3$ & $92.9 \pm 0.1$ & $98.5 \pm 0.7$ & $97.9 \pm 0.3$ \\
\hline $\mathrm{FBoWC}_{\max }$ & 78.5 & 97.3 & 97.0 & $93.9 \pm 0.3$ & $92.0 \pm 0.2$ & $98.9 \pm 0.8$ & $98.2 \pm 0.4$ \\
\hline $\mathrm{FBoWC}_{\min }$ & 75.5 & 97.5 & 97.2 & $93.5 \pm 0.3$ & $92.0 \pm 0.2$ & $98.3 \pm 0.8$ & $97.5 \pm 0.3$ \\
\hline
\end{tabular}

model in our proposed FBoW frameworks outperform the other comparative models at nearly $100 \%$ confidence level.

As mentioned above, the dimensionality of representations learned by various approaches is set to be 2000. To investigate the influences of this hyperparameter on our proposed FBoW models, we conducted $\mathrm{BoW}$ and $\mathrm{FBoWC}_{\text {mean }}$ on 20 newsgroups dataset under a range of dimensionality as $[500,2000,5000,8000,10000]$. Their accuracies are shown in Figure 5.4. The robustness of our proposed FBoW models has been verified considering our model outperforms BoW under different feature numbers. And our proposed $\mathrm{FBoWC}_{\text {mean }}$ achieves the best performance under a moderate dimensionality.

\subsubsection{Experimental Analysis of Mapping Bounds}

The membership function Eq. (5.3) is a special case of the following more general membership function with $\lambda=0$ :

$$
A_{t_{i}}(w)= \begin{cases}\cos \left(\mathbf{W}\left[t_{i}\right], \mathbf{W}[w]\right) & \text { if } \cos \left(\mathbf{W}\left[t_{i}\right], \mathbf{W}[w]\right)>\lambda \\ 0 & \text { otherwise }\end{cases}
$$

where $\lambda$ is the mapping bound or threshold. In the experiment, the influence of the parameter $\lambda$ on the performance of FBoW and FBoWC was investigated. Parameter $\lambda$ was set from 0 to 1 with a step size of 0.1 . $\mathrm{FBoWC}_{\min }$ is not considered here since the applied threshold $\lambda$ could easily set its fuzzy membership values to zero. Since clustering conducted in $\mathrm{FBoWC}_{\text {mean }}$ and $\mathrm{FBoWC}_{\max }$ requires random initialization, we performed the above two models five times and recorded their mean accuracies and standard deviations. Since the trends over all datasets are almost the same, we only show the results of Amazon_4 in Figure 5.5. It is observed when parameter $\lambda$ increases 


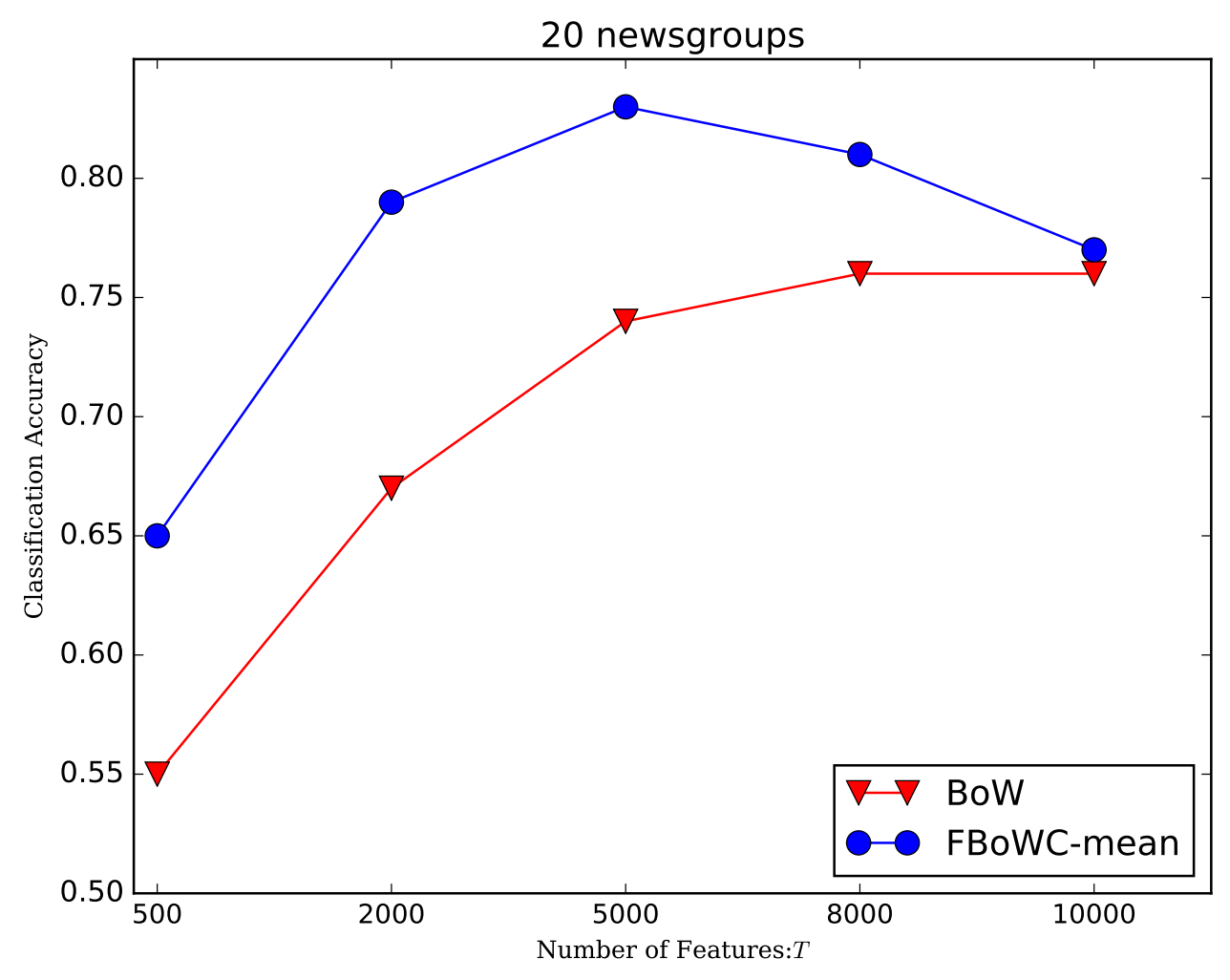

Figure 5.4: Performance of $\mathrm{BoW}$ and $\mathrm{FBoWC}_{\text {mean }}$ for different dimensionality $l$ on 20 newsgroups.

from 0 to 1 , the performance of our proposed approaches general degrades. FBoW, FBoWC $C_{\text {mean }}$ and $\mathrm{FBoWC}_{\max }$ all perform best when $\lambda=0$. The increased $\lambda$ increases the sparsity of the vector representation of a document. This means that the membership function Eq. (5.3) is effective. Another merit of Eq. (5.3) is that it is parameter-free and easy to use without requiring parameter tuning.

\subsection{Conclusion}

In this work, we have proposed Fuzzy Bag-of-Words models including FBoW and FBoWC to address sparsity and incapability of capturing high-level semantics behind documents. Word embeddings are utilized to measure semantic similarity among words and construct fuzzy membership functions of basis terms in BoW space over words in the task-specific corpus. Since word2vec embeddings can be trained over billions of words, word embeddings adopted in our methods are able to capture high-quality and meaningful semantic information that are not contained by the task-specific corpus alone. To determine basis 


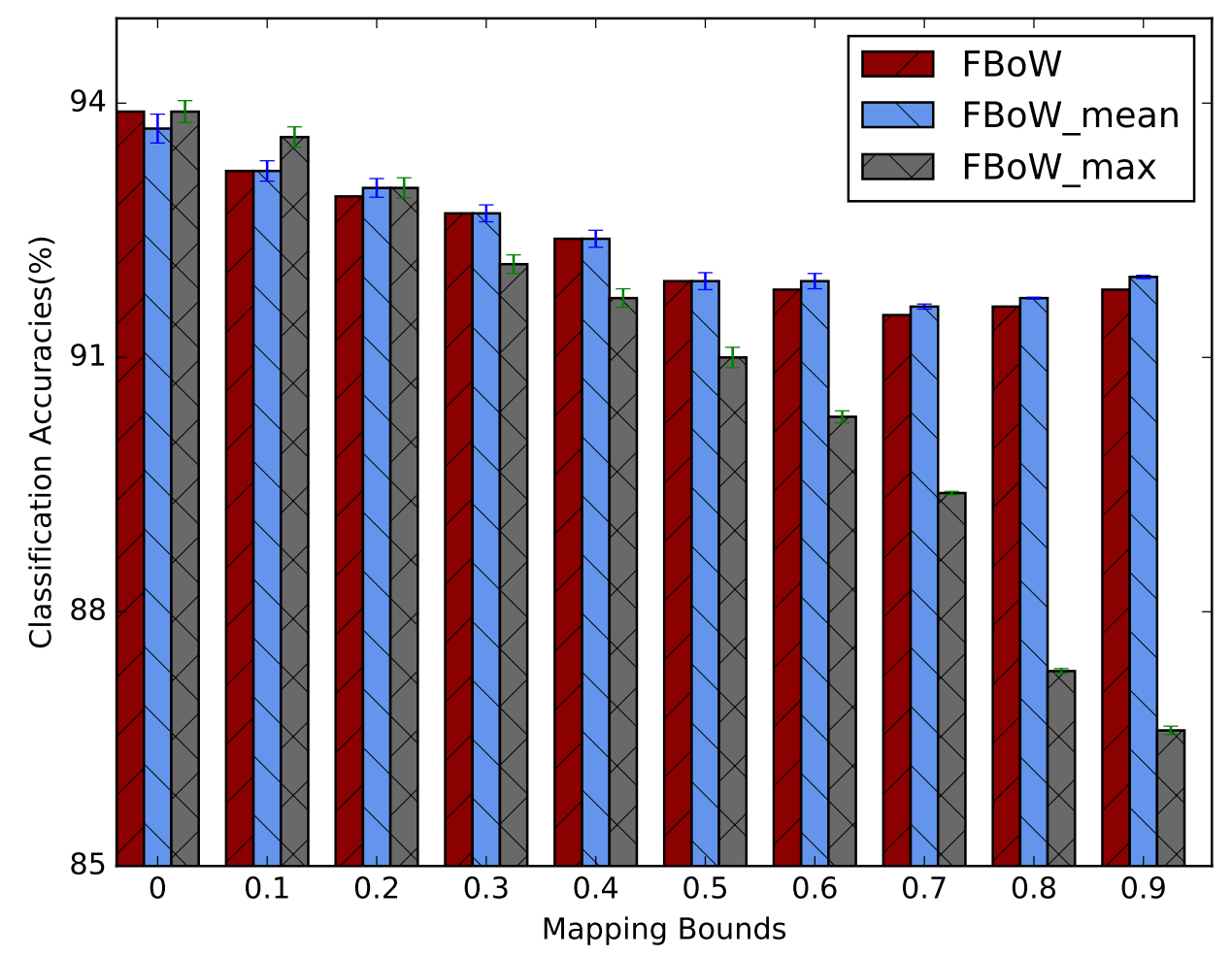

Figure 5.5: Performance of $\mathrm{FBoW}, \mathrm{FBoWC}_{\text {mean }}$ and $\mathrm{FBoWC}_{\max }$ for different mapping bounds: $\lambda$.

terms in BoW space, FBoWC utilizes word clusters, while FBoW directly regards high term-frequencies words as original BoW does. The adoption of word clusters in FBoWC can reduce feature redundancy and improve feature discrimination. Three different measures have been designed to evaluate similarity between clusters and words, and three corresponding variants of $\mathrm{FBoWC}$ models as $\mathrm{FBoWC}_{\text {mean }}, \mathrm{FBoWC}_{\max }$ and $\mathrm{FBoWC}_{\text {min }}$ have been developed.

The performance of our approaches has been experimentally verified through seven multiclass document categorization tasks. It has been shown that our proposed methods can be regarded as effective document representation learning methods. Since our proposed method is based on BoW framework, the order information of words can not be considered to model documents' semantics. As a next step work, document structure or word order information will be considered in document representation learning.

The next chapter will explore the application of robust text representation learning models for cyberbullying detection. Normally, in one specific domain, expert knowledge are available. Here, we focused on one domain: cyberbullying detection. The task is defined to detect whether a message is a bullying one or not. The expert knowledge is a list of 
bullying words. We will investigate how to utilize such expert knowledge to learn discriminative representations for message and detect cyberbullying effectively. Two methods will be proposed and extensive experiments will be conducted to verify the performances of our proposed methods. 



\section{Chapter 6}

\section{Representation Learning incorporating domain knowledge for Cyberbullying Detection}

Different from previous chapters that general text representation models are investigated, this chapter shows that it is meaningful to incorporate domain knowledge into text representation learning to address one specific problem: cyberbullying detection. Section 6.1 firstly explains the definition and importance of cyberbullying detection, and then reviews previous automatic cyberbullying detections methods based on text mining techniques. In sections 6.2 and 6.3, our proposed embedding-enhanced BoW model and semantic-enhanced marginalized stacked denoising auto-encoder are demonstrated, respectively. Section 6.4 provides a brief summary of these models for cyberbullying detection.

\subsection{Background}

\subsubsection{Motivations}

As defined in [140], social media is "a group of Internet-based applications that build on the ideological and technological foundations of Web 2.0, and that allow the creation and exchange of user-generated content." Via social media, people can enjoy enormous information, convenient communication experience and so on. However, social media 
may have some side effects such as cyberbullying, which may have negative impacts on the life of people, especially children and teenagers.

Cyberbullying can be defined as aggressive, intentional actions performed by an individual or a group of people via digital communication methods such as sending messages and posting comments against a victim. Different from traditional bullying that usually occurs at school during face-to-face communication, cyberbullying on social media can take place anywhere at any time. For bullies, they are free to hurt their peers' feelings because they do not need to face someone and can hide behind the Internet. For victims, they are easily exposed to harassment since all of us, especially youth, are constantly connected to Internet or social media. As reported in [141], cyberbullying victimization rate ranges from $10 \%$ to $40 \%$. In the United States, approximately $43 \%$ of teenagers were ever bullied on social media [142]. The same as traditional bullying, cyberbullying has negative, insidious and sweeping impacts on children [143-145]. The outcomes for victims under cyberbullying may even be tragic such as the occurrence of self-injurious behavior or suicides.

One way to address the cyberbullying problem is to automatically detect and promptly report bullying messages so that proper measures can be taken to prevent possible tragedies. Previous works on computational studies of bullying have shown that natural language processing and machine learning are powerful tools to study bullying [146, 147]. Cyberbullying detection can be formulated as a supervised learning problem. A classifier is first trained on a cyberbullying corpus labeled by humans, and the learned classifier is then used to recognize a bullying message. Three kinds of information including text, user demography, and social network features are often used in cyberbullying detection [148]. Since the text content is the most reliable, our work here focuses on text-based cyberbullying detection.

In the text-based cyberbullying detection, the first and also critical step is the numerical representation learning for text messages. In fact, representation learning of text is extensively studied in text mining, information retrieval and natural language processing (NLP). Bag-of-words (BoW) model is one commonly used model that each dimension corresponds to a term. Latent Semantic Analysis (LSA) and topic models are another popular text representation models, which are both based on BoW models. By mapping text units into fixed-length vectors, the learned representation can be further processed for numerous language processing tasks. Therefore, the useful representation should discover the meaning behind text units. In cyberbullying detection, the numerical representation for Internet messages should be robust and discriminative. Since messages on social media are often very short and contain a lot of informal language and misspellings, 
robust representations for these messages are required to reduce their ambiguity. Even worse, the lack of sufficient high-quality training data, i.e., data sparsity make the issue more challenging. Firstly, labeling data is labor intensive and time consuming. Secondly, cyberbullying is hard to describe and judge from a third view due to its intrinsic ambiguities. Thirdly, due to protection of Internet users and privacy issues, only a small portion of messages are left on the Internet, and most bullying posts are deleted. As a result, the trained classifier may not generalize well on testing messages that contain nonactivated but discriminative features. The goal of this study presented in this chapter is to develop methods that can learn robust and discriminative representations to tackle the above problems in cyberbullying detection.

In cyberbullying detection, some bullying messages always contain insulting or curse words, which can be regarded as discriminative features. This semantic information can be utilized as domain knowledge. Our proposed approaches provide elaborated usages of bullying terms. In this chapter, we introduce two approaches including Embeddingsenhanced Bag-of-Words Model (EBoW) and Semantic-enhanced marginalized stacked denoising auto-encoder (smSDA).

\subsubsection{Related Works}

With the increasing popularity of social media in recent years, cyberbullying has emerged as a serious problem afflicting children and young adults. Previous studies of cyberbullying focused on extensive surveys and its psychological effects on victims, and were mainly conducted by social scientists and psychologists [145, 149-151]. Although these efforts facilitate our understanding for cyberbullying, the psychological science approach based on personal surveys is very time-consuming and may not be suitable for automatic detection of cyberbullying. Since machine learning is gaining increased popularity in recent years, the computational study of cyberbullying has attracted the interest of researchers. Several research areas including topic detection and affective analysis are closely related to cyberbullying detection. Owing to their efforts, automatic cyberbullying detection is becoming possible. In machine learning-based cyberbullying detection, there are two issues: 1) text representation learning to transform each post/message into a numerical vector and 2) classifier training. Xu et.al presented several off-the-shelf NLP solutions including BoW models, LSA and LDA for representation learning to capture bullying signals in social media [147]. As an introductory work, they did not develop specialized models for cyberbullying detection. Yin et.al proposed to combine BoW features, sentiment feature and contextual features to train a classifier for detecting possible harassing posts [152]. The introduction of the sentiment and contextual features has been 
proven to be effective. Dinakar et.al used linear discriminative analysis to learn label specific features and combine them with BoW features to train a classifier [153]. The performance of label-specific features largely depends on the size of training corpus. In addition, they need to construct a bullyspace knowledge base to boost the performance of natural language processing methods. Although the incorporation of knowledge base can achieve a performance improvement, the construction of a complete and general one is labor-consuming. Nahar et.al proposed to scale bullying words by a factor of two in the original $\mathrm{BoW}$ features [154]. The motivation behind this work is quit similar to that of our model to enhance bullying features. However, the scaling operation in [154] is quite arbitrary. Ptaszynski et.al searched sophisticated patterns in a brute-force way [155]. The weights for each extracted pattern need to be calculated based on annotated training corpus, and thus the performance may not be guaranteed if the training corpus has a limited size. Besides content-based information, Maral et.al also employ users' information, such as gender and history messages, and context information as extra features [156, 157]. Huang et.al also considered social network features to learn the features for cyberbullying detection [148]. The shared deficiency among these forementioned approaches is constructed text features are still from BoW representation, which has been criticized for its inherent over-sparsity and failure to capture semantic structure $[44,102,119]$.

\subsection{Embeddings-enhanced Bag-of-Words Model}

In this section, we proposed a new representation learning method for cyberbullying detection named embeddings enhanced Bag-of-Words model (EBoW). In EBoW, we firstly define a list of insulting words based on expert knowledge and linguistic resources, i.e., insulting seeds. Then, based on word embeddings, we extend insulting seeds to define bullying features. Different weights are assigned to bullying features based on the cosine similarity between word embeddings. Considering insulting seeds are expanded through word embeddings, the construction of insulting seeds do not require much manual labor. Finally, we concatenate the learned bullying features with BoW features and latent semantic features to form the vector representation.

\subsubsection{EBoW: Embeddings-enhanced Bag-of-Words Model}

The final representation dervied by EBoW consists of three parts including Bag-of-Words features, latent semantic features and bullying features based on word embeddings, as 
shown in Figure 6.1. These three kinds of features are presented as follows, in particular, the details of bullying features generation.

BoW Features To extract Bag-of-Words features, a vocabulary including unigram and bigram is constructed firstly and the terms whose document frequencies are less than 2 are all ignored. Different term weighting schemes including $t f-i d f$ and binary ones can be applied here $[13,158]$. Here, we adopt the $t f-i d f$ weighting scheme. The $t f-i d f$ weight corresponding to the $i$-th word in $j$-th document is calculated as follows:

$$
w_{i, j}=T F_{i, j} \times \log \left(\frac{N}{D F_{i}}\right)
$$

where $T F_{i, j}$ is the term frequency of the $i$-th word in $j$-th document, $D F_{i}$ is the number of documents containing $i$-th word and $N$ is the number of documents.

Latent Semantic Features Here, latent semantic features refer to the features extracted by Latent Semantic Analysis (LSA) [44]. In principle, LSA applies Singular Value Decomposition (SVD) on the term-document matrix that each column is the above Bag-of-Words features. Then, the derived latent space is spanned by dominant eigenvectors corresponding to large eigenvalues. Each new feature is a linear combination of all original features.

Bullying Features Insulting words can be pre-defined and extracted based on our prior knowledge and other public linguistic resources, which are named insulting seeds. Then, we extend these insulting words automatically based on word embeddings. The insulting words and their corresponding extended terms serve as bullying features.

Since some cyberbullying messages usually contain curse or insulting words, these words are good indications of the existence of bullying. Therefore, we select a list of insulting words based on our prior knowledge and some external linguistic resources ${ }^{1}$. This list contains 350 words indicating curse or negative emotions, such as nigga, bitch, fuck, slut, whore, twat etc. Then, we compare the word list with the unigram and bigram features of the corpus used in experiments to obtain the intersection, which is regarded as insulting seeds. As discussed in the previous work [154], these features are discriminative for cyberbullying detection.

These insulting words are used to construct a vocabulary, and each Internet message is mapped to a vector by counting the occurrence times of each word appears. Different term

\footnotetext{
${ }^{1} \mathrm{~A}$ collection of insulting words can be found in the website: http://www. noswearing.com/ dictionary
} 
weighting schemes including $t f-i d f$ and binary ones are applied. However, such a direct use of these insulting words could hardly achieve robust representation. The reasons are two-fold. Firstly, due to the linguistic complexity and the synonymy, the obtained insulting words can not cover all the possible bullying terms. Secondly, effect intensities of different insulting words are different. The occurrence of slut is more indicative of the existence of cyberbullying than that of nerd, although these two words both express negative affection. To overcome these two limitations, we employ word embeddings to extend these insulting words and weight them.

Different from the one-hot representation in Bag-of-Words model, word embeddings use real-valued and low-dimensional vectors to represent semantics of words. In the vector space behind well-trained word embeddings, similar words are placed close to each other. For example, the words China and Singapore are close to each other, and the words beef and pork are close to each other. The cosine similarity between word embeddings can represent the semantic similarity between the two corresponding words. Since we focus on twitter corpus here, we utilize a well-trained word2vec model on a large-scale twitter corpus including 400 million tweets [159]. A visualization of some word embeddings based on PCA is shown in Figure 6.2. It is observed that curse words form distinct clusters, which are also far away from normal words. Even insulting words are located at different regions due to different word usages and insulting expression.

We extend the pre-defined insulting seeds based on word embeddings. For each insulting seed, we select the top- $h$ most-similar words in the vocabulary as extended bullying features. Based on word embeddings, the cosine similarity of the two embeddings can measure the similarity between these two words. It should be noted that the vocabulary of our corpus consists of unigrams and bigrams. For one bigram $w_{l} w_{r}$, we simply use an additive model to derive the corresponding embedding as follows:

$$
\mathbf{v}\left(w_{l} w_{r}\right)=\mathbf{v}\left(w_{l}\right)+\mathbf{v}\left(w_{r}\right)
$$

To give a clear illustration, the top-10 similar terms to the insulting seed: "slut" and their cosine similarities scores are shown in Table 6.1. These cosine similarity scores can be used to weight these extended features. And the weights of these insulting seeds are set to 1 . These extended features and insulting seeds compose the final bullying features. It should be noted that one word may be similar to several insulting seeds. To address the possible overlapping, we only keep the highest cosine similarity of the word as its weight. Therefore, the length of the final bullying features is less than or equal to $n * h$ where $n$ is the number of insulting seeds. 
TABLE 6.1: Top-10 similar terms to the insulting word: slut. They are retrieved based on word embeddings. Their corresponding cosine-similarity scores are also shown.

\begin{tabular}{c|c}
\hline Similar Words & Cosine Similarity Scores \\
\hline a slut & 0.815 \\
\hline whore & 0.738 \\
\hline a whore & 0.638 \\
\hline hypocrite & 0.536 \\
\hline bitch & 0.508 \\
\hline puta & 0.468 \\
\hline nerd & 0.455 \\
\hline bully her & 0.451 \\
\hline fat bully & 0.440 \\
\hline bully nigga & 0.435
\end{tabular}

Final Representation

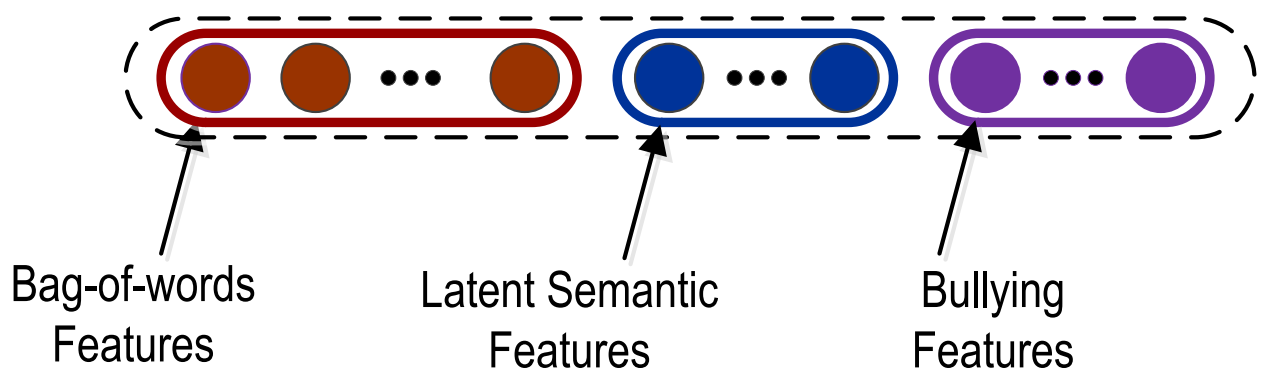

FiguRE 6.1: Illustration of final representation in our proposed EBoW model.

Feature Concatenation The three above kinds of features including Bag-of-Words features, latent semantic features and bullying features are concatenated to form the final representation. When final representation for each Internet message is obtained, a classifier is built to detect the existence of cyberbullying. In our proposed system, we feed learned features into a linear SVM pattern classifier.

\subsubsection{Experiments}

In the following, we evaluate our proposed EBoW model using a real-world cyberbullying dataset. The details of our adopted dataset and experimental settings are introduced firstly. Experimental results and comparison with other baseline methods are followed. 


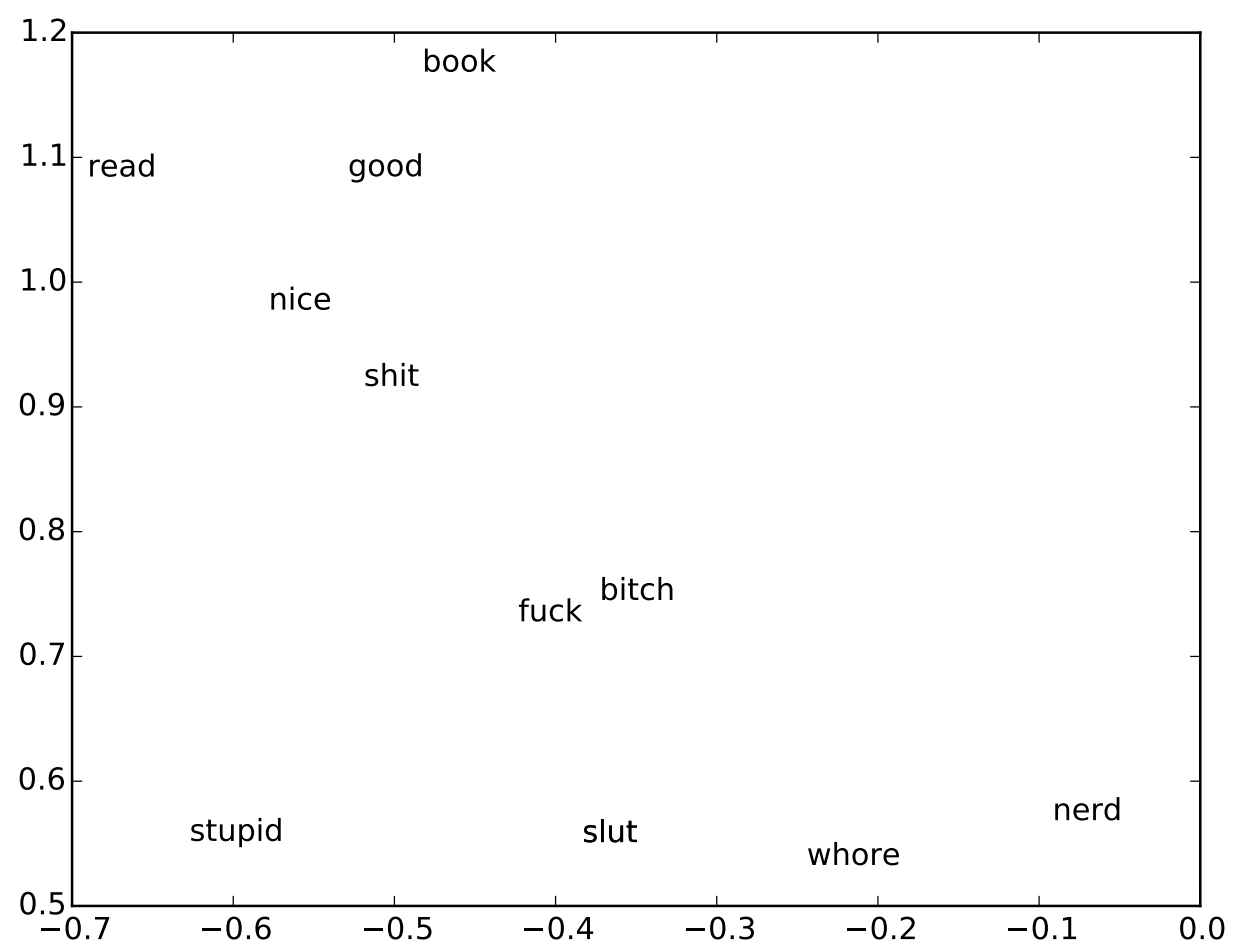

Figure 6.2: Two dimensional visualization of our used word embeddings via PCA. Displayed terms include both bullying ones and normal ones. It shows that similar words are nearby vectors.

Descriptions of Datasets The public bullying traces dataset is adopted here ${ }^{2}$, which consists of tweets that are messages sent on Twitter [147]. Twitter is a very popular social media application, which is able to let you access to the latest stories, ideas, opinions and news(https://about.twitter.com/). Registered users can read and post tweets, which are defined as the messages posted on Twitter with a maximum length of 140character. Twitter has over 270 million active users, and 500 million tweets are posted per day. Behind these amazing statistics, cyberbullying has sadly become a common occurrence. According to a previous study ${ }^{3}$, there were about 100,000 abusive tweets a week. Therefore, data crawled from Twitter is a good source for cyberbullying research.

The Twitter dataset is composed of tweets crawled by the public Twitter stream API. Each tweet contains at least one of the following keywords: bully, bullied, bullying. Retweets are removed by excluding tweets containing the acronym 'RT'. Finally, 1762 tweets are sampled uniformly from the whole tweets collections on August 6, 2011 and

\footnotetext{
${ }^{2}$ The dataset has been kindly provided at http://research.cs.wisc.edu/bullying/data. html

${ }^{3}$ http://news.wisc.edu/20931
} 


\section{Non-Bullying Trace}

1 Don't let your mind bully your body into believing it must carry the burden of its worries. \#TeamFollowBack

2 Whether life's disabilities, left you outcast, bullied or teased, rejoice and love yourself today, 'Cause baby, you were born this way

3 @USERNAME haha hopefully! Beliebers just bring a new meaning to cyber bullying

\section{Bullying Trace}

$1 @$ RodFindlay been sent a few of them. Thought they could bully me about. Put them right and they won't represent the client anymore!

2 He a bully on his block, in his heart he a clown

3 I was bullied \#wheniwas13 but now I am the OFFICE bully!!

Figure 6.3: Some Examples from Twitter Datasets. Three of them are nonbullying traces. And the other three are bullying traces.

manually labeled. It should be pointed out here that labeling is based on bullying traces. Bullying traces are defined as response to the bullying experience, which include but far exceed the incidences of cyberbullying. Some examples of bullying traces are shown in Figure 6.3. To preprocess these tweets, a twitter-specialized tokenizer ${ }^{4}$ is applied without any stemming or stopword removal operations. In addition, some special characters including user mentions, URLS and so on are replaced by predefined characters, respectively. The statistics of this dataset can be found in Table 6.2. Since the dataset does not have explicit train/test split, 5-fold cross validation (CV) is applied, where four-folds are used for training and the remaining one is used for testing. The mean results will be reported.

Experimental Setup

The following methods will be compared:

${ }^{4}$ http://www .ark.cs.cmu.edu/TweetNLP/ 
TABLE 6.2: Statistical properties of the Twitter dataset.

\begin{tabular}{c|c}
\hline Statistics & Twitter \\
\hline Sample No. & 1762 \\
\hline Bullying Instances & 684 \\
\hline Train/Test & 5 -fold \\
\hline
\end{tabular}

* BoW Model: the raw BoW features are directly fed into the classifier.

* Semantic-enhanced BoW Model: This approach is referred in [154]. Following the original setting, we scale the bullying features by a factor of 2 . To give a fair comparison, the bullying features here is insulting seeds used in our proposed method.

* LSA: Latent Semantic Analysis [44].

* LDA: Latent Dirchilet Allocation [102]. Our implementation of LDA is based on Gensim $^{5}$.

* EBoW: Our proposed Embeddings-enhanced Bag-of-Words Model.

For BoW, the most frequent 2000 terms including unigram and bigram are used as features. For LSA and LDA, the number of latent topics are both set to 100. To implement LDA, we set hyperparameter $\alpha$ for document topic multinomial and hyperparameter $\eta$ for word topic multinomial to 1 and 0.01 , respectively.

For our proposed method, BoW and latent semantic features are both the same as those in two above compared models. For bullying features, the number of insulting seeds is 20. The number of similar words compared to each insulting seed $h$ is set to 50 . After feature expanding based on word embeddings, the length of bullying features is 641 finally. Therefore, the dimension of features learned by our EBoW is the sum of the lengths of the three kinds of features as: $2000+100+641=2741$.

We then apply linear SVM [138] in the new feature space generated by the above mentioned approaches. In linear SVM, the hyper-parameter: regularization term $\mathrm{C}$, is searched over a range as $\left\{2^{-2}, 2^{-1}, 2^{0}, 2^{1}, 2^{2}, 2^{3}, 2^{4}, 2^{5}\right\}$ via a five-fold cross-validation conducted on training data.

The cyberbullying detection task is thus converted into a binary classification problem. The adopted evaluation metrics include precision, recall and F1-value.

\footnotetext{
${ }^{5}$ https ://radimrehurek. com/gensim/index.html
} 
TABle 6.3: Precision (\%), Recall (\%) and F1 Scores (\%) for compared methods. Bold face indicates best performance.

\begin{tabular}{c|c|c|c|c|c}
\hline Measures & BoW & sBoW & LSA & LDA & EBoW \\
\hline Precision & 75.6 & 75.7 & 75.9 & 74.0 & $\mathbf{7 6 . 8}$ \\
Recall & 77.8 & 78.3 & 78.2 & 76.5 & $\mathbf{7 9 . 4}$ \\
F1 Score & 76.6 & 76.9 & 77.0 & 74.9 & $\mathbf{7 8 . 0}$ \\
\hline
\end{tabular}

Experimental Results We reported the precision, recall and F1 scores of all compared methods in Table 6.3. Obviously, our proposed method outperforms other compared methods in all the three evaluation metrics.

It is observed that semantic BoW model is able to give a slight better performance than BoW. In sBoW, the weights corresponding to bullying features are multiplied by 2 and the bullying features are the same as the insulting seeds in our proposed EBoW. This indicates that bullying features can indeed improve cyberbullying detection. Different from a direct use of insulting seeds in sBoW, our approach EBoW expands the pre-defined insulting words through word embeddings and assign different weights to these expanded bullying features according to cosine similarity between word embeddings. As a result, our approach is able to gain a significant performance improvement compared to sBoW over all three evaluation measures.

We also observe that our method outperforms two stat-of-arts text representation learning methods: LSA and LDA. This may be because that both methods belong to dimensionality reduction. The reduced dimension is a key parameter to determine the quality of learned feature space. Here, we fix the dimension of latent space to 100. A deliberate searching for this parameter may boost the performances of LSA and LDA

Parameters Sensitivity In our proposed EBoW model, we select the top- $h$ similar words for each pre-defined insulting word. Parameter $h$ is used as the threshold to expand bullying features. An increased $h$ will mean more terms are considered as bullying features so that the dimension of bullying features increases. Here, we investigate the influence of the parameter on our model performance for cyberbullying detection. Parameter $h$ is chosen from a predefined set: $[0,50,100,150,200,250]$. When $h=0$, bullying features only consist of our pre-defined insulting words. According to the total 6 settings of $h$, we feed our corresponding learned EBoW features into the classifier. The other experimental settings are kept unchanged. Then, precision, recall and F1 scores for these different settings are calculated and reported in Figure 6.4.

It shows that a moderate $h$ is able to achieve the best performance. If $h$ is too small, some discriminative terms that are not covered by the pre-defined insulting words are 


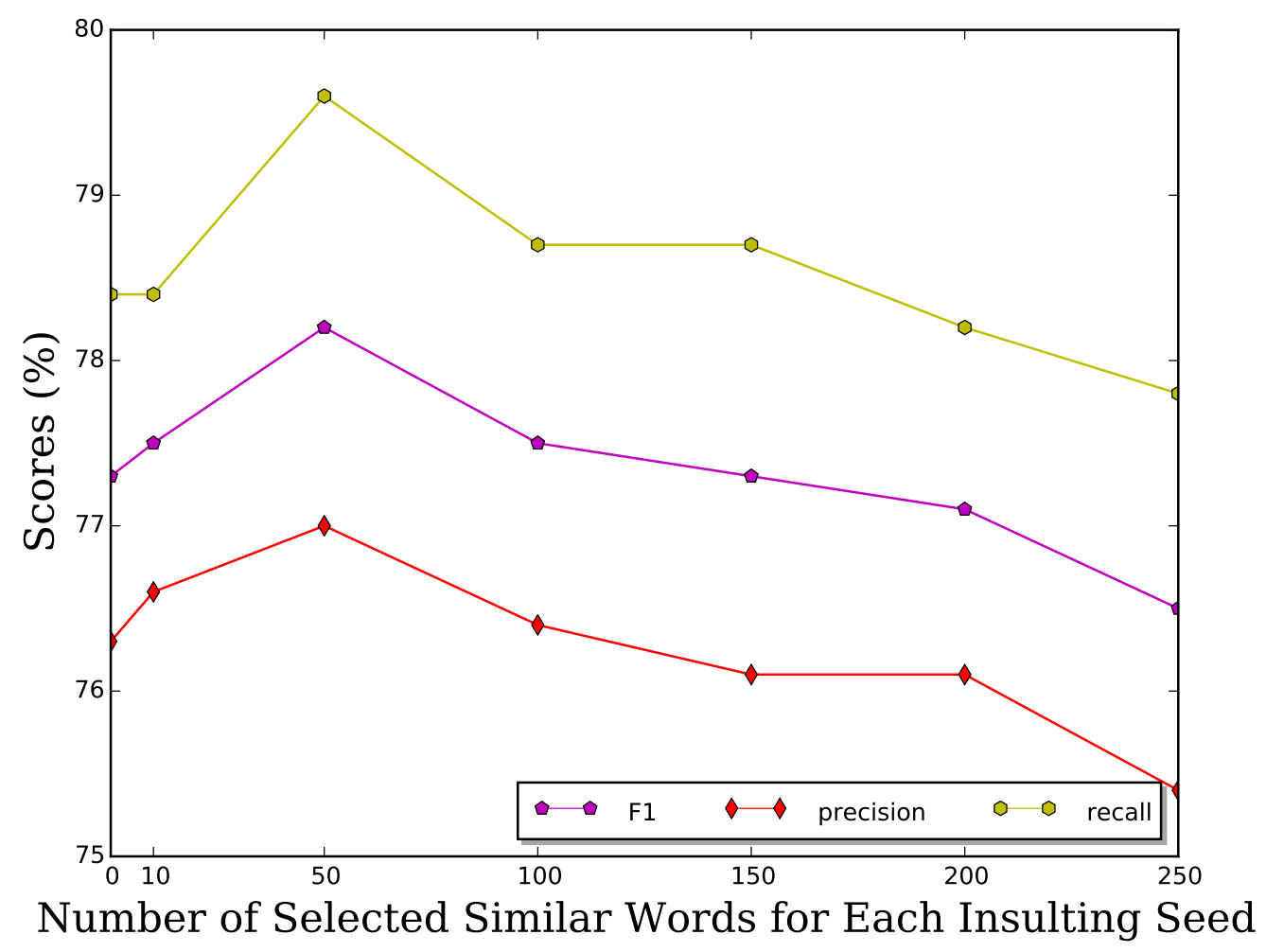

Figure 6.4: Performance of our proposed EBoW model for different $h$. Different colors indicate different measures.

filtered out in the learned bullying features. If $h$ is too large, some irrelevant terms are considered as bullying features. Both scenarios will lead to an ineffective feature space for cyberbullying detection. As a result, a classifier applied on such a feature space may not produce satisfying performance.

\subsection{Semantic-Enhanced Marginalized Stacked De- noising Auto-encoder for Cyberbullying De- tection}

In this section, we investigate one deep learning method named stacked denoising autoencoder (SDA) [79]. SDA stacks several denoising autoencoders and concatenates the output of each layer as the learned representation. Each denoising autoencoder in SDA is trained to recover the input data from a corrupted version of it. The input is corrupted 
by randomly setting some of the input to zero, which is called dropout noise. This denoising process helps the autoencoders to learn robust representation. In addition, each autoencoder layer is intended to learn an increasingly abstract representation of the input [160]. Here, we develop a new text representation model based on a variant of SDA: marginalized stacked denoising autoencoders (mSDA) [88], which adopts linear instead of nonlinear projection to accelerate training and marginalizes infinite noise distribution in order to learn more robust representations. We utilize semantic information to expand mSDA and develop Semantic-enhanced Marginalized Stacked Denoising Autoencoders (smSDA). In our application, the semantic information consists of bullying words. An automatic extraction of bullying words based on word embeddings is proposed so that the involved human labor can be reduced. During training of smSDA, we attempt to reconstruct bullying features from other normal words by discovering the latent structure, i.e. correlation, between bullying and normal words. The intuition behind this idea is that some bullying messages do not contain bullying words. The correlation information discovered by smSDA helps to reconstruct bullying features from normal words, and this in turn facilitates detection of bullying messages without containing bullying words. For example, there is a strong correlation between bullying word fuck and normal word off since they often occur together. If bullying messages do not contain such obvious bullying features, such as fuck is often misspelled as $f c k$, the correlation may help to reconstruct the bullying features from normal ones so that the bullying message can be detected. It should be noted that introducing dropout noise has the effects of enlarging the size of the dataset, including training data size, which helps alleviate the data sparsity problem. In addition, L1 regularization of the projection matrix is added to the objective function of each autoencoder layer in our model to enforce the sparstiy of projection matrix, and this in turn facilitates the discovery of the most relevant terms for reconstructing bullying terms. The main contributions of our work can be summarized as follows:

* Our proposed Semantic-enhanced Marginalized Stacked Denoising Autoencoder is able to learn robust features from BoW representation in an efficient and effective way. These robust features are learned by reconstructing original input from corrupted (i.e., missing) ones. The new feature space can improve the performance of cyberbullying detection even with a small labeled training corpus.

* Semantic information is incorporated into the reconstruction process via the designing of semantic dropout noises and imposing sparsity constraints on mapping matrix. In our framework, high-quality semantic information, i.e., bullying words, can be extracted automatically through word embeddings. Finally, these specialized modifications make the new feature space more discriminative and this in turn facilitates bullying detection. 
* Comprehensive experiments on real-data sets have verified the performance of our proposed model.

We first introduce notations which will be used in the following. Let $D=\left\{w_{1}, \ldots, w_{d}\right\}$ be the dictionary covering all the words existing in the text corpus. We represent each message using a BoW vector $\mathbf{x} \in \mathbb{R}^{d}$. Then, the whole corpus can be denoted as a matrix: $\mathbf{X}=\left[\mathbf{x}_{1}, \ldots, \mathbf{x}_{n}\right] \in \mathbb{R}^{d \times n}$, where $n$ is the number of available posts.

\subsection{1 mSDA: Marginalized Stacked Denoising Auto-encoder}

In this Section, we briefly review the marginalized stacked denoising auto-encoder and present our proposed Semantic-enhanced Marginalized Stacked Denoising Auto-Encoder. Chen et.al proposed a modified version of Stacked Denoising Auto-encoder that employs a linear instead of a non-linear projection so as to obtain a closed-form solution [88]. The basic idea behind denoising auto-encoder is to reconstruct the original input from a corrupted one $\tilde{\mathbf{x}}_{1}, \ldots, \tilde{\mathbf{x}}_{n}$ with the goal of obtaining robust representation.

The basic module behind msDA is Marginalized Denoising Auto-encoder. In this module, denoising auto-encoder attempts to reconstruct original data using the corrupted data via a linear projection. The projection matrix can be learned as:

$$
\mathbf{M}=\underset{\mathbf{M}}{\operatorname{argmin}} \frac{1}{2 n} \sum_{i=1}^{n}\left\|\mathbf{x}_{i}-\mathbf{M} \tilde{\mathbf{x}}_{i}\right\|^{2}
$$

where $\mathbf{M} \in \mathbb{R}^{d \times d}$. For simplicity, we can write Eq. (6.3) in matrix form as:

$$
\mathbf{M}=\underset{\mathbf{M}}{\operatorname{argmin}} \frac{1}{2 n} \operatorname{tr}\left[(\mathbf{X}-\mathbf{M} \tilde{\mathbf{X}})^{\mathrm{T}}(\mathbf{X}-\mathbf{M} \tilde{\mathbf{X}})\right]
$$

where $\tilde{\mathbf{X}}=\left[\tilde{\mathbf{x}}_{1}, \ldots, \tilde{\mathbf{x}}_{n}\right]$ is the corrupted version of $\mathbf{X}$. It is easily shown that Eq. (6.4) is an ordinary least square problem having a closed-form solution:

$$
\mathbf{M}=\mathbf{P Q}^{-1}
$$

where $\mathbf{P}=\mathbf{X} \tilde{\mathbf{X}}^{\mathrm{T}}$ and $\mathbf{Q}=\tilde{\mathbf{X}} \tilde{\mathbf{X}}^{\mathrm{T}}$. In fact, this corruption can be marginalized over the noise distribution [88]. The more corruptions we take in the denoising auto-encoder, the more robust transformation can be learned. Therefore, the best choice is using infinite versions of corrupted data. If the data corpus is corrupted infinite times, the matrix $P$ 
and $Q$ are converged to their corresponding expectation, and Eq. (6.5) can be formulated as:

$$
\mathbf{M}=E[\mathbf{P}] E[\mathbf{Q}]^{-1}
$$

where $E[\mathbf{P}]=\sum_{i=1}^{n} E\left[\mathbf{x}_{i} \tilde{\mathbf{x}}_{i}^{\mathrm{T}}\right]$ and $E[\mathbf{Q}]=\sum_{i=1}^{n} E\left[\tilde{\mathbf{x}}_{i} \tilde{\mathbf{x}}_{i}^{\mathrm{T}}\right]$. These expected matrices can be computed based on noise distribution. In [88], dropout noise is adopted to corrupt data samples by setting a feature to zero with a probability $p$. Assuming the scatter matrix of the original data samples is denoted as $\mathbf{S}=\mathbf{X X}^{\mathrm{T}}$, the expected matrices can be computed as:

$$
E[\mathbf{Q}]_{i, j}= \begin{cases}(1-p)^{2} \mathbf{S}_{i, j} & \text { if } i \neq j, \\ (1-p) \mathbf{S}_{i, j} & \text { if } i=j\end{cases}
$$

and

$$
E[\mathbf{P}]_{i, j}=(1-p) \mathbf{S}_{i, j}
$$

where $i$ and $j$ denotes the indices of features. It can be seen that it is very efficient to compute $\mathbf{M}$ by marginalizing dropout noise in denoising auto-encoder. After the mapping weights $\mathbf{M}$ are computed, a nonlinear squashing function, such as a hyperbolic tangent function, can be applied to derive the output of the marginalized denoising auto-encoder:

$$
\mathbf{H}=\tanh (\mathbf{M X})
$$

To achieve a deep structure, Chen et.al [88] also proposed to apply stacking structures on marginalized denoising autoencoder, in which the output of the $(k-1)^{t h}$ layer is fed as the input into the $k^{t h}$ layer. If we define the output of the $k^{t h} \mathrm{mDA}$ as $\mathbf{H}_{k}$ and the original input as $\mathbf{H}_{0}$ respectively, the mapping between two consecutive layers is given as:

$$
\mathbf{H}_{k}=\tanh \left(\mathbf{M}_{k} \mathbf{H}_{k-1}\right)
$$

where $\mathbf{M}_{k}$ denotes the mapping in $k^{t h}$ layer. The model training can be done greedily layer by layer. This means that the mapping weights $\mathbf{M}_{k}$ is learned in a closed-form to reconstruct the output of $(k-1)^{t h} \mathrm{mDA}$ layer from its marginalized corruptions, as shown in Eq. (6.6). If the number of layers is set to $L$, the final representation for input data $\mathbf{X}$ is the concatenation of the uncorrupted original input and outputs of all layers as follows: 


$$
\mathbf{Z}=\left[\begin{array}{c}
\mathbf{X} \\
\mathbf{H}_{1} \\
\vdots \\
\mathbf{H}_{L}
\end{array}\right]
$$

where $\mathbf{Z} \in \mathbb{R}^{d(L+1) \times n}$. Each column of $\mathbf{Z}$ represents the final representation of each individual data sample.

\subsection{2 smSDA: Semantic-Enhanced Marginalized Stacked De- noising Auto-encoder}

The advantage of corrupting the original input in mSDA can be explained by feature co-occurrence statistics. The co-occurrence information is able to derive a robust feature representation under an unsupervised learning framework, and this also motivates other state-of-the-art text feature learning methods such as Latent Semantic Analysis and topic models [44, 102]. As shown in Figure 6.5. (a), a denoising auto-encoder is trained to reconstruct these removed features values from the rest uncorrupted ones. Thus, the learned mapping matrix $W$ is able to capture correlation between these removed features and other features. It is shown that the learned representation is robust and can be regarded as a high level concept feature since the correlation information is invariant to domain-specific vocabularies. We next describe how to extend mSDA for cyberbullying detection. The major modifications include semantic droupout noise and sparse mapping constraints.

\section{Semantic Dropout Noise}

The dropout noise adopted in mSDA is an uniform distribution, where each feature has the same probability to be removed. In cyberbullying detection, most bullying posts contain bullying words such as profanity words and foul languages. These bullying words are very predictive of the existence of cyberbullying. However, a direct use of these bullying features may not achieve good performance because these words only account for a small portion of the whole vocabulary and these vulgar words are only one kind of discriminative features for bullying $[152,155]$. In other way, we can explore these cyberbullying words by using a different dropout noise that features corresponding to bullying words have a larger probability of corruption than other features. The imposed large probability on bullying words emphasizes the correlation between bullying features 
and normal ones. This kind of dropout noise can be denoted as semantic dropout noise, because semantic information is used to design dropout structure.

As shown in Figure 6.5. (b), the correlation between features can enable other normal words to predict bullying labels. Considering a simple but intuitive example, "Leave him alone, he is just a chink" ${ }^{6}$, which is obviously a bullying message. However, the classifier will set the weight of the discriminative word "chink" to zero, if the small sized training corpus does not cover it. Our proposed smSDA can deal with the problem by learning a robust feature representation, which is a high level concept representation. In the learned representation, the word "chink" are reconstructed by context words cooccurring with the specific word ("chink") and the context words may be shared by other bullying words contained in training corpus. Therefore, the correlation explored by this auto-encoder structure enables the subsequent classifier to learn the discriminative word and improve the classification performance. In addition, the semantic dropout noise exploits the correlation between bullying features and normal features better and hence, facilitates cyberbullying detection.

Due to the introduced semantic dropout noise, the expected matrices: $E[\mathbf{P}]$ and $E[\mathbf{Q}]$ will be computed slightly different from Eqs. (6.7) and (6.8). Assuming we have an available bullying words list and the corresponding features set $\mathbb{Z}_{b}$, the semantic dropout noise can be described as the following probability density function (PDF):

$$
P D F= \begin{cases}p\left(\tilde{x}_{d}=0\right)=p_{n} & \text { if } d \notin \mathbb{Z}_{b}, \\ p\left(\tilde{x}_{d}=x_{d}\right)=1-p_{n} & \text { if } d \notin \mathbb{Z}_{b}, \\ p\left(\tilde{x}_{d}=0\right)=p_{b} & \text { if } d \in \mathbb{Z}_{b}, \\ p\left(\tilde{x}_{d}=x_{d}\right)=1-p_{b} & \text { if } d \in \mathbb{Z}_{b},\end{cases}
$$

where $d$ denotes the feature set. Then these two marginalized matrices can be computed as:

$6 "$ Chink (also chinki, chinky, chinkie) is an English ethnic slur usually referring to a person of Chinese or East Asian ethnicity" from Wikipedia 


$$
\begin{aligned}
& E[\mathbf{Q}]_{i, j}= \\
& \begin{cases}\left(1-p_{n}\right) \mathbf{S}_{i, j} & \text { if } i=j \& i \notin \mathbb{Z}_{b}, \\
\left(1-p_{n}\right)^{2} \mathbf{S}_{i, j} & \text { if } i \neq j \&\{i, j\} \cap \mathbb{Z}_{b}=\varnothing, \\
\left(1-p_{b}\right)\left(1-p_{n}\right) \mathbf{S}_{i, j} & \text { if }\{i, j\} \notin \mathbb{Z}_{b} \&\{i, j\} \cap \mathbb{Z}_{b} \neq \varnothing, \\
\left(1-p_{b}\right)^{2} \mathbf{S}_{i, j} & \text { if } i \neq j \&\{i, j\} \in \mathbb{Z}_{b}, \\
\left(1-p_{b}\right) \mathbf{S}_{i, j} & \text { if } i=j \& i \in \mathbb{Z}_{b} .\end{cases}
\end{aligned}
$$

and

$$
E[\mathbf{P}]_{i, j}= \begin{cases}\left(1-p_{n}\right) \mathbf{S}_{i, j} & \text { if } j \cap \mathbb{Z}_{b}=\varnothing, \\ \left(1-p_{b}\right) \mathbf{S}_{i, j} & \text { if } j \cap \mathbb{Z}_{b} \neq \varnothing .\end{cases}
$$

where $p_{b}$ and $p_{n}$ are the probabilities of bullying features and normal features to be set to zero respectively, and $p_{b}>p_{n}$. Here, $p_{b}$ and $p_{n}$ are both tunable hyperparameters for our proposed smSDA.

Unbiased Semantic Dropout Noise As shown in Eq . (6.12), the corrupted data is biased, i.e., $E[\mathbf{X}] \neq E[\tilde{\mathbf{X}}]$. Here, we modified Eq. (6.12) to achieve an unbiased noise as follows:

$$
\text { PDF } F^{\text {unbiased }}= \begin{cases}p\left(\tilde{x}_{d}=0\right)=p_{n} & \text { if } d \notin \mathbb{Z}_{b}, \\ p\left(\tilde{x}_{d}=\frac{x_{d}}{1-p_{n}}\right)=1-p_{n} & \text { if } d \notin \mathbb{Z}_{b}, \\ p\left(\tilde{x}_{d}=0\right)=p_{b} & \text { if } d \in \mathbb{Z}_{b}, \\ p\left(\tilde{x}_{d}=\frac{x_{d}}{1-p_{b}}\right)=1-p_{b} & \text { if } d \in \mathbb{Z}_{b},\end{cases}
$$

It can be easily shown that under such a noise distribution, the corrupted data is unbiased now. These two marginalized matrices are re-formulated as:

$$
E[\mathbf{Q}]_{i, j}^{\text {unbiased }}= \begin{cases}\frac{1}{1-p_{n}} \mathbf{S}_{i, j} & \text { if } i=j \& i \notin \mathbb{Z}_{b} \\ \frac{1}{1-p_{b}} \mathbf{S}_{i, j} & \text { if } i=j \& i \in \mathbb{Z}_{b} \\ \mathbf{S}_{i, j} & \text { if } i \neq j .\end{cases}
$$

and

$$
E[\mathbf{P}]_{i, j}^{\text {unbiased }}=\mathbf{S}_{i, j}
$$

These two computed matrices will then be used to learn the mapping in each layer in our proposed smSDA. 
Sparsity Constraints In mSDA, the mapping matrix $\mathbf{M}$ is learned to reconstruct removed features from other uncorrupted features and hence is able to capture the feature correlation information. Here, we inject the sparsity constraints on the mapping weights $\mathbf{M}$ so that each row has a small number of nonzero elements. This sparsity constraint is quite intuitive because one word is only related to a small portion of vocabulary instead of the whole vocabulary. In our proposed smSDA, the sparsity constraint is realized by the incorporation of $\mathrm{L} 1$ regularization term into the objective function as in the lasso problem [87]. The optimization function for each layer in smSDA is given as follows:

$$
\mathbf{M}=\underset{\mathbf{M}}{\operatorname{argmin}} \frac{1}{2 n} \operatorname{tr}\left[(\mathbf{X}-\mathbf{M} \tilde{\mathbf{X}})^{\mathrm{T}}(\mathbf{X}-\mathbf{M} \tilde{\mathbf{X}})\right]+\lambda\|\mathbf{M}\|_{1}
$$

where $\lambda$ is a regularization parameter that controls the sparsity of $\mathbf{M}$. The larger the $\lambda$ is, the sparser the mapping matrix $\mathbf{M}$ is. The solution to Eq. (6.18) is a very mature math problem: sparse least squares optimization, which has several effective and efficient computation methods [161-163]. Here, we adopt a method called Iterated Ridge Regression, which has been proven to be very efficient [163]. The method firstly introduces an approximation:

$$
\left\|\mathbf{w}_{i}\right\|_{1} \approx \frac{\mathbf{w}_{i}^{\mathrm{T}} \mathbf{w}_{i}}{\left\|\mathbf{w}_{i}\right\|_{1}}
$$

where $\mathbf{w}_{i}$ denotes the $i$-th row in the whole matrix $\mathbf{M}$. By substituting this approximation Eq. (6.19) into the objective function Eq. (6.18), we yield an formulation similar to a Ridge Regression Problem [164], and the iteration steps to solve $\mathbf{M}$ is given as:

$$
\mathbf{M}_{k}=\tilde{\mathbf{X}}^{\mathrm{T}} \mathbf{X}\left[\tilde{\mathbf{X}}^{\mathrm{T}} \tilde{\mathbf{X}}+\lambda \operatorname{diag}\left(\left|\mathbf{M}_{k-1}\right|\right)^{-1}\right]^{-1}
$$

where diag denotes the diagonal elements of a matrix, $\mathbf{M}_{k}$ and $\mathbf{M}_{k-1}$ denote the current step and the previous step estimations for mapping matrix $\mathbf{M}$, respectively. It is clear that the Eq. (6.20) can be easily formulated when the noise distribution is marginalized. Similar to Eq. (6.6), Eq. (6.20) can be written as:

$$
\mathbf{M}_{k}=E[\mathbf{P}]\left[E[\mathbf{Q}]+\lambda \operatorname{diag}\left(\left|\mathbf{M}_{k-1}\right|\right)^{-1}\right]^{-1}
$$

To speed up the convergence process, the initialization for $\mathbf{M}$ can be set to the L2 penalized solution for Eq. (6.4) as follows:

$$
\mathbf{M}_{0}=E[\mathbf{P}][E[\mathbf{Q}]+\lambda \mathbf{I}]^{-1}
$$




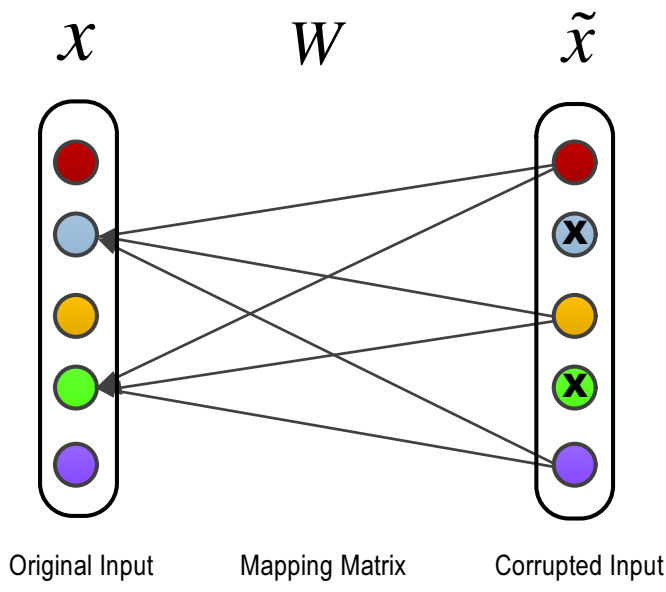

(a)

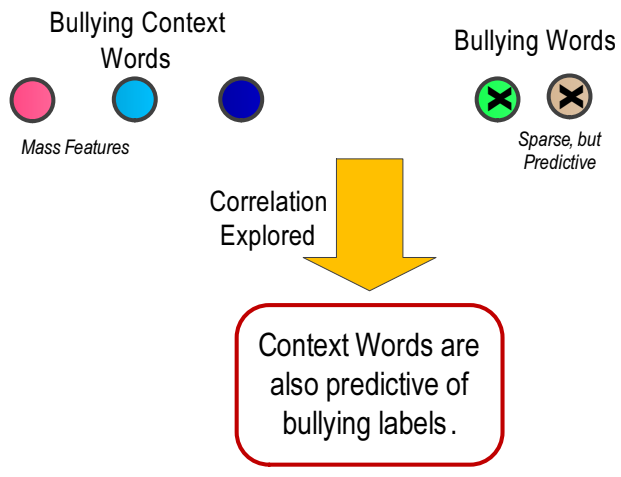

(b)

FiguRE 6.5: Illustration of Motivations behind smSDA. In Figure 1(a), the cross symbol denotes that its corresponding feature is corrupted, i.e., turned off.

where $\mathbf{I}$ is an identify matrix. It can be shown that this iteration procedure can also marginalize the noise distribution easily, which can ensure an efficient and stable mapping learning.

Construction of Bullying Feature Set As analyzed above, the bullying features play an important role and should be chosen properly. In the following, the steps for constructing bullying feature set $\mathbb{Z}_{b}$ are given, in which the first layer and the other layers are addressed separately. For the first layer, expert knowledge and word embeddings are used. For the other layers, discriminative feature selection is conducted.

For layer one, we firstly build a list of words with negative affective, including swear words and dirty words. Then, we compare the word list with the BoW features of our own corpus, and regard the intersections as bullying features. However, it is possible that expert knowledge is limited and does not reflect the current usage and style of cyberlanguage. Therefore, we expand the list of pre-defined insulting words, i.e. insulting seeds, based on word embeddings as follows:

Word embeddings use real-valued and low-dimensional vectors to represent semantics of words $[19,53]$. The well-trained word embeddings lie in a vector space where similar words are placed close to each other. In addition, the cosine similarity between word embeddings is able to quantify the semantic similarity between words. Considering the Interent messages are our interested corpus, we utilize a well-trained word2vec model on a large-scale twitter corpus containing 400 million tweets [159]. A visualization of some word embeddings after dimensionality reduction (PCA) is shown in Figure 6.2. Here, we adopt PCA to reduce the dimensionality of several words into two and visualize 
them. It is observed that curse words form distinct clusters, which are also far away from normal words. Even insulting words are located at different regions due to different word usages and insulting expressions. In addition, since the word embeddings adopted here are trained in a large scale corpus from Twitter, the similarity captured by word embeddings can represent the specific language pattern. For example, the embedding of the misspelled word $f c k$ is close to the embedding of $f u c k$ so that the word $f c k$ can be automatically extracted based on word embeddings.

We extend the pre-defined insulting seeds based on word embeddings. For each insulting seed, similar words are extracted if their cosine similarities with insult seed exceed a predefined threshold. For bigram $w_{l} w_{r}$, we simply use an additive model to derive the corresponding embedding as follows:

$$
\mathbf{v}\left(w_{l} w_{r}\right)=\mathbf{v}\left(w_{l}\right)+\mathbf{v}\left(w_{r}\right)
$$

Finally, the constructed bullying features are used to train the first layer in our proposed smSDA. It includes two parts: one is the original insulting seeds based on domain knowledge and the other is the extended bullying words via word embeddings. The length of $\mathbb{Z}_{b}$ is $k$.

For subsequent layers, we perform feature selection using Fisher score to select 'bullying"' features. Fisher score is an univariate metric reflecting the discriminative power of a feature $[165,166]$. For the $r^{\text {th }}$ feature, the corresponding Fisher score can be computed based on training data with labels:

$$
F_{r}=\frac{\sum_{i=1}^{c} n_{i}\left(\mu_{i}-\mu\right)^{2}}{\sum_{i=1}^{c} n_{i} \sigma_{i}{ }^{2}}
$$

where $c$ denotes the number of classes and $n_{i}$ represent the number of data in class $i . \mu$ and $\mu_{i}$ denote the mean of entire data and class $i$ for the $r^{\text {th }}$ feature, and $\sigma_{i}$ is the variance of class $i$ on $r^{\text {th }}$ feature. After Fisher scores are estimated, features with top $k$ scores are selected as "bullying" features, where "bullying" is generalized as discriminative.

\subsection{3 smSDA for Cyberbullying Detection}

After the introduction of the Semantic-enhanced Marginalized Stacked Denoising Autoencoder (smSDA), we describe how to leverage it for cyberbullying detection. smSDA provides robust and discriminative representations The learned numerical representations 
can then be fed into Support Vector Machine (SVM). In the new space, due to the captured feature correlation and semantic information, the SVM, even trained in a small size of training corpus, is able to achieve a good performance on testing documents (this will be verified in the following experiments). The detailed steps of our model are provided below:

Assuming the first $n_{l}$ posts are labeled and the corresponding vector of binary labels is $\mathbf{y}=\left\{y_{1}, \ldots, y_{n_{l}}\right\}$. The binary label 1 or 0 indicates the post is or is not a cyberbullying one. Here, $n_{l} \ll n$, which means the labeled posts have a small size. The bullying feature set $\mathbb{Z}_{b}$ is constructed in a layer-wise way. Based on prior knowledge, we construct a predefined bullying wordlist and compare it with the original vocabulary of the whole corpus $\mathbf{X}$. The words appearing in both the vocabulary and the bullying wordlist are selected as insulting seeds. The insulting seeds are then expanded and refined automatically via word embeddings, which defines the bullying features $\mathbb{Z}_{b}$ for layer one. The following experiments will show that the construction of the set $\mathbb{Z}_{b}$ is very simple and efficient with litter human labor. For the subsequent layers, after obtaining the output of each layer, the set $\mathbb{Z}_{b}$ is updated using feature ranking with Fish score according to Eq. (6.24).

Based on predefined dropout probabilities for bullying features and other normal features $p_{b}$ and $p_{n}$ and the bullying feature set $\mathbb{Z}_{b}$, we compute these two expected matrices $E[\mathbf{P}]$ and $E[\mathbf{Q}]$ according to Eqs. (6.14) and (6.13), if the semantic dropout noise is adopted. When it comes to the unbiased semantic dropout noise, Eqs. (6.16) and (6.17) instead of Eqs. (6.14) and (6.13) are used to compute these two expected matrices. Then, we iteratively perform Eq. (6.21) for $T_{\max }$ times, where the initial value for $\mathbf{M}$ is calculated based on Eq. (6.22). When the mapping matrix is learned, the output of each layer is given according to Eq. (6.10). Due to the stacking structure, the output of $L$ layers and the initial input are concatenated together to form the final representation $\mathbf{Z} \in \mathbb{R}^{d(L+1) \times n}$ following Eq. (6.11). It is clear that the new space has a dimension of $(L+1) d$. A linear SVM [138] is trained on the training corpus, i.e. the first $n_{l}$ columns in $\mathbf{Z}$ and tested on the rest data samples.

\subsubsection{Merits of smSDA}

Some important merits of our proposed approach are summarized as follows:

1. Most cyberbullying detection methods rely on the BoW model. Due to the sparsity problems of both data and features, the classifier may not be trained very well. Stacked densoing autoencoder (SDA), as an unsupervised representation learning 
method, is able to learn a robust feature space. In SDA, the feature correlation is explored by the reconstruction of corrupted data. The learned robust feature representation can then boost the training of classifier and finally improve the classification accuracy. In addition, the corruption of data in SDA actually generates artificial data to expand data size, which alleviate the small size problem of training data.

2. For cyberbullying problem, we design semantic dropout noise to emphasize bullying features in the new feature space, and the yielded new representation is thus more discriminative for cyberbullying detection.

3. The sparsity constraint is injected into the solution of mapping matrix $\mathbf{M}$ for each layer, considering each word is only correlated to a small portion of the whole vocabulary. We formulate the solution for the mapping weights $\mathbf{M}$ as an Iterated Ridge Regression problem, in which the semantic dropout noise distribution can be easily marginalized to ensure the efficient training of our proposed smSDA.

4. Based on word embeddings, bullying features can be extracted automatically. In addition, the possible limitation of expert knowledge can be alleviated by the use of word embedding.

\subsubsection{Experiments}

In following, we evaluate our proposed semantic-enhanced marginalized stacked denoising auto-encoder (smSDA) with two public real-world cyberbullying corpora. We start by describing the adopted corpora and experimental setup. Experimental results are then compared with other baseline methods to test the performance of our approach. At last, we provide a detailed analysis to explain the good performance of our method.

Descriptions of Datasets Two datasets are used here. One is from Twitter and another is from MySpace groups. The details of these two datasets are described below:

Twitter Dataset: Twitter is "a real-time information network that connects you to the latest stories, ideas, opinions and news about what you find interesting" (https:// about.twitter.com/). Registered users can read and post tweets, which are defined as the messages posted on Twitter with a maximum length of 140 characters.

The Twitter dataset is composed of tweets crawled by the public Twitter stream API through two steps. In Step 1, keywords starting with "bull" including "bully", "bullied" and "bullying" are used as queries in Twitter to preselect some tweets that potentially 
contain bullying contents. Re-tweets are removed by excluding tweets containing the acronym 'RT'. In Step 2, the selected tweets are manually labeled as bullying trace or non-bullying trace based on the contents of the tweets. 7321 tweets are randomly sampled from the whole tweets collections from August 6, 2011 to August 31, 2011 and manually labeled $^{7}$. It should be pointed out here that labeling is based on bullying traces. A bullying trace is defined as the response of participants to their bullying experience. Bullying traces include not only messages about direct bullying attack, but also messages about reporting a bullying experience, revealing self as a victim et. al. Therefore, bullying traces far exceed the incidents of cyberbullying. Automatic detection of bullying traces are valuable for cyberbullying research [167]. Some examples of bullying traces are shown in Figure 6.3. To preprocess these tweets, a tokenizer is applied without any stemming or stopword removal operations. In addition, some special characters including user mentions, URLS and so on are replaced by predefined characters, respectively. The features are composed of unigrams and bigrams that should appear at least twice and the details of preprocessing can be found in [147]. The statistics of this dataset can be found in Table 6.4.

MySpace Dataset: MySpace is another web2.0 social networking website. The registered accounts are allowed to view pictures, read chat and check other peoples' profile information.

The MySpace dataset is crawled from MySpace groups. Each group consists of several posts by different users, which can be regarded as a conversation about one topic. Due to the interactive nature behind cyberbullying, each data sample is defined as a window of 10 consecutive posts and the windows are moved one post by one post so that we got multiple windows [168]. Then, three people labeled the data for the existence of bullying content independently. To be objective, an instance is labeled as cyberbullying only if at least 2 out of 3 coders identify bullying content in the windows of posts. The raw text for these data, as XML files, have been kindly provided by Kontostathis et.al ${ }^{8}$. The XML files contain information about the posts, such as post text, post data, and users' information, which are put into 11 packets. Some posts in MySpace are shown in Figure 6.6. Here, we focus on content-based mining, and hence, we only extract and preprocess the posts' text. The preprocessing steps of the MySpace raw text include tokenization, deletion of punctuation and special characters. The unigrams and bigrams features are adopted here. The threshold for negligible low-frequency terms is set to 20, considering

\footnotetext{
${ }^{7}$ The dataset: bullyingV3.0, has been kindly provided at http://research.cs.wisc.edu/ bullying/data.html

${ }^{8}$ The dataset: MySpace Group, has been kindly provided at http://www.chatcoder.com/ DataDownload
} 
TABLE 6.4: Statistical Properties of the two datasets.

\begin{tabular}{c|c|c}
\hline Statistics & Twitter & MySpace \\
\hline Feature No. & 4413 & 4240 \\
\hline Sample No. & 7321 & 1539 \\
\hline Bullying Instances & 2102 & 398 \\
\hline
\end{tabular}

one post occurred in a long conversation will occur in at least ten windows. The details of this dataset is shown in Table 6.4.

Since there were no standard splits of training vs. test datasets in our adopted Twitter and MySpace corpora, we need to define the training and testing datasets. As analyzed above that the lack of labeled training corpus hinders the development of automatic cyberbullying detection, the sizes of training corpus are all controlled to be very small in our experiments. For Twitter dataset, we randomly select 800 instances, which accounts for $12 \%$ of the whole corpus, as the training data and the rest data samples are used as testing data. To reduce variance, the process is repeated ten times so that we can have ten sub-datasets from Twitter data. For MySpace dataset, we also randomly pick 400 data samples as the training corpus and use the rest data for testing. The process is repeated ten times to generate ten sub-datasets constructed from MySpace data. Finally, we have twenty sub-datasets, in which ten datasets are from Twitter corpus and another ten datasets are from MySpace corpus.

Experimental Setup Here, we experimentally evaluate our smSDA on two cyberbullying detection corpora. The following methods will be compared.

* BWM: Bullying word matching. If the message contains at least one of our defined bullying words, it will be classified as bullying.

* BoW Model: the raw BoW features are directly fed into the classifier.

* Semantic-enhanced BoW Model: This approach is referred in [154]. Following the original setting, we scale the bullying features by a factor of 2 .

* LSA: Latent Semantic Analysis [44].

* LDA: Latent Dirchilet Allocation [102]. Our implementation of LDA is based on Gensim $^{9}$.

* mSDA: marginalized stacked denoising autoencoder [88].

\footnotetext{
${ }^{9}$ https://radimrehurek.com/gensim/index.html
} 
:P: He lasted 30 seconds then acted like he couldn't get up.........UUUU: yea

B_P: And a girly man like you wouldn't last 10 seconds.

P: Heath was ok... I thought Jack Nicholson was a really good Joker :though.

:B_P: I don't know what the big deal was about the Dark Knight, :batman's voice was stupid and over done and heath ledger did a horrible job. Im glad he died. Nothing beats Jack Nickolson's performance of the Joker

Figure 6.6: Some Examples from MySpace Datasets. Two Conversions are Displayed and each one includes a normal post $(P)$ and a bullying post $\left(B_{-} P\right)$.

* smSDA and $\operatorname{smSDA}_{\mathrm{u}}$ : semantic-enhanced marginalized denoising autoencoder that utilizes semantic dropout noise and unbiased one, respectively.

For LSA and LDA, the number of latent topics are both set to 100. In LDA, we set hyperparameter $\alpha$ for document topic multinomial and hyperparameter $\eta$ for word topic multinomial to 1 and 0.01 , respectively. For $\mathrm{mSDA}^{10}$, the noise intensity is set to 0.5 and the number of layers for Tweets and MySpace datasets are both set to 2. Here, the number of layers is only set to be a moderate number instead of a large one, considering a large final dimension will impose a computational burden on the subsequent classifier training.

For our proposed methods including smSDA and $\operatorname{smSDA}_{\mathrm{u}}$ : the noise intensity and the number of layers are set to the same values as in mSDA to give a fair comparison. The bullying noise intensity is set to 0.8 , which is larger than 0.5 . The hyperparameters $\lambda$

\footnotetext{
${ }^{10}$ The code has been kindly provided at http://research.cs.wisc.edu/bullying/data. html
} 


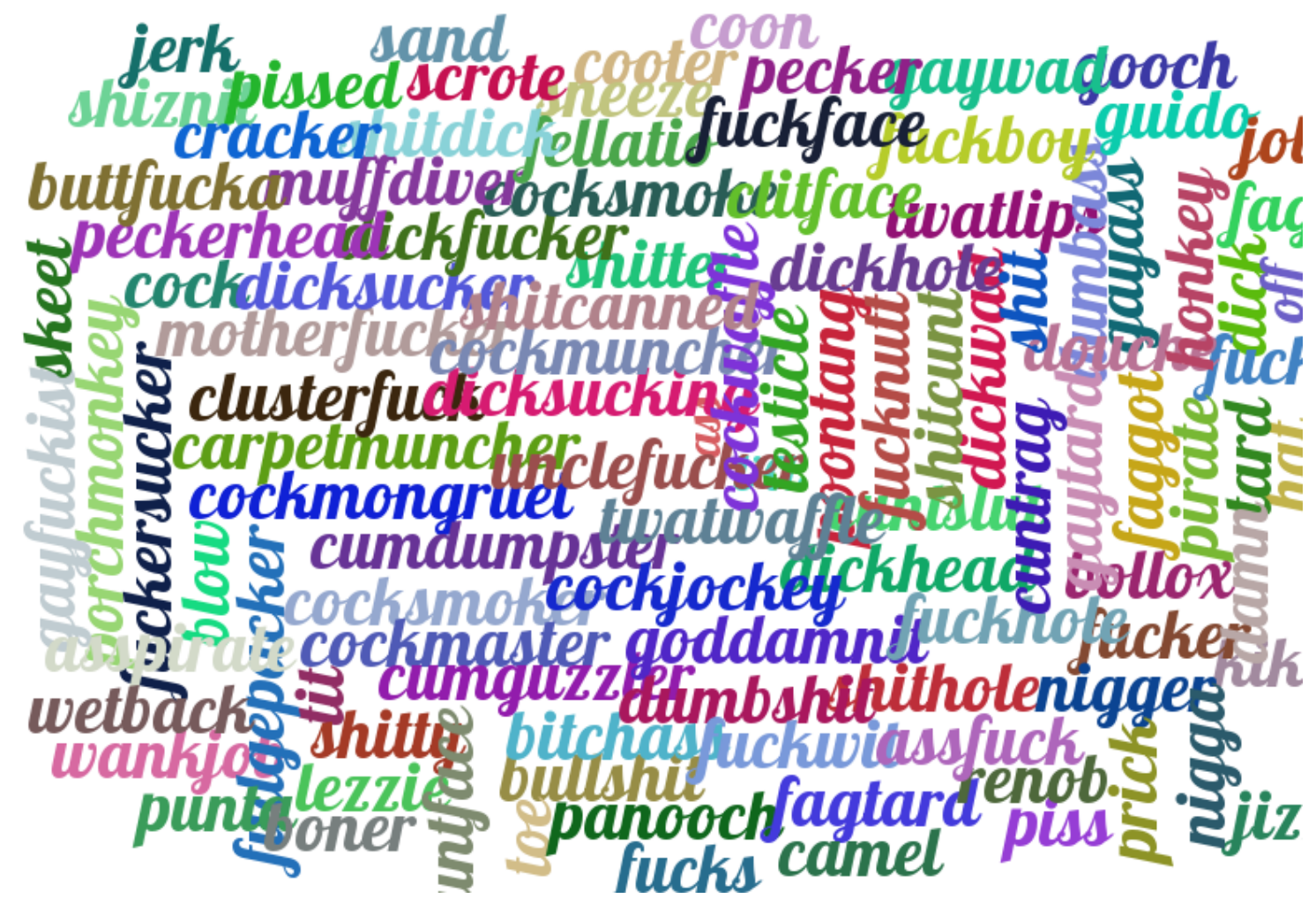

Figure 6.7: Word Cloud Visualization of the List of Words with Negative Affective.

that controls the sparsity of the transformation matrix are set to 1 for all layers. The number of iteration step for solving lasso problems is set to 20 . To construct the bullying features $\mathbb{Z}_{b}$ for the first layer, the negative word list containing 350 words is crawled ${ }^{11}$, whose word cloud visualization is shown in Figure 6.7. The intersections between BoW features of our own corpus and the predefined bullying word list are firstly obtained. Then, as described in 6.3.2, they are extended and refined based on word embeddings to form the final bullying features. The threshold for cosine similarity is set to 0.8 . The word cloud visualizations for the final bullying features in Twitter and MySpace datasets are shown in Figures 6.8 and 6.9, respectively. The bullying features used in Semantic-enhanced BoW Model are the same as those in smSDA.

Linear SVM [138] is then applied to the new feature space generated by the above mentioned approaches. In linear SVM, we search the best regularization parameter C from $\{0.0001,0.001,0.01,0.1,1\}$. To evaluate the performance of these methods on binary classification, classification accuracy is employed. Considering both datasets have the class imbalance problem, we also introduce F1-Score, which is a balance between precision and recall, to evaluate the performance of all compared approaches.

\footnotetext{
${ }^{11} \mathrm{~A}$ collection of insulting words can be found in the website: http://www.noswearing.com/ dictionary
} 


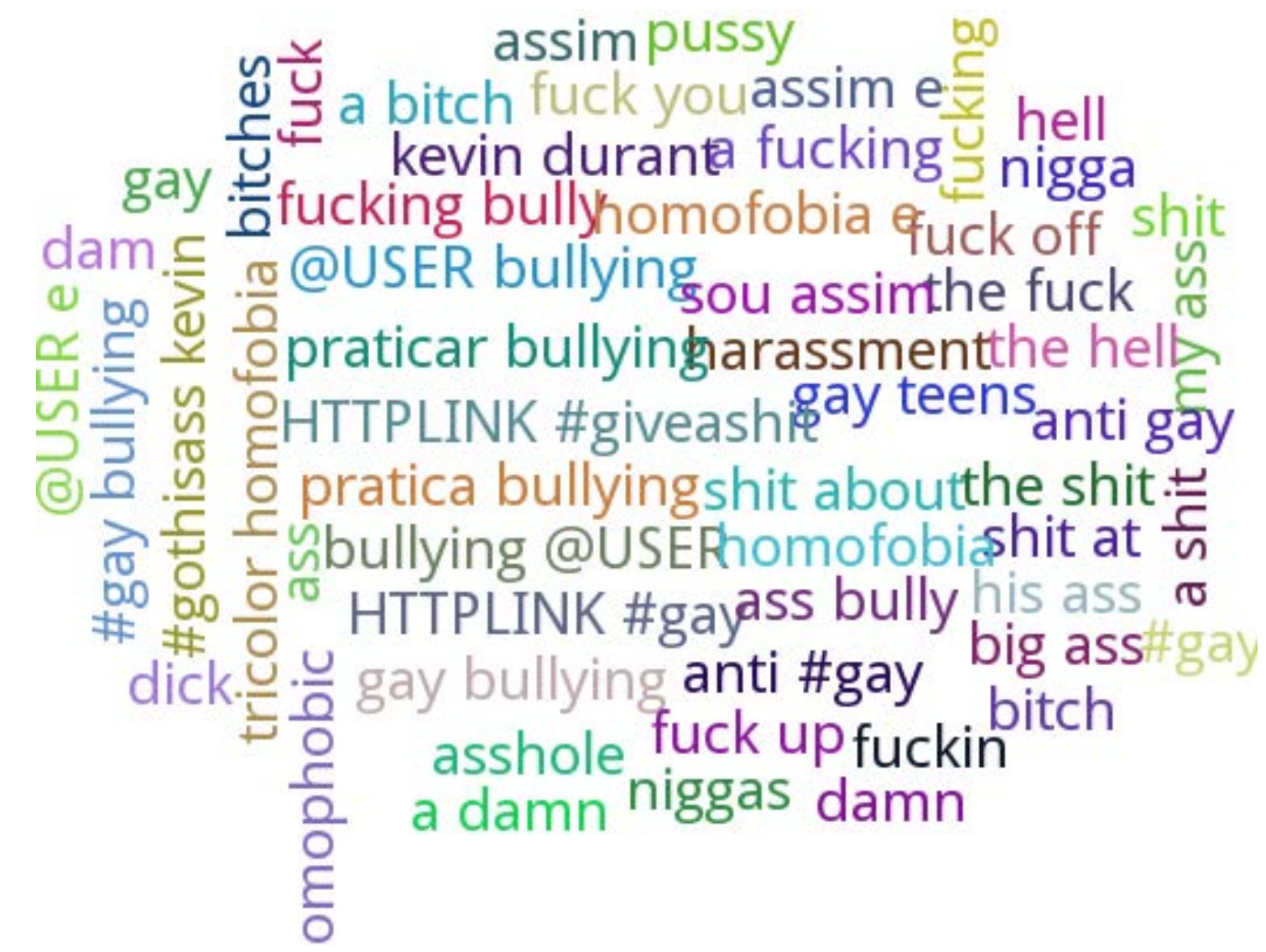

Figure 6.8: Word Cloud Visualization of the Bullying Features in Twitter Datasets.

Experimental Results Here, we show a comparison of our proposed smSDA method with six benchmark approaches on Twitter and MySpace datasets. The average results, for these two datasets, on classification accuracy and F1 score are shown in Table 6.5. Figures 6.10 and 6.11 show the results of seven compared approaches on all sub-datasets constructed from Twitter and MySpace datasets, respectively. Since BWM does not require training documents, its results over the whole corpus are reported in Tables 6.5 and 6.6. It is clear that our approaches outperform the other approaches in these two Twitter and MySpace corpora.

The first observation is that semantic BoW model (sBow) performs slightly better than BoW. Based on BoW, sBoW just arbitrarily scale the bullying features by a factor of 2. This means that semantic information can boost the performance of cyberbullying detection. For a fair comparison, the bullying features used in our method and sBoW are unified to be the same. Our approaches, especially smSDA, gains a significant performance improvement compared to sBoW. This is because bullying features only account for a small portion of all features used. It is difficult to learn robust features for small training data by intensifying each bullying features' amplitude. Our approach aims to 


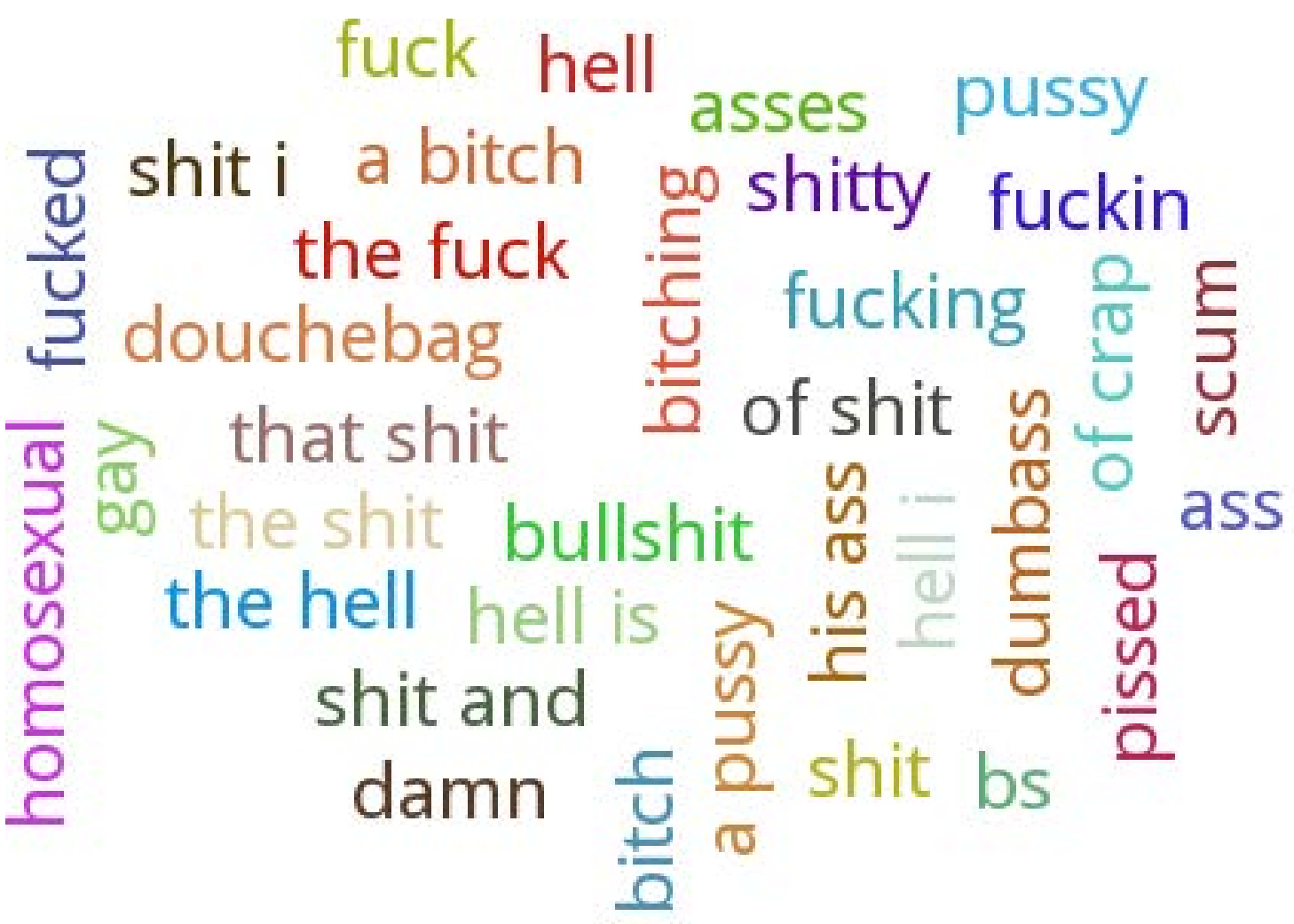

Figure 6.9: Word Cloud Visualization of the Bullying Features in MySpace Datasets.

find the correlation between normal features and bullying features by reconstructing corrupted data so as to yield robust features. In addition, Bullying Word Matching (BWM), as a simple and intuitive method of using semantic information, gives the worst performance. In BWM, the existence of bullying words are defined as rules for classification. It shows that only an elaborated utilization of such bullying words instead of a simple one can help cyberbullying detection.

We also compare our methods with two stat-of-arts text representation learning methods LSA and LDA. These two methods do not produce good performance on all datasets. This may be because that both methods belong to dimensionality reduction techniques, which are performed on the document-word occurrence matrix. Although the two methods try to minimize the reconstruction error as our approach does, the optimization in LSA and LDA is conducted after dimensionality reduction. The reduced dimension is a key parameter to determine the quality of learned feature space. Here, we fix the dimension of latent space to 100. Therefore, a deliberate searching for this parameter which may improve the performances of LSA and LDA and the selection of hyperparameter itself is another tough research topic. Another reason may be that the data samples are small (less than 2000) and the length of each Internet message is short (For Twitter, 
TABle 6.5: Accuracies (\%) for Compared Methods on Twitter and MySpace Datasets. The Mean Values are Given, respectively. Bold Face Indicates Best Performance.

\begin{tabular}{c||c|c}
\hline Dataset & Twitter & MySpace \\
\hline BWM & 69.3 & 34.2 \\
\hline BoW & 82.6 & 80.1 \\
\hline sBoW & 82.7 & 80.1 \\
\hline LSA & 81.6 & 77.7 \\
\hline LDA & 81.1 & 77.8 \\
\hline mSDA & 84.1 & 87.8 \\
\hline smSDA $_{u}$ & 82.9 & 88.0 \\
\hline smSDA & $\mathbf{8 4 . 9}$ & $\mathbf{8 9 . 7}$ \\
\hline
\end{tabular}

maximum length is 140 characters), and thus the constructed document-word occurrence matrix may not represent the true co-occurrence of terms.

Deep learning methods including mSDA and smSDA generally outperform other standard approaches. This trend is particularly prominent in F1 measure because cyberbullying detection problems are class-imbalance. The larger improvements on F1 score verify the performance of our approach further. Deep learning models have achieved remarkable performance in various scenarios with its own robust feature learning ability [5]. mSDA is able to capture the correlation between input features and combine the correlated features by reconstructing masking feature values from uncorrupted feature values. Further, the stacking structure and the nonlinearity contribute to mSDA's ability for discovering complex factors behind data. Based on mSDA, our proposed smSDA utilizes semantic dropout noise and sparsity constraints on mapping matrix, in which the efficiency of training can be kept. This extension leads to a stable performance improvement on cyberbullying detection and the detailed analysis has been provided in the following.

We compare the performances of smSDA and $\operatorname{smSDA}_{\mathrm{u}}$, which adopt biased semantic dropout noise and unbiased semantic dropout noise, respectively. The results have shown that $\operatorname{smSDA}_{u}$ performs slightly worse than smSDA. This may be explained by the fact that the unbiased semantic dropout noise cancels the enhancement of bullying features. As shown in Eq. (6.16), the off-diagonal elements in the matrix $\mathbf{x}_{i} \tilde{\mathbf{x}}_{i}^{\mathrm{T}}$ that are used to compute mapping weights are the same, which can not contribute to the reinforcement of bullying features.

Analysis of Semantic Extension As shown in the above section, the semantic extension can boost the performance on classification results for cyberbullying detection. In the following, we discuss the advantages of this extension qualitatively. In our proposed 

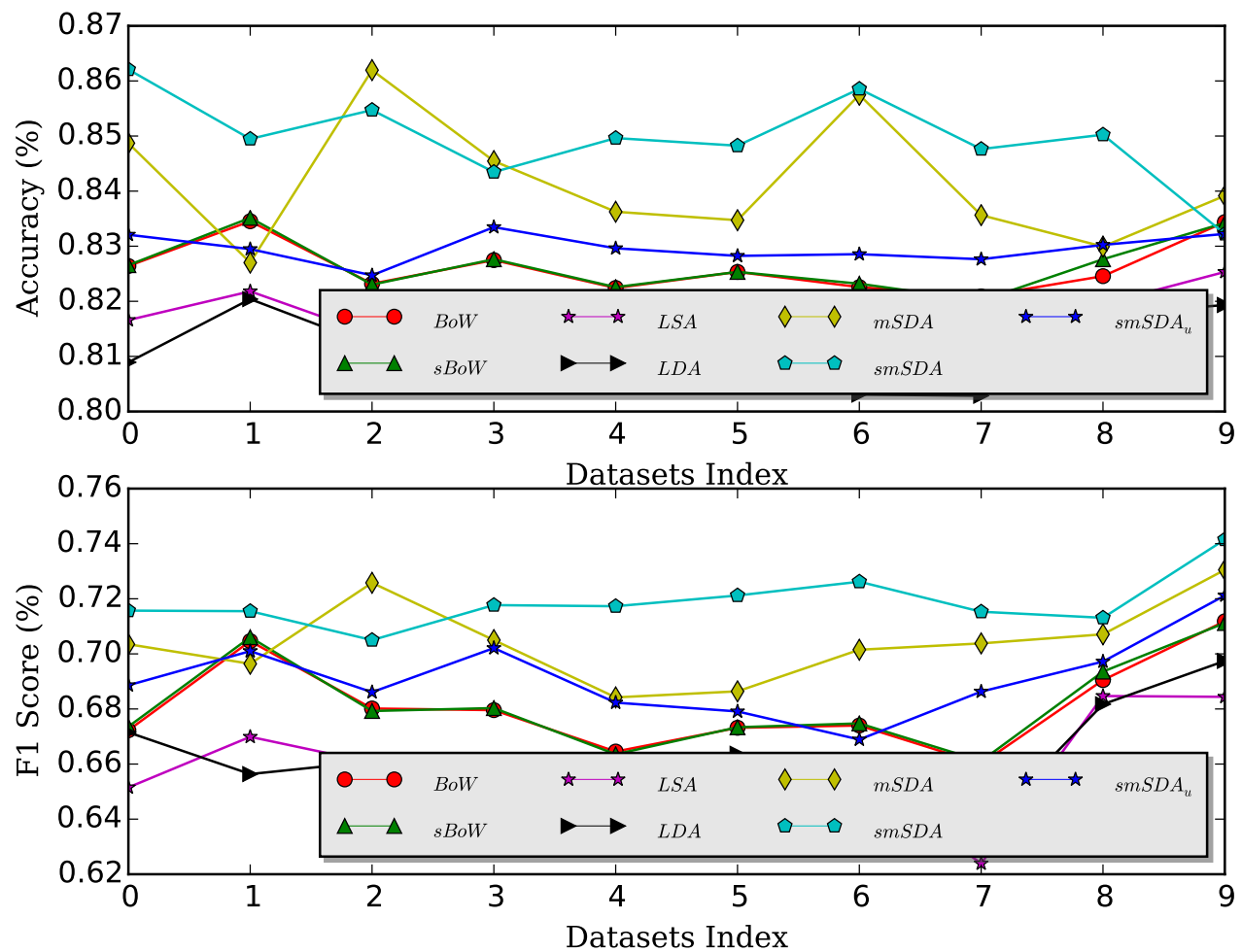

Figure 6.10: Classification Accuracies and F1 Scores of All Compared Methods on Twitter Datasets.

TABle 6.6: F1 Scores (\%) for Compared Methods on Twitter and MySpace Datasets. The Mean Values are Given, respectively. Bold Face Indicates Best Performance.

\begin{tabular}{c||c|c}
\hline Dataset & Twitter & MySpace \\
\hline BWM & 16.1 & 36.4 \\
\hline BoW & 68.1 & 41.2 \\
\hline sBoW & 68.3 & 42.5 \\
\hline LSA & 65.8 & 45.0 \\
\hline LDA & 66.1 & 43.1 \\
\hline mSDA & 70.4 & 76.1 \\
\hline smSDA $_{u}$ & 69.3 & 76.0 \\
\hline smSDA & $\mathbf{7 1 . 9}$ & $\mathbf{7 7 . 6}$ \\
\hline
\end{tabular}



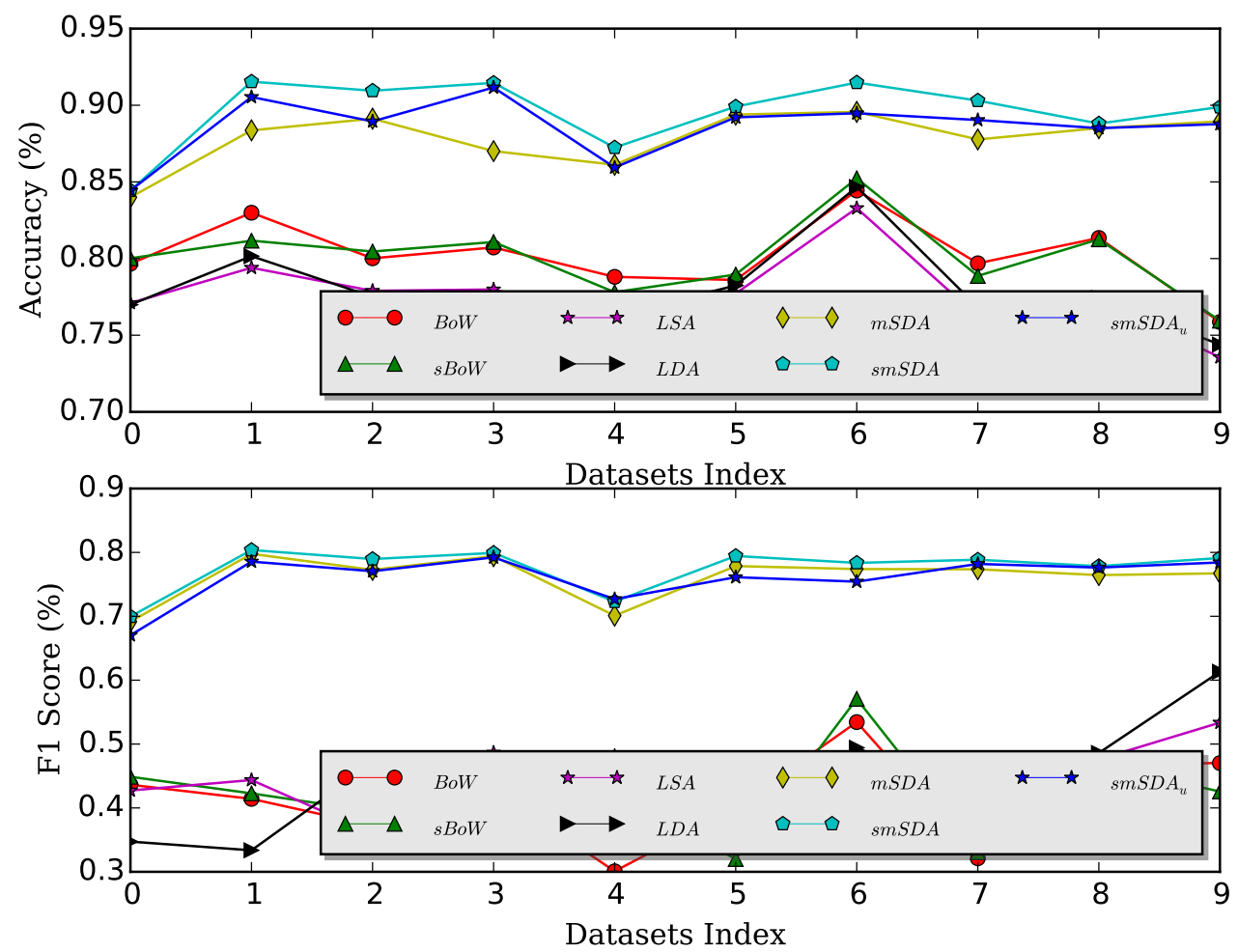

Figure 6.11: Classification Accuracies and F1 Scores of All Compared Methods on MySpace Datasets.

smSDA, because of the semantic dropout noise and sparsity constraints, the learned representation is able to discover the correlation between words containing latent bullying semantics. Table 6.7 shows the reconstruction terms of three example bullying words for mSDA and smSDA, respectively. In this example, one-hot vector is used as input, which represents a document containing one bullying word. Table 6.7 lists the reconstructed terms in decreasing order of their feature values, which represents the strength of their correlations with the input word. The results are obtained using one layer architecture without non-linear activation considering the raw terms directly correspond to each output dimension under such a setting. It is shown that these reconstructed words discovered by smSDA are more correlated to bullying words than those by mSDA. For example, fucking is reconstructed by because, friend, off, gets in mSDA. Except off, the other three words seem to be unreasonable. However, in smSDA, fucking is reconstructed by off, pissed, shit and of. The occurrence of the term of may be due to the frequent misspelling in Internet writing. It is obvious that the correlation discovered by SmSDA is more meaningful. This indicates that smSDA can learn the words' correlations which may be the signs of bullying semantics, and therefore the learned robust features boost the performance on cyberbullying detection. 
TABle 6.7: Term Reconstruction on Twitter datasets. Each Row Shows Specific Bullying Word, along with Top-4 Reconstructed Words (ranked with their frequency values from top to bottom) via mSDA (left column) and smSDA (right column).

\begin{tabular}{c|c|c}
\hline \multirow{2}{*}{ Bullying Words } & \multicolumn{2}{|c}{ Reconstructed Words for } \\
\cline { 2 - 3 } & mSDA & smSDA \\
\hline \multirow{5}{*}{ bitch } & @USER & @USER \\
& shut & HTTPLINK \\
& friend & fuck up \\
& tell & shut \\
\hline \multirow{5}{*}{ fucking } & because & off \\
& friend & pissed \\
& off & shit \\
& gets & of \\
\hline \multirow{5}{*}{ shit } & some & abuse \\
& big & this shit \\
& with & shit lol \\
& lol & big \\
\hline
\end{tabular}

\subsection{Conclusions}

In section 6.2 , we have proposed a novel representation learning method for cyberbullying detection, which is named Embedding-enhanced Bag-of-Words. EBoW concatenates BoW features, latent semantic features and bullying features together. Bullying features are derived based on word embeddings, which can capture the semantic information behind words. When the final representation is learned, a linear SVM is adopted to detect bullying messages. The effectiveness of our proposed model has been experimentally verified through a real-world Twitter corpus.

Section 6.3 addresses the text-based cyberbullying detection problem, where robust and discriminative representations of messages are critical for an effective detection system. By designing semantic dropout noise and enforcing sparsity, we have developed semanticenhanced marginalized denoising autoencoder as a specialized representation learning model for cyberbullying detection. In addition, word embeddings have been used to automatically expand and refine bullying word lists that will be initialized by domain knowledge. The performance of our approaches has been experimentally verified through two cyberbullying corpora from social medias: Twitter and MySpace. 



\section{Chapter 7}

\section{Conclusions}

In this chapter, we summarize the thesis and provide some future research directions.

\subsection{Summary}

Representation learning is the key stone for various natural language processing tasks. As in most scenarios that machine learning models are applied, representation determines the upper bound of performances for natural language processing. Therefore, in this thesis, we have conducted a in-depth study of representation learning for text data.

As reviewed in Chapter 2, the majority of text representation learning models can be categorized into two directions: BoW-based and neural compositional models. BoW-based models normally perform various dimensionality reduction on the document-term occurrence matrix to derive dense and low-dimensional representation of text data. Chapter 3 introduced a Semi-Random Projection framework called SRP, which takes the merit of random feature sampling of RP and employs learning mechanism in the determination of the transformation matrix, with the goal of achieving a good balance between computational efficiency and classification accuracy. Under the SRP framework, we develop one learning scheme based on Liner Discriminative Analysis (LDA) and stack several SRP modules together to learn complex and meaningful representation, which is named stacked SRP (SSRP). The introduction of LDA can enable the learned representation to be discriminative, while random feature subset selection can control the computational complexity. Experiments on two document classification tasks have verified the efficiency and effectiveness of our proposed models. Motivated by the compositionality theory that the semantic meanings of the entity depend on their basic units, neural compositional 
models try to learn complex compositionality upon words via various neural networks including CNN, RNN and so on. The model family is very powerful in theory considering extensive information of texts including word order and syntax can be captured. Chapter 4 focused on the multi-sense word embeddings for neural compositional models. By incorporating probability distribution derived by Latent Dirichlet Allocation, TopCNN and TopLSTMs are able to explore the topic-specific sense of words and further utilize such topic-specific sense jointly with general sense of word embeddings in the semantic compositional process. In addition, several effective architectures for topic embedding learning have been proposed and incorporated into our proposed framework. The experimental results have shown that our proposed models are able to achieve competitive performances compared to the state-of-art models over five sentence classification tasks. The improved performance of TopCNN and TopLSTMs over their original models have also proven the effectiveness of our framework.

In Chapter 5, we explored a novel direction for text representation learning. Via analyzing the BoW models for text representation learning from its adopted exact word matching mechanism, we proposed Fuzzy Bag-of-Words models including FBoW and FBoWC to address issues of sparsity and lack of high-level semantics of BoW representation. Word embeddings are utilized to measure semantic similarity among words and construct fuzzy membership functions of basis terms in BoW space over words in the task-specific corpus. To determine basis terms in BoW space, FBoWC utilizes word clusters, while FBoW directly regards high term-frequencies words as original BoW does. The adoption of word clusters in FBoWC can reduce feature redundancy and improve feature discrimination. Further, the inherent connections between our proposed FBoW frameworks including $\mathrm{FBoW}$ and FBoWC and two typical text representation learning models including BoW-based models and CNN-based neural compositional models were analyzed. The performance of our approaches has been experimentally verified through seven multi-class document categorization tasks.

A brief summary about the description as well as the advantages and disadvantages of all the proposed models is given in Table 7.1. Different from previous chapters that focus on general representation learning models, Chapter 6 proposed two novel representation learning methods for cyberbullying detection via incorporating expert knowledge. In the text-based cyberbullying detection problem, robust and discriminative representations of messages are critical for an effective detection system. The expert knowledge used here is a pre-defined bullying word list. One model named embedding-enhanced BoW concatenates BoW features, latent semantic features and bullying features together. Bullying features are derived from a list of bullying words which is expanded from a pre-defined list via word embeddings. The other model is named semantic-enhanced marginalized 
denoising autoencoder. By designing semantic dropout noise and enforcing sparsity, we have developed a specialized representation learning model for cyberbullying detection. The performance of our proposed models has been experimentally verified through two cyberbullying corpora from social medias: Twitter and MySpace.

The main contributions of this thesis can be summarized as follows:

* A comprehensive review of text representation learning: we provided a comprehensive review of text representation learning based on two major research directions including BoW-based and neural compositional models. Various models in each direction are viewed in an unified framework.

* Three general text representation learning frameworks: we proposed three general frameworks to learn text representation, which are all shown to be effective in various text classification tasks. The first framework SRP is featured by a good balance between computational complexity and discriminative representation learning. Then, the second framework topic-aware deep compositional models enable neural compositional models including CNN and LSTM to capture multisense words. The third framework FBoW is capable of encoding more semantic into the numerical representation by designing a word semantic matching instead of exact word string matchings. Our experiments all show that these three general frameworks can improve the performances especially on text categorization tasks.

* Effective text-based cyberbullying detection: two novel text representation learning models including embedding-enhanced BoW and semantic-enhanced marginalized denoising auto-encoder for cyberbullying detection are proposed. In these two models, domain knowledge, i.e., a pre-defined bullying word list, is utilized. Experiments on two real-life social medial corpus Twitter and MySpace have proven the effectiveness of our models.

\subsection{Future Directions}

At last, we present some potential future research directions as follows:

* Enrich word vector representation: Although in Chapter 4 we introduced topic model to enrich word embeddings, pre-trained word embeddings should be further enhanced for various NLP tasks. In the future, we plan to post-tune existed 
TABLE 7.1: Comparisons of all proposed general text representation models in this thesis.

\begin{tabular}{l||l|l} 
Model & Descr. & Pros/Cons/ \\
\begin{tabular}{l|l} 
SRP \\
Sec. 3.2
\end{tabular} & $\begin{array}{l}\text { text representation } \\
\text { learning based on } \\
\text { semi-random projection }\end{array}$ & $\begin{array}{l}\text { Pro: discriminative; fast; } \\
\text { Con: labels required; word order abandon }\end{array}$ \\
\hline SSRP & $\begin{array}{l}\text { text representation } \\
\text { learning via stacked } \\
\text { structure of semi-random } \\
\text { projections }\end{array}$ & $\begin{array}{l}\text { Pro: discriminative; efficient; deep structure } \\
\text { Con: labels required; word order abandon }\end{array}$ \\
\hline $\begin{array}{l}\text { TopCNN } \\
\text { TopLSTM }\end{array}$ & $\begin{array}{l}\text { supervised sentence } \\
\text { and short-text } \\
\text { classification based on } \\
\text { neural models }\end{array}$ & $\begin{array}{l}\text { Pro: best performance on short-text } \\
\text { classification; capture multi-sense and } \\
\text { order informations behind words; } \\
\text { Con: labels required; careful hyperparameter } \\
\text { selection; time-consuming }\end{array}$ \\
\hline $\begin{array}{l}\text { FBoW } \\
\text { FBoWC }\end{array}$ & $\begin{array}{l}\text { unsupervised document } \\
\text { Sepresentation learning } 5.2\end{array}$ & $\begin{array}{l}\text { Pro: unsupervised; simple; very fast to train; } \\
\text { powerful semantic modeling } \\
\text { Con: not suitable for short-text }\end{array}$ \\
\hline
\end{tabular}

word embeddings. For example, similar idea has been implemented that a taskindependent fine tuning framework based on multi-set word embeddings and lexical semantic resource was proposed in [169].

* Exploit more advanced neural compositional structure: recently, the most popular research direction for text representation learnings is investigating complex neural networks and combinations of neural networks to perform semantic composition. Although such kind of neural compositional models have redefined benchmark performances in various NLP tasks, it still meaningful to investigate whether it is worthy to adopt such a complex model for a insignificant performance improvement compared to conventional n-gram models. In addition, the majority of neural compositional models require high-quality and large-scale training corpus with annotations and careful hyper-parameter selection. Therefore, we plan to develop neural compositional models with few parameters for text representation learning. In addition, unsupervised neural compositional models following an auto-encoder framework can also be considered as another interesting research direction.

* More challenging NLP tasks: In various NLP tasks, text classification is a relatively simple and conventional challenge. More challenging tasks such as question answering should require the models to fully exploit the complexity nature behind 
language and capture its vague semantic. In the future, we are going to investigate the extension of our proposed models and develop novel models to solve this task.

* Deep study of cyberbullying detection: In Chapter 6, it has been shown that discriminative text representation learning model combined with SVM classifier can be an effective cyberbullying detection system. However, it is more challenging and meaningful to predict the occurrence of cyberbullying, since cyberbullying behavior is usually a evolving process. A more systematic approach based on fusion of various information including text, image and interaction on Internet should be proposed to address this issue, which is our future plan. 



\section{Bibliography}

[1] Quoc V Le and Tomas Mikolov. Distributed representations of sentences and documents. In ICML, volume 14, pages 1188-1196, 2014.

[2] Han Zhao, Zhengdong Lu, and Pascal Poupart. Self-adaptive hierarchical sentence model. arXiv preprint arXiv:1504.05070, 2015.

[3] Andrew McAfee, Erik Brynjolfsson, Thomas H Davenport, DJ Patil, and Dominic Barton. Big data. The management revolution. Harvard Bus Rev, 90(10):61-67, 2012 .

[4] Viktor Mayer-Schönberger and Kenneth Cukier. Big data: A revolution that will transform how we live, work, and think. Houghton Mifflin Harcourt, 2013.

[5] Yoshua Bengio, Aaron Courville, and Pascal Vincent. Representation learning: A review and new perspectives. Pattern Analysis and Machine Intelligence, IEEE Transactions on, 35(8):1798-1828, 2013.

[6] Kamal Nigam, Andrew Kachites McCallum, Sebastian Thrun, and Tom Mitchell. Text classification from labeled and unlabeled documents using em. Machine learning, 39(2-3):103-134, 2000.

[7] Rui Zhao and Kezhi Mao. Supervised adaptive-transfer plsa for cross-domain text classification. In Data Mining Workshop (ICDMW), 2014 IEEE International Conference on, pages 259-266. IEEE, 2014.

[8] Bo Pang and Lillian Lee. Opinion mining and sentiment analysis. Foundations and trends in information retrieval, 2(1-2):1-135, 2008.

[9] Michael Steinbach, George Karypis, Vipin Kumar, et al. A comparison of document clustering techniques. In KDD workshop on text mining, volume 400, pages 525526. Boston, 2000.

[10] Karen Sparck Jones. A statistical interpretation of term specificity and its application in retrieval. Journal of documentation, 28(1):11-21, 1972. 
[11] M. Lan, C. L. Tan, J. Su, and Y. Lu. Supervised and traditional term weighting methods for automatic text categorization. IEEE Transactions on Pattern Analysis and Machine Intelligence, 31(4):721-735, April 2009. ISSN 0162-8828. doi: 10. 1109/TPAMI.2008.110.

[12] Gerard Salton and Christopher Buckley. Term-weighting approaches in automatic text retrieval. Information processing $\mathcal{E}$ management, 24(5):513-523, 1988.

[13] Ho Chung Wu, Robert Wing Pong Luk, Kam Fai Wong, and Kui Lam Kwok. Interpreting tf-idf term weights as making relevance decisions. ACM Transactions on Information Systems (TOIS), 26(3):13, 2008.

[14] D. Tang, B. Qin, F. Wei, L. Dong, T. Liu, and M. Zhou. A joint segmentation and classification framework for sentence level sentiment classification. IEEE/ACM Transactions on Audio, Speech, and Language Processing, 23(11):1750-1761, Nov 2015. ISSN 2329-9290. doi: 10.1109/TASLP.2015.2449071.

[15] H. Palangi, L. Deng, Y. Shen, J. Gao, X. He, J. Chen, X. Song, and R. Ward. Deep sentence embedding using long short-term memory networks: Analysis and application to information retrieval. IEEE/ACM Transactions on Audio, Speech, and Language Processing, 24(4):694-707, April 2016. ISSN 2329-9290. doi: 10. 1109/TASLP.2016.2520371.

[16] R. E. Banchs, L. F. DHaro, and H. Li. Adequacy-fluency metrics: Evaluating mt in the continuous space model framework. IEEE/ACM Transactions on Audio, Speech, and Language Processing, 23(3):472-482, March 2015. ISSN 2329-9290. doi: 10.1109/TASLP.2015.2405751.

[17] Yoshua Bengio, Réjean Ducharme, Pascal Vincent, and Christian Janvin. A neural probabilistic language model. The Journal of Machine Learning Research, 3:1137$1155,2003$.

[18] Andriy Mnih and Geoffrey E Hinton. A scalable hierarchical distributed language model. In Advances in neural information processing systems, pages 1081-1088, 2009 .

[19] Tomas Mikolov, Ilya Sutskever, Kai Chen, Greg S Corrado, and Jeff Dean. Distributed representations of words and phrases and their compositionality. In $A d$ vances in neural information processing systems, pages 3111-3119, 2013.

[20] Jeff Mitchell and Mirella Lapata. Vector-based models of semantic composition. In $A C L$, pages 236-244, 2008. 
[21] Katrin Erk and Sebastian Padó. A structured vector space model for word meaning in context. In Proceedings of the Conference on Empirical Methods in Natural Language Processing, pages 897-906. Association for Computational Linguistics, 2008 .

[22] Katrin Erk. Vector space models of word meaning and phrase meaning: A survey. Language and Linguistics Compass, 6(10):635-653, 2012.

[23] Edward Grefenstette and Mehrnoosh Sadrzadeh. Experimental support for a categorical compositional distributional model of meaning. In Proceedings of the Conference on Empirical Methods in Natural Language Processing, pages 1394-1404. Association for Computational Linguistics, 2011.

[24] Richard Socher, Jeffrey Pennington, Eric H Huang, Andrew Y Ng, and Christopher D Manning. Semi-supervised recursive autoencoders for predicting sentiment distributions. In Proceedings of the Conference on Empirical Methods in Natural Language Processing, pages 151-161. Association for Computational Linguistics, 2011.

[25] Richard Socher, Alex Perelygin, Jean Y Wu, Jason Chuang, Christopher D Manning, Andrew Y Ng, and Christopher Potts. Recursive deep models for semantic compositionality over a sentiment treebank. In Proceedings of the conference on empirical methods in natural language processing (EMNLP), volume 1631, page 1642. Citeseer, 2013.

[26] Nal Kalchbrenner and Phil Blunsom. Recurrent continuous translation models. In Proceedings of the conference on empirical methods in natural language processing (EMNLP), pages 1700-1709, 2013.

[27] Kyunghyun Cho, Bart van Merrienboer, Caglar Gulcehre, Fethi Bougares, Holger Schwenk, and Yoshua Bengio. Learning phrase representations using rnn encoderdecoder for statistical machine translation. In Proceedings of the conference on empirical methods in natural language processing (EMNLP), pages 1700-1709, 2014.

[28] Yoon Kim. Convolutional neural networks for sentence classification. In Proceedings of the Conference on Empirical Methods in Natural Language Processing, pages 1746-1751. Association for Computational Linguistics, 2014.

[29] Nal Kalchbrenner, Edward Grefenstette, and Phil Blunsom. A convolutional neural network for modelling sentences. arXiv preprint arXiv:1404.2188, 2014. 
[30] Baotian Hu, Zhengdong Lu, Hang Li, and Qingcai Chen. Convolutional neural network architectures for matching natural language sentences. In Advances in Neural Information Processing Systems, pages 2042-2050, 2014.

[31] Armand Joulin, Edouard Grave, Piotr Bojanowski, and Tomas Mikolov. Bag of tricks for efficient text classification. arXiv preprint arXiv:160\%.01759, 2016.

[32] R. Zhao and K. Mao. Semi-random projection for dimensionality reduction and extreme learning machine in high-dimensional space. IEEE Computational Intelligence Magazine, 10(3):30-41, Aug 2015. ISSN 1556-603X. doi: 10.1109/MCI.2015. 2437316.

[33] R. Zhao and K. Mao. Topic-aware deep compositional models for sentence classification. IEEE/ACM Transactions on Audio, Speech, and Language Processing, 25 (2):248-260, Feb 2017. ISSN 2329-9290. doi: 10.1109/TASLP.2016.2632521.

[34] R. Zhao and K. Mao. Fuzzy bag-of-words model for document representation. IEEE Transactions on Fuzzy Systems, PP(99):1-1, 2017. ISSN 1063-6706. doi: 10.1109/TFUZZ.2017.2690222.

[35] R. Zhao and K. Mao. Cyberbullying detection based on semantic-enhanced marginalized denoising auto-encoder. IEEE Transactions on Affective Computing, PP(99):1-1, 2016. ISSN 1949-3045. doi: 10.1109/TAFFC.2016.2531682.

[36] Rui Zhao, Anna Zhou, and Kezhi Mao. Automatic detection of cyberbullying on social networks based on bullying features. In Proceedings of the 17th International Conference on Distributed Computing and Networking, page 43. ACM, 2016.

[37] Zellig S Harris. Distributional structure. Word, 1954.

[38] Karen Sparck Jones. A statistical interpretation of term specificity and its application in retrieval. Journal of documentation, 28(1):11-21, 1972.

[39] Ian Jolliffe. Principal Component Analysis. Wiley Online Library, 2005.

[40] Gene H Golub and Christian Reinsch. Singular value decomposition and least squares solutions. Numerische Mathematik, 14(5):403-420, 1970.

[41] Bernhard Schölkopf, Alexander Smola, and Klaus-Robert Müller. Kernel principal component analysis. In Artificial Neural NetworksICANN'97, pages 583-588. Springer, 1997. 
[42] Michael E Tipping and Christopher M Bishop. Probabilistic principal component analysis. Journal of the Royal Statistical Society: Series B (Statistical Methodology), 61(3):611-622, 1999.

[43] Shipeng Yu, Kai Yu, Volker Tresp, Hans-Peter Kriegel, and Mingrui Wu. Supervised probabilistic principal component analysis. In Proceedings of the 12th ACM SIGKDD international conference on Knowledge discovery and data mining, pages 464-473. ACM, 2006.

[44] Thomas K Landauer, Peter W Foltz, and Darrell Laham. An introduction to latent semantic analysis. Discourse processes, 25(2-3):259-284, 1998.

[45] Ella Bingham and Heikki Mannila. Random projection in dimensionality reduction: applications to image and text data. In Proc. of 7th ACM SIGKDD International Conference on Knowledge Discovery and Data Mining, pages 245-250. ACM, 2001.

[46] Ping Li, Trevor J Hastie, and Kenneth W Church. Very sparse random projections. In Proc. of 12th ACM SIGKDD International Conference on Knowledge Discovery and Data Mining, pages 287-296. ACM, 2006.

[47] Kevin Beyer, Jonathan Goldstein, Raghu Ramakrishnan, and Uri Shaft. When is nearest neighbor meaningful? In Lecture Notes in Computer Science Volume 1540 - Database Theory - ICDT99, pages 217-235. Springer, 1999.

[48] David E Rumelhart, Geoffrey E Hinton, and Ronald J Williams. Learning internal representations by error propagation. Technical report, DTIC Document, 1985.

[49] Omer Levy, Yoav Goldberg, and Israel Ramat-Gan. Linguistic regularities in sparse and explicit word representations. In CoNLL, pages 171-180, 2014.

[50] Yoshua Bengio, Holger Schwenk, Jean-Sébastien Senécal, Fréderic Morin, and JeanLuc Gauvain. Neural probabilistic language models. In Innovations in Machine Learning, pages 137-186. Springer, 2006.

[51] Frederic Morin and Yoshua Bengio. Hierarchical probabilistic neural network language model. In AISTATS05, pages 246-252, 2005.

[52] Ronan Collobert and Jason Weston. A unified architecture for natural language processing: Deep neural networks with multitask learning. In Proceedings of the 25th international conference on Machine learning, pages 160-167. ACM, 2008.

[53] Tomas Mikolov, Kai Chen, Greg Corrado, and Jeffrey Dean. Efficient estimation of word representations in vector space. arXiv preprint arXiv:1301.3781, 2013. 
[54] Kevin Lund and Curt Burgess. Producing high-dimensional semantic spaces from lexical co-occurrence. Behavior Research Methods, Instruments, \& Computers, 28 (2):203-208, 1996.

[55] Zellig Sabbettai Harris. Mathematical structures of language. 1968.

[56] Marco Baroni and Roberto Zamparelli. Nouns are vectors, adjectives are matrices: Representing adjective-noun constructions in semantic space. In Proceedings of the 2010 Conference on Empirical Methods in Natural Language Processing, pages 1183-1193. Association for Computational Linguistics, 2010.

[57] Emiliano Guevara. A regression model of adjective-noun compositionality in distributional semantics. In Proceedings of the 2010 Workshop on GEometrical Models of Natural Language Semantics, pages 33-37. Association for Computational Linguistics, 2010.

[58] Dimitri Kartsaklis, Mehrnoosh Sadrzadeh, et al. Prior disambiguation of word tensors for constructing sentence vectors. In EMNLP, pages 1590-1601, 2013.

[59] Richard Socher, Brody Huval, Christopher D Manning, and Andrew Y Ng. Semantic compositionality through recursive matrix-vector spaces. In Proceedings of the 2012 Joint Conference on Empirical Methods in Natural Language Processing and Computational Natural Language Learning, pages 1201-1211. Association for Computational Linguistics, 2012.

[60] Xiaodan Zhu, Parinaz Sobhani, and Hongyu Guo. Dag-structured long short-term memory for semantic compositionality. In Proceedings of NAACL-HLT, pages 917926, 2016.

[61] Rui Zhang, Honglak Lee, and Dragomir Radev. Dependency sensitive convolutional neural networks for modeling sentences and documents. In Proceedings of NAACLHLT, pages 1512-1521, 2016.

[62] B Boser Le Cun, John S Denker, D Henderson, Richard E Howard, W Hubbard, and Lawrence D Jackel. Handwritten digit recognition with a back-propagation network. In Advances in neural information processing systems. Citeseer, 1990.

[63] Kevin Jarrett, Koray Kavukcuoglu, Yann Lecun, et al. What is the best multi-stage architecture for object recognition? In 2009 IEEE 12th International Conference on Computer Vision, pages 2146-2153. IEEE, 2009. 
[64] Alex Krizhevsky, Ilya Sutskever, and Geoffrey E Hinton. Imagenet classification with deep convolutional neural networks. In Advances in neural information processing systems, pages 1097-1105, 2012.

[65] Ossama Abdel-Hamid, Abdel-rahman Mohamed, Hui Jiang, and Gerald Penn. Applying convolutional neural networks concepts to hybrid nn-hmm model for speech recognition. In 2012 IEEE international conference on Acoustics, speech and signal processing (ICASSP), pages 4277-4280. IEEE, 2012.

[66] Jürgen Schmidhuber. Deep learning in neural networks: An overview. Neural Networks, 61:85-117, 2015.

[67] Herbert Jaeger. Tutorial on training recurrent neural networks, covering BPPT, RTRL, EKF and the" echo state network" approach. GMD-Forschungszentrum Informationstechnik, 2002.

[68] Ken-ichi Funahashi and Yuichi Nakamura. Approximation of dynamical systems by continuous time recurrent neural networks. Neural networks, 6(6):801-806, 1993.

[69] C Lee Giles, Clifford B Miller, Dong Chen, Hsing-Hen Chen, Guo-Zheng Sun, and Yee-Chun Lee. Learning and extracting finite state automata with second-order recurrent neural networks. Neural Computation, 4(3):393-405, 1992.

[70] Sepp Hochreiter and Jürgen Schmidhuber. Long short-term memory. Neural computation, 9(8):1735-1780, 1997.

[71] Felix A Gers, Jürgen Schmidhuber, and Fred Cummins. Learning to forget: Continual prediction with lstm. Neural computation, 12(10):2451-2471, 2000.

[72] Felix A Gers, Nicol N Schraudolph, and Jürgen Schmidhuber. Learning precise timing with lstm recurrent networks. Journal of machine learning research, 3 (Aug):115-143, 2002.

[73] Junyoung Chung, Caglar Gulcehre, KyungHyun Cho, and Yoshua Bengio. Empirical evaluation of gated recurrent neural networks on sequence modeling. arXiv preprint arXiv:1412.3555, 2014.

[74] Richard Socher. Recursive Deep Learning for Natural Language Processing and Computer Vision. PhD thesis, Citeseer, 2014.

[75] Joseph Reisinger and Raymond J Mooney. Multi-prototype vector-space models of word meaning. In Human Language Technologies: The 2010 Annual Conference of the North American Chapter of the Association for Computational Linguistics, pages 109-117. Association for Computational Linguistics, 2010. 
[76] Eric H Huang, Richard Socher, Christopher D Manning, and Andrew Y Ng. Improving word representations via global context and multiple word prototypes. In Proceedings of the 50th Annual Meeting of the Association for Computational Linguistics: Long Papers-Volume 1, pages 873-882. Association for Computational Linguistics, 2012.

[77] Geoffrey E Hinton. Learning multiple layers of representation. Trends in Cognitive Sciences, 11(10):428-434, 2007.

[78] Yoshua Bengio. Learning deep architectures for AI. Foundations and Trends in Machine Learning, 2(1):1-127, 2009.

[79] Pascal Vincent, Hugo Larochelle, Isabelle Lajoie, Yoshua Bengio, and PierreAntoine Manzagol. Stacked denoising autoencoders: Learning useful representations in a deep network with a local denoising criterion. The Journal of Machine Learning Research, 11:3371-3408, 2010.

[80] Shuicheng Yan, Dong Xu, Benyu Zhang, Hong-Jiang Zhang, Qiang Yang, and Stephen Lin. Graph embedding and extensions: A general framework for dimensionality reduction. IEEE Trans. on Pattern Analysis and Machine Intelligence, 29(1):40-51, 2007.

[81] Geoffrey Hinton, Simon Osindero, and Yee-Whye Teh. A fast learning algorithm for deep belief nets. Neural Computation, 18(7):1527-1554, 2006.

[82] Guang-Bin Huang, Qin-Yu Zhu, and Chee-Kheong Siew. Extreme learning machine: A new learning scheme of feedforward neural networks. In Proc. of 2004 IEEE International Joint Conference on Neural Networks, volume 2, pages 985990. IEEE, 2004.

[83] Guang-Bin Huang, Qin-Yu Zhu, and Chee-Kheong Siew. Extreme learning machine: Theory and applications. Neurocomputing, 70(1):489-501, 2006.

[84] Danny C Sorensen. Implicitly Restarted Arnoldi/Lanczos Methods for Large Scale Eigenvalue Calculations. Springer, 1997.

[85] Hui Zou, Trevor Hastie, and Robert Tibshirani. Sparse principal component analysis. Journal of Computational and Graphical Statistics, 15(2):265-286, 2006.

[86] Xi Chen, Yanjun Qi, Bing Bai, Qihang Lin, and Jaime G Carbonell. Sparse latent semantic analysis. In Proc. of SIAM International Conference on Data Mining, pages 474-485. SIAM, 2011. 
[87] Robert Tibshirani. Regression shrinkage and selection via the lasso. Journal of the Royal Statistical Society. Series B (Methodological), pages 267-288, 1996.

[88] Minmin Chen, Zhixiang Xu, Kilian Weinberger, and Fei Sha. Marginalized denoising autoencoders for domain adaptation. arXiv preprint arXiv:1206.4683, 2012.

[89] Gerard Salton, Anita Wong, and Chung-Shu Yang. A vector space model for automatic indexing. Communications of the ACM, 18(11):613-620, 1975.

[90] K. Bache and M. Lichman. UCI machine learning repository, 2013. URL http: //archive.ics.uci.edu/ml.

[91] Xinxiong Chen, Zhiyuan Liu, and Maosong Sun. A unified model for word sense representation and disambiguation. In Proceedings of the 2014 Conference on Empirical Methods in Natural Language Processing (EMNLP), pages 1025-1035, 2014.

[92] Arvind Neelakantan, Jeevan Shankar, Alexandre Passos, and Andrew McCallum. Efficient non-parametric estimation of multiple embeddings per word in vector space. arXiv preprint arXiv:1504.06654, 2015.

[93] Yang Liu, Zhiyuan Liu, Tat-Seng Chua, and Maosong Sun. Topical word embeddings. In Twenty-Ninth AAAI Conference on Artificial Intelligence, 2015.

[94] Jiwei Li and Dan Jurafsky. Do multi-sense embeddings improve natural language understanding? arXiv preprint arXiv:1506.010\%0, 2015.

[95] Jianpeng Cheng and Dimitri Kartsaklis. Syntax-aware multi-sense word embeddings for deep compositional models of meaning. arXiv preprint arXiv:1508.02354, 2015.

[96] Siva Reddy, Ioannis P Klapaftis, Diana McCarthy, and Suresh Manandhar. Dynamic and static prototype vectors for semantic composition. In IJCNLP, pages 705-713, 2011.

[97] Jianpeng Cheng, Dimitri Kartsaklis, and Edward Grefenstette. Investigating the role of prior disambiguation in deep-learning compositional models of meaning. arXiv preprint arXiv:1411.4116, 2014.

[98] Yann LeCun and Yoshua Bengio. Convolutional networks for images, speech, and time series. The handbook of brain theory and neural networks, 3361(10), 1995.

[99] Hamid Palangi, Li Deng, Yelong Shen, Jianfeng Gao, Xiaodong He, Jianshu Chen, Xinying Song, and Rabab Ward. Deep sentence embedding using long short-term 
memory networks: Analysis and application to information retrieval. IEEE/ACM Transactions on Audio, Speech, and Language Processing, 24(4):694-707, 2016.

[100] Mingbo Ma, Liang Huang, Bowen Zhou, and Bing Xiang. Tree-based convolution for sentence modeling. arXiv preprint arXiv:150\%.01839, 2015.

[101] Meishan Zhang, Yue Zhang, and Duy-Tin Vo. Gated neural networks for targeted sentiment analysis. In Proceedings of the Thirtieth AAAI Conference on Artificial Intelligence, Phoenix, Arizona, USA. Association for the Advancement of Artificial Intelligence, 2016.

[102] David M Blei, Andrew Y Ng, and Michael I Jordan. Latent dirichlet allocation. the Journal of machine Learning research, 3:993-1022, 2003.

[103] Thomas Hofmann. Unsupervised learning by probabilistic latent semantic analysis. Machine learning, 42(1-2):177-196, 2001.

[104] Zhaohui Wu and C Lee Giles. Sense-aaware semantic analysis: A multi-prototype word representation model using wikipedia. In Twenty-Ninth AAAI Conference on Artificial Intelligence, 2015.

[105] Jeffrey Pennington, Richard Socher, and Christopher D Manning. Glove: Global vectors for word representation. Proceedings of the Empiricial Methods in Natural Language Processing (EMNLP 2014), 12:1532-1543, 2014.

[106] Ye Zhang, Stephen Roller, and Byron Wallace. Mgnc-cnn: A simple approach to exploiting multiple word embeddings for sentence classification. arXiv preprint arXiv:1603.00968, 2016.

[107] Wenpeng Yin and Hinrich Schütze. Multichannel variable-size convolution for sentence classification. arXiv preprint arXiv:1603.04513, 2016.

[108] Xiaohui Yan, Jiafeng Guo, Yanyan Lan, and Xueqi Cheng. A biterm topic model for short texts. In Proceedings of the 22nd international conference on World Wide Web, pages 1445-1456. International World Wide Web Conferences Steering Committee, 2013.

[109] Vinod Nair and Geoffrey E Hinton. Rectified linear units improve restricted boltzmann machines. In Proceedings of the 27th International Conference on Machine Learning (ICML-10), pages 807-814, 2010.

[110] Geoffrey E Hinton, Nitish Srivastava, Alex Krizhevsky, Ilya Sutskever, and Ruslan $\mathrm{R}$ Salakhutdinov. Improving neural networks by preventing co-adaptation of feature detectors. arXiv preprint arXiv:120\%.0580, 2012. 
[111] Matthew D Zeiler. Adadelta: An adaptive learning rate method. arXiv preprint $\operatorname{arXiv:1212.5701,2012.}$

[112] Bo Pang and Lillian Lee. Seeing stars: Exploiting class relationships for sentiment categorization with respect to rating scales. In Proceedings of the 43rd Annual Meeting on Association for Computational Linguistics, pages 115-124. Association for Computational Linguistics, 2005.

[113] Bo Pang and Lillian Lee. A sentimen- tal education: Sentiment analysis using subjectivity sum- marization based on minimum cuts. In Proceedings of the $42 n d$ annual meeting on Association for Computational Linguistics, pages 115-124. Association for Computational Linguistics, 2004.

[114] Minqing Hu and Bing Liu. Mining and summarizing customer reviews. In Proceedings of the tenth ACM SIGKDD international conference on Knowledge discovery and data mining, pages 168-177. ACM, 2004.

[115] Janyce Wiebe, Theresa Wilson, and Claire Cardie. Annotating expressions of opinions and emotions in language. Language resources and evaluation, 39(2-3): 165-210, 2005.

[116] Xin Li and Dan Roth. Learning question classifiers. In Proceedings of the 19th international conference on Computational linguistics-Volume 1, pages 1-7. Association for Computational Linguistics, 2002.

[117] Sida Wang and Christopher D Manning. Baselines and bigrams: Simple, good sentiment and topic classification. In Proceedings of the 50th Annual Meeting of the Association for Computational Linguistics: Short Papers-Volume 2, pages 9094. Association for Computational Linguistics, 2012.

[118] Sida Wang and Christopher Manning. Fast dropout training. In Proceedings of the 30th International Conference on Machine Learning (ICML-13), pages 118-126, 2013.

[119] Thomas L Griffiths and Mark Steyvers. Finding scientific topics. Proceedings of the National academy of Sciences of the United States of America, 101(Suppl 1): 5228-5235, 2004.

[120] Derek Greene, Derek O'Callaghan, and Pádraig Cunningham. How many topics? stability analysis for topic models. In Proceedings of the European Conference on Machine Learning and Knowledge Discovery in Databases - Volume 8724, ECML PKDD 2014, pages 498-513, New York, NY, USA, 2014. Springer-Verlag New 
York, Inc. ISBN 978-3-662-44847-2. doi: 10.1007/978-3-662-44848-9_32. URL http://dx.doi.org/10.1007/978-3-662-44848-9_32.

[121] Didier Dubois and Henri Prade. An introduction to fuzzy systems. Clinica Chimica Acta, 270(1):3-29, 1998.

[122] Yan Zhao, James Lam, and Huijun Gao. Fault detection for fuzzy systems with intermittent measurements. Fuzzy Systems, IEEE Transactions on, 17(2):398-410, 2009.

[123] Yanshan Li, Weiming Liu, Qinghua Huang, and Xuelong Li. Fuzzy bag of words for social image description. Multimedia Tools and Applications, pages 1-20, 2014.

[124] Jian-Ping Mei and Lihui Chen. A fuzzy approach for multitype relational data clustering. Fuzzy Systems, IEEE Transactions on, 20(2):358-371, 2012.

[125] N. R. Pal, K. Pal, J. M. Keller, and J. C. Bezdek. A possibilistic fuzzy c-means clustering algorithm. IEEE Transactions on Fuzzy Systems, 13(4):517-530, Aug 2005. ISSN 1063-6706. doi: 10.1109/TFUZZ.2004.840099.

[126] Zhaohong Deng, Fu-Lai Chung, and Shitong Wang. Robust relief-feature weighting, margin maximization, and fuzzy optimization. Fuzzy Systems, IEEE Transactions on, 18(4):726-744, 2010.

[127] William-Chandra Tjhi and Lihui Chen. Dual fuzzy-possibilistic coclustering for categorization of documents. Fuzzy Systems, IEEE Transactions on, 17(3):532$543,2009$.

[128] I-Jen Chiang, Charles Chih-Ho Liu, Yi-Hsin Tsai, and Ajit Kumar. Discovering latent semantics in web documents using fuzzy clustering. Fuzzy Systems, IEEE Transactions on, 23(6):2122-2134, 2015.

[129] Jung-Yi Jiang, Ren-Jia Liou, and Shie-Jue Lee. A fuzzy self-constructing feature clustering algorithm for text classification. Knowledge and Data Engineering, IEEE Transactions on, 23(3):335-349, 2011.

[130] Shie-Jue Lee and Jung-Yi Jiang. Multilabel text categorization based on fuzzy relevance clustering. Fuzzy Systems, IEEE Transactions on, 22(6):1457-1471, 2014.

[131] T. Martin, Y. Shen, and B. Azvine. Incremental evolution of fuzzy grammar fragments to enhance instance matching and text mining. IEEE Transactions on Fuzzy Systems, 16(6):1425-1438, Dec 2008. ISSN 1063-6706. doi: 10.1109/TFUZZ. 2008.925920 . 
[132] S Dumais, G Furnas, T Landauer, S Deerwester, S Deerwester, et al. Latent semantic indexing. In Proceedings of the Text Retrieval Conference, 1995.

[133] Thomas K Landauer, Danielle S McNamara, Simon Dennis, and Walter Kintsch. Handbook of latent semantic analysis. Psychology Press, 2013.

[134] Hongning Wang, Yue Lu, and ChengXiang Zhai. Latent aspect rating analysis without aspect keyword supervision. In Proceedings of the 17th ACM SIGKDD international conference on Knowledge discovery and data mining, pages 618-626. ACM, 2011.

[135] John Blitzer, Mark Dredze, Fernando Pereira, et al. Biographies, bollywood, boomboxes and blenders: Domain adaptation for sentiment classification. In $A C L$, volume 7, pages 440-447, 2007.

[136] Derek Greene and Pádraig Cunningham. Practical solutions to the problem of diagonal dominance in kernel document clustering. In Proc. 23rd International Conference on Machine learning (ICML'06), pages 377-384. ACM Press, 2006.

[137] Matt Kusner, Yu Sun, Nicholas Kolkin, and Kilian Q Weinberger. From word embeddings to document distances. In Proceedings of the 32nd International Conference on Machine Learning (ICML-15), pages 957-966, 2015.

[138] Chih-Chung Chang and Chih-Jen Lin. Libsvm: a library for support vector machines. ACM Transactions on Intelligent Systems and Technology (TIST), 2(3):27, 2011.

[139] Edward A Patrick and FP Fischer. A generalized k-nearest neighbor rule. Information and control, 16(2):128-152, 1970.

[140] Andreas M Kaplan and Michael Haenlein. Users of the world, unite! the challenges and opportunities of social media. Business horizons, 53(1):59-68, 2010.

[141] Robin M Kowalski, Gary W Giumetti, Amber N Schroeder, and Micah R Lattanner. Bullying in the digital age: A critical review and meta-analysis of cyberbullying research among youth. 2014.

[142] M Ybarra. Trends in technology-based sexual and non-sexual aggression over time and linkages to nontechnology aggression. National Summit on Interpersonal Violence and Abuse Across the Lifespan: Forging a Shared Agenda, 2010.

[143] Bridget K Biggs, Jennifer Mize Nelson, and Marilyn L Sampilo. Peer relations in the anxiety-depression link: Test of a mediation model. Anxiety, Stress, 86 Coping, 23(4):431-447, 2010. 
[144] Shane R Jimerson, Susan M Swearer, and Dorothy L Espelage. Handbook of bullying in schools: An international perspective. Routledge/Taylor \& Francis Group, 2010.

[145] Gianluca Gini and Tiziana Pozzoli. Association between bullying and psychosomatic problems: A meta-analysis. Pediatrics, 123(3):1059-1065, 2009.

[146] April Kontostathis, Lynne Edwards, and Amanda Leatherman. Text mining and cybercrime. Text Mining: Applications and Theory. John Wiley $\mathcal{E}$ Sons, Ltd, Chichester, UK, 2010.

[147] Jun-Ming Xu, Kwang-Sung Jun, Xiaojin Zhu, and Amy Bellmore. Learning from bullying traces in social media. In Proceedings of the 2012 conference of the North American chapter of the association for computational linguistics: Human language technologies, pages 656-666. Association for Computational Linguistics, 2012.

[148] Qianjia Huang, Vivek Kumar Singh, and Pradeep Kumar Atrey. Cyber bullying detection using social and textual analysis. In Proceedings of the 3rd International Workshop on Socially-Aware Multimedia, pages 3-6. ACM, 2014.

[149] Brenda L McLaughlin, Anthony A Braga, Carol V Petrie, Mark H Moore, et al. Deadly Lessons:: Understanding Lethal School Violence. National Academies Press, 2002 .

[150] Jaana Juvonen and Elisheva F Gross. Extending the school grounds?bullying experiences in cyberspace. Journal of School health, 78(9):496-505, 2008.

[151] Minne Fekkes, Frans IM Pijpers, A Miranda Fredriks, Ton Vogels, and S Pauline Verloove-Vanhorick. Do bullied children get ill, or do ill children get bullied? a prospective cohort study on the relationship between bullying and health-related symptoms. Pediatrics, 117(5):1568-1574, 2006.

[152] Dawei Yin, Zhenzhen Xue, Liangjie Hong, Brian D Davison, April Kontostathis, and Lynne Edwards. Detection of harassment on web 2.0. Proceedings of the Content Analysis in the WEB, 2:1-7, 2009.

[153] Karthik Dinakar, Roi Reichart, and Henry Lieberman. Modeling the detection of textual cyberbullying. In The Social Mobile Web, 2011.

[154] Vinita Nahar, Xue Li, and Chaoyi Pang. An effective approach for cyberbullying detection. Communications in Information Science and Management Engineering, 2012 . 
[155] Michal Ptaszynski, Fumito Masui, Yasutomo Kimura, Rafal Rzepka, and Kenji Araki. Brute force works best against bullying. In Proceedings of IJCAI 2015 Joint Workshop on Constraints and Preferences for Configuration and Recommendation and Intelligent Techniques for Web Personalization. ACM, 2015.

[156] Maral Dadvar, FMG de Jong, RJF Ordelman, and RB Trieschnigg. Improved cyberbullying detection using gender information. In Proceedings of the 12th Dutch-Belgian Information Retrieval Workshop (DIR2012), Ghent, Belgium, 2012. ACM.

[157] Maral Dadvar, Dolf Trieschnigg, Roeland Ordelman, and Franciska de Jong. Improving cyberbullying detection with user context. In Advances in Information Retrieval, pages 693-696. Springer, 2013.

[158] Gobinda Chowdhury. Introduction to modern information retrieval. Facet publishing, 2010.

[159] Fréderic Godin, Baptist Vandersmissen, Wesley De Neve, and Rik Van de Walle. Named entity recognition for twitter microposts using distributed word representations. In Proceedings of the Workshop on Noisy User-generated Text, pages 146153, Beijing, China, July 2015. Association for Computational Linguistics. URL http://www . aclweb.org/anthology/W15-4322.

[160] Pierre Baldi. Autoencoders, unsupervised learning, and deep architectures. Unsupervised and Transfer Learning Challenges in Machine Learning, Volume 7, page 43, 2012.

[161] Christopher C Paige and Michael A Saunders. Lsqr: An algorithm for sparse linear equations and sparse least squares. ACM Transactions on Mathematical Software (TOMS), 8(1):43-71, 1982.

[162] Michael A Saunders et al. Cholesky-based methods for sparse least squares: The benefits of regularization. Linear and Nonlinear Conjugate Gradient-Related Methods, pages 92-100, 1996.

[163] Jianqing Fan and Runze Li. Variable selection via nonconcave penalized likelihood and its oracle properties. Journal of the American statistical Association, 96(456): 1348-1360, 2001.

[164] C. Vogel. Computational Methods for Inverse Problems. Society for Industrial and Applied Mathematics, 2002. doi: 10.1137/1.9780898717570. URL http://epubs . siam.org/doi/abs/10.1137/1.9780898717570. 
[165] Tran Huy Dat and Cuntai Guan. Feature selection based on fisher ratio and mutual information analyses for robust brain computer interface. In Acoustics, Speech and Signal Processing, 200\%. ICASSP 200\%. IEEE International Conference on, volume 1, pages I-337. IEEE, 2007.

[166] Richard O Duda, Peter E Hart, and David G Stork. Pattern classification. John Wiley \& Sons, 2012.

[167] Junming Sui. Understanding and fighting bullying with machine learning. PhD thesis, THE UNIVERSITY OF WISCONSIN-MADISON, 2015.

[168] Jennifer Bayzick, April Kontostathis, and Lynne Edwards. Detecting the presence of cyberbullying using computer software. In Proceedings of the ACM WebSci'11, pages 1-2, Koblenz, Germany, June 2011. ACM.

[169] X. Yang and K. Mao. Task independent fine tuning for word embedding. IEEE/ACM Transactions on Audio, Speech, and Language Processing, $\operatorname{PP}(99)$ : 1-1, 2016. ISSN 2329-9290. doi: 10.1109/TASLP.2016.2644863. 